\title{
Search for heavy resonances in the dimuon channel with the $\mathrm{D} \varnothing$ detector
}


Cover: Pantaan the Reverberant by Robert Wijtsma, Scheveningen $(\odot) 2009$ 


\title{
Search for heavy resonances in the dimuon channel with the $\mathrm{D} \varnothing$ detector
}

\author{
ACADEMISCH PROEFSCHRIFT
}

\author{
ter verkrijging van de graad van doctor \\ aan de Universiteit van Amsterdam \\ op gezag van de Rector Magnificus \\ prof. dr. D.C. van den Boom \\ ten overstaan van een door het college voor promoties ingestelde \\ commissie, in het openbaar te verdedigen in de Agnietenkapel \\ op dinsdag 24 februari 2009, te 14:00 uur
}

door

Pieter Johannes van den Berg 

Promotor:
prof. dr. S. C: M. Bentvelsen
Co-promotor:
dr. ir. P. J. de Jong

Faculteit der Natuurwetenschappen, Wiskunde en Informatica

ISBN 978-90-9023968-2

This work is part of the research programme of the 'Stichting voor Fundamenteel Onderzoek der Materie (FOM)', which is financially supported by the 'Nederlandse Organisatie voor Wetenschappelijk Onderzoek (NWO)'. 


\section{Contents}

1 Introduction $\quad 1$

2 Theory 3

2.1 The Standard Model . . . . . . . . . . . . . . . . . . . . 3

2.1 .1 Gauge symmetry . . . . . . . . . . . . . . . . . . 3

2.1 .2 The Higgs mechanism $\ldots \ldots \ldots \ldots$

2.1 .3 Renormalization . . . . . . . . . . . . . . . 9

2.1 .4 Quark mixing . . . . . . . . . . . . . . . . . 11

2.1 .5 Phenomenology . . . . . . . . . . . . . . . . . 12

2.2 The $\mathrm{DY}$ interaction . . . . . . . . . . . . . . . . . . . . 13

2.2 .1 DY muon pair production . . . . . . . . . . . 13

2.2 .2 Experimental measurements . . . . . . . . . . . . . 16

2.3 Extensions to the $\mathrm{SM} \ldots \ldots \ldots \ldots \ldots \ldots$

2.3.1 Naturalness . . . . . . . . . . . . . . . . . . . . . . . . . 18

2.3.2 Extended Gauge groups . . . . . . . . . . . . . . . 20

2.3 .3 Generic $\mathrm{U}(1)$ extension $\ldots \ldots \ldots \ldots . \ldots . \ldots 23$

2.3 .4 Little Higgs . . . . . . . . . . . . . . . . . . . . 25

2.3 .5 Extra dimensions . . . . . . . . . . . . . . . 27

3 Experimental Setup $\quad 31$

3.1 Tevatron . . . . . . . . . . . . . . . . . . . . . 31

3.1 .1 Proton Source . . . . . . . . . . . . . . . . 33

3.1 .2 Anti-proton source . . . . . . . . . . . . . . 34

3.2 The $\mathrm{D} \varnothing$ Detector . . . . . . . . . . . . . . . . 36

3.2 .1 Trigger system . . . . . . . . . . . . . . . . . 39

3.2 .2 Luminosity monitors . . . . . . . . . . . . . . . . . . 40

3.2 .3 Central Tracking System . . . . . . . . . . . . 40

3.2 .4 Calorimeter . . . . . . . . . . . . . . . . 44

3.2 .5 Muon system . . . . . . . . . . . . . . . . . . 46 
4 Simulation $\quad 51$

4.1 Monte Carlo samples .................... 52

4.1.1 Background samples .................. 52

4.1 .2 Signal samples ... . . . . . . . . . . . . . 53

4.2 Corrections and uncertainties ................. . . 55

5 Experimental Analysis $\quad 61$

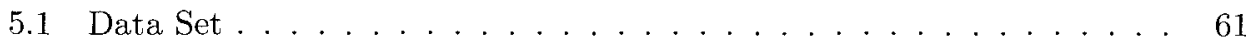

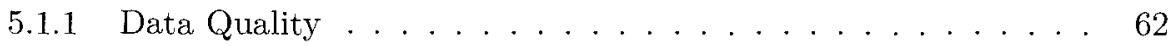

5.1 .2 Luminosity ................... . . 62

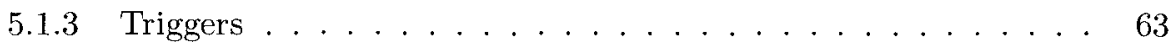

5.2 Muon Reconstruction . . . . . . . . . . . . . . . . . . 63

5.2.1 Central track matching . . . . . . . . . . . . . 65

5.2 .2 Efficiencies ..................... 65

5.2 .3 Track correction .................. 67

5.3 Muon $p_{T}$ smearing . . . . . . . . . . . . . . . . . . 68

$5.3 .1 p_{T}$ resolution uncertainties ................. 70

5.4 Event selection ........................ 70

$5.4 .1 Z$ peak fit ................... 71

5.4 .2 Track quality ....................... 73

5.4 .3 Isolation . . . . . . . . . . . . . . . 74

5.4 Topology ........................ 76

5.4 .5 Cosmic muons ..................... 79

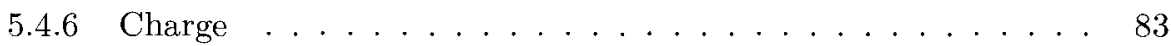

5.4 More than 2 muons . . . . . . . . . . . . . 84

5.4 .8 Final sample . . . . . . . . . . . . . . . 84

5.5 Kinematic fit . . . . . . . . . . . . . . 86

5.5.1 Recoil parameterization .............. 87

5.5.2 Track resolution parameterization ........... . 88

5.5.3 Results of the kinematic fit . . . . . . . . . . . 88

6 Search for new heavy gauge bosons $\quad 95$

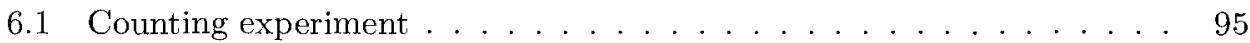

6.1.1 Sensitivity Optimization . . . . . . . . . . . 98

6.2 Bayesian credibility limits . . . . . . . . . . . . . . . 101

6.2.1 Systematic uncertainties on signal efficiency . . . . . . . . 102

6.2 .2 Systematic uncertainty on background . . . . . . . . . 105

6.2.3 Limits on Standard Model-like $Z^{\prime}$ cross section . . . . . . . 106 
6.2.4 Limits for generic $Z^{\prime}$ models from extended gauge groups . . . 108

6.2.5 Limits on Randall-Sundrum model . . . . . . . . . . . 108

6.3 Highest mass event . . . . . . . . . . . . . . . . . . . 113

$\begin{array}{lll}7 \text { Conclusion \& Outlook } & 117\end{array}$

A Results with EW corrections 119

B Triggers $\quad 123$

$\begin{array}{ll}\text { Bibliography } & 125\end{array}$

$\begin{array}{ll}\text { Samenvatting } & 131\end{array}$

$\begin{array}{ll}\text { Acknowledgements } & 133\end{array}$ 


\section{Chapter 1}

\section{Introduction}

Now the smallest Particles of Matter may cohere by the strongest Attractions, and compose bigger Particles of weaker Virtue. There are therefore Agents in Nature able to make Particles of Bodies stick together by very strong Attractions. And it is the business of experimental Philosophy to find them out.

\section{Newton, Opticks}

High-energy particle physics is often described as the quest for a fundamental theory. One can think of one theory as being more "fundamental" than a second theory if the first explains some or all of the predictions of the second in terms of processes at smaller distance scales and higher energies.

Another sense in which a theory can be thought of as being more fundamental than another, is if the first theory "explains" the second; that is, if some arbitrary aspect of the second theory is a special case of a more general principle in the first theory.

The Standard Model of particle physics is the most fundamental experimentally tested theory in either sense. It was developed in its final form in the 1960's and 1970's and continues to successfully predict the outcomes of high-energy physics experiments; the research presented in this thesis offers no exception to this trend (unfortunately).

However, the Standard Model only makes sense as an effective theory; that is, a theory that is not actually fundamental, but which is a low-energy limit of some underlying theory, without being dependent on the details of that underlying theory.

Moreover, one important aspect of the Standard Model so far remains unexplained, namely electroweak symmetry breaking. Phase transitions occur in many places in physics, and can often be explained as a low-energy aspect of some more fundamental process. However, there is no such underlying process for electroweak symmetry breaking within the Standard Model; the introduction of the scalar Higgs field does not, in itself, explain electroweak symmetry breaking, it merely parameterizes it. Therefore, it is perhaps not surprising that the (most obvious) problem with the 
Standard Model, the "gauge hierarchy problem", occurs in relation with the Higgs field.

This problem, the "unnaturalness" of the Standard Model, will be discussed (briefly) in the next chapter. It so happens that the unnaturalness of the Standard Model strongly suggests the presence of new physics at an energy scale that is very close to the energies achieved with the Tevatron collider (and presumably well within the reach of the next collider, the LHC). Therefore, there is a general expectation that new physics is "just around the corner".

Many, many models for new physics exist, and it is not possible to devise an optimal search strategy for all of them at once. Therefore (and possibly to reduce bias), it is preferable to search for new physics in a model-independent way. While the analysis presented in this thesis is certainly not model-independent, it uses the fact that many, often very different models share the prediction of a new mass resonance. Specifically, we search for such resonances in the dimuon channel. Since no new resonance was in fact observed, the various models only serve to parameterize the degree to which nothing was found.

In chapter 2, an outline of the Standard Model is given, and some extensions to the Standard Model are described. Chapter 3 describes the accelerator complex and the $\mathrm{D} \varnothing$ detector.

Chapter 4 describes the simulation of both Standard Model and new physics processes and the detector simulation.

Chapter 5 describes the data sample, the reconstruction algorithms, the event selection and the kinematic fit. A sample of high- $p_{T}$ dimuon events is obtained by applying a number of cuts, and the simulation is tuned to match the efficiencies and track resolution measured on $Z \rightarrow \mu^{+} \mu^{-}$data. The integrated luminosity times the trigger efficiency of the final sample is determined by comparing it to the predicted (and well-tested) $Z$ cross section. The mass resolution is improved by a kinematic fit which uses the transverse energy balance in the event to constrain the muon track $p_{T}$ for each event in the final selection.

In chapter 6, a final cut on the (fitted) invariant mass of the selected events is chosen in such a way that it maximizes the sensitivity to the presence of new physics for specific models and resonance masses. A counting experiment is performed for several values of the mass of a hypothetical new particle. Since no excess over the predicted background is observed, Bayesian credibility limits on the production cross-section times branching ratio of the hypothetical new particles were calculated, as well as limits on the parameters of new physics models. The uncertainties on the theory and detector simulation are taken into account. An event display of the highest reconstructed mass event is shown as well.

Finally, chapter 7 gives a conclusion, some prospects of improving the result and a brief outlook. 


\section{Chapter 2}

\section{Theory}

In this chapter I will describe the central ideas behind the Standard Model and various possible extensions. The Drell-Yan interaction, which is studied in the experimental part, is discussed in more detail.

Some familiarity with the subject matter is assumed. A detailed and thorough standard reference work is [1].

\subsection{The Standard Model}

The Standard Model is the relativistic quantum field theory (QFT) that predicts the strong, weak and electromagnetic interactions of elementary particles.

Relativistic quantum field theory arises from the combination of quantum mechanics, Lorentz invariance and the locality of the fundamental interactions.

The idea that nature is described by a relativistic QFT has many consequences beyond relativity and quantum mechanics, the most important of which may be that different elementary particles have to be exactly indistinguishable.

Another consequence is that the world is symmetric under combined charge conjugation, change of parity (left- or right handedness) and time reversal, called CPT invariance. For one thing, this implies the existence of an anti-particle field for each particle field which transforms under CPT.

The specific relativistic QFT that turns out to describe nature extremely well, called the Standard Model $[2,3,4]$, is subject to other symmetry principles which will be discussed in the following sections. The other idea that is central to the Standard Model is that of renormalization and will be discussed afterwards.

\subsubsection{Gauge symmetry}

Since the gauge symmetries of the Standard Model determine its structure and predictions up to a finite number of coupling constants, it can be argued that they represent 
the most fundamental aspect of the Standard Model. Indeed, the idea of local gauge symmetry has proven to be so powerful in the development of the Standard Model, that in addition most hypotheses beyond the Standard Model rely on symmetry arguments; some of these models will be discussed at the end of this chapter.

Local gauge symmetries are symmetries of the theory under continuous transformations of the fields which form a group and which are different at each point in space or moment in time. Gauge symmetries arise necessarily in quantum field theories that describe the dynamics of particles with integer spin, i.e.. bosons. By a general argument [5], the joint requirements of Lorentz invariance and the ground state energy being bounded from below lead to the conclusion that a Lagrangian for $N_{V}$ spin one fields $A_{\mu}^{1 \ldots N_{V}}$ at high energies and momenta approaches the free vector field Lagrangian

$$
\mathcal{L}_{V}=\sum_{a=1}^{N_{V}}-\frac{1}{4} F_{\mu \nu}^{a} F^{a \mu \nu}, \quad F_{\mu \nu}^{a}=\partial_{\mu} A_{\nu}^{a}-\partial_{\nu} A_{\mu}^{a}
$$

Since the four-component vector fields $A_{\mu}^{a}$ describe the dynamics of a (massless) spin one particle, which is specified by two possible polarizations ${ }^{1}$, there are many field degrees of freedom in this Lagrangian. However, the physical predictions of the theory should not depend on the superfluous degrees of freedom. The unique way to force this to be the case is to require that the theory is invariant under local redefinitions of the fields that can be written as

$$
A_{\mu}^{a}(\mathrm{x}) \rightarrow A_{\mu}^{a}(\mathrm{x})+\partial_{\mu} \phi^{a}(\mathrm{x})+\cdots
$$

for arbitrary scalar fields $\phi^{1 \ldots N_{V}}$ (where one allows for terms not contributing to the kinetic terms in $\mathcal{L}_{V}$ ), since then $\mathcal{L}_{V}$ transforms as

$$
\mathcal{L}_{V} \rightarrow \mathcal{L}_{V}+\text { total derivative }
$$

Each gauge transformation must be an element of a $N_{V}$-dimensional continuous (Lie) group $G$. For quantum electrodynamics (QED), the quantum version of classical electrodynamics, $G$ is the Abelian Lie group $\mathrm{U}(1)$, and the additional terms in equation (2.1) vanish exactly. More generally however, and for the case of the Standard Model, $G$ can be a non-Abelian Lie group and the gauge transformation is

$$
A_{\mu}^{a} \rightarrow A_{\mu}^{a}+\partial_{\mu} \phi^{a}-g f_{a b c} \phi^{b} A_{\mu}^{c}
$$

where $f_{a b c}$ are the structure constants of the group in question and $\mathrm{g}$ is a freely adjustable coupling constant. To make sure the Lagrangian is still gauge invariant, the field strength $F_{\mu \nu}^{a}$ gets an additional contribution $g f_{a b c} A_{\mu}^{a} A_{\nu}^{b}$. This is a self-interaction term, which is absent in the Abelian (electromagnetic) case. Gauge theories with nonAbelian gauge symmetry groups are called Yang-Mills theories.

\footnotetext{
${ }^{1}$ Or it can be seen as the high energy and momentum limit of a massive vector field, which is (in its rest-frame) specified by three possible polarizations.
} 
Formally, the other elementary particle fields $\psi_{i}(\mathrm{x})$ corresponding to the fermions and scalars in the theory can be written as (combinations of) irreducible representations of $G$, so that under an infinitesimal gauge transformation

$$
\psi_{i}(\mathrm{x}) \rightarrow \psi_{i}(\mathrm{x})+\sum_{a=1}^{N_{V}} i \phi^{a}(\mathrm{x}) T^{a} \psi^{i}(\mathrm{x})+\mathcal{O}(\phi)^{2}
$$

where the $T^{a}$ are the $N_{V}$ infinitesimal generators of the gauge group, with $\left[T_{a}, T_{b}\right]=$ $\sum_{c} i g f_{a b c} T_{c}$. It is clear that not every possible term in the Lagrangian will be invariant under these transformations by itself.

If one starts with a Lagrangian $\mathcal{L}_{\text {matter }}$ for these fields and requires that it becomes invariant under gauge transformations, in order to make the kinetic terms gauge invariant one is forced to introduce couplings to the $N_{V}$ vector fields in such a way that each space time derivative in $\mathcal{L}_{\text {matter }}$ gets replaced by a gauge covariant gradient,

$$
\partial_{\mu} \psi_{i}(\mathrm{x}) \rightarrow D_{\mu} \psi_{i}(\mathrm{x}) \equiv\left(\partial_{\mu}+\sum_{a=1}^{N_{V}} i g A_{\mu}^{a}(\mathrm{x}) T^{a}\right) \psi_{i}(\mathrm{x})
$$

In the general case (Abelian and non-Abelian), the vector fields $A_{\mu}^{a}$ must then transform under the gauge group as

$$
A_{\mu}^{a}(\mathrm{x}) \rightarrow A_{\mu}^{a}(\mathrm{x})-\frac{1}{g} \partial_{\mu} \phi^{a}(\mathrm{x})+\sum_{b, c} f_{a b c} \phi^{b}(\mathrm{x}) A_{\mu}^{c}(\mathrm{x})
$$

The vector fields correspond to a special representation (called adjoint) related to the way the group acts on itself,

$$
\left(A_{\mu}\right)_{b c}(\mathrm{x})=A_{\mu}^{a}(\mathrm{x})\left(f_{a}\right)_{b c}
$$

The vector bosons are representations of the gauge group on real vector spaces, whereas the fermions are representations on complex vector spaces. For any complex vector space there is a conjugate vector space related by the usual complex conjugation, and the representations on that space correspond with the anti-particle fields. Lorentz invariance then implies that for every interaction term its conjugate must be included as well, so that the sum is gauge invariant.

In this way, specifying the symmetry group and the representations corresponding to all the fields in the theory determines all the possible interactions in the massless theory.

We can now sketch how this works in the Standard Model. The non-Abelian Lie group that determines the gauge interactions of the Standard Model, up to coupling constants, is

$$
\operatorname{ISpin}(3,1) \times \mathrm{U}(1) \times \mathrm{SU}(2) \times \mathrm{SU}(3)
$$


The ISpin $(3,1)$ factor refers to the symmetries that are geometrical in nature, i.e., that come from the symmetries of space-time, the Lorentz group plus translations. It is therefore called the 'external' symmetry group. The fields transform under this group as vector (spin-1) fields or scalar (spin-0) fields in the case of bosons ${ }^{2}$, or bi-spinors for fermions. In the latter case, there are always two orthogonal representations, which differ only in the sign they have under the parity transformations (chirality) and are called 'right-handed' and 'left-handed'.

The 'internal' gauge group $\mathrm{U}(1) \times \mathrm{SU}(2) \times \mathrm{SU}(3)$ is a compact Lie group, that is to say, it is a direct product of three simple compact Lie groups, which corresponds to rotations in complex vector spaces. The fields in the Lagrangian are written as products of the irreducible representations of these three groups (and of the external group). The $\mathrm{SU}(3)$ part corresponds with the strong interactions, and the $\mathrm{U}(1) \times \mathrm{SU}(2)$ corresponds to the electromagnetic and weak nuclear interactions.

There is a slight twist in that the $\mathrm{U}(1)$ and $\mathrm{SU}(2)$ parts do not refer directly to the electric and weak parts respectively (in the following, $U(1)_{Y}$ denotes the obvious subgroup). Instead, another $\mathrm{U}(1)$ subgroup of $\mathrm{U}(1)_{\mathrm{Y}} \times \mathrm{SU}(2)$ corresponds to the electromagnetic force (we will call this one $\mathrm{U}(1)_{Q}$ ).

The electric charge $Q$ is related to the charge under the product groups ${ }^{3}$ as $Q=$ $I_{3}+Y / 2$, where $I_{3}$ is the charge under the 'third' component of the $\mathrm{SU}(2)$ group, acting by matrix multiplication on the defining representation as

$$
I_{3} \equiv \frac{1}{2} \sigma_{3}=\left(\begin{array}{cc}
1 / 2 & 0 \\
0 & -1 / 2
\end{array}\right)
$$

(called weak isospin for historical reasons) and $Y$ is the charge under the $\mathrm{U}(1)_{\mathrm{Y}}$ factor (called hypercharge). In the same way, the adjoint representation of the 'slanted' subgroup $\mathrm{U}(1)_{Q}$ corresponds to the physical photon, with the adjoint representation of $\mathrm{U}(1)_{\mathrm{Y}}$ and the third component of the adjoint representation of $\mathrm{SU}(2)$ mixing to give the neutral weak vector boson, and the other two components mixing to give the $W^{+}$and $W^{-}$.

The electromagnetic and weak coupling strengths are therefore specified by a common coupling strength and a mixing angle. In this way the electromagnetic and weak forces are said to be unified. (The idea of unification also arises as a motivation for physics beyond the Standard Model. This will be discussed in section 2.3.2).

The left-handed fermions (and right-handed anti-fermions) are combined in one representation, whereas the right-handed fermion fields are in separate representations

\footnotetext{
${ }^{2}$ Graviton fields, which do not enter in the Standard Model but will be discussed later, are described by (spin-2) tensor fields

${ }^{3}$ The irreducible representations are specified by $N$ numbers, where $N$ is the number of generators, which correspond to the charges of the field under the group. For instance, for every integer $q$ there is a unitary irreducible representation $\alpha$ of $\mathrm{U}(1)$ ( - which has a single generator) on $\mathbb{C}$ given by

$$
\alpha \phi=e^{i q \theta} \phi, \quad \phi \epsilon \mathbb{C}, \theta \in \mathbb{R}
$$

One can then define the self-adjoint operator $Q \phi=-\left.i(d \alpha)\right|_{\theta=0} \phi=q \phi$. The observable corresponding to $Q$ is the electric charge. Similar (but not necessarily commuting) quantities exist to specify the irreducible representations of the other groups.
} 


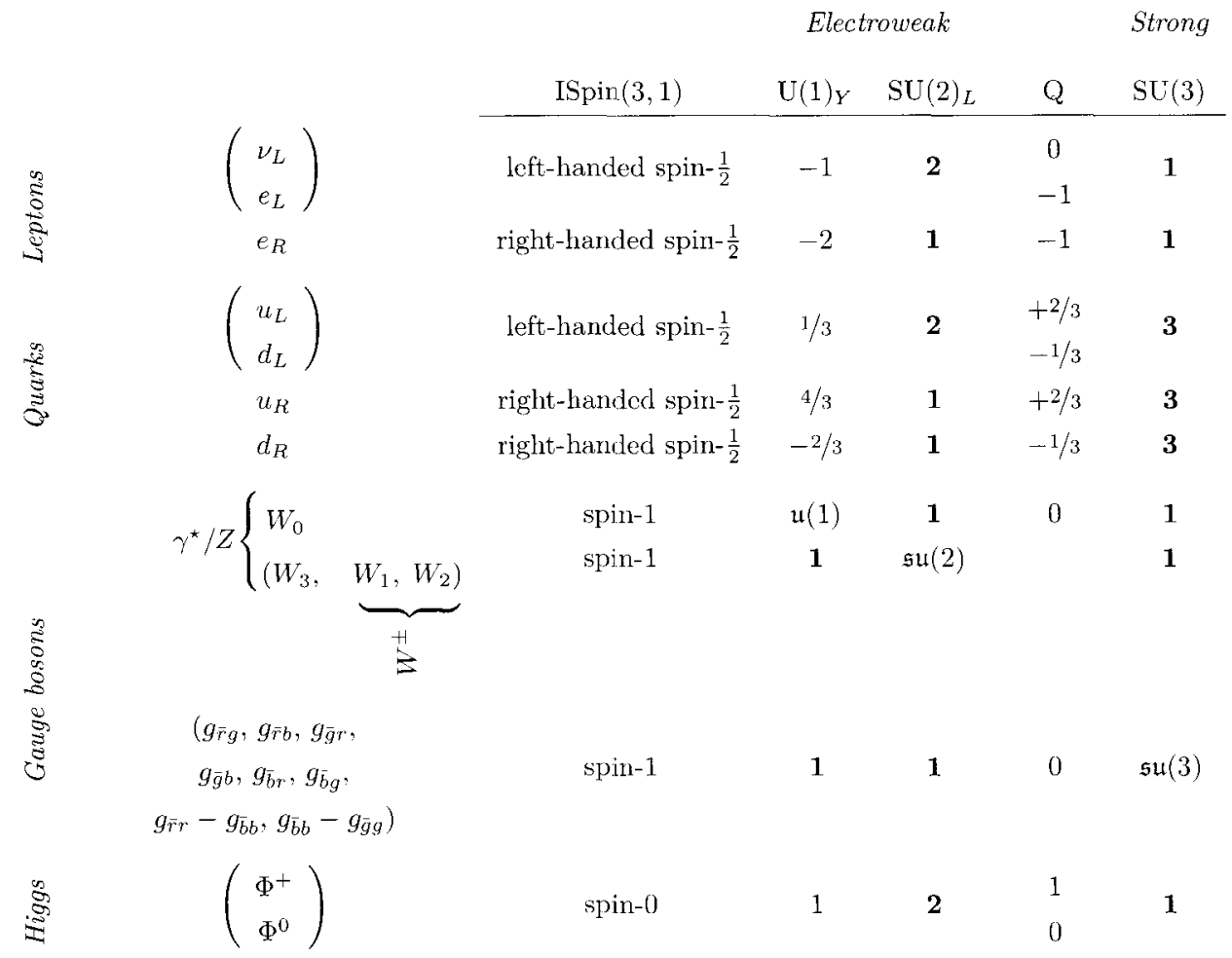

Table 2.1: Fermion fields in one generation, vector boson fields, their representations under the gauge group, as well as their charge under the unbroken $\mathrm{U}(1)_{Q}$. Omitted are the anti-particles of the fermions and Higgs-boson. $\mathfrak{s u}(2)$ etc. indicate the adjoint representations.

that transform trivially under $\mathrm{SU}(2)$. That is to say, an element of $\mathrm{SU}(2)$ transforms a left-handed electron in a left-handed electron-neutrino and a left-handed up-type quark in a left-handed down-type quark, but does nothing on right-handed spinors. This means that the $W^{+}$and $W^{-}$only couple to left-handed fermions, so that the weak force breaks parity maximally.

Table 2.1 lists the force carriers and the fermions in one generation. There exist two more generations of fermions in the same representations, differing only by their masses (cf. table 2.2).

Also omitted is the right-handed neutrino, which was traditionally not included in the Standard Model because a right-handed neutrino with a mass similar to that of the left-handed neutrino has not been observed. Because of the observation of the mixing (see 2.1.4) of neutrinos, it is now thought that right-handed neutrinos may exist after all; however, this will not be discussed here.

There is also a spin-0 field which was not yet discussed. It plays a central role in the Standard Model, generating the masses of all elementary particles in the theory. 


\subsubsection{The Brout-Englert-Higgs mechanism}

The conserved quantity corresponding to the $\mathrm{U}(1)_{Q}$ subgroup is the electric charge, and we know from experiments that, indeed, it is conserved. However, the symmetry under the entire $\mathrm{U}(1) \times \mathrm{SU}(2)$ group does not obviously exist in nature. Since lefthanded electrons and neutrinos are orthogonal components of the same representation of $\mathrm{SU}(2)$, conservation of weak isospin would imply symmetry under the continuous mixing of left-handed electrons with neutrinos (and similarly for their anti-particles). However, in reality neutrinos and electrons are not exchangeable: electrons have electric charge while neutrinos are electrically neutral and their masses differ by, as far as is known today, more than 6 orders of magnitude.

There is another thing about the massless gauge theory which would seem to be in disagreement with observation. We know that some fields are associated with particle states that have a mass: the leptons, the quarks and the weak vector bosons. However, a mass term for the weak vector bosons is not invariant under (2.1).

In addition, the naive way of adding fermion masses to a theory with a non-Abelian symmetry, by simply putting a bilinear scalar term in the Lagrangian for the fermion fields, is made impossible because the two chiral fermion fields have different representations under $\mathrm{SU}(2)$, viz., a right-handed fermion $\mathrm{SU}(2)$ singlet must combine with a left-handed anti-fermion $\mathrm{SU}(2)$ doublet and vice versa to form a scalar.

It can however be shown that if the masses are dynamically generated, they can be accommodated in the theory. The way this happens in the Standard Model is through the same mechanism that breaks the $\mathrm{U}(1) \times \mathrm{SU}(2)$ group down to its $\mathrm{U}(1)_{Q}$ subgroup, and is called the Brout-Englert-Higgs mechanism after a number of its inventors $[3,4,6,7,8,9,10,11,12]$.

The mechanism consists of introducing two new complex spin-0 (i.e.., Lorentz scalar) fields which together form a defining (doublet) representation of $\mathrm{SU}(2)$. It selfinteracts according to a gauge invariant potential

$$
V(\Phi)=\frac{1}{2} \mu^{2} \Phi^{*} \Phi+\frac{1}{4} \lambda\left(\Phi^{*} \Phi\right)^{2}, \quad \mu^{2}<0, \quad \lambda>0
$$

with $\Phi$ as in table 2.1. This results in a non-zero vacuum expectation value

$$
\langle\Phi\rangle=\left(\frac{0}{\sqrt{-\mu^{2} / \lambda}}\right) \text { modulo } \mathrm{U}(1)_{Q}
$$

i.e., it is not locally symmetric under the entire $\mathrm{U}(1)_{\mathrm{Y}} \times \mathrm{SU}(2)$ group, but it has a remaining redundancy in a phase which corresponds to the unbroken $\mathrm{U}(1)_{Q}$ subgroup.

The inclusion of this Higgs field allows for a number of additional invariant terms in the Lagrangian. The leading fermion terms are the trilinear combinations

$$
\psi_{R} \Phi \bar{\psi}_{L}+\text { conjugate }
$$

which are gauge invariant, unlike the bilinear mass terms, because of the doublet representation of $\Phi$; such terms are called Yukawa interactions. Expanding $\Phi=\left(\begin{array}{l}0 \\ v\end{array}\right)+\left(\begin{array}{l}0 \\ H\end{array}\right)$, 
the constant vacuum expectation value $v=246 \mathrm{GeV}$ acts as a mass term, whereas the remaining degrees of freedom $H$ correspond to a new predicted particle, the Higgs particle. The mass of the Higgs particle is an unknown (but constrained) parameter of the theory.

An important difference of the interaction of the Higgs field with Dirac fermions compared to the gauge interactions is thus that the latter preserve chirality, whereas the Higgs force changes a left-handed particle into a right-handed one, and the other way around. Therefore, if a particle exists only in one chirality, it does not couple to the Higgs field and does not acquire a mass. In the traditional Standard Model, this is the case for the neutrinos, which were thought to be massless and hence exist only in a left-handed variety.

The gauge fields which transform non-trivially under the broken part of $\mathrm{U}(1)_{\mathrm{Y}} \times \mathrm{SU}(2)$ interact with $\Phi$ through the gauge covariant derivatives in its kinetic potential; these correspond to the weak vector bosons. Again, the masses are found by considering the terms proportional to powers of $v$ only.

One also finds in this way how the remaining field $H$ couples to the fermions and massive gauge bosons. The Higgs field is a singlet under the $\mathrm{SU}(3)$ gauge symmetry, i.e., it does not interact through the strong force.

This mechanism is usually called 'symmetry breaking', although it is more precise to say that the symmetry is hidden, since the laws of physics still have the same underlying symmetry.

\subsubsection{Renormalization}

In the modern view, the Standard Model is not a 'fundamental' theory in the sense that it could describe all interactions at arbitrarily small distance scales or at arbitrarily large energies. Apart from the fact that this is not testable experimentally, there are several theoretical arguments which preclude this idea.

First, only the part of the Standard Model which describes the strong interaction, $\mathrm{QCD}$, is known to be asymptotically free, i.e., the coupling constant $\alpha_{S}$ approaches zero at small distances. Only asymptotically free theories can be useful to arbitrarily small distances scales, since at a sufficiently large energy scale, for theories that are not asymptotically free all interactions become strong. Secondly, at energies of the order of the Planck scale

$$
M_{\text {Planck }} \approx 1.2 \cdot 10^{19} \mathrm{GeV} / c^{2}
$$

gravity becomes as strong as the other forces and needs to be incorporated in the theory.

For these reasons the Standard Model is thought of as an effective field theory, which describes the physics of a system only up untill some large energy scale (or, equivalently, short distance scale) $\Lambda$. At scale $\Lambda$ the theory is defined by all interactions that are consistent with the symmetry group. Since, in general, an infinite number of such terms can be found, this only defines the theory up to an infinite number of coupling constants, which means such a theory is not in general predictive. 
One can then relate the observables at the scale $\Lambda$ to the observables at some other scale $\mu$ by 'renormalizing' the interactions: starting with the Lagrangian specified at some energy scale $\Lambda$ by the symmetry group $G$ and coupling constants $g_{i}$ with mass dimension $\Delta_{i}$, one can express the coupling constants at the scale $\mu$ as a function of the coupling constants at the scale $\Lambda$, and $\Lambda$ and $\mu$ as

$$
\mu^{-\Delta_{i}} g_{i}(\mu)=F_{i}\left(\Lambda^{-\Delta_{i}} g_{i}(\Lambda), \mu / \Lambda\right)
$$

using the fact that the left-hand side is dimensionless. In general, the coupling strengths at the scale $\mu$ will depend on all coupling strengths at the scale $\Lambda$. However, there exist special theories for which the number of physically distinguishable terms stays finite and fixed. These are called renormalizable theories. It has been shown that the only predictive theories with vector bosons that have this property are gauge theories $[13,14]$.

The non-renormalizable terms in the original Lagrangian are suppressed with powers of $\mu / \Lambda$, so that at a scale $\mu \ll \Lambda$, these terms become vanishingly small and only the renormalizable interactions remain relevant [1]. In fact, this means that at any scale sufficiently smaller than $\Lambda$, only the renormalizable interactions are present in the theory, so that after we calculated the renormalization flow (or 'running') of the various constants in the theory, a finite number of measurements at a particular energy which constrain these constants are enough to make predictions at any other scale sufficiently below $\Lambda$, thereby retaining predictive power in this range.

An additional requirement is that the action of fields with energies $\sim \mu$ satisfies the same gauge symmetries as the original action. In general, a quantum field theory with a bare Lagrangian that obeys certain symmetries, can have those symmetries broken due to quantum effects, so-called anomalies.

In gauge theories with chiral fermions, such as the Standard Model, gauge anomalies arise through certain self-interactions of the vector fields mediated by chiral fermions. In order for the gauge theory to be renormalizable, the gauge anomalies must exactly vanish. Since left-handed and right-handed fermions contribute to the anomalies with opposite sign, this poses a constraint on the number of left- and right-handed fermions in the theory.

In the Standard Model, this leads to the requirement that each generation of elementary particles has the same number of quarks and leptons. Thereby anomaly cancellation ties together the different forces. In grand unified theories the anomaly cancellation follows from the fact that the leptons and quarks are combined in one representation of a larger gauge group; this will be discussed later on.

An intuitive physical picture of renormalization arises in perturbative calculations. Suppose one wants to calculate the measured charge $q$ of an electron at scale $\mu$. The increasing difference between $q(\mu)$ and $q(\Lambda)$ as $\mu$ decreases are due to the increasing number of virtual particle-anti particle pairs in the vacuum being 'seen' over the relevant distance scales which screen or anti-screen the bare charge, i.e. $q(\mu)=$ $q(\Lambda) / \epsilon(\mu)$, where $\epsilon(\mu)$ is the running 'dielectric constant' of the medium that is the sea of virtual particle-anti-particle pairs. The fact that the electromagnetic and weak forces are governed by one coupling constant at sufficiently small distance scales but 
differ at longer distances arises from the fact that the 'vacuum' is polarized differently for them.

The fact that no processes are observed that, in the Standard Model, would be due to non-renormalizable interactions, can therefore be explained by the fundamental energy scale being very large compared to the energies at which we do measurements, so that any physics due to non-renormalizable interactions is highly suppressed. Therefore, in the calculations of observable quantities it suffices to take only the renormalizable interactions into account. The requirement of renormalizability is stringent enough that only a finite number of such interactions are allowed, and in addition gives rise to additional, 'accidental' symmetries, such as lepton and baryon number conservation (which may be broken explicitly in models with larger symmetry groups, discussed below).

Alternatively, one can make the additional assumption that the Lagrangian at the fundamental scale $\Lambda$ for some reason or another only contains the renormalizable terms to begin with. This means that the physics becomes insensitive to $\Lambda$ and that the absence of non-renormalizable interactions is exact. The accidental symmetries implied by renormalizability then also become exact [1].

In calculations, one basically attempts to calculate a quantity at some scale $\mu$ using the 'bare' Lagrangian at scale $\Lambda$, and subsequently calculates counter-terms to account for the difference with the renormalized couplings at scale $\mu$. Since the calculation is done only to some finite accuracy, usually to some finite order in the coupling constants, it can still depend on $\mu$. A conceptual problem arises in that both the calculated quantities and the counter-terms are sometimes infinite, so that regularization is needed to quantify these infinities during the calculation, finding that in the end the result is independent of the regularization procedure that was used. However, it is possible, if not practical or trivial, to calculate the renormalized terms in the perturbative expansion directly, without encountering any infinities [15].

\subsubsection{Quark mixing}

Another feature of the Standard Model is the fact that the fermions are reproduced among three generations, exactly equal except for the masses of the particles. It is not known whether this structure follows from any underlying symmetry principle; it is, for instance, possible to build a consistent Standard Model-like theory with two or four families ${ }^{4}$.

Since the related fermions in the three generations are of the same representation of the gauge group, they are not necessarily independent and can be 'mixed': the mass eigenstate of a (free) particle is then a linear combination of the representations.

Specifically, in the Standard Model the weak representations of the quarks are mixed ${ }^{5}$ through the Higgs mechanism. In effect, the trilinear terms (2.5) are replaced by a complex matrix

\footnotetext{
${ }^{4}$ Although for more than 16 quark flavors the renormalization flow reverses sign and the strong sector is not anymore asymptotically free.

${ }^{5}$ Lepton mixing will not be discussed here.
} 


\begin{tabular}{|ccc|}
\hline $\begin{array}{c}m_{e} \\
0.511 \mathrm{MeV} / c^{2}\end{array}$ & $\begin{array}{c}m_{\mu} \\
105.6 \mathrm{MeV} / c^{2}\end{array}$ & $\begin{array}{c}m_{\tau} \\
1.776 \pm 0.0002 \mathrm{GeV} / c^{2}\end{array}$ \\
\hline$M_{W^{ \pm}}$ & $\Gamma_{W^{ \pm}}$ & $\begin{array}{c}\Gamma_{W^{ \pm} \rightarrow \mu}{ }_{\nu} / \Gamma_{\text {tot }} \\
(10.57 \pm 0.15) \times 10^{-2}\end{array}$ \\
\hline \hline$M_{Z}$ & $2.141 \pm 0.041 \mathrm{GeV} / c^{2}$ & $\Gamma_{Z \rightarrow \mu^{+}{ }^{-} / \Gamma_{\mathrm{tot}}}$ \\
$91.1876 \pm 0.0021 \mathrm{GeV} / c^{2}$ & $2.4952 \pm 0.0023 \mathrm{GeV} / c^{2}$ & $(3.366 \pm 0.007) \times 10^{-2}$ \\
\hline
\end{tabular}

Table 2.2: Lepton masses (rounded off for typographical purposes; the relative error on electron and muon mass is $<10^{-7}$ ) and weak vector boson mass, full width and muonic decay mode branching fraction [16].

$$
Y_{i j}^{d} \bar{q}_{L i} \Phi d_{R j}+Y_{i j}^{d} \bar{q}_{L i} \Phi^{*} u_{R j}+\text { conjugate, } \quad i, j=1 \ldots 3
$$

where the $\mathrm{i}, \mathrm{j}$ indices run over the generations, and $u$ and $d$ indicate the up- and down-type weak singlets. The physical eigenstates are found by diagonalizing the terms proportional to $v$,

$$
M_{\text {diag }}^{u, d}=V_{L}^{u, d} Y^{u, d} V_{R}^{u, d \dagger}(v / \sqrt{2})
$$

Since the weak interactions formally occur in the quark kinetic terms, which are diagonal with respect to the weak eigenstates, the weak interactions are modified to couple quarks of different generations. The unitary matrix $V_{C K M}=V_{L}^{u} V_{L}^{d}$ called the CKM (Cabibbo-Kobayashi-Maskawa) matrix, specifies (together with the gauge coupling strength $g$ ) the coupling strengths of the $W^{ \pm}$. These are extra constants that enter the theory and are not determined through symmetry principles.

\subsubsection{Phenomenology}

We have seen that the symmetries and renormalizability of the Standard Model dictate its structure up to a number of constants. Specifically, these are 19 parameters, which can be counted as the 3 coupling constants, the Higgs mass and self-coupling, the 3 lepton masses and 6 quark masses, and the 4 quark mixing angles, as well as a quantity $\theta_{S}$, related to the the topology of the QCD vacuum. The relative masses and widths of the gauge bosons are predicted by the Standard Model. While it is believed that the masses of baryons are predicted by QCD, they can not be calculated (not counting lattice calculations) and are determined experimentally.

Table 2.2 lists the lepton masses and vector boson properties.

The electromagnetic and weak nuclear forces are weakly interacting, in the sense that cross sections and other observables can be calculated to good accuracy by taking into account only the leading terms in a perturbative expansion in the coupling constants. A specific example of this will be presented in the next section. 
The strong force is described by the unbroken SU(3) theory. At low energies, the strong force is strongly coupled. The result is that free quarks are not seen at lower energies, but only inside hadrons, because the force between two quarks increases with separation rather than decreases; this is called confinement. One important effect of confinement in collider physics is that whenever a light quark or gluon is produced in a hard collision, it will shower (by emitting gluons which decay into quark-anti-quark pairs, etc.) and form a narrow jet of colorless combinations (hadrons and mesons).

Conversely, at high energies, QCD approaches a free field theory. Highly energetic quarks and gluons inside colliding hadrons can scatter off each other like free particles. One way in which this happens in particular at proton-anti-proton colliders is through the Drell-Yan interaction, which is discussed in the next section.

\subsection{The Drell-Yan interaction}

I will now turn to a specific process that occurs in hadron colliders such as the Tevatron (see next chapter), namely, the Drell-Yan production of lepton pairs.[17] By this process, a quark of one (anti-)hadron and an anti-quark of the other hadron annihilate to produce a lepton and its anti-particle in a neutral current with a high invariant dilepton mass.

Such hard scatters are not at all the most prevalent events at a hadron collider. Most elastic collisions produce only particles with a low energy. However, for such processes long range effects are dominant, and the cross section becomes essentially incalculable in perturbation theory. Fortunately, the hard scatters are not only easier to detect and to calculate, but they also are the most likely place to find new physics (that is to say, physics not accommodated in the Standard Model). The hard scattering processes that occur most frequently are $2 \rightarrow 2$ QCD events. The Drell-Yan processes studied here have the additional experimental advantage of being relatively clean events with a clear signature of two high energetic muons, which leads to high detection probability which partly offset the relatively low production cross section. Thus, these type of processes are ideal for discovering new neutral currents.

To lowest order, the Drell-Yan process is described by the resonant production and decay of a neutral vector boson, a $\mathrm{Z}$ or virtual photon. It may receive additional contributions from new neutral vector bosons that are predicted by a slew of theories extending the Standard Model (see next section), the search for which are the subject of this thesis. The Drell-Yan type processes therefore describe both the production of the particles searched for and the most important, irreducible background to this search.

Most of the material in the following section was compiled from [18].

\subsubsection{The Drell-Yan production of muon pairs}

The leading order contribution to the Drell-Yan production of muon pairs is depicted in figure 2.1 . 


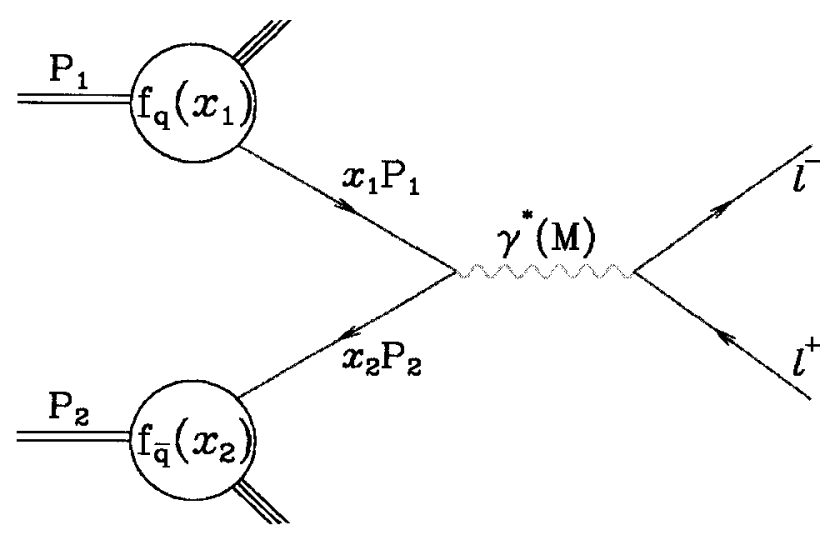

Figure 2.1: Leading order contribution to the Drell-Yan production of lepton pairs.

To a first approximation, at high $M^{2}$ the cross section is given by the lowest order electroweak $q \bar{q} \rightarrow l \bar{l}$ amplitude convoluted with the quark distributions in the proton and anti-protons. This is an immediate consequence of the asymptotic freedom of the strong force: at high energies, the quarks behave as free particles inside the proton and parton-parton interactions can be ignored.

Far below the $Z^{0}$-pole, the hard scattering sub-process cross section is given by

$$
\hat{\sigma}\left(q\left(p_{1}\right) \bar{q}\left(p_{2}\right) \rightarrow \gamma^{*} \rightarrow \ell^{+} \ell^{-}\right)=\frac{4 \pi \alpha^{2}}{3 s} \frac{1}{N_{c}} Q_{q}^{2}
$$

to lowest order in the fine structure constant $\alpha$, where $s=\left(p_{1}+p_{2}\right)^{2}$ is the fourmomentum of the virtual photon, which by momentum conservation equals the dilepton invariant mass squared, $N_{c}$ is the number of colors of the initial state quarks (i.e., three). The factor $1 / N_{c}$ comes from the fact that the initial state quark and anti-quark must be of the same color, since the weak vector bosons are colorless.

The sub-process differential cross section $\frac{d \hat{\sigma}}{d M^{2}}$ for the production of a muon pair with mass $M$ is given by

$$
\frac{d \hat{\sigma}}{d M^{2}}=\frac{\sigma_{0}}{N_{c}} Q_{q}^{2} \delta\left(s-M^{2}\right), \sigma_{0}=\frac{4 \pi \alpha^{2}}{3 M^{2}}
$$

The rapid falling off of the cross section with the invariant dilepton mass is the most striking (if not particularly exciting) phenomenological feature far away from the $Z$-pole. On the $Z$-pole,

$$
\hat{\sigma} \propto \frac{s^{2}}{\left(s-M_{Z}\right)^{2}+\Gamma_{Z}^{2} M_{Z}^{2}}+\mathcal{O}\left(s-M_{Z}\right)
$$

i.e., a Breit-Wigner resonance shape with the narrow width of $\Gamma_{Z}$ (see table 2.2). The peak cross section is 


$$
\hat{\sigma}\left(q \bar{q} \rightarrow Z \rightarrow \mu^{+} \mu^{-}\right)=\frac{\pi}{3} \sqrt{2} G_{F} M_{Z}^{2}\left(V_{q}^{2}+A_{q}^{2}\right)
$$

with

$$
V_{q}=I_{q}^{3}-2 Q_{q} \sin ^{2} \theta_{W}, A_{q}=I_{q}^{3}
$$

(where $I_{q}^{3}$ is the charge of the quark under weak isospin (cf. equation 2.3) and $\theta_{W}$ is the weak mixing angle), neglecting the width of the $\mathrm{Z}$.

To obtain the leading Drell-Yan production cross section, the electroweak amplitude $\hat{\sigma}\left(p_{1,} p_{2}\right)$ must be multiplied by the probability $f_{q}\left(x_{1}\right) \mathrm{d} x_{1}$ of finding a quark with momentum $p_{1}=x_{1} P_{1}$ in one hadron and $f_{\bar{q}}\left(x_{2}\right) \mathrm{d} x_{2}$ of an anti-quark with momentum $p_{2}=x_{2} P_{2}$ in the other hadron, where $P_{1,2}$ are the total momenta of the incoming hadrons. Then we integrate over the momentum fractions and sum over the quark anti-quark pairs.

$$
\sigma_{D Y}=\sum_{q} \int d x_{1} d x_{2} f_{q}\left(x_{1}, \mu_{F}^{2}\right) f_{\tilde{q}}\left(x_{2}, \mu_{F}^{2}\right) \hat{\sigma}_{q \bar{q} \rightarrow \ell^{+} \ell^{-}}\left(x_{1} P_{1}, x_{2} P_{2}, Q^{2} / \mu_{F}^{2}, Q^{2} / \mu_{R}^{2}\right)
$$

$Q$ is the characteristic scale of the hard scattering; this is usually taken to be the mass of the vector boson which mediates the scattering. The dependence on the ultraviolet (i.e. high-energy, short-range) renormalization scale $\mu_{R}$ comes from the fact that $\hat{\sigma}$ is calculated at some fixed order in the coupling parameters.

The factorization scale $\mu_{F}$ is in some sense the infrared (i.e. low energy, long range) analogue of the renormalization scale $\mu_{R}$, and in a perturbative calculation it is related to infrared divergences rather than ultraviolet divergences. Infrared divergences arise if one tries to calculate the contributions involving gluons emitted by the quark or anti-quark that are soft (low energy, hence long range) and collinear (emitted almost along side the quark). Because the partons are interacting strongly at low energies, perturbation theory can not be used to calculate such processes. The divergence of the perturbation series in the soft/collinear limit can be understood to come from the fact that in this limit, a vanishing amount of energy is required to emit an on-shell (since massless) gluon. On-shell propagators imply propagation over long distances, but at distances of the order of the hadron size, $\sim 1 \mathrm{fm}$, non-perturbative confinement and hadronization takes place, which makes the apparent divergences disappear.

It would seem that perturbation theory is therefore useless to calculate processes that are sensitive to infrared physics. However, the situation is slightly better for infrared safe quantities, which are insensitive to long distance effects, and for factorizable quantities, of which the Drell-Yan cross section is an example. The latter have the property that the long distance effects can be absorbed in an overall non-perturbative factor, which can be determined experimentally.

Specifically, one can show that all infrared divergences that arise in the calculation of the subprocess cross section $\hat{\sigma}_{q \bar{q} \rightarrow l^{+} l^{-}}$can be absorbed in the parton distribution functions (PDF) $f_{q}$, which can be determined from inelastic scattering experiments. 
The idea is that the hadron structure as seen by an incoming particle is obtained from an unknown, 'bare' quark distribution, and the probabilities of this quark to interact with the other partons in the hadron, calculated perturbatively. The long-range effects, which can not be calculated perturbatively, are absorbed in the 'bare' quark distribution above some scale $\mu_{F}$. The factorization scale thus determines the scales at which one chooses to separate long-distance effects from short-distance effects. Measuring the hadron structure at $\mu_{F}$ and comparing with the 'renormalized' quark distribution calculated to a particular accuracy gives enough information to obtain the hadron structure at any scale with that accuracy, analogously to the renormalization procedure in the Standard Model at large.

In practice, one often sets the renormalization and factorization scales equal.

In the approximation that the partons have vanishingly small transverse momentum relative to the direction of the parent hadron in the infinite momentum frame, the lepton and anti-lepton are produced exactly back-to-back in the plane transverse to the direction of the incoming proton. However, in reality the partons do have some finite distribution within the proton. Since the distribution of partons inside the proton can not be calculated from first principles, it has to be determined experimentally. A measurement by the CFS collaboration from fixed-target $p N$ collisions at $\sqrt{s}=27.4 \mathrm{GeV}$ measured it to be Gaussian distributed with $\left\langle k_{T}\right\rangle=760 \mathrm{MeV}$ [18]. Therefore the lepton - anti-lepton pair is on average not produced exactly back-to-back and the boson will have a, on average small, transverse boost. ${ }^{6}$

Moreover, the inclusive $q \bar{q} \rightarrow \gamma^{*} / Z+X$ process receives additional contributions of higher order in the coupling parameters, eg. from $2 \rightarrow 2$ processes where the incoming partons scatter strongly, i.e. $q+\bar{q} \rightarrow g+\gamma^{*} / Z$ and $q+g \rightarrow q+\gamma^{*} / Z$. Such contributions enhance the cross section at high transverse vector boson momentum.

K-factors A process such as the Drell-Yan process may be calculated to some finite order in a coupling constant. The orders are denoted by LO (leading order), NLO (next to leading order) and so on. Often, a process may be implemented to some order in a simulation, say LO, but be already known to a higher order, for instance NNLO. The predicted LO cross section from the simulation, with a parton distribution function measured with respect to a fixed order as well, can then be scaled to the cross section at the higher order, neglecting the kinematical details of the difference between the LO and NNLO predictions; the scale factor is called the "K-factor". See section 4.2 for more details.

\subsubsection{Experimental measurements}

The Drell-Yan and $\mathrm{Z}$ boson cross sections have been measured by many experiments on different setups. For a 1993 overview, see [21].

\footnotetext{
${ }^{6}$ Conversely, a measurement of the $Z p_{T}$ distribution constrains the $k_{T}$ distribution; in the analysis of this thesis, the corresponding parameters in the simulation of the Standard Model and new physics prediction are 'tuned' to match the measured boson $p_{T}$ distribution in $Z \rightarrow e^{+} e^{-}$decays. See section 4.2 for details.
} 


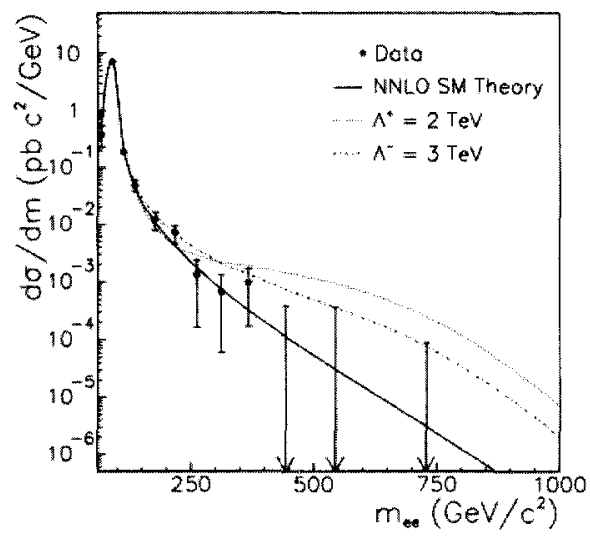

Figure 2.2: $\mathrm{D} \varnothing$ Run I result for the differential inclusive dielectron production cross section [19]. The $68 \%$ uncertainty intervals are shown for the data points. The last three bins, which have no events, show the $84 \%$ C.L. upper limit on the cross section corresponding to the upper end of the error bars in the preceding bins. Also shown is the prediction of the SM at NNLO, and $\mathrm{SM}+$ contact term process (this plot was published in a paper on the limit on leptoquark interactions), at LO corrected with a NNLO $K$ factor.

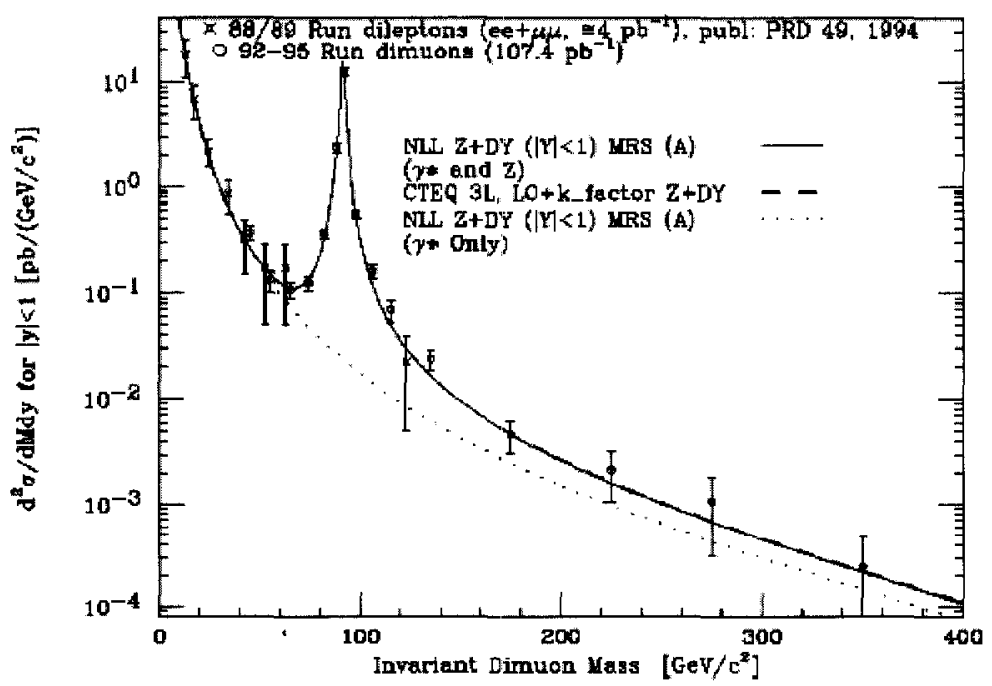

Figure 2.3: CDF Run I Drell-Yan dimuon production cross section extracted from the combined 1992-1993 and 1994-1995 data [20]. The solid line is the NLL (next-to leading logarithmic) QCD prediction using the MRS (A) PDFs. The dashed line is the LO QCD prediction with a $K$ factor to account for higher order effects, calculated with the CTEQ 3L PDFs. The dotted line is the NLL QCD MRS (A) prediction without the contribution from $Z$ exchange. 
At the Tevatron Run I (see next chapter) the first results were obtained on the DrellYan differential cross section at and above the $Z$-resonance in $p \bar{p}$ collisions at $\sqrt{s}=$ $1.8 \mathrm{TeV}$. Figure 2.2 shows the $\mathrm{D} \varnothing$ results in the electron channel, figure 2.3 shows the CDF results.

\subsection{Extensions to the Standard Model}

Even although, as to date, the Standard Model and its calculable derivatives predict correctly the result of many high energy experiments (a significant deviation being the discovery of neutrino mixing), and some to an unprecedented precision, there are physical reasons why we are certain that the Standard Model can not describe physics to arbitrarily small distance scales.

One is that it does not include gravity. Therefore, when the gravitational force between two particles becomes strong, the Standard Model breaks down. This happens at a comfortably small distance scale known as the Planck scale, corresponding to an energy $M_{\text {Planck }} \sim 10^{19} \mathrm{GeV}$.

Another physical reason to suspect the Standard Model can not be the complete is the gauge hierarchy problem, explained below.

Other reasons to look for extensions to the Standard Model are mentioned as well. Simply put, the fact that it describes such a multitude of observations, yet can be constructed from only a few principles, while at the same time being quite rigid in the kind of modifications that are allowed, suggests that the few aspects that do allow for arbitrary or not severely constrained modifications could in an extended theory also follow from underlying symmetries.

\subsubsection{Naturalness}

An effective theory with respect to a scale $\Lambda$ is said to be 'natural' if the values of its free parameters are not sensitive to the physics on distance scales smaller than $\Lambda$. Unfortunately, the Standard Model ceases to be natural for the description of processes with energies larger than around $1 \mathrm{TeV}[22,23]$. The reason is the large difference between the electroweak breaking scale and the Planck scale.

The electroweak breaking scale is of the order of $v \propto m_{H}(\mu)$, with $\mu \ll \Lambda . m_{H}(\mu)$ multiplies a renormalizable term in the effective Lagrangian, and depends in some complicated way on all fields of energies $\mu^{\prime}$ with $\mu<\mu^{\prime}<\Lambda$, but depends on the physics beyond $\Lambda$ only through the values of the couplings and masses $m_{H}(\Lambda), \lambda(\Lambda)$, $g(\Lambda), m_{q}(\Lambda)$, etc., up to effects of order $\Lambda^{-1}$. Similarly, the value of $m_{H}(\Lambda)$ only depends on the physics beyond $\Lambda$.

The leading corrections to $m_{H}$ (or in fact to the mass parameter of any scalar field) are of the form

$$
m_{H}^{2}(\mu)=m_{H}^{2}(\Lambda)+\Lambda^{2}\left(c_{1} \lambda(\Lambda)+\ldots\right)
$$

Dividing (2.7) by $\Lambda^{2}$, we get 


$$
m_{H}^{2}(\mu) / \Lambda^{2}=\mathcal{O}\left(10^{-26}\right)=m_{H}^{2}(\Lambda) / \Lambda^{2}+\left(c_{1} \lambda(\Lambda)+\ldots\right) .
$$

The $c_{i}$ depend on the physics between $\mu$ and $\Lambda$ in some complicated way, whereas the dimensionless term $m_{H}^{2}(\Lambda) / \Lambda^{2}$ depends only on the physics beyond the scale $\Lambda$. The requirement that the first and the second term cancel to 26 orders of magnitude therefore amounts to a "conspiracy" between the low and high energy physics. This is deemed "unnatural", because we expect the physics at large distances to follow from the physics at small distances, and not the other way around.

This does not mean that any small parameter is unnatural. If there exists some approximate symmetry, forming a group with all other symmetries in the theory, that becomes exact (at the quantum level) if some parameter $\alpha$ approaches zero, all corrections to $\alpha$ must necessarily be proportional to $\alpha$ itself. In that case no cancellations are necessary (because there really is a "conspiracy").

One could hope that there is such an approximate symmetry protecting $m_{H}$. However, the only symmetry consistent with gauge symmetry is a symmetry under transformations

$$
\Phi(\mathbf{x}) \rightarrow \Phi(\mathbf{x})+\Omega(\mathbf{x})
$$

which would imply that the Higgs field is an unphysical degree of freedom, since a gauge can be chosen in which it vanished at each point in space-time.

Therefore, the Standard Model with $m_{H} \ll \Lambda$ is 'unnatural' and one speaks here of the gauge hierarchy problem. The unnaturalness of the Standard Model is therefore a hint that it will be modified at a scale that is much lower than $M_{\text {Planck }}$, not more than an order of magnitude larger than $v$ [23].

Experimental constraints favor a light Higgs mass, which leads to a relatively light scale of new physics. However, electroweak precision measurements match the Standard Model very well and therefore seem to favor a large scale for any new physics that modifies the relevant low-energy observables. Therefore, the new physics has to be weakly coupled and affect the low-energy observables only through radiative corrections.

An attractive way of modifying the Standard Model is by embedding the gauge group in one simple gauge group. However, in general these models introduce other gauge hierarchies. Such extensions are detailed in the next section.

Another theory, in which the gauge hierarchy arises naturally, is the Randall-Sundrum model, on which limits are presented in this thesis, and which is discussed at the end of this chapter. This model postulates an extra space dimension, where the apparent relative weakness of gravity is caused by the fact that it propagates in a larger space than the gauge fields.

In theories with super-symmetry an extra (hidden) symmetry between fermions and bosons forces the higher order contributions to $m_{H}$ to vanish exactly (up to supersymmetry breaking terms), so that $m_{H}(\mu)=m_{H}(\Lambda)$. While super-symmetry is the most popular extension of the Standard Model, it will not be discussed further because it is not directly relevant for the searches presented here. 


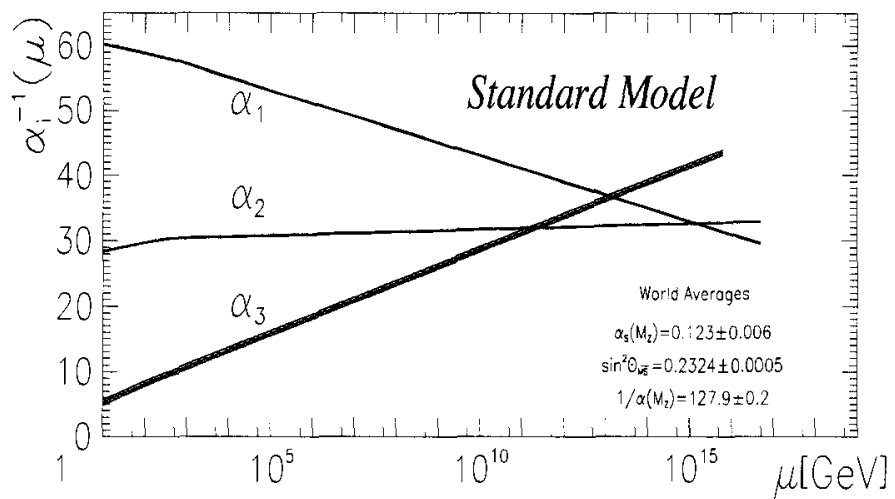

Figure 2.4: Evolution of the inverse of the three coupling constants in the Standard Model, where $\alpha_{1,2,3}$ are the electromagnetic, weak and strong couplings respectively. The width of the lines represent the error in the coupling constants. The evolution of the couplings was calculated to second order [24].

In 'little Higgs' theories, also described in more detail later, the Higgs particle arises as a pseudo Nambu-Goldstone boson associated with a spontaneously broken global symmetry, so that its mass is protected and the hierarchy is no longer unnatural.

\subsubsection{Extended Gauge groups}

\section{Motivation}

The motivation for an extended gauge group is twofold.

First, if one follows the renormalization flow towards higher energies there is an energy $\Lambda_{\mathrm{GUT}} \sim 10^{15} \mathrm{GeV}$ at which all three effective coupling constants are nearly (but not quite) equal; see figure 2.4. That they do so rather than cross at entirely different scales, or not at all, can be seen as a hint that at $\Lambda_{\mathrm{GUT}}$ the three forces combine into one force with one coupling strength, corresponding to one gauge group $\mathrm{G}$ which is broken down to $\mathrm{U}(1) \times \mathrm{SU}(2) \times \mathrm{SU}(3)$ below $\Lambda_{\mathrm{GUT}}$, analogously to the way QED fits into the electroweak part of the Standard Model. Gravity becomes strongly coupled at the Planck energy, which is 'only' $2-3$ orders of magnitude larger than $\Lambda_{\mathrm{GUT}}$.

Secondly, the way in which $\mathrm{U}(1) \times \mathrm{SU}(2) \times \mathrm{SU}(3)$ can be embedded in larger simple groups provides additional relations between the different representations under the subgroups and can therefore be said to 'explain' these relations, which appear to be 'coincidental' in the Standard Model. I will sketch how this works.

$\mathrm{U}(1) \times \mathrm{SU}(2) \times \mathrm{SU}(3)$ has a so-called 'normal' subgroup that acts trivially on all fields in the Standard Model. It is isomorphic to $\mathbb{Z} / 6$ (the integers modulo 6 ), so that the smallest symmetry group of the Standard Model is in fact $\mathrm{U}(1) \times \mathrm{SU}(2) \times \mathrm{SU}(3) /(\mathbb{Z} / 6)$. This group has the nice property that it is in fact isomorphic to a subgroup of SU(5) which has a representation as matrices that can be written as 


$$
\left(\begin{array}{ll}
g & 0 \\
0 & h
\end{array}\right)
$$

where $g$ is $3 \times 3$ and $\mathrm{h}$ is $2 \times 2$ (see, for instance, [25]). One might call it $\mathrm{S}(\mathrm{U}(2) \times \mathrm{U}(3)$ ). This formulation suggests that the 'true' gauge group of the Standard Model is SU(5). In fact, it was shown by Georgi and Glashow [2] that $\mathrm{SU}(5)$ is the smallest suitable Lie group in which the Standard Model group fits. The only other group that allows complex representations, which are needed for chiral fermions, is $\mathrm{SU}(3) \times \mathrm{SU}(3)$, but this group cannot accommodate representations with relatively 'fractional' charges, needed for the quarks to have multiples of $1 / 3$ of the electron charge.

\section{The Georgi-Glashow SU(5) model}

While the simplest SU(5) model, the Georgi-Glashow model, is ruled out by experiment, it serves as a good example of a Grand Unified Theory (GUT). One can write down an $\mathrm{SU}(5)$ gauge theory in the manner indicated in the previous section. One 5 and one 10 dimensional chiral representation together with their duals suffice for all fermions and their anti-particles in one generation:

$$
\left(d^{r}, d^{g}, d^{b}, e^{+}, \bar{\nu}\right)_{R}, \quad\left(\begin{array}{ccccc}
0 & \bar{u}^{r} & \bar{u}^{g} & u^{r} & d^{r} \\
-\bar{u}^{r} & 0 & \bar{u}^{b} & u^{g} & d^{g} \\
-\bar{u}^{g} & -\bar{u}^{b} & 0 & u^{b} & d^{b} \\
-u^{r} & -u^{g} & -u^{b} & 0 & e^{+} \\
-d^{r} & -d^{g} & -d^{b} & -e^{+} & 0
\end{array}\right)_{L}
$$

Nice features of this theory are that it automatically gives an explanation for the fact that the quarks have an electric charge of $1 / 3$ relative to the leptons, since this follows directly from the fact that the trace over the 5-dimensional representation must be zero. The theory is also anomaly-free, and does not need new fermions.

In addition to the Higgs field, one has to introduce extra scalar fields analogous to the Higgs field which acquire a vacuum expectation value that breaks $\mathrm{SU}(5) \rightarrow$ $\mathrm{U}(1) \times \mathrm{SU}(2) \times \mathrm{SU}(3) /(\mathbb{Z} / 6)$ at some energy scale $\Lambda_{G U T}$. The new gauge bosons, which correspond to the broken part of SC(5), thereby acquire a mass of the order of $\Lambda_{G U T}$.

There will be $5^{2}-1$ gauge bosons, $\left(3^{2}-1\right)+\left(2^{2}-1\right)+(1)$ of which are identified with the 8 gluons, 3 weak bosons and the photon respectively. The other half are new predicted gauge bosons which mediate diquark and lepton-quark interactions because they transform both under the adjoints of $\mathrm{SU}(2) \times \mathrm{U}(1)$ and $\mathrm{SU}(3)$.

These kind of processes lead to baryon $(B)$ and lepton $(L)$ number violation, which were 'accidental' symmetries in the Standard Model, in the sense that they are broken only by non-renormalizable interactions. A consequence of this is that the $\mathrm{SU}(5)$ model predicts a finite proton lifetime of [26] 


$$
\tau_{p} \sim \frac{1}{\alpha_{(5)}^{2}} \frac{m_{X}^{4}}{m_{p}^{5}}
$$

where $\alpha_{(5)}$ is the $\mathrm{SU}(5)$ coupling constant and $m_{X}$ is the mass of the gauge bosons of the broken part, proportional to the SU(5) breaking scale. The experimental limit $\tau_{p} \gtrsim 10^{33}$ years [16] gives $m_{X} \gtrsim 10^{20} \mathrm{GeV}$. However, for reasons outlined above, the GUT scale should be smaller than the Planck scale $M_{\text {Planck }} \approx 1.2 \cdot 10^{19} \mathrm{GeV}$.

The theory also predicts a value of the ratio of the electromagnetic and weak nuclear force. This is found by requiring that at or above the unification scale $\Lambda_{G U T}$, the theory is described by one coupling, which fixes the relative strength. The ratio at a lower energy is then found by renormalizing the GUT values. It turns out that the predicted value is ruled out by(but quite close to) the measured one $\left(\sin \theta_{W}^{2} \approx 0.21\right.$ vs. the measured value of $0.23120(5))$.

\section{Larger groups}

It is clear that the Georgi-Glashow model is therefore experimentally excluded. In addition, the theory still suffers from an explicit large hierarchy $m_{X} \gtrsim 10^{12} m_{W}$, which means that this theory is unnatural, like the Standard Model itself. Moreover, it does not include a right-handed neutrino, the existence of which is indicated by the observation of neutrino mixing. Arguably, another deficit is that two representations are needed to fit all the particles, rather than one.

For these reasons, when conceiving of GUTs one is forced to consider larger gauge groups. These larger groups necessarily introduce representations with new fermions. Moreover, any such further enlarged gauge symmetry introduces additional neutral massive gauge bosons in addition to the $\mathrm{Z}$.

For instance, the next larger interesting group is $\mathrm{SO}(10)$. There, all fermions of a generation plus a right-handed neutrino fit in one irreducible 16 dimensional chiral spinor representation of $\mathrm{SO}(10)$. Because of the right-handed neutrino, neutrino masses can be accommodated in this theory.

The new neutral gauge boson comes from the extra Abelian subgroup which fits into $\mathrm{SO}(10)$ besides the Standard Model group. This can be seen through the maximal subgroup decomposition $\mathrm{SO}(10) \supset \mathrm{SU}(5) \times \mathrm{U}(1)$. The new $\mathrm{U}(1)$ symmetry corresponds to the conservation of baryon number minus lepton number, $B-L$.

Even larger groups can be considered, which introduce more new gauge bosons and new 'exotic' fermions. For instance, super-string theories favor the exceptional group $\mathrm{E}_{6}$ which has a maximal decomposition $\mathrm{E}_{6} \supset \mathrm{SO}(10) \times \mathrm{U}(1)$. The breaking pattern

$\mathrm{E}_{6} \rightarrow \mathrm{SO}(10) \times \mathrm{U}(1)_{\psi} \rightarrow \mathrm{SU}(5) \times \mathrm{U}(1)_{\chi} \times \mathrm{U}(1)_{\psi} \rightarrow \mathrm{SU}(3) \times \mathrm{SU}(2) \times \mathrm{U}(1)_{Y} \times \mathrm{U}(1)_{\chi} \times \mathrm{U}(1)_{\psi}$

gives rise to a physical $Z^{\prime}$ which is a mixture of the two new $\mathrm{U}(1)$ gauge bosons,

$$
Z^{\prime}(\theta)=Z_{\psi} \cos \theta^{\prime}+Z_{\chi} \sin \theta^{\prime}
$$


Commonly considered choices for the mixing angle $\theta^{\prime}$ are $0, \arctan \sqrt{3 / 5}$ and $\pi / 2$, called $Z_{\psi}, Z_{\eta}$ and $Z_{\chi}$ respectively. These models do require some extra fermions and need fine tuning to avoid the electroweak precision bounds.

In the research presented in this thesis, only the phenomenology of the extra neutral gauge bosons is studied, not the effects of possible extra fermions.

\subsubsection{Generic $\mathrm{U}(1)$ extension}

Motivated by the above, one can also look at generic extensions of the Standard Model group with a new neutral spin-1 (gauge) boson. The new particle is generally called $Z^{\prime}$. Free parameters ${ }^{7}$ are the $Z^{\prime}$ mass $M_{Z^{\prime}}$, the angles which determine the mixing between the $Z^{\prime}$ and the Standard Model $Z$, and the gauge couplings of the extended gauge group. The generic $Z^{\prime}$ Lagrangian can then be written as [16]

$$
\begin{aligned}
\mathcal{L}_{Z^{\prime}}=-\frac{1}{4} F_{\mu \nu}^{\prime} F^{\mu \nu}+M_{Z^{\prime}}^{2} Z^{\prime 2} & -\frac{e}{2 c_{W} s_{W}} \sum_{i} \bar{\psi}_{i} \gamma^{\mu}\left(f_{V}^{i}-f_{A}^{i} \gamma^{5}\right) \psi_{i} Z_{\mu}^{\prime} \\
& +\delta M_{Z Z^{\prime}}^{2} Z_{\mu}^{\prime} Z^{\mu}-\frac{1}{2} \sin \chi F_{\mu \nu}^{\prime} F^{\mu \nu}
\end{aligned}
$$

where $F^{(\prime)}, M_{Z^{(\prime)}}$ are the $Z^{(\prime)}$ field strength and mass, $c_{W}$ and $s_{W}$ are the cosine and sine of the weak mixing angle. The mass terms are assumed to be generated by a symmetry breaking mechanism, as in the Standard Model, with the term proportional to $\delta M_{Z Z^{\prime}}^{2}$ due to scalar field condensates that are charged under both groups.

The last ('kinetic mixing') term is only gauge invariant for the case that the extended gauge group is Abelian, since for non-Abelian extensions $F_{\mu \nu}^{\prime}$ is not gauge invariant by itself. The effect of this term is to change the $Z-Z^{\prime}$ mixing and the $\mathrm{U}(1)^{\prime}$ charges of all fields [27]. However, in most analyses, as in the searches presented here, $\chi$ is taken to be zero, even for Abelian extensions.

The mixing angle between the $Z$ and $Z^{\prime}, \theta_{Z-Z^{\prime}}$, is related to the entries of the mass matrix as

$$
\tan 2 \theta_{Z-Z^{\prime}}=\frac{2 \delta M_{Z Z^{\prime}}^{2}}{M_{Z^{\prime}}^{2}-M_{Z}^{2}}+\mathcal{O}(\chi)
$$

The $Z-Z^{\prime}$ mixing has the effect of modifying Z-pole observables. Low-energy measurements and electroweak precision measurements (discussed below) severely constrain mixing to be very small.

The couplings to fermions are described by 15 new parameters in the theory corresponding with the charges $z_{f}$ under $\mathrm{U}(1)^{\prime}$, however, the charges must obey certain relations so that the $\mathrm{U}(1)^{\prime}$ anomalies cancel (or in the case there are extra exotic

\footnotetext{
${ }^{7}$ In theories with additional symmetries these parameters can be constrained, for instance in super-symmetric GUTs, super-gravity or theories motivated by string theory, but these will not be discussed here.
} 
fermions that the $\mathrm{U}(1)^{\prime}$ anomalies and the anomalies of the exotic fermions under the SM group cancel). Under mild assumptions it can then be shown that far above the $Z$ mass the cross section for $Z^{\prime}$ production in $p \bar{p}$ collisions with a decay to two muons is given by $[28]$

$$
\sigma\left(p \bar{p} \rightarrow Z^{\prime} X \rightarrow \mu^{+} \mu^{-} X\right)=\frac{\pi}{48 s}\left[c_{u} w_{u}\left(s, M_{Z^{\prime}}^{2}\right)+c_{d} w_{d}\left(s, M_{Z^{\prime}}^{2}\right)\right]
$$

where $w_{u, d}$ are the parts of the hadronic structure function which do not depend on any coupling, and

$$
c_{u, d}=g_{z}^{2}\left(z_{q}^{2}+z_{u, d}^{2}\right) \times \operatorname{Br}\left(Z^{\prime} \rightarrow \mu^{+} \mu^{-}\right)
$$

contain all dependence on the $Z^{\prime}$ couplings to fermions, with $g_{z}$ being the $\mathrm{U}(1)^{\prime}$ gauge coupling and $z_{f}$ the fermion charges.

Many anomaly-free solutions for the $z_{f}$ exist. In [28], four classes of one-parameter solutions are found where the charge under the $\mathrm{U}(1)^{\prime}$ is generation-independent.

One solution is the $\mathrm{U}(1)_{B-L}$ model, where the fermion charges are proportional to $B-L$, baryon number minus lepton number. This symmetry occurs in the left-right symmetric models, which requires a right-handed neutrino for each generation, whose existence is indicated by neutrino mixing. If extra fermions are allowed, a larger class of one-parameter solutions exists for which the fermion charges are proportional to $B-x L$ (where $x$ is an unconstrained rational number). For these models, the $Z-Z^{\prime}$ mixing vanishes at tree-level, which is desirable because it avoids the constraints from electroweak precision data.

For another one-parameter set of solutions, $\mathrm{U}(1)_{10+x \overline{5}}$, the fermion charges commute with the representations of the $\mathrm{SU}(5)$ group; it can arise from the breaking pattern $\mathrm{E}_{6} \rightarrow \mathrm{SU}(5) \times \mathrm{U}(1)_{\psi} \times \mathrm{U}(1)_{\chi}$ mentioned above. The specific cases $\mathrm{U}(1)_{\chi}, \mathrm{U}(1)_{\psi}$ and $\mathrm{U}(1)_{\eta}$ correspond to $x=-3,1,-1 / 2$ respectively.

Another set of solutions is $\mathrm{U}(1)_{d+x u}$, where the left-handed weak quark doublets are neutral under the new group, but the right-handed quark singlets carry a charge, with the ratio of the $u_{R}$ charge to the $d_{R}$ charge given by $-x$.

Finally, the most general generation-independent solution with no extra fermions that are charged under the SM group is $\mathrm{U}(1)_{q+x u}$, where the charges are a linear combination of the hypercharge $Y$ and $B-L$.

A model that is often used as a 'benchmark' in searches is the so-called Standard Model-like $Z^{\prime}$ model or sequential Standard Model (SSM) $Z^{\prime}$, for which the couplings of the $Z^{\prime}$ to the Standard Model fermions are identical to those of the $Z$ boson. This is an ad-hoc model; the extension is not, by it self, gauge invariant ${ }^{8}$, and is used mainly for reasons of convenience. However, it can be used as a starting point for model-independent limit setting, which is what is done here.

\footnotetext{
${ }^{8}$ There are models with extra dimensions in which a sequential $Z^{\prime}$ arises as a resonance of the Standard Model $Z$ [29].
} 


\begin{tabular}{|c|c|c|c|}
\hline limit on $M_{Z_{S S M}^{\prime}}\left(\mathrm{GeV} / c^{2}\right)$ & limit on $M_{Z_{x}^{\prime}}\left(\mathrm{GeV} / c^{2}\right)$ & Experiment/author & Type \\
\hline$>850$ & $>740$ & $\mathrm{CDF}[32]$ & $p \bar{p}$ with $Z^{\prime} \rightarrow e^{+} e^{-}$ \\
\hline$>780$ & $>640$ & $\mathrm{D} \varnothing[33]$ & $p \bar{p}$ with $Z^{\prime} \rightarrow e^{+} e^{-}$ \\
\hline$>680$ & (not given) & $\mathrm{D} \varnothing[34]$ & $p \bar{p}$ with $Z^{\prime} \rightarrow \mu^{+} \mu^{-}$ \\
\hline$>1305$ & $>545$ & DELPHI [30] & $e^{+} e^{-}$with $Z^{\prime} \rightarrow f \bar{f}$ \\
\hline$>1018$ & $>680$ & OPAL [31] & $e^{+} e^{- \text {with } Z^{\prime}} \rightarrow f \bar{f}$ \\
\hline$>1500$ & $>781$ & Cheung [35] & Combined electroweak precision \\
\hline
\end{tabular}

Table 2.3: Present $95 \%$ confidence limits on the mass of a hypothetical heavy neutral vector boson [16].

\section{Existing experimental constraints}

In general, any measurement of neutral currents is sensitive to the existence of a $Z^{\prime}$ through mixing and direct production. Generally speaking there are two ways in which the $Z^{\prime}$ can be constrained: indirectly, by fits to electroweak precision data, and directly, by a search for production and decay. The limits are summarized in table 2.3 .

Above the $Z$ resonance, but with $\sqrt{s}<M_{Z^{\prime}}$, strong indirect constraints on the existence of a $Z^{\prime}$ arise through measurements of asymmetries and leptonic and hadronic cross sections. The strongest of such constraints comes from the Large ElectronPositron (LEP) Collider, with the best limit on the mass of a hypothetical Standard Model-like $Z^{\prime}\left(M_{Z_{S S M}^{\prime}}\right)$ coming from a Delphi analysis at $\sqrt{s}=130-207 \mathrm{GeV}$ [30]. The most stringent constraint on $Z-Z^{\prime}$ mixing comes from electroweak precision fits at OPAL [31] at

$$
-0.00422<\theta_{Z-Z_{S S M}^{\prime}}<0.00091
$$

for a Standard Model-like $Z^{\prime}$.

The best limits on the direct production of a $Z^{\prime}$ with a large mass compared to $M_{Z}$ come from searches for dilepton resonances at the Tevatron proton-anti-proton collider, of which the present analysis is an example. While the limits on $M_{Z_{S S M}^{\prime}}$ from electroweak precision fits exclude a much larger range, this does not hold for general $Z^{\prime}$ models. As a comparison the limits on the mass of the aforementioned $Z_{\chi}^{\prime}$ are given as well.

\subsubsection{Little Higgs}

A special class of theories with larger symmetry groups are the little Higgs models [36]. Here, the symmetry groups and breaking pattern are chosen in a special way to protect the gauge hierarchy. In these models the Higgs particle is a pseudo-NambuGoldstone boson associated with a continuous global symmetry that is broken at a scale $\Lambda_{S} \sim 10-30 \mathrm{TeV}$. Its mass is protected because it is proportional to the breaking scale of this global symmetry. The radiative corrections to $m_{H}$ by Standard Model 
particles are cancelled by new particles with masses $\sim 1-3 \mathrm{TeV}$ that contribute with an opposite sign at the 1-loop level.

The "littlest" Higgs model [37] is the simplest example of such a theory. It has a continuous global symmetry group $\mathrm{SU}(5)$, and a $[\mathrm{SU}(2) \times \mathrm{U}(1)]_{1} \times[\mathrm{SU}(2) \times \mathrm{U}(1)]_{2}$ gauge symmetry group which is a subgroup of the global symmetry group $\mathrm{SU}(5)$.

At the scale $\Lambda_{S}$, the SU(5) multiplet $\Sigma$ acquires a non-zero vacuum expectation value

$$
\Sigma_{0} \propto f \cdot\left(\begin{array}{lll} 
& & \mathbb{I} \\
& 1 & \\
\mathbb{I} & &
\end{array}\right)
$$

where $\mathbb{I}$ is the $2 \times 2$ identity matrix. The vacuum expectation value $f$ is related to the breaking scale as $\Lambda_{S} \sim 4 \pi f$. This breaks the global symmetry group SU(5) down to $\mathrm{SO}(5)$, which results in 14 new particle degrees of freedom. Under the SM electroweak group these transform as a real singlet, a real triplet, a complex doublet and a complex triplet.

The 4 degrees of freedom in the real singlet and the real triplet become the longitudinal components of the gauge bosons associated with the broken part of the full gauge group, which thereby acquire a mass $M \sim g f \sim 1-3 \mathrm{TeV}$ (where $g$ is the gauge coupling strength). This breaks the gauge group $[\mathrm{SU}(2) \times \mathrm{U}(1)]_{1} \times[\mathrm{SU}(2) \times \mathrm{U}(1)]_{2}$ to the diagonal subgroup $\mathrm{SU}(2)_{L} \times \mathrm{U}(1)_{Y}$, which is the Standard Model electroweak gauge group.

The gauge and Yukawa couplings that break the $\mathrm{SO}(5)$ symmetry induce a potential of the form of equation (2.4) for the remaining pseudo-Nambu-Goldstone bosons. The neutral part of the complex doublet gets a non-zero vacuum expectation value $v$ which results in the electroweak symmetry breaking.

In addition, a new set of heavy fermions is introduced which have their couplings chosen such that their quadratic corrections to the Higgs mass cancel those of the top/anti-top quarks.

One (or in some little Higgs models, two) of the new heavy gauge bosons is a new neutral massive gauge boson, i.e., a $Z^{\prime}$, associated with the extra $\mathrm{SU}(2)$ or $\mathrm{U}(1)$ gauge groups; there is also a $W^{\prime}$, associated with the broken part of the subgroup. The $Z^{\prime}$ couples left-handedly and universally to all Standard Model fermions with a coupling strength $g_{V}=-g_{A} \sim \frac{g}{2} \cot \theta T_{3}$, where $\theta$ is a mixing angle and $T_{3}$ is the charge under the new group. The production cross-section through a Drell-Yan process is then proportional to the partial width

$$
\Gamma\left(q q^{\prime} \rightarrow Z^{\prime}\right)=\frac{C}{12 \pi}\left(g_{V}^{2}+g_{A}^{2}\right) M_{Z^{\prime}} \propto \cot ^{2} \theta
$$

Figure 2.5 shows the dependence of the branching ratios on the mixing angle [38]. For $\cot \theta \gtrsim 0.5$ the branching ratio to leptons is nearly independent of $\cot \theta$. For $\cot \theta \lesssim$ 0.5 the BR to fermion-anti-fermion pairs drops sharply and other decay channels dominate. 


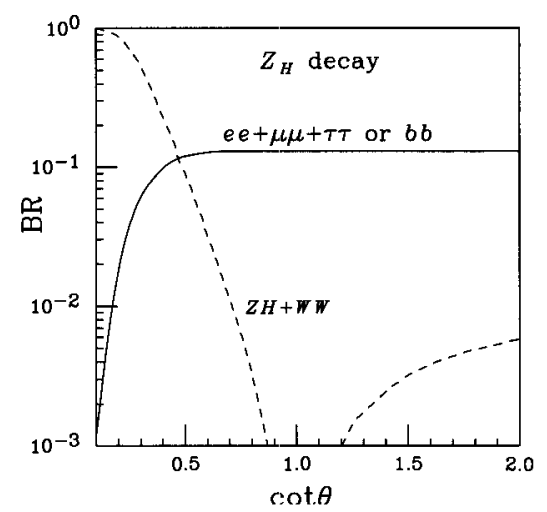

Figure 2.5: Branching ratios of the $Z^{\prime}$ into SM particles in the littlest Higgs scenario, as a function of $\cot \theta$, neglecting final-state mass effects [38].

\subsubsection{Extra dimensions}

A different approach is taken by a number of models with extra (space) dimensions. The primary motivation for this approach is again to resolve or hide the gauge hierarchy.

The central idea is that gravity appears to be weak (i.e., the Planck mass appears to be large) because there exist one or more extra space dimensions in which only gravity propagates, i.e. gravity waves propagate in a $(3+n)$ dimensional space but the Standard Model fields are localized on a 3 dimensional surface in the full $3+n$ dimensional space ${ }^{9}$.

In the models discussed here, the extra space dimensions are very small compared to the 4 normal dimensions, so that the effective strength of gravity at macroscopic scales can remain unmodified.

\section{Large extra dimensions}

The first proposal $[39,40]$ that exploits this effect, was the theory of large extra dimensions or the $\mathrm{ADD}$-mode ${ }^{10}$. Here, the $n \geq 1$ extra dimensions are chosen to be compactified to a finite volume, and the 4 dimensional metric does not depend on the extra coordinates. This means the Einstein-Hilbert action in $4+n$ dimensions,

$$
S\left(g_{4+n}\right)=\frac{1}{2} \bar{M}_{4+n}^{2+n} \int d^{4} x \int d^{n} y \sqrt{-g_{4+n}} \mathcal{R}\left(g_{4+n}\right)
$$

(where $\bar{M}_{4+n}$ is the (reduced) $4+n$-dimensional Planck mass $\left(\bar{M}_{4+n}=M_{4+n} /(8 \pi)^{\frac{1}{2+n}}\right)$, $g_{4+n}$ is the determinant of the $4+n$-dimensional Lorentz metric and $\mathcal{R}$ is the scalar

\footnotetext{
${ }^{9}$ In the minimal models discussed here; more generally, they may propagate in all dimensions but be suppressed outside the 3 dimensional surface to satisfy experimental contraints.

${ }^{10}$ No limits are derived for this model in this thesis, but it is summarized here by way of introduction.
} 
curvature) gives rise to an effective action for the 4-dimensional gravity field $g$ of the form

$$
S(g)=\frac{1}{2} V_{n} \bar{M}_{4+n}^{2+n} \int d^{4} x \sqrt{-g} \mathcal{R}(g)
$$

where the integral over the extra dimensional coordinates $y^{1 \ldots n}$ has simply become the volume of the extra dimensions, $V_{n}$. From this, one can conclude ${ }^{11}$ that the Planck mass in 4 dimensions, $\bar{M}_{\mathrm{Pl}}$ is related to the one in $4+n$ as

$$
\bar{M}_{\mathrm{Pl}}^{2}=V_{n} \bar{M}_{4+n}^{2+n}
$$

Therefore, the true Planck scale $\bar{M}_{4+n}$ can be of the order of the electroweak scale $m_{H} \sim 1 \mathrm{TeV}$ if the volume of the extra space is large enough. For compactification on a torus so that $V_{n}=(2 \pi R)^{n}$ where $R$ is the radius of the extra dimension, one gets

$$
R^{-1} \sim 2 \pi \bar{M}_{4+n}\left(\bar{M}_{4+n} / \bar{M}_{P l}\right)^{2 / n}
$$

For $n=2$ and $\bar{M}_{4+n} \sim 1 \mathrm{TeV}$ this works out to $R \sim 1 \mathrm{~mm}$ which is just excluded by (among others) direct measurements of gravity; for $n>2$ the size of the extra dimensions is too small to be detected by precision gravity experiments. For a review of direct measurements, see [42].

Although the gauge hierarchy has apparently disappeared from the theory, requiring that $\bar{M}_{4+n} \sim 1 \mathrm{TeV}$ does give rise to a new hierarchy, namely that $V_{n}^{-1} \ll M_{4+n}^{n}$. This hierarchy has to be stabilized in some way, but this would supposedly rely on some dynamical theory of quantum gravity.

\section{Warped extra dimensions}

Another approach is taken by the Randall-Sundrum (RS) model [43], where the geometry of the extra dimensions is chosen such that no new hierarchy arises. The extra dimensions are compactified to an $S_{1} / \mathbb{Z}_{2}$ orbifold, i.e., a circle with opposite points identified. The fixed points of $\mathbb{Z}_{2}$ on $S_{1}$ are the location of branes with equal but opposite tension; the Standard Model fields are localized on the negative tension brane, whereas gravity is localized on the 'hidden' positive tension brane. The solution of the Einstein equations in vacuum gives the following solution for the metric describing the space,

$$
d s^{2}=e^{-2 k|y|} d x_{\mu} d x_{\nu} \eta^{\mu \nu}-d y^{2}
$$

with $y \in[0, \pi R]$, the Standard Model brane located at the $y=\pi R$ and the other at $y=0$. The curvature of this 5-dimensional space is $R_{5}=-20 k^{2}$, i.e., it has constant negative curvature; such spaces are called "Anti-deSitter" spaces. Unlike in the ADD model, the 4-dimensional metric depends on the extra coordinate $y$.

\footnotetext{
${ }^{11}[41]$ presents a physical and more intuitive derivation using Gauss' law for gravitation.
} 
The action for the Higgs potential (see (2.4)) in 5 dimensions, after integrating over the $y$-coordinate, looks like

$$
S=\int d x^{4}\left(e^{-2 \pi k R} \eta^{\mu \nu} \partial_{\mu} H^{\dagger} \partial_{\nu} H-\lambda e^{-4 \pi k R}\left(H^{2}-v^{2}\right)^{2}\right)
$$

where $v$ is the vacuum expectation value of the Higgs field. Rewriting this in the canonical form by substituting $H \rightarrow e^{\pi k R} h$ we get

$$
S=\int d x^{4}\left(\eta^{\mu \nu} \partial_{\mu} h^{\dagger} \partial_{\nu} h-\lambda\left(h^{2}-e^{-2 \pi k R} v^{2}\right)^{2}\right)
$$

The effective value of the Higgs v.e.v. for the 4-dimensional theory is then $v^{\prime}=$ $e^{-\pi k R} v$. The effective 4-dimensional Planck scale $\bar{M}_{\mathrm{Pl}}$ is related to the true 5dimensional Planck scale $\bar{M}_{5}$ as

$$
\bar{M}_{\mathrm{Pl}}^{2}=\left(1-e^{-2 \pi k R}\right) \bar{M}_{5}^{3} / k
$$

This means that large hierarchies can be avoided, i.e., $1 \mathrm{TeV} \sim \bar{M}_{\mathrm{Pl}} \sim \bar{M}_{5} \sim v \sim k$ if

$$
k R \sim 11
$$

This has been shown to be a 'natural' hierarchy, which can be realized without finetuning. Therefore, in the RS model the hierarchy problem truly disappears; all masses are of the order $\bar{M}_{\mathrm{Pl}}$, but on the Standard Model brane they appear to be $\sim 1 \mathrm{TeV}$ because they are scaled by a "warp" factor $e^{-\pi k R} \sim \frac{1 \mathrm{TeV}}{\bar{M}_{\mathrm{P} 1}}$.

The next question is what the effective $4 \mathrm{D}$ action for the 5-dimensional graviton looks like. Although the graviton is described by a 5D massless spin-2 field, qualitatively the effective $4 \mathrm{D}$ action is similar to that of a $5 \mathrm{D}$ massless scalar [44]. The action for such a field is

$$
S[\Phi]=\int d x^{4} \int_{0}^{\pi R} d y\left(e^{-2 k|y|} g^{\mu \nu} \partial_{\mu} \Phi \partial_{\nu} \Phi-\partial_{y} \Phi \partial_{y} \Phi\right)
$$

Because in the 5th coordinate $y$ the field is constrained by the geometry of the extra dimension to be periodic, using Fourier decomposition the $y$-dependence of $\Phi$ can be written in terms of an orthonormal basis of periodic functions $\chi_{n}(y)$ as $\Phi=$ $\sum_{n} \chi_{n}(y) \phi_{n}(\mathrm{x})$ with $\int_{0}^{\pi R} d y \chi_{n} \chi_{m}=\delta_{n m}$.

The equations of motion for the fields $\chi_{n}(y)$ derived from this action can then be written as

$$
-e^{k \pi y} \partial_{y}\left(e^{-2 k \pi y} \partial_{y} \chi_{n}\right)=m_{n}^{2} \chi_{n}
$$

which have a solution in terms of the Bessel functions $J_{2}, Y_{2}$, with

$$
m_{n}=x_{n} k e^{-k \pi R}
$$


where $x_{n}$ is the $n$-th zero of the Bessel function $J_{1}, J_{1}\left(x_{n}\right)=0, x_{n}=0,3.38,7.02 \ldots$ The effective $4 \mathrm{D}$ action for the fields $\phi_{n}$ is then

$$
\int d x^{4} \frac{1}{2} \sum_{n}\left(\partial^{\mu} \phi \partial_{\mu} \phi-m_{n}^{2} \phi^{2}\right)
$$

Thus, this theory describes one massless scalar field $\phi_{0}$ and an infinite number of massive scalar fields $\phi_{n}$. From equation (2.9) we see that the masses $m_{n}$ are also of the order of $\sim 1 \mathrm{TeV}$.

These scalar fields are the analogues of the RS gravitons $G^{[n]}$; the massless graviton $G^{[0]}$ is the normal graviton that mediates gravity and the rest are massive spin-2 resonances. The theory has 2 free parameters; these are usually taken to be the mass of the lightest massive graviton, $M_{G^{[1]}}$ and $\frac{k}{\bar{M}_{\mathrm{Pl}}}$.

A realization of the RS model in string theory restricts the value of $\frac{k}{\overline{M_{\mathrm{Pl}}}}$ to be greater than 0.01 (see [45]). Requiring the curvature of the extra dimension to be less than the 5-dimensional Planck scale restricts $\frac{k}{M_{\mathrm{P} 1}}$ to be less than $\approx 0.1$ (see $[46,47]$ ).

The width of the $n$-th resonance is given by

$$
\Gamma_{n}=\rho m_{n} x_{n}\left(\frac{k}{\bar{M}_{\mathrm{Pl}}}\right)^{2}
$$

where $\rho$ is a constant that only depends on the number of decay channels that are open.

For $\frac{k}{\bar{M}_{\mathrm{Pl}}}$ less than 0.1 the lightest massive resonance has a width of not more than a couple of $\mathrm{GeV}$.

The RS graviton fields $h_{\alpha \beta}^{(n)}$ interact with the Standard Model fields by coupling to the energy-momentum tensor,

$$
\mathcal{L}=-\frac{1}{e^{-k \pi R} \bar{M}_{\mathrm{Pl}}} T^{\alpha \beta} \sum_{n=1}^{\infty} h_{\alpha \beta}^{(n)}
$$

At the Tevatron, the resonances are produced through $q \bar{q} \rightarrow G^{[n]}$ and $g g \rightarrow G^{[n]}$ and can decay to $G^{[n]} \rightarrow \ell^{+} \ell^{-}, g g, \gamma \gamma, q \bar{q}$. Discovering and measuring the properties of the first resonance $G^{[1]}$ would allow the determination of all parameters in the model [45].

\section{Existing experimental constraints}

The most stringent constraint on the presence of a RS graviton comes from a direct search at $\mathrm{D} \varnothing$ with $1 \mathrm{fb}^{-1}$ of data in the combined $e^{+} e^{-}$and $\gamma \gamma$ channels [48], which excludes a massive graviton with $M_{G^{[1]}}<300 \mathrm{GeV} / c^{2}$ for $\frac{k}{M_{\mathrm{Pl}}}=0.01$ and $M_{G^{[1]}}<$ $900 \mathrm{GeV} / c^{2}$ for $\frac{k}{M_{\mathrm{Pl}}}=0.1$. 


\section{Chapter 3}

\section{Experimental Setup}

\subsection{Tevatron}

The Fermilab Tevatron Collider is the highest energy collider currently in operation. The first large scale super-conducting synchrotron, it was commissioned in 1983 in proton-only fixed target mode, but since 1985 it operates as a proton-anti-proton $(p \bar{p})$ collider. The first physics run ("Run 0") took place between 1988 and 1989 with only one of the currently two detectors in operation, namely CDF ("Collider Detector at Fermilab"). The D $\varnothing$ detector started taking data in Run I of the Tevatron, which took place between 1992-1996. During this period of data-taking the center-of-mass energy was $1.8 \mathrm{TeV}$ and the total integrated luminosity recorded by each experiment was $120 \mathrm{pb}^{-1}$. After a five year shutdown, during which the accelerator chain and both detectors were upgraded, the Tevatron resumed operation and data-taking in early 2001 at a center-of-mass energy which was approximately $9 \%$ higher compared to that of Run I. Both beams now have an energy of $980 \mathrm{GeV}$, resulting in a center-of-mass energy of $\sqrt{s}=1.96 \mathrm{TeV}$ for Run II. The numbers of bunches of protons and antiprotons used in the Tevatron were increased from 6 to 36 and the beam intensities were much higher than in Run I. This led to considerably higher instantaneous luminosities. Run II began in early 2001 and is split into two parts, Run IIa and Run IIb. Run IIa finished in April 2006, and all data used in this thesis were collected during this run. Both detectors were upgraded to operate at the higher instantaneous luminosities expected in Run IIb, which began in June 2006.

Figure 3.1 shows the peak instantaneous luminosity achieved during Run IIa and the beginning of Run IIb up till autumn 2007; figure 3.2 shows the weekly and run integrated luminosity.

A chain of accelerators perform proton and anti-proton production, pre-acceleration, accumulation and storage. Figure 3.3 shows a schematic overview of the accelerator chain. An overview of the (anti-)proton production and acceleration processes will be given in the following sections. A more detailed description can be found in [50].

The final acceleration takes place in the Tevatron, which is a circular super-conducting magnet synchrotron with a radius of about $1 \mathrm{~km}$ that accelerates protons and anti- 


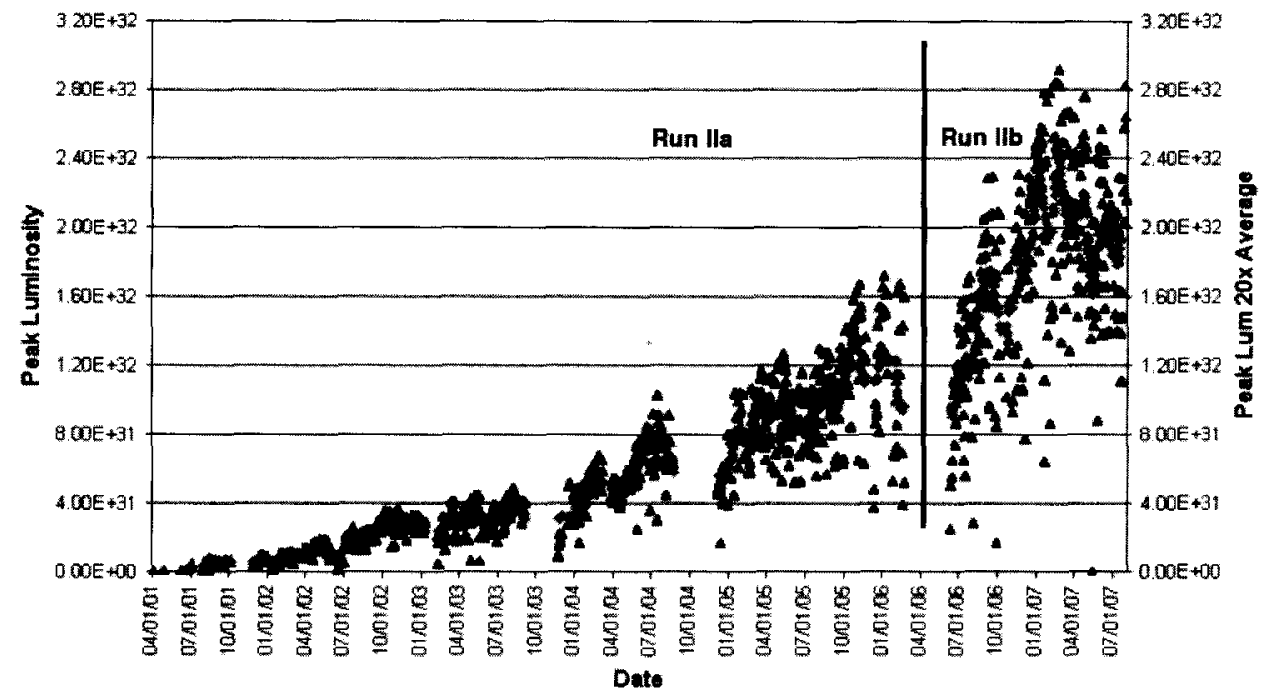

- Peak Luminosty + Peak Lum $20 x$ Average

Figure 3.1: Peak instantaneous luminosity (i.e. the luminosity at the beginning of a store) delivered by the Tevatron during Run IIa and the beginning of Run IIb [49] and the same averaged over 20 stores.

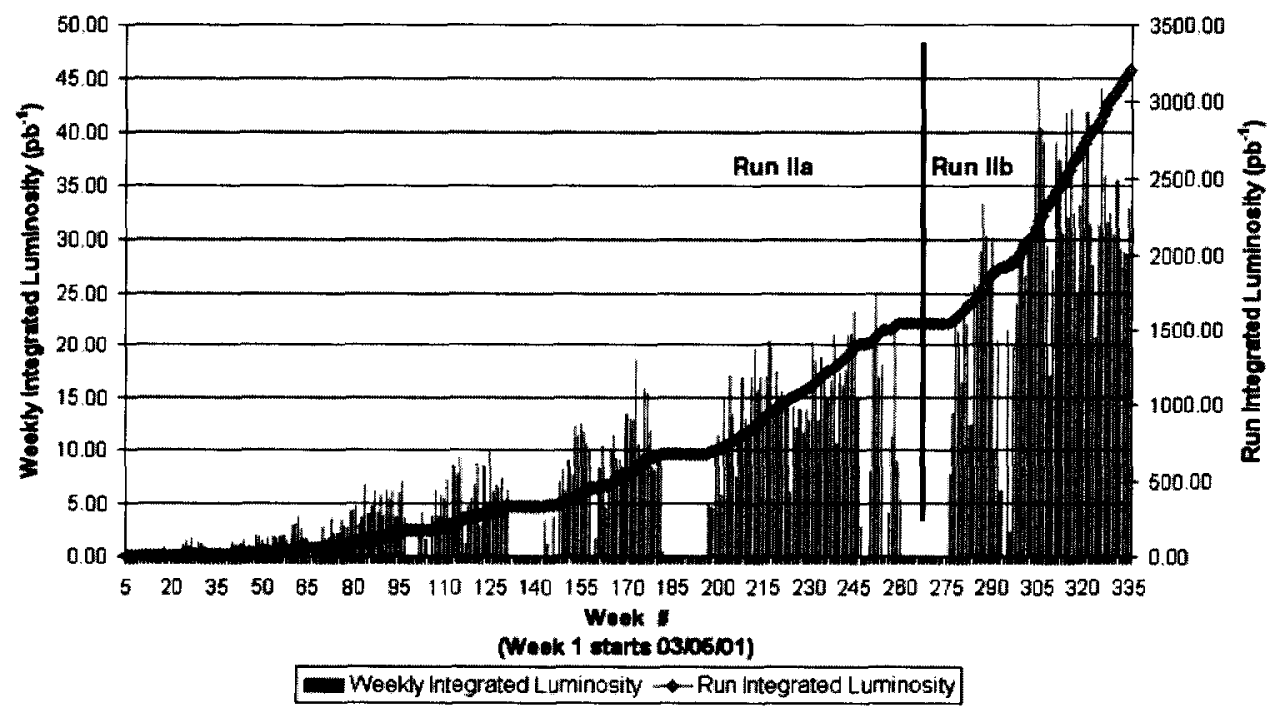

Figure 3.2: Tevatron weekly and Run II Integrated Luminosity [49]. 


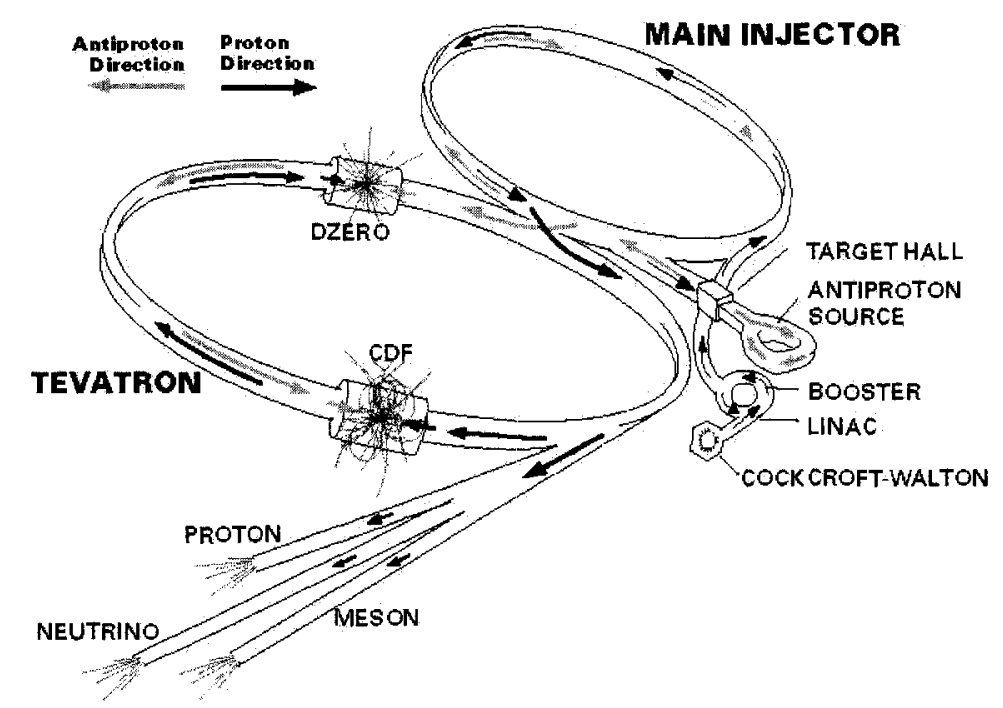

Figure 3.3: The Fermilab accelerator chain.

protons from 150 to $980 \mathrm{GeV}$. The 4.2 Tesla dipole and quadrupole magnets are cooled to 4.6 Kelvin with liquid helium. During physics data taking there are 36 bunches of protons and 36 bunches of anti-protons going around the ring, arranged into three 'super-bunches' respectively, consisting of 12 bunches each. Within a super-bunch, the bunches are spaced $396 \mathrm{~ns}$ apart. The beams are accelerated to $980 \mathrm{GeV}$ each in the same beam pipe, but in opposite directions, and are brought to collision at two designated points in the accelerator, called $\mathrm{B} 0$ and $\mathrm{D} \varnothing$, which are the locations of the $\mathrm{CDF}$ and $\mathrm{D} \varnothing$ detectors respectively

The Tevatron Collider is located on the Fermilab site in the west suburbs of Chicago, Illinois.

\subsubsection{Proton Source}

The pre-accelerator produces negatively-charged hydrogen ions $\left(\mathrm{H}^{-}\right)$and boosts them to an energy of $750 \mathrm{keV}$ for insertion into the linac. $\mathrm{H}^{-}$ions are easily produced with a surface plasma source (SPS). SPS devices [51] produce $\mathrm{H}^{-}$ions through the interaction of plasmas, consisting of protons, ionized hydrogen molecules, or heavier positive ions, with cathode surfaces containing absorbed hydrogen atoms. $\mathrm{H}^{-}$ions are pulled through the plasma from the cathode to the anode for collection. The magnetron source used at the Tevatron is a particular type of SPS .

Before insertion into the linac, the $\mathrm{H}^{-}$ions are accelerated to $750 \mathrm{keV}$ with a CockcroftWalton type generator [52]. This is a device which charges capacitors in parallel using an $\mathrm{AC}$ voltage source, and then discharges them in series through the use of diodes.

The Linac [53] is a two-stage linear accelerator, which accelerates the $750 \mathrm{keV} \mathrm{H}^{-}$ions up to $400 \mathrm{MeV}$. The first stage, which was part of the original $200 \mathrm{MeV}$ Linac (built 
in 1971), accelerates the ions to $116 \mathrm{MeV}$. The second stage, a more modern, sidecoupled accelerator that replaced the high energy portion of the original accelerator in 1993, accelerates the ions up to $400 \mathrm{MeV}$.

From the Linac, the ions can go to one of three destinations: two are dump lines that allow for measurements of either the momentum spread or the transverse emittance, and the third line goes to the Booster [54].

The Booster is the first synchrotron that the particles encounter, consisting of a sequence of dipole and quadrupole magnets and $17 \mathrm{RF}$ cavities in a 151 meter circle that accelerate particles from $400 \mathrm{MeV}$ to $8 \mathrm{GeV}$. Injection is done via a multiturn charge-exchange injection [55], which is the reason that $\mathrm{H}^{-}$ions are used in the beginning of the accelerator chain. After debunching the $\mathrm{H}^{-}$ions from the Linac to minimize their momentum spread, the $\mathrm{H}^{-}$beam is merged over multiple turns with the proton beam that is already in the booster, and the electrons are stripped by a carbon foil.

The second synchrotron is the Main Injector [56], a ring of $3.3 \mathrm{~km}$ circumference with $20 \mathrm{RF}$ cavities for particle acceleration and a series of dipole and quadrupole magnets for beam focusing and steering. The Main Injector not only accelerates protons from the booster (or anti-protons from the anti-proton source) from $8 \mathrm{GeV}$ to $150 \mathrm{GeV}$ for injection into the Tevatron, but it also accelerates protons to $120 \mathrm{GeV}$ for use by the anti-proton source in creating $8 \mathrm{GeV}$ anti-protons. The Main Injector also has uses for neutrino experiments, but these will not be discussed here.

During normal collider operations, at first the protons from the Booster are transferred to the Main Injector. Seven of the $848 \mathrm{GeV}$ bunches of the Booster are transferred in a single batch to the Main Injector, accelerated to $150 \mathrm{GeV}$ and then coalesced into a single super-bunch. This super-bunch is transferred to the Tevatron and the process is repeated until 36 proton bunches have been transferred to the Tevatron. Then $8 \mathrm{GeV}$ anti-protons are transferred from the anti-proton source to the Main Injector. Four groups of anti-protons are transferred at a time, each group containing several bunches. These groups are coalesced into 4 bunches, accelerated to $150 \mathrm{GeV}$, and transferred to the Tevatron. This is done 9 times for a total of 36 anti-proton bunches.

\subsubsection{Anti-proton source}

For anti-proton production [57], a full batch of 84 proton bunches is accelerated to $120 \mathrm{GeV}$ in the Main Injector. This beam is diverted to a nickel target, producing a shower of secondary particles. A dipole magnet acts on the resulting particles, bending the negative particles which have energies of $8 \mathrm{GeV}$ into another line. At this point, the momentum spread is large. It is necessary to reduce this momentum spread prior to injection into the Tevatron. This is done through bunch rotation and adiabatic debunching in the Debuncher, a triangular $8 \mathrm{GeV}$ synchrotron. From there, the anti-protons are 'stacked' in the Accumulator, another $8 \mathrm{GeV}$ synchrotron.

They are placed in an orbit which is $\sim 80 \mathrm{~mm}$ outside the orbit of anti-protons that may already exist in the Accumulator, captured in 84 bunches, moved to the end of an existing stack and slowed down through stochastic cooling (where the displacement or 
energy difference of an ensemble of particles relative to the ideal orbit is "picked up" at one point in the ring, amplified and corrected by fast electric pulses in a "kicker" at another point in a negative feedback loop) until all bunches are in the same orbit as the original stack. This process takes about 30 minutes and is repeated until the desired anti-proton stack in the Accumulator is achieved.

The stacking efficiency of the Accuinulator depends on the amount of already stacked anti-protons. Therefore some fraction of the particles are transferred to the Recycler [58], which was designed to store anti-protons. The Recycler has no special features except that it uses mostly permanent magnets. It stores anti-protons from the Accumulator to allow a higher production and stack rate in the latter, and is located along the ceiling of the tunnel of the Main Injector. The Recycler cools the anti-protons stochastically, similarly to the Accumulator, until the intensity reaches $200 \times 10^{10}$ antiprotons in the Recycler. At this point, stochastic cooling is inefficient and electron cooling is used to further cool the anti-protons. This works by transferring momentum from the anti-protons to relatively "cooler" electrons, which are driven at the same energy as the anti-protons and injected in a very concentrated beam on top of the anti-protons. The anti-protons transfer momentum to the electrons, giving cooled anti-proton bunches. The electron beam is removed when cooling is complete. The Recycler stores anti-protons until needed for injection into the Tevatron.

Only after the transfer of the 36 proton bunches from the Main Injector to the Tevatron main ring are the anti-protons transferred; first from the Accumulator and the Recycler to the Main Injector and then after acceleration to $150 \mathrm{GeV}$, to the Tevatron. The reason for transferring the anti-protons after the protons is to keep the anti-protons safe as long as possible, because of the significantly larger operating expense to produce anti-protons compared to the more simple proton acceleration. As soon as all 36 anti-proton bunches are in the Tevatron beam pipe, the energy is increased to $980 \mathrm{GeV}$ per particle during normal operations. The 36 proton and 36 anti-proton bunches are grouped in three superbunches with a $7 \mu$ s separation. The bunch to bunch separation time is $396 \mathrm{~ns}$. A proton bunch contains about $2.4 \times 10^{11}$ protons, the anti-proton bunch about 2 to 5 times less, depending on the number of available anti-protons - the number of particles per bunch is limited because of the repelling electric forces between them. The rms bunch length is $\sigma=37 \mathrm{~cm}$. The beams are normally stored for up to 36 hours.

After acceleration, the low-beta magnets (see figure 3.4 for their position relative to the $\mathrm{D} \varnothing$ detector) are ramped up, focusing the proton and anti-proton beams into the interaction zones in the $\mathrm{D} \varnothing$ and $\mathrm{CDF}$ detectors. The electric fields are then removed from the separators near the collision halls, allowing collisions to occur.

As the beams are brought into collision, the beam halo must be reduced to avoid damage to detectors near the beam and also to reduce background. This is done with scrapers, which are simply steel blocks placed very near the beam to remove halo particles through interaction. A second block is used, slightly farther away from the beam, to block particles that are created through interaction of beam halo with the first block. After scraping is complete, luminosity monitoring and physics data taking begins.

During a store, the beam intensity and therefore the luminosity drops exponentially, 
since the particle loss rate is approximately proportional to the particle flux. The old beams are dumped if the Tevatron luminosity drops below $10^{31} \mathrm{~cm}^{-2} \mathrm{~s}^{-1}$ or as soon as enough anti-protons are stacked in the Accumulator or Recycler.

Alternatively, the beam circulation can be stopped unintentionally if a magnet quenches. A quench is the local break-down of superconductivity in a magnet coil, triggered by, e.g., a temperature fluctuation of the liquid helium coolant. This can result in an accidental particle loss; the magnetic field in the quenched magnet will break down. Since this is in general slow compared to the circulation frequency, the beams can be dumped in time to avoid further damage. An unnoticed change of the beam orbit can result in the quenching of a large number of magnets. During ' $01-^{\prime} 03$ the average number of quenches per month was about 15 ; in '04-' 06 this number dropped to around 7 . The typical recovery time is a few hours.

\subsection{The $\mathrm{D} \varnothing$ Detector}

The $\mathrm{D} \varnothing$ detector [59] (figure 3.4) is a multipurpose detector designed to detect particles arising from proton-anti-proton collisions occurring at the $\mathrm{D} \varnothing$ intersection point of the Tevatron. It operates with an average data-taking efficiency of around $85 \%$ (see figure 3.5).

Its outer dimensions are $20 \times 12 \times 12$ meters and it weighs about 5500 tons. It has a symmetric design of concentric sub-detectors centered around the collision point. The innermost detector is the central tracking system which consists of a silicon microstrip tracker (SMT) and central fiber tracker (CFT). A 2 Tesla solenoid provides a field which allows determination of the momentum of charged particles through the bending radius of their reconstructed tracks. The central tracking system is enveloped by a sampling calorimeter. Outside the calorimeter are the muon spectrometers with a toroid magnet. The sub-detectors are described in more detail in the following sections.

\section{Coordinate System}

The three-momenta of particles or jets reconstructed in the detector are usually described in a Cartesian coordinate system oriented with the " $z$ "-axis pointing along the proton beam, the " $y$ "-axis pointing vertically upwards and the " $x$ "-axis pointing horizontally away from the center of the Tevatron ring. Alternatively, cylindrical coordinates are used with the symbols $\phi$ and $\theta$ denoting the azimuthal and polar angles respectively, with $\phi=0$ pointing along the $x$-axis and $\theta=0$ pointing along the beam pipe in the $+z$ direction. If the coordinate system is centered at the center of the detector one speaks of "detector" coordinates, while if they are centered at the hard interaction (primary) vertex one speaks of "physics" coordinates.

A useful parameter for physics analyses is the pseudo-rapidity, $\eta$, defined as

$$
\eta=-\log \left(\tan \left(\frac{\theta}{2}\right)\right)
$$




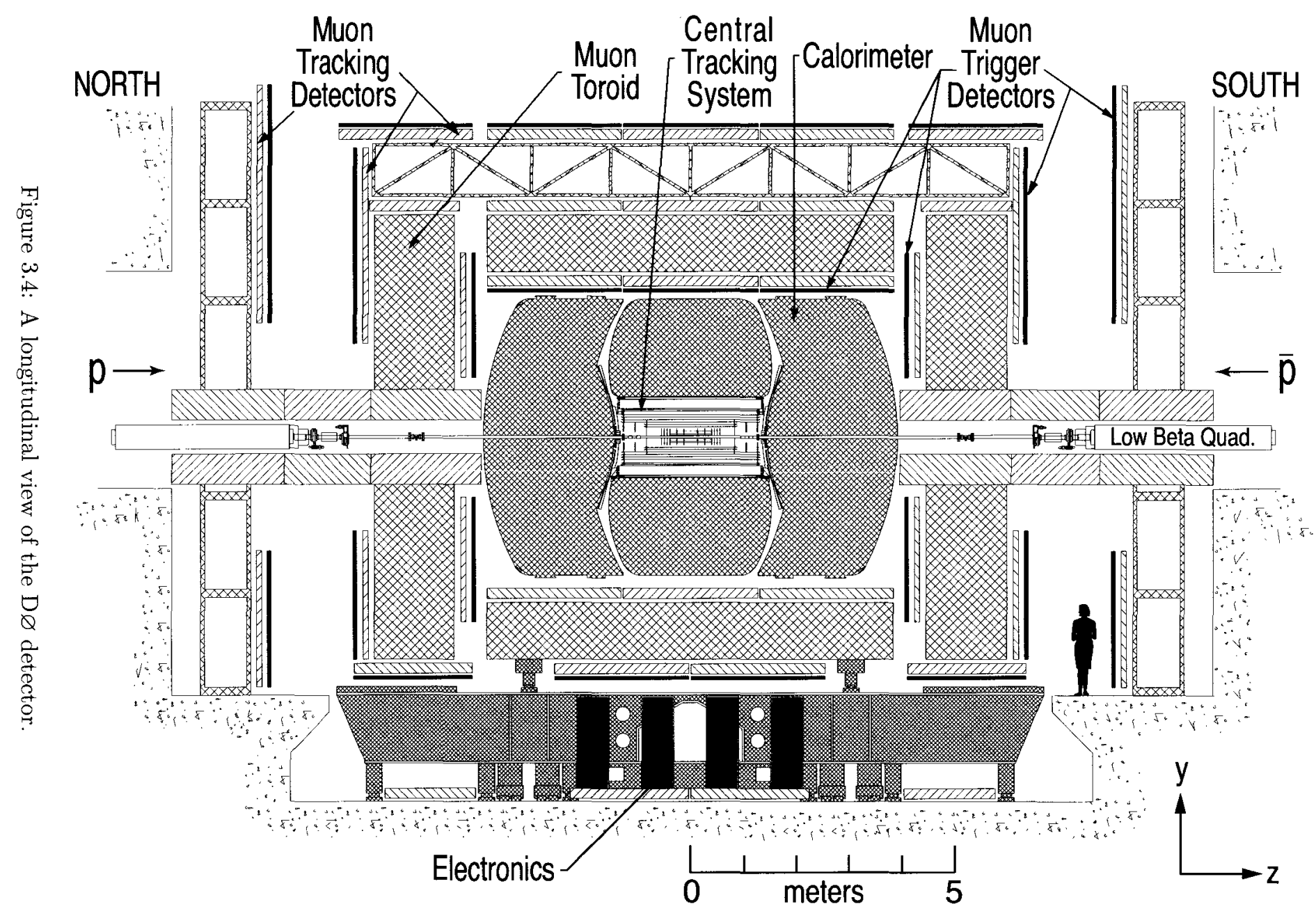

iv

.

검

$\square$

검

स्त

$\bar{p}$

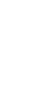




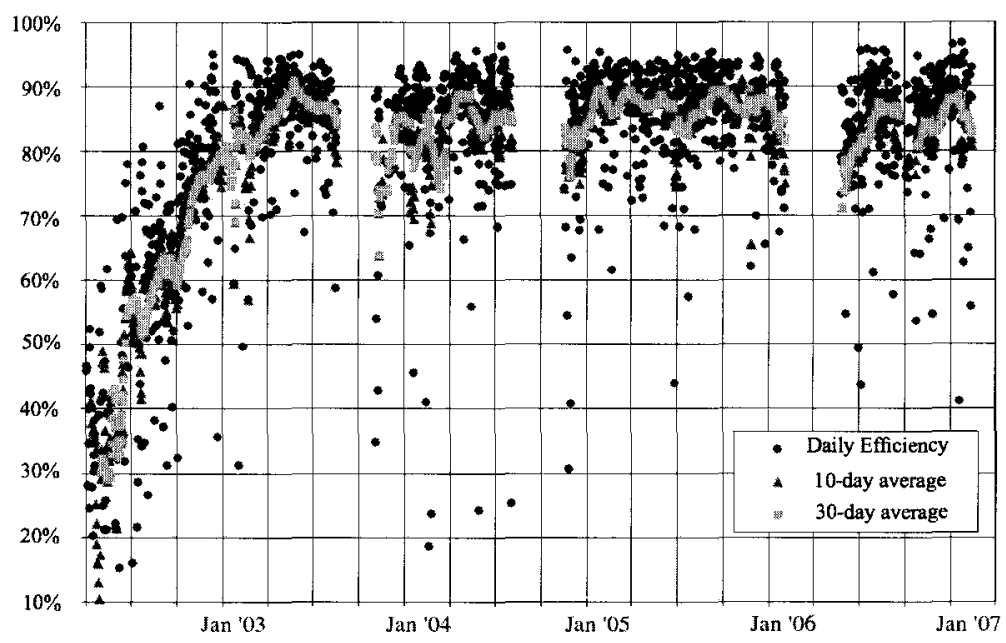

Figure 3.5: Daily, 10-day and monthly data-taking efficiency for Run IIa (until April 2006) and the beginning of Run IIb; the data used in this thesis were collected during Run IIa. The data-taking efficiency is defined as the ratio of the recorded luminosity to the delivered luminosity.

which approaches the true rapidity $y=\frac{1}{2} \ln \frac{E+p_{z}}{E-p_{z}}$ for high energies where the masses of particles become negligible and $E \approx|\vec{p}|$. This is a convenient parameter for hadron colliders because the shapes of rapidity distributions are invariant under Lorentz boosts in the $z$-direction, and the longitudinal momentum of the initial partons in a hard scattering is not known.

In addition, since the particle multiplicity is approximately constant as a function of the rapidity in the parton-parton c.o.m. frame, $y_{\mathrm{cm}}=\frac{1}{2} \ln \frac{x_{1}}{x_{2}}$ (i.e., in the approximation that on average the momentum fraction $x_{i}$ carried by a hard-interacting parton is equal for protons and anti-protons) the multiplicity of high energy particles is approximately constant in lab-frame rapidity, too.

\section{Interaction region}

The interaction volume of the proton and anti-proton beams has an (approximately) ellipsoidal shape with a typical width in the $x$ and $y$ directions of $\sigma_{x, y} \approx 30 \mu \mathrm{m}$ and a length in the $z$-direction of $\sigma_{z} \approx 25 \mathrm{~cm}$ (consistent with a bunch length of $\sim 37 \mathrm{~cm}$ ). The position of the interaction region ellipsoid is not completely stable, because of changes of the beam optics due to maintenance or upgrades, or during stores, which can cause the interaction region to shift by distances on the order of $100 \mu \mathrm{m}$. 


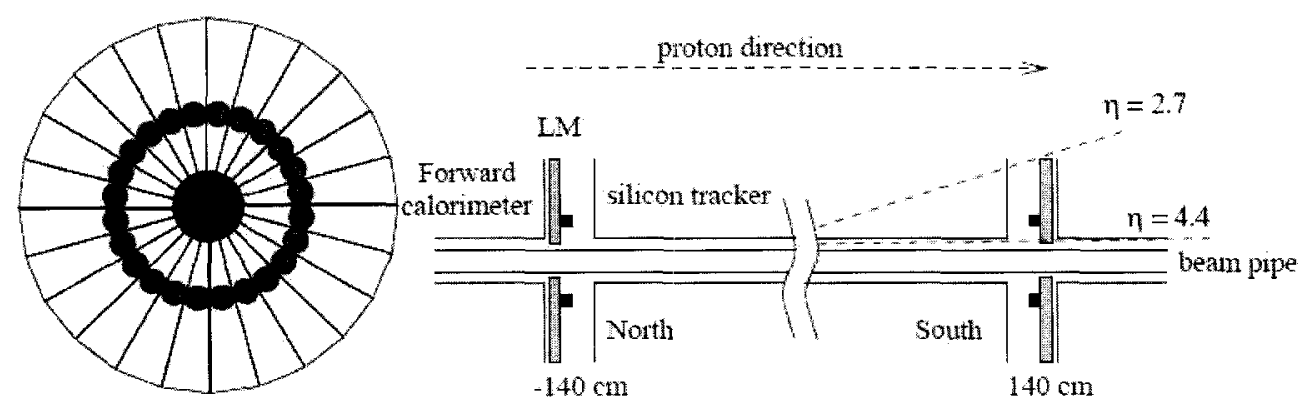

Figure 3.6: Luminosity detector with photo multiplier tubes.

\subsubsection{Trigger system}

Every second 1.7 million bunch crossings occur in the Dø detector, but the data of only around 50 of these can be written to tape. The data of one bunch crossing is called $^{1}$ an "event". Most collisions are elastic collisions or low-energy interactions (called minimum bias interactions), and the purpose of the trigger system is to filter these out and retain only data from hard interactions that are interesting in some way.

The $\mathrm{D} \varnothing$ detector uses three layers of triggering. The Level 1 trigger system must reduce the incoming rate of $1.7 \mathrm{MHz}$ down to a design output rate of $10 \mathrm{kHz}$, with $3.3 \mu \mathrm{s}$ of processing time per event. It is a hardware-based system because of the high input data rate it must handle and the tight timing required, and uses a reduced form of the detector readout, using only information from the luminosity system, the central fiber tracker, the calorimeter and the muon chambers.

The Level 2 trigger system reduces the rate by a factor of 10 to $1 \mathrm{kHz}$. It is based on both special hardware and embedded microcontrollers. It uses the Level 1 results with the addition of data from the silicon microstrip detector. Since the L2 trigger system has more time to spend on the events than the L1 trigger on the events, it can implement more sophisticated algorithms and make more correlations between the detectors.

The Level 3 trigger system generally reduces the accept rate to approximately 50 $\mathrm{Hz}$, which is written to tape, but the accept rate can increase to $100 \mathrm{~Hz}$ during high instantaneous luminosity times at the beginning of a store. The L3 trigger is softwarebased, running on a computer farm, with access to the full information of the events and does partial reconstruction of the events with algorithms similar to those used offline. 


\subsubsection{Luminosity monitors}

The Luminosity Monitor $(\mathrm{LM})[60]$ is used to determine the luminosity $(\mathcal{L})$ at the $\mathrm{D} \varnothing$ interaction point by detecting inelastic $p \bar{p}$ collisions ${ }^{2}$. The luminosity detector consists of two arrays of 24 plastic scintillation counters and photo multiplier tubes (PMT). A schematic drawing of an array is shown in figure 3.6 (left). The location of the PMT is marked by solid dots. The luminosity detectors are located at $z= \pm 140 \mathrm{~cm}$, figure 3.6 (right), between the silicon tracker and the forward calorimeter. The scintillation counters are $15 \mathrm{~cm}$ long and cover a pseudo-rapidity region of $2.7 \leq|\eta| \leq 4.4$. To detect an event of the elastic and inelastic scattering reference process, both luminosity detectors have to fire. Background from beam-halo is suppressed by requiring that the $z$-coordinate of the interaction vertex be within $100 \mathrm{~cm}$ of the detector center. The vertex $z$-coordinate $z_{\text {vertex }}$ is calculated as $z_{\text {vertex }}=c / 2\left(t^{-}-t^{+}\right)$, where $t^{ \pm}$is the time-of-flight measurement at the detectors at $\pm 140 \mathrm{~cm}$.

The luminosity is measured over periods of $\sim 1$ minute called "luminosity blocks"; the variation of the instantaneous luminosity during this short time can be neglected.

The luminosity is calculated in the following way [61]. Expressed as

$$
\mathcal{L}=\frac{R}{\epsilon \sigma_{\text {inel }}}
$$

where $R$ is the event rate, $\epsilon$ is the efficiency (including the geometric acceptance) of the LM and $\sigma_{\text {inel }}$ is the inelastic cross-section as measured by other experiments. Since the LM can only determine whether at least one interaction occurred in a beam crossing, the average number of interactions per crossing is determined by counting the fraction of empty crossings instead, and assuming a Poisson distribution for the number of interactions in a given crossing. The inelastic cross-section used by $\mathrm{D} \varnothing$ and $\mathrm{CDF}$ is $60.7 \pm 2.4 \mathrm{mb}[62]$.

\subsubsection{Central Tracking System}

The central tracking system measures the momentum, direction, and the sign of the electric charge for charged particles produced in a collision. A solenoid provides a nearly uniform 2 Tesla magnetic field parallel to the beam axis. Charged particles leave a pattern of localized charge deposits called "hits" in the layers of the tracking detectors, and these hits are used to reconstruct a curved trajectory called a "track". The curvature of the track is proportional to the electric charge divided by the transverse momentum.

The central tracking system consists of the silicon microstrip tracker (SMT), the central fiber tracker (CFT), the solenoid, and the forward and central pre-showers. A cross section of the central tracking region is shown in figure 3.7 .

\footnotetext{
${ }^{1}$ In some usages, an "event" may instead refer to the data of one hard scatter, with a bunch crossing having multiple "events".

${ }^{2}$ The luminosity monitors are also used for selecting diffractive events.
} 


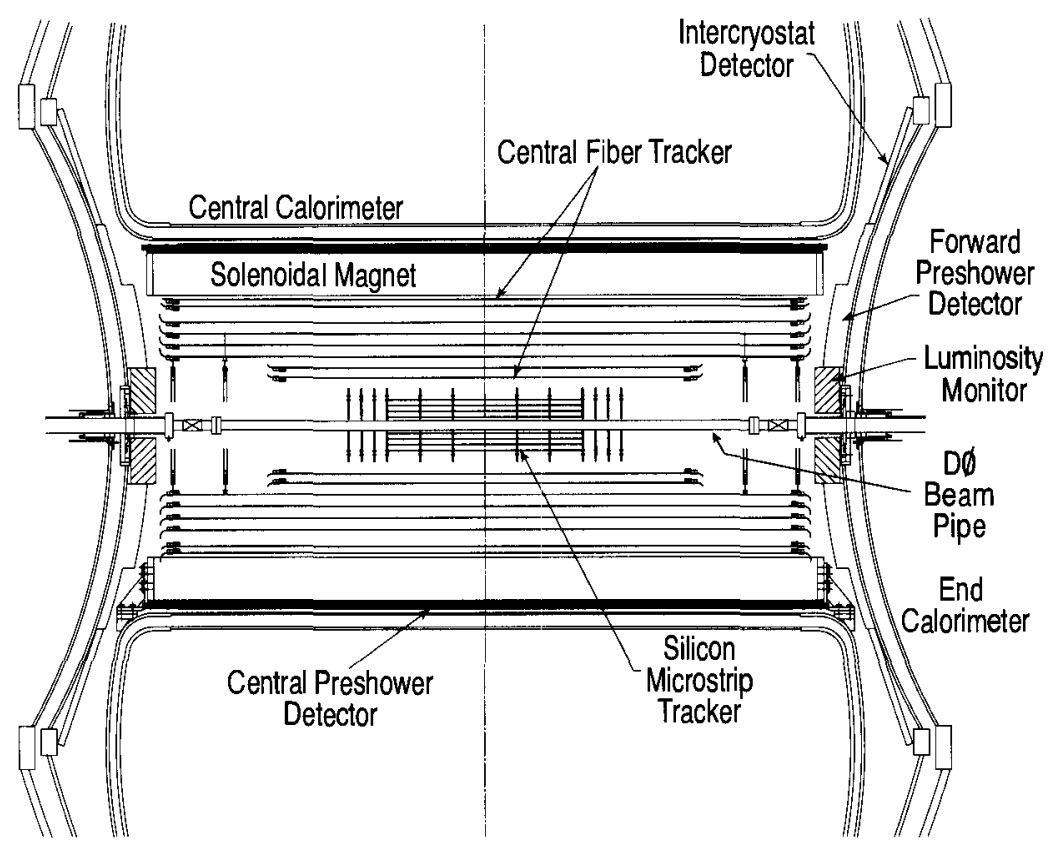

Figure 3.7: Cross section of the central tracking region in the $\mathrm{D} \varnothing$ detector.

The combined transverse momentum resolution is about $\left(2+0.15 p_{T}\right) \%$, with $p_{T}$ in $\mathrm{GeV}$. The impact parameter resolution in the $r-\phi$ plane is better than $15 \mu \mathrm{m}$ for tracks with a $p_{T}>10 \mathrm{GeV} / c($ at $\eta=0)$.

\section{Silicon microstrip tracker}

The silicon microstrip tracker (SMT) [63] is a silicon detector which uses reverse-biased $p-n$ junctions to create a depletion zone in the silicon, with an electric field across. When a particle passes through the semiconductor material, electrons and holes are created which are quickly collected at the opposing electrodes. This provides a signal of the incidence of the particle, while the fact that the electrodes collecting the charges come in narrow strips ('microstrips') provide a spatial measurement.

The SMT consists of three modules: six barrels (in the $z$-direction) instrumenting the central detector along the $z$-axis, twelve "F-disks" inserted vertically along the barrels and four "H-disks" covering the forward $(|\eta| \geq 2)$ regions. Barrels primarily measure the $r-\phi$ direction, while disks measure the $r-z$ and $r-\phi$ direction.

The length of the barrel region is $76 \mathrm{~cm}$, chosen because the length of the interaction region in $z$ is $\sigma=25 \mathrm{~cm}$. The SMT provides tracking and vertex reconstruction for almost the full $\eta$ range of the calorimeter and muon detectors. A 3-dimensional drawing of the SMT is shown in figure 3.8 .

Barrels and disks have $300 \mu \mathrm{m}$ n-type silicon wafers onto which narrow $p$-type strips are implanted, with a pitch of $50 \mu \mathrm{m}$. Some wafers have $n^{+}$-type strips on the reverse 


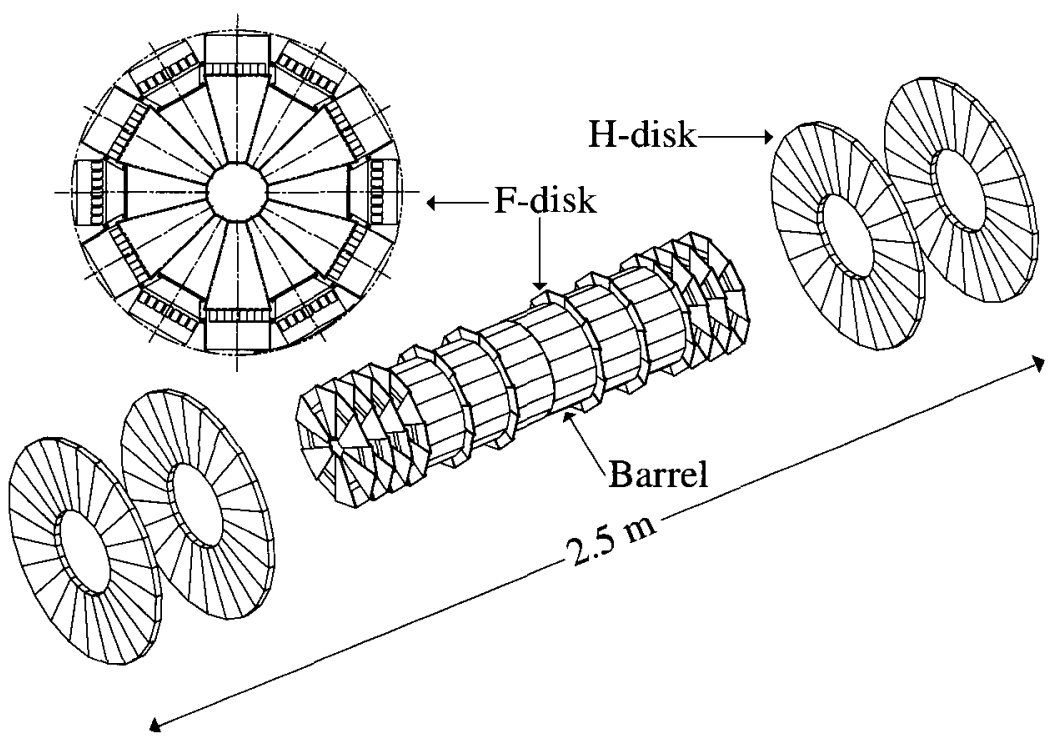

Figure 3.8: The silicon microstrip tracker.

side, stereoscopically aligned at $90^{\circ}$ or $2^{\circ}$ relative to the $p$-type strips to give a hit resolution along the $z$-axis of $35 \mu \mathrm{m}$ and $450 \mu \mathrm{m}$, respectively. For this reason, the double-sided barrels also allow $r-z$ measurements. The inner four barrels each have four double-sided layers, while the outer two barrels have 2 double-sided layers and 2 single-sided layers. The layers are spaced to achieve an axial position resolution of $\sim 20 \mu \mathrm{m}$. F-disks are made of twelve double-sided wedge detectors with strips aligned at stereo angles of $\pm 15^{\circ}$. H-disks are made of 24 single-sided wedge detectors glued back-to-back with strips aligned with stereo angles at $7.5^{\circ}$. The $\mathrm{H}$ disks provide coverage out to $|\eta|<3$.

There are almost 800,000 readout channels to the SMT. The signal to noise ratio is between $12: 1-18: 1$, depending on the detector type.

\section{Central Fiber Tracker}

Surrounding the SMT is the CFT [64], which provides tracking in the region $|\eta|<1.7$. It consists of scintillating fibers on eight concentric cylinders from 20 to $52 \mathrm{~cm}$ from the beam pipe. The two innermost cylinders are $1.66 \mathrm{~m}$ long, while the outer six cylinders are $2.52 \mathrm{~m}$ long. The CFT has 77,000 readout channels.

The fibers are arranged in overlapping layers of doublets of fibers. Each doublet consists of two layers that overlap by half a fiber width. This gives a track cluster resolution of $100 \mu \mathrm{m}$ per doublet layer. Each of the 8 cylinders has two doublet layers, with the outer doublet aligned at a stereo angle of $\pm 3^{\circ}$.

Charged particles passing through scintillating fibers cause the emission of light at $340 \mathrm{~nm}$ through rapid fluorescence decay. To increase the mean free path length of 
the resulting photons, fibers contain wave-shifting dye which absorb light at $340 \mathrm{~nm}$ and re-emit light at $530 \mathrm{~nm}$. This increases the propagation length to $4 \mathrm{~m}$, which is more than enough to travel to the end of the scintillating fiber. One end of the fiber is optically connected to a clear fiber waveguide, which carries the light to visible light photon counters (VLPCs) where photons are converted to electronic pulses. The other end of the scintillating fiber is coated with aluminum to reflect the light to the other end of the fiber.

The operation of VLPCs is based on the phenomenon of impurity band conduction, occurring when a semiconductor is heavily doped with shallow donors or acceptors. The impurity atoms are then so close together that the electrical transport may occur by charges hopping from impurity site to impurity site.

The VLPCs reside in cassettes partly placed in a liquid Helium cryostat located directly beneath the $\mathrm{D} \varnothing$ detector and operate at a temperature of $9 \mathrm{~K}$. The VLPCs have a fast response time, a quantum efficiency of greater than $75 \%$ and a high gain of $22,000-65,000$. They are capable of detecting single photons: the doublet hit efficiency is over $99.0 \%$ for cosmic muon tracks, with an average light yield of 8 photo-electrons per MIP (minimally ionizing particle).

One important feature of the CFT is its fast speed. Signals from the axial doublets are used for Level 1 triggering in the central track trigger (L1CTT). The L1CTT also sends the tracks it finds to the L1MUO system for matching to muon candidates and the L2STT for finding tracks using the SMT.

\section{Solenoid}

Momentum and charge sign measurements improved significantly in Run II with the addition of a highly uniform $2 \mathrm{~T}$ axial magnetic field [65]. This is maintained with a super-conducting solenoid surrounding the tracking region, fitting inside the central bore of the calorimeter. The solenoid is $2.73 \mathrm{~m}$ in length and $1.42 \mathrm{~m}$ in diameter. This magnetic field is maintained with a large $4825 \mathrm{~A}$ current. The solenoid is constructed of two types of super-conducting high-purity aluminum stabilized multi-filamentary $\mathrm{Cu}-\mathrm{NbTi}$ Rutherford cable maintained at $4.7 \mathrm{~K}$ in a cryostat.

\section{Preshower}

To compensate for the material in front of the calorimeters (i.e., the tracker, solenoid and supports), preshower detectors were installed outside of the central tracking system. Their purpose is to aid electron and photon identification, by providing both extra tracking to match tracks with calorimeter showers and an energy measurement.

Three layers of fibers make up the central pre-shower (CPS), with one layer parallel to the beam and the other two arranged at stereo angles of $\pm 20^{\circ}$. The forward preshower (FPS) covers the range $1.4<|\eta|<2.5$ and is mounted on the outer surface of the end calorimeter cryostat. It has two layers of scintillators at opposing stereo angles of $22^{\circ}$, a layer of lead with a thickness equivalent to 2 radiation lengths, and another two layers of scintillators with the same stereo angles. 


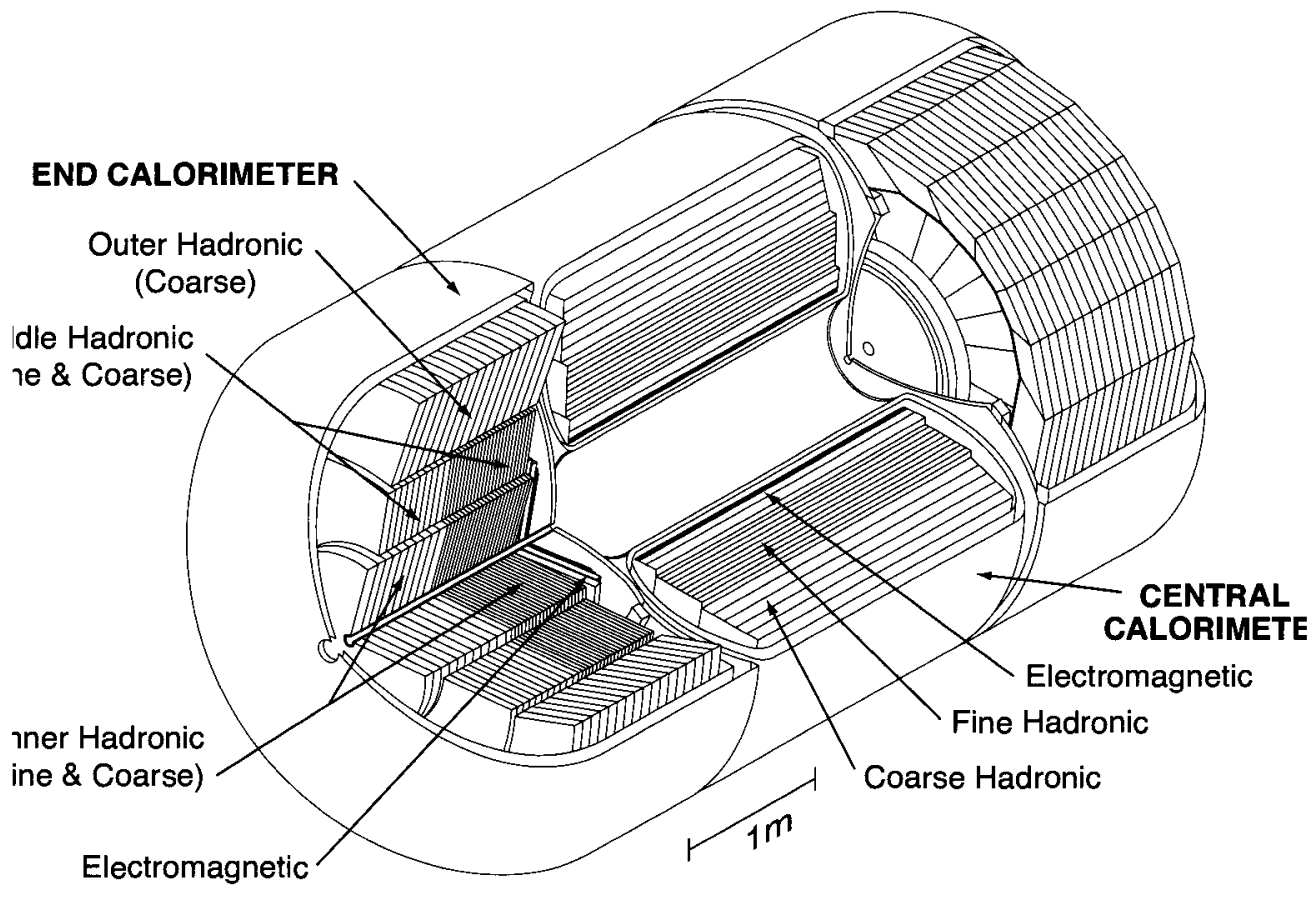

Figure 3.9: Cut-out view of the $\mathrm{D} \varnothing$ calorimeter.

\subsubsection{Calorimeter}

The calorimeter (figure 3.9) measures the energy from showers produced by particles that are absorbed in the calorimeter. The calorimeter [66] measures the energy, and aids with the identification primarily of electrons, photons, taus, and hadronic particles, all of which shower in the calorimeter. Muons with energies between 5 and $100 \mathrm{GeV}$ (see figure 3.10) are minimally ionizing particles and typically deposit an energy of $2-3 \mathrm{GeV}$ along their track in the calorimeter. Neutrinos typically do not interact with the $\mathrm{D} \varnothing$ detector at all.

In the calorimeter, electrons and photons produce electromagnetic showers; due to interactions with matter, electrons emit photons in a process called Bremsstrahlung, and photons in turn produce electron-positron pairs. The electromagnetic shower reaches a maximum when the average electron energy approaches a critical energy below which electrons lose more energy by ionization than by bremsstrahlung, and when the photons no longer have enough energy for pair production. After the shower maximum, the shower decays exponentially.

The thickness of detector materials is usually expressed in terms of the radiation length $X_{0}$, which is the length for an electron to have its energy reduced by a factor of $1 / e$ through radiation loss; the mean path photons travel before pair-producing electrons is approximately $\frac{9}{7} X_{0}[67]$.

Hadronic particles and jets also shower when interacting with matter, though the 


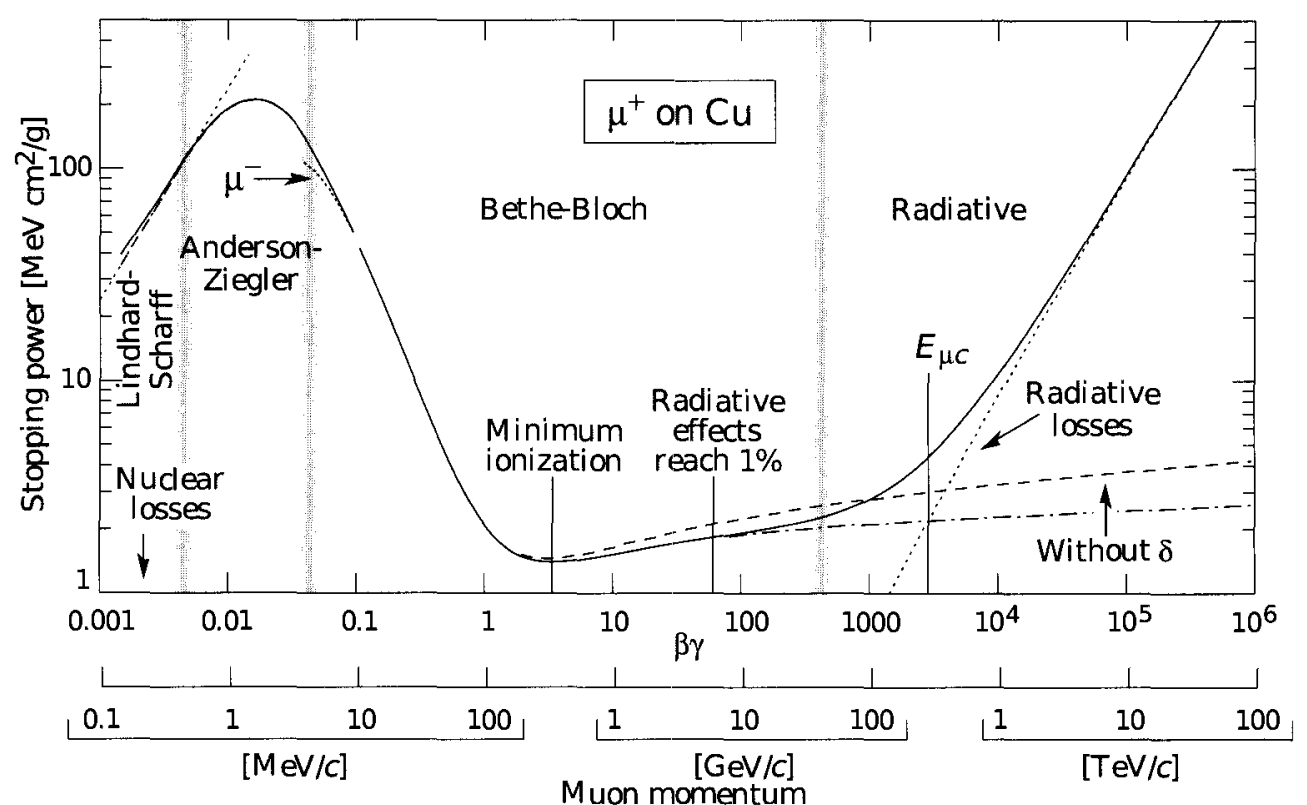

Figure 3.10: Stopping power $\left(=-\left\langle\frac{\mathrm{d} E}{\mathrm{~d} x}\right\rangle\right)$ as a function of muon momentum for muons in copper [16].

shower progression is more complex [68]. The interaction length $\left(\lambda_{A}\right)$ or mean path between interactions that are not elastic or diffractive, provides a scale for the shower development. Hadron showers develop over a longer distance than electromagnetic showers, especially for high atomic number materials. The D $\varnothing$ calorimeter is a compensating calorimeter, for which the hadron to electromagnetic signal ratio is close to one.

The $\mathrm{D} \varnothing$ calorimeter is divided into a central calorimeter (CC), which covers up to $|\eta|<1$, and two end calorimeters (EC) extending all the way to $|\eta| \sim 4$. The section of the calorimeter closest to the interaction region, known as the electromagnetic $(\mathrm{EM})$ section, is designed to measure electromagnetic particles. The outer two sections are the fine hadronic $(\mathrm{FH})$ and coarse hadronic $(\mathrm{CH})$ sections.

The $\mathrm{D} \varnothing$ calorimeter consists of alternating layers of absorber plates (see figure 3.9), mainly made of uranium, and readout cells, with an active medium of liquid argon in the gaps between them. To achieve a similar energy response in each section of the calorimeter, different materials are used for absorber plates. The EM section uses 3-4 $\mathrm{mm}$ thick depleted uranium plates with a total radiation length of about $20 X_{0}$. The FH section uses $6 \mathrm{~mm}$ thick plates made of a uranium-niobium alloy. The $\mathrm{CH}$ section uses $46.5 \mathrm{~mm}$ copper plates in the $\mathrm{CC}$ and stainless steel plates of the same size in the EC. The calorimeter is located within a cryostat that maintains the temperature at $80 \mathrm{~K}$. The total thickness of the hadronic calorimeter is about six nuclear interaction lengths $\lambda_{A}$ in the CC and up to nine in the EC.

The radiation length and the interaction length of the absorber plates is short, so 
the particles are stopped in a relatively short distance. The liquid argon in between is ionized by charged particles passing through it and the charges are collected by a readout cell.

Readout cells consists of copper readout pads covered in a resistive epoxy. An electric field is created between the absorber plate and the readout pad by holding the absorber plate at ground and holding the resistive surface of the pad at $\sim 1.6 \mathrm{keV}$. The gap between absorber and readout is $2.3 \mathrm{~mm}$, and the maximum time for electrons to drift across this gap is approximately $450 \mathrm{~ns}$; therefore the calorimeter readout is slow compared to the inner detectors.

The segmentation of the readout layers into cells means that the location of the showers can be determined as well as their energy. The coverage for a readout cell in the CC is roughly $\Delta \eta \times \Delta \phi=0.1 \times 0.1$, except in the third layer of the EM section (where the shower maximum for electrons and photons is expected to occur) which has cells with a coverage area of $0.05 \times 0.05$ for enhanced precision.

The pseudo-rapidity region $0.8<|\eta|<1.4$ is not well covered due to gaps between the $\mathrm{EC}$ and $\mathrm{CC}$ sections of the calorimeter. To improve the energy resolution in this region, a single-layered scintillation detector is installed in the gap between the EC and CC cryostats. This detector is called the inter-cryostat detector (ICD). The segmentation is the same as in the liquid argon calorimeter.

\subsubsection{Muon system}

Muons deposit very little energy in the calorimeter, making them difficult to detect and identify there. A dedicated muon system is used, therefore, to identify muons, provide approximate locations, momenta, and charges of these muons, and allow for fast triggers based on the presence of high energy muons likely to have come from the interaction region. The muon system [69] is placed outside the calorimeter because all particles predicted by the standard model other than muons (except neutrinos) are typically absorbed in the D $\varnothing$ calorimeter. Muons with energies up to a few hundred $\mathrm{GeV}$ interact primarily through ionization rather than bremsstrahlung. Figure 3.10 shows the energy loss for muons (in copper). A muon needs about $1.6 \mathrm{GeV}$ of energy to exit the calorimeter [69].

The muon system was designed to detect the energy loss of muons as they pass through the detector. It is a spectrometer consisting of drift tubes and scintillators. The central and forward systems each have dedicated 1.9 Tesla toroidal magnets. The purpose of the magnets is to to measure the momentum of muon locally. They run at a lower current than in Run $\mathbf{I}$ as a cost-saving measure, because the momentum of muons is primarily measured in the central tracker now. Together with the iron in the shielding assemblies, the toroid also returns the magnetic field lines of the solenoid magnet. The average energy loss of a muon across the toroidal magnet is $1.7 \mathrm{GeV}$.

The central region of the muon system covers up to $|\eta|<1$, while the forward regions cover $1<|\eta|<2$. Both regions have an A-layer of drift tubes and scintillators placed outside the calorimeter and inside the muon system toroids. Both regions also have B- and C-layer drift tubes and scintillators located outside the toroids. Figure 3.11 shows the arrangement of the wire chambers and scintillators. Note that the bottom 


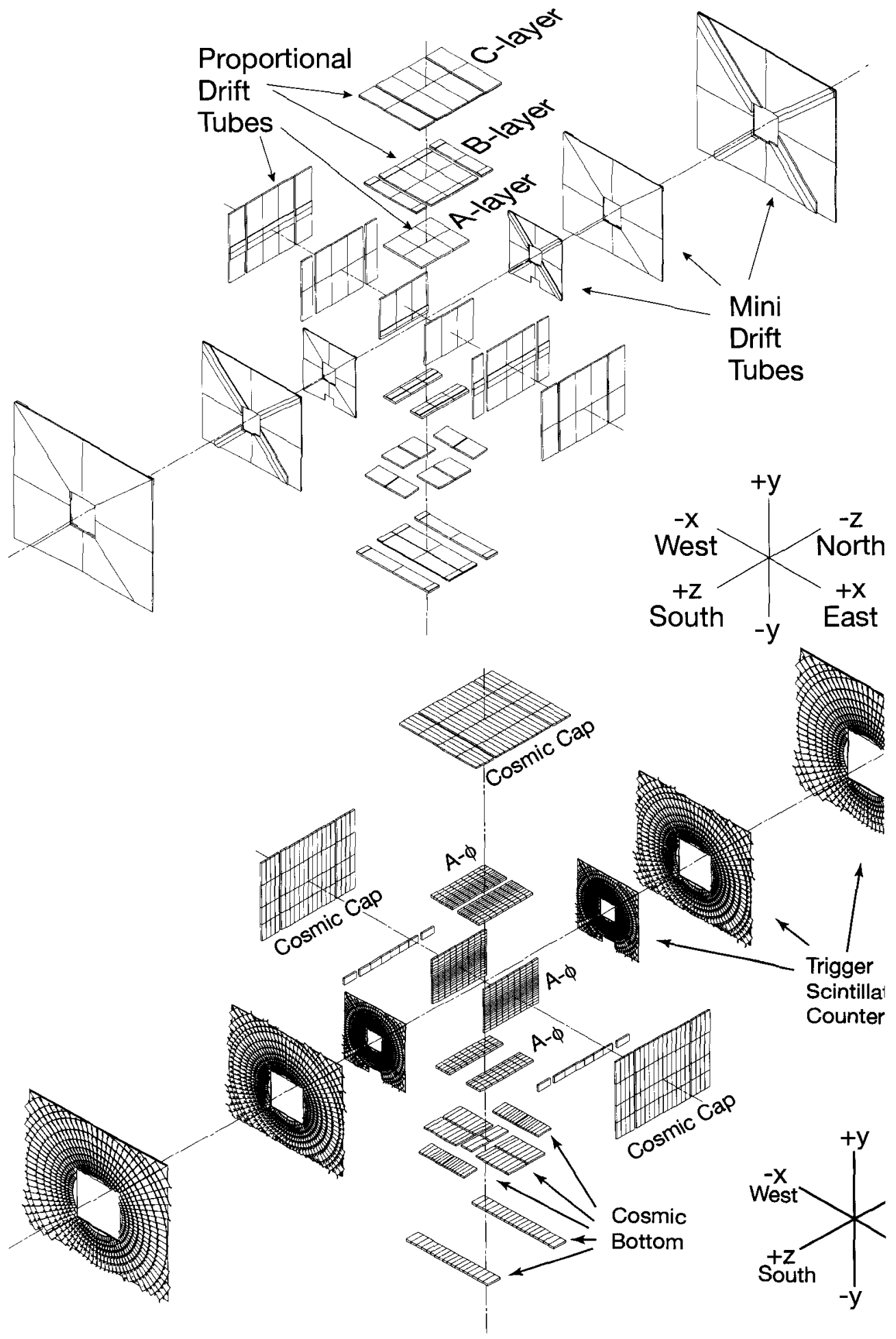

Figure 3.11: Exploded view of the muon system wire chambers (top) and scintillators (bottom) [59]. 
of the detector is not fully instrumented, because that is where the support structure for the detector is located.

The purpose of the drift tubes is to accurately measure the position of the muons (and for triggering). Each drift tube has a wire running lengthwise down the center, held at a positive voltage with respect to the tube walls. Multiple drift tubes are arranged in larger chambers that contain one or more layers of tubes arranged side by side. The chamber contains a freely circulating gas, in which electrons are formed through ionization as a muon passes through it. These electrons move toward the sensing wires, causing further ionizations, and the resulting avalanche of electrons is detected by the electronics connected to the wire.

The purpose of the scintillators is to have a fast readout for accurate timing, rejecting cosmics, matching wire chamber hits with bunch crossings, and for triggering as well. As charged particles pass through, the scintillating material in these detectors emit photons, which are carried by wavelength-shifting materials to photo multiplier tubes for detection. The PMTs send an electrical pulse to the muon scintillator front-end boards located in crates inside the detector, where it is used to determine the time that the muon passed through the scintillator. The signal is calibrated to be registered, on average, at $0 \mathrm{~ns}$ for a hit coming from the $p \bar{p}$ collision.

The central scintillators are rectangular in shape, while the forward scintillators are trapezoidal in shape. Both have a $\phi$ segmentation of $\sim 4.5^{\circ}$ and a time resolution of $\sim 2$ ns.

The drift tubes in the central region are housed in proportional drift tube (PDT) chambers, (see figure 3.12) while those in the forward regions have Mini Drift Tube (MDT) chambers. The B and C layers have three rows, or "decks", of cells each, while the A layer has four decks, except along the bottom of the detector, where it has three decks. Approximately 55\% of the central detector is covered by three layers of PDTs, and $90 \%$ by at least two layers. The wires are arranged parallel to the toroidal magnetic field, which is in the y direction for the PDTs on the sides, and in the $\mathrm{x}$ direction for the top and bottom PDTs.

The PDTs have a drift cell width of $10.1 \mathrm{~cm}$ and a maximum drift time of $500 \mathrm{~ns}$ while the MDTs have a cross section of only $9.4 \times 9.4 \mathrm{~mm}^{2}$ and a maximum drift time of 60 ns. The PDTs use $\mathrm{Ar}(80 \%), \mathrm{CH}_{4}(10 \%)$ and $\mathrm{CF}_{4}(10 \%)$ gas while the MDTs use only the latter two $(90 \%, 10 \%$ respectively).

For each muon hit, the drift chambers provide several measurements: first, the drift time to the anode wire, which gives the axial distance of the muon hit from the wire. Secondly, because neighboring cells are connected, the difference in the arrival time of the hit between a hit cell and a neighboring cell can be measured; this gives the approximate lengthwise position on the wire. The single cell hit resolution perpendicular to the wire is about $1 \mathrm{~mm}$. The central muon system has a momentum resolution of $\sigma(p) / p=0.36(p-3.1) / p \oplus 0.03 p$ (where the momentum $p$ is in $\mathrm{GeV}$ ) [70] and the forward muon system of $\sim 20 \%$.

The performance of the muon system is stable over time to about $1 \%$. Of the installed drift tubes, $98.6 \%$ (central) and $99.7 \%$ (forward) are active, for scintillators this is $99.8 \%$ and $99.9 \%$ respectively 

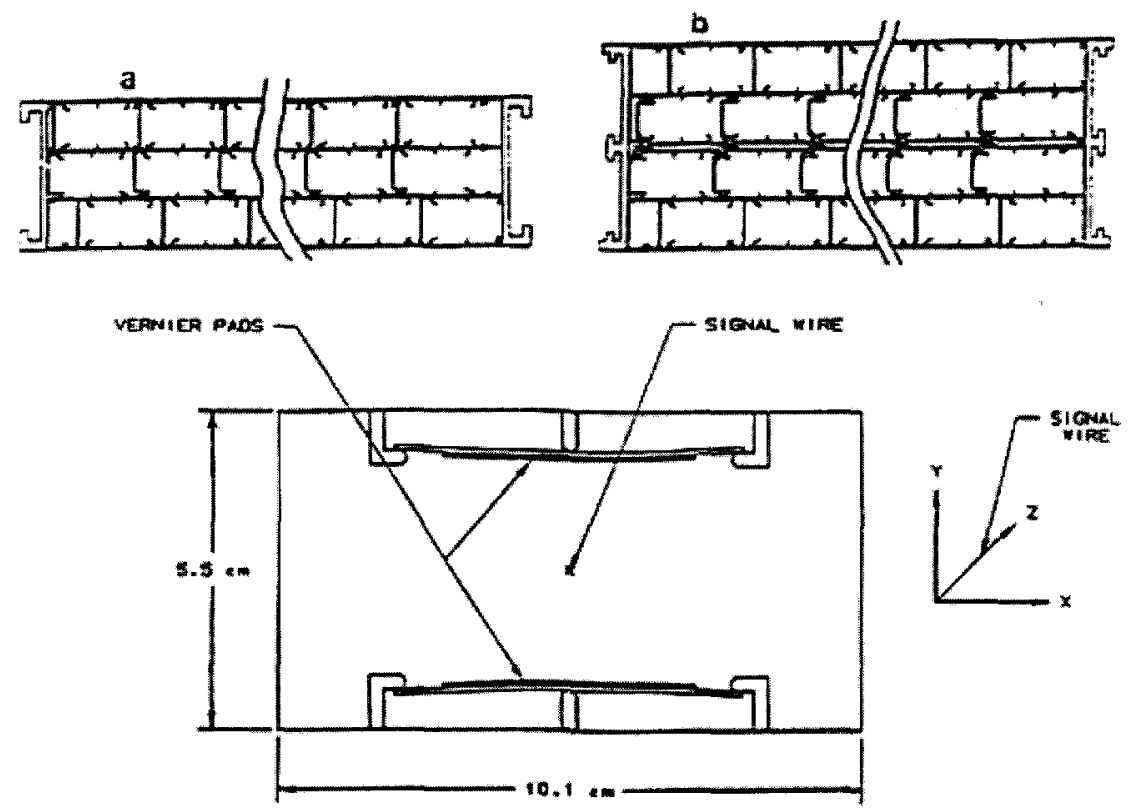

Figure 3.12: Central muon system PDTs. (a) shows the end view of the 3-deck (B and C-layer) extrusion. (b) shows the end view of the 4-deck (A-layer) extrusion. The figure below shows the end view of a single cell, including the cathode pad.

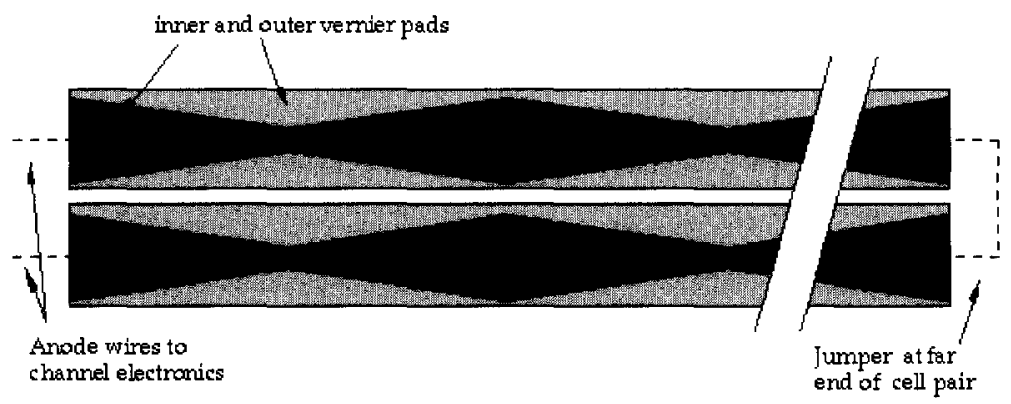

Figure 3.13: Central muon PDT readout pads viewed from above. 
Additionally, in some cases the charge deposition on the inner and outer vernier pads can be measured: on two sides of each PDT cell are cathode pads, which collect the ions. Each cell side has two tapered pads, arranged as an inner and outer pad. The inner and outer pads on each side of the cell are joined, giving a total of two pads per cell. Because the pads are tapered, the ratio of the charge collected on each pad contains information on the position at which the charge was created, i.e., in the $z$-direction. The geometry of the pads shows a periodic structure with a wavelength of approximately $61 \mathrm{~cm}$ (see figure 3.13). In the A-layer, all of the PDT cells have instrumented pads, whereas in the B- and C-layers only $10 \%$ of the pads are instrumented.

More information on the muon reconstruction is presented in section 5.2.

\section{Muon triggers}

The analysis presented in this thesis looks at muons, and the selected events are required to have triggered one of the many single- or dimuon triggers available (see section 5.1.3). Muons are triggered at Level 1, either by combinations of raw PDT, MDT and scintillator hits in a specified number of layers and regions, or by matching scintillator hits to tracks reconstructed by the central track trigger at level 1 (L1CTT). Triggers including a match to a L1CTT track usually involve a cut on the transverse momentum of the track.

At level 2, muon triggers repeat the $\mathrm{L} 1$ reconstruction with more precision, now using MDT and PDT hits that are fully reconstructed using the drift time and wire $\phi$ position, to reconstruct 3-dimensional tracks separately in each layer of the muon system, then combine them into muon tracks. 


\section{Chapter 4}

\section{Simulation}

A simulation was run in order to estimate the acceptances and efficiencies. The expected particle content, kinematic distributions and reconstructed quantities are calculated, both for Standard Model processes that can contribute significantly to the selected sample (background) and for the new physics processes that are searched for (signal). The simulation factorizes into a prediction of the elementary particle interactions from the Standard Model or an extension of it, and a description of the detector acceptance and efficiency for the particle content of a given event.

Because the calculations of the final probability densities involve integrals over a very large phase space, they can not possibly or practically be done exactly. Instead, the answers are estimated by randomly sampling a large number of simulated collision events from the production probability distributions in a calculative method called "Monte Carlo". This process is factorized analogously to the way the physical process factorizes in the real world.

Generator First, the interactions are randomly produced according to the predicted scattering cross sections. Initial- and final state radiation and parton showering are simulated separately, analogously to the way these processes factor out in QCD (see section 2.2). These calculations are together called the 'event generation'; here, PYTHIA v6.3 was used [71].

In principle, for each generated event, PYTHIA calculates a possible total set of outgoing particles that are produced in a beam crossing, given a specified number of hard subprocesses and final state particles, plus the cross section for that event.

This includes the initial- and final state radiative corrections and parton showers as well as the effects of "underlying" events, which are the subprocesses involving the hadron remnants not involved in the hard scattering subprocess.

For each sample, the hard scatter interaction subprocess cross section was calculated to leading order (LO) in the coupling constants and convoluted with the CTEQ6L1 parton distribution functions (also calculated to $\mathrm{LO}$ ) which are compiled by the CTEQ collaboration[72, 73]. 
Detector Simulation Secondly, the detector response is simulated with a detailed detector material simulation, called dogstar [74](the version from the $\mathrm{p} 17$ release was used), which uses GEANT3 [75], in combination with a simulation of the electronics called dosim [76] .

To estimate the effects of multiple interactions and pile-up, for each event the PYTHIA output is overlayed with zero-bias (i.e., random crossing) raw (detector level) data, which was taken in special runs. The combined output is then processed by the reconstruction algorithm ( $\mathrm{p} 17$ version of doreco), in the same way as the data is.

\subsection{Monte Carlo samples}

To estimate the expected background, all Standard Model physics processes expected to contribute non-negligibly to the pre-selected data sample were simulated. The samples include processes where jets are produced due to initial state QCD radiation (indicated by (+Jets)).

\subsubsection{Background samples}

The following samples were used:

- $\gamma^{\star} / Z \rightarrow \mu^{+} \mu^{-}$(+Jets) with $M_{\gamma^{*} / Z}>15 \mathrm{GeV} / c^{2}$

where $M_{\gamma^{*} / Z}=\sqrt{\hat{s}}$ of the Drell-Yan hard interaction. This simulates an irreducible background.

- $\gamma^{\star} / Z \rightarrow \tau^{+} \tau^{-}$(+Jets) with $M_{\gamma^{\star} / Z}>15 \mathrm{GeV} / c^{2}$

Here the main contribution comes from events where the tau and anti-tau decay as $\tau^{+} \rightarrow \bar{\nu}_{\tau} \nu_{\mu} \mu^{+}$and $\tau^{-} \rightarrow \nu_{\tau} \bar{\nu}_{\mu} \mu^{-}$(each with a branching fraction of $\sim 17 \%$ ) respectively.

- $W \rightarrow \mu \nu(+$ Jets $)$

Here, a second (anti-) muon can come from a heavy quark jet.

- $t \bar{t} \rightarrow$ inclusive

Muons are produced either in bottom quark jets or in the decay of a $W$ boson.

Table 4.1 lists the total number of generated events after the removal of duplicate events (which can occur through mistakes in the production process) and of events that were overlaid with zero-bias events which did not pass data quality requirements (see section 5.1.1), as well as the (leading-order) PYTHIA cross-section for all samples. 


\begin{tabular}{|ccccc|}
\hline Production & Decay & & \# events & LO $\sigma \times$ B.R. $(\mathrm{pb})$ \\
\hline$\gamma^{\star} / Z$ & $\mu^{+} \mu^{-}+X$ & $15<M_{\gamma^{*} / Z}<60$ & $1.94 \mathrm{M}$ & 363 \\
& & $60<M_{\gamma^{\star} / Z}<130$ & $2.80 \mathrm{M}$ & 180 \\
& & $130<M_{\gamma^{\star} / Z}<250$ & $384 \mathrm{k}$ & 1.35 \\
& & $250<M_{\gamma^{\star} / Z}<500$ & $953 \mathrm{k}$ & 0.114 \\
& & $500<M_{\gamma^{\star} / Z}$ & $47.4 \mathrm{k}$ & $4.57 \times 10^{-3}$ \\
& & $15<M_{\gamma^{\star} / Z}<60$ & $388 \mathrm{k}$ & 363 \\
$\gamma^{\star} / Z$ & $\tau^{+} \tau^{-}+X$ & $60<M_{\gamma^{\star} / Z}<130$ & $927 \mathrm{k}$ & 180 \\
& & $130<M_{\gamma^{\star} / Z}<250$ & $380 \mathrm{k}$ & 1.35 \\
& & $250<M_{\gamma^{\star} / Z}<500$ & $94.9 \mathrm{k}$ & 0.114 \\
& & $500<M_{\gamma^{\star} / Z}$ & $54.3 \mathrm{k}$ & $4.57 \times 10^{-3}$ \\
& & & $3.04 \mathrm{M}$ & 1912 \\
$t \bar{t}$ & $\mu \nu+X$ & & $558 \mathrm{k}$ & 7.0 \\
\hline
\end{tabular}

Table 4.1: Generated background samples, production and decay channel(s), with the number of generated events after removing duplicates and leading order total crosssection times branching ratio for the given channel(s) (calculated with PYTHIA 6.3). $M_{\gamma^{\star} / Z}$ is given in $\mathrm{GeV} / c^{2}$.

\section{Other backgrounds}

Remaining physics processes that can contribute to the background and/or the $Z$ peak normalization are muons from cosmic events and muons from $\gamma^{\star} / Z \rightarrow b \bar{b}$ with muons originating from the bottom quark jets. Cosmic events are removed by a combination of tight cuts (see section 5.4.5). It will be argued that the remaining contribution from $\gamma^{\star} / Z \rightarrow b \bar{b}$ decays in the final selected samples is negligible (see section 5.4.3). Therefore these processes are not simulated.

\subsubsection{Signal samples}

\section{Standard Model-like $Z^{\prime}$ model}

To estimate the acceptance times selection efficiency for signal events and its dependence on the $Z^{\prime}$ mass parameter, about 10000 events were generated for each of 10 samples with the $Z^{\prime}$ mass taking values between 400 and $1000 \mathrm{GeV} / c^{2}$. Because interference effects between the $Z^{\prime}$ and the Standard Model $\gamma^{\star} / Z$ are negligible in this mass range (see section (2.3.3)), instead of generating the full $\gamma^{\star} / Z / Z^{\prime}$ signal, only the $Z^{\prime}$ resonance is generated and the signal plus Standard Model differential cross-section is simply taken as the direct sum of the $Z^{\prime}$ resonance plus the Standard Model $\gamma^{\star} / Z$ background. 


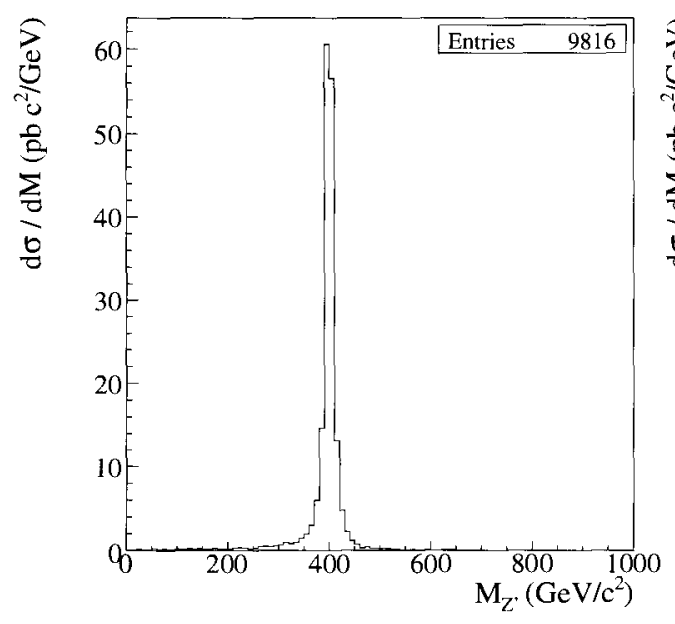

(a)

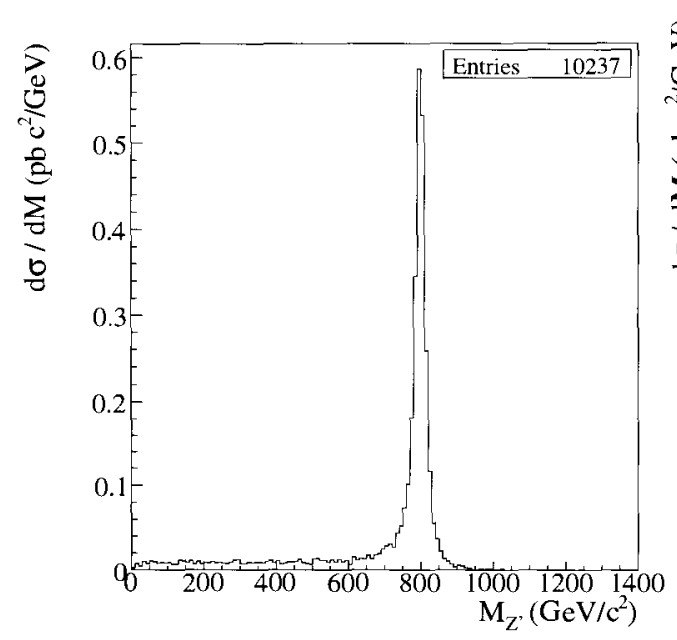

(c)

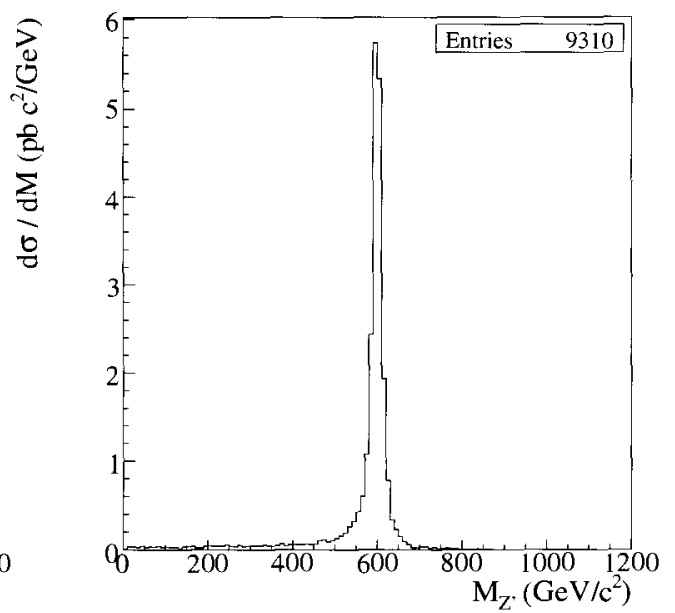

(b)

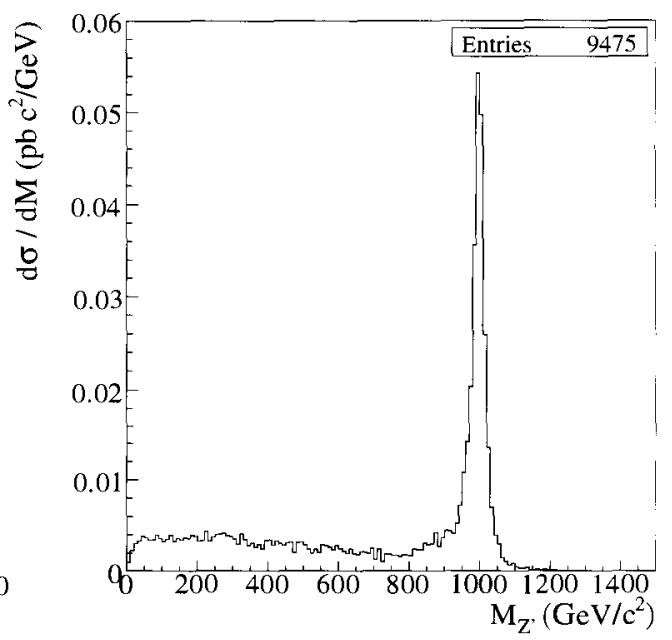

(d)

Figure 4.1: Generated boson mass distributions for the Standard Model-like $Z^{\prime}$, for $M_{Z^{\prime}}=400$ (a), 600 (b), 800 (c), and 1000 (d) $\mathrm{GeV} / \mathrm{c}^{2}$. 
For the Standard Model-like $Z^{\prime}$ model, the width of the $Z^{\prime}$ is that of a $Z$ boson with a mass $M_{Z^{\prime}}$ :

$$
\Gamma_{Z^{\prime}}=\left(\Gamma_{Z} / M_{Z} \cdot M_{Z^{\prime}}\right) \approx 0.027 \cdot M_{Z^{\prime}}\left(\mathrm{GeV} / c^{2}\right)
$$

which is negligible compared to the reconstructed mass resolution for $M_{Z^{\prime}} \gg M_{Z}$. Figures 4.1(a) to 4.1(d) show the generated differential cross-sections for a few $Z^{\prime}$ mass points.

While for two-particle initial states the $Z^{\prime}$ resonance, like the $Z$ resonance, would give a symmetric, Breit-Wigner line shape (if we ignore the effect of photon radiation), the effect of the parton distributions on the observable resonance shape is to relatively enhance the low-mass tail and suppress the high-mass tail (see, eg., [71, p. 99]). This effect becomes more pronounced the higher the $Z^{\prime}$ mass is, compounded by the increasing $Z^{\prime}$ width.

\section{Randall-Sundrum Model}

The same procedure was followed for the Randall-Sundrum model. Only the lightest massive resonance $G^{[1]}$ is simulated and searched for. This is conservative because the sensitivity to the full range of resonances $G^{[n]}$ would be higher. The difference is small because of the decreasing production cross section and increasing width, and the fact that for all mass points except $M_{G^{[1]}}=200 \mathrm{GeV} / c^{2}$, no upper mass cut is used (see 6.1.1). Moreover, existing searches put a limit on the lightest resonance as well. Several samples with different boson mass $M_{G^{[1]}}$ were produced, with $k / M_{\text {Planck }}=$ 0.01 fixed. Figures 4.2 show the generated boson mass distributions.

\subsection{Corrections and uncertainties}

\section{NNLO QCD K-factor}

A mass-dependent QCD K-factor, defined as

$$
\mathrm{K}_{\mathrm{NNLO}}\left(M_{\mathrm{DY}}\right)=\sigma_{\mathrm{NNLO}}(\mathrm{CTEQ} 6.1 \mathrm{M}) / \sigma_{\mathrm{LO}}(\mathrm{CTEQ} 6 \mathrm{~L} 1)\left(M_{\mathrm{DY}}\right)
$$

is applied to both the Standard Model $\gamma^{\star} / Z \rightarrow \mu^{+} \mu^{-}, \tau^{+} \tau^{-}$backgrounds and the $Z^{\prime}$ signal. Here, $\sigma_{(\mathrm{NN}) \mathrm{LO}}((\mathrm{N}) \mathrm{LO} \mathrm{pdf})$ is the (next to-next to) leading order Drell-Yan cross section [77] computed with a parton density function measured relative to a leading order (CTEQ6L1) or next to leading cross section (CTEQ6.1M). A linear interpolation is used to obtain a $\mathrm{K}$-factor with errors for continuous values of the Drell-Yan mass.

Errors on the (normalized) Drell-Yan production cross sections due to uncertainties in the parton distribution functions were calculated in [78] by adding in quadrature the variations of the (differential) cross-sections calculated with each of 40 CTEQ6.1M (normalized) error functions, following the prescription of [72]. The same prescription was followed to calculate the error on the signal acceptance; see section 6.2.2 for more 


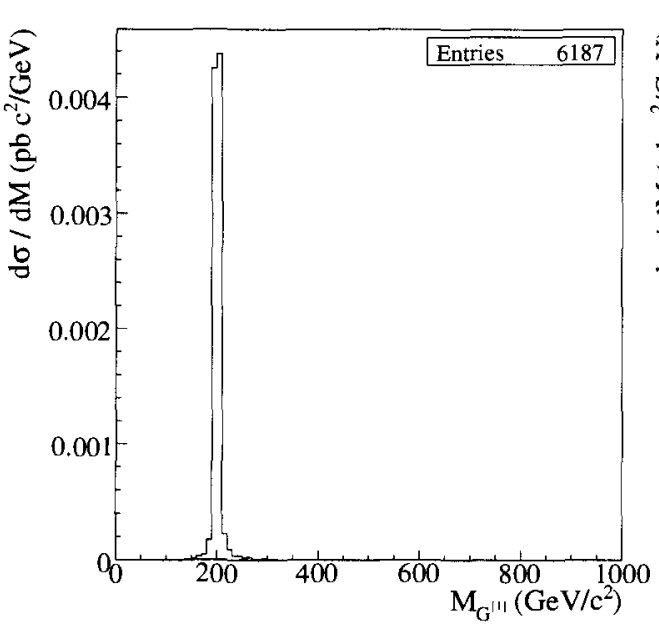

(a)

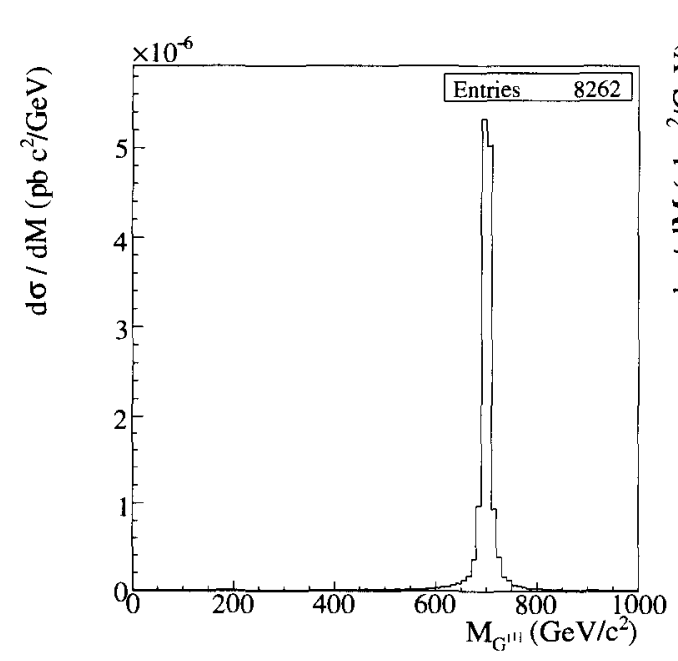

(c)

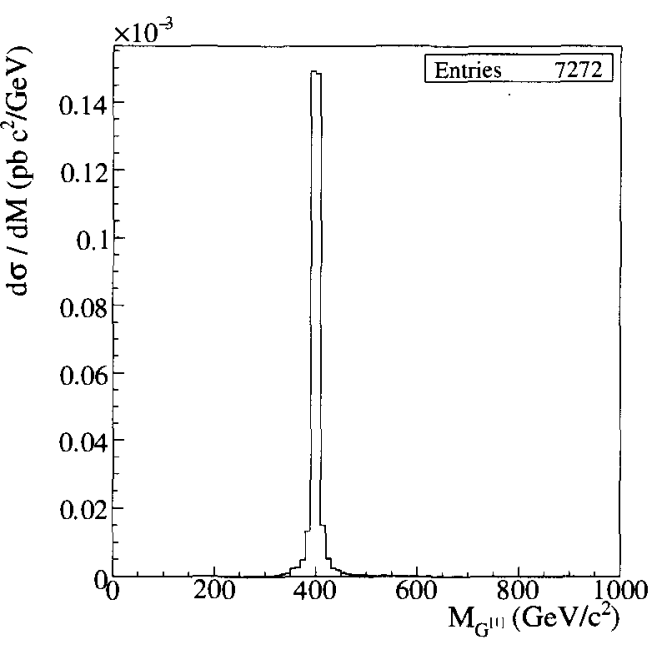

(b)

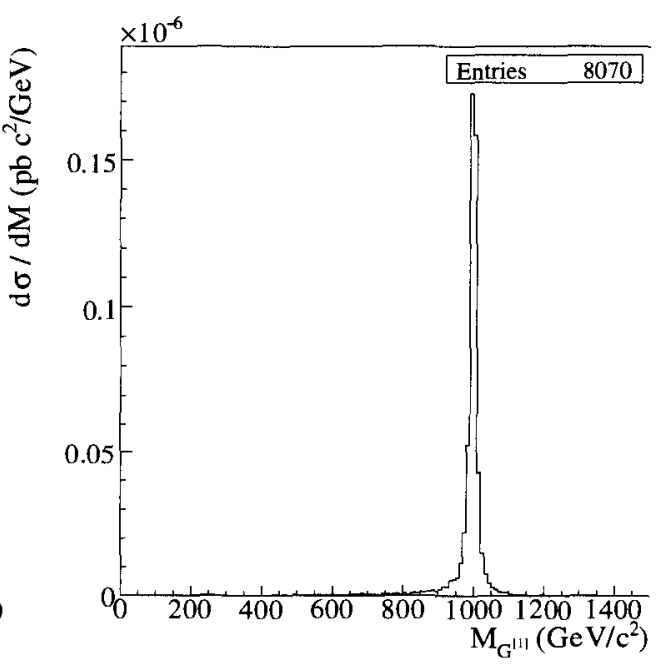

(d)

Figure 4.2: Generated mass distributions for the Randall-Sundrum model, for $M_{G^{[1]}}=$ 200 (a), 400 (b), 700 (c), and 1000 (d) $\mathrm{GeV} / c^{2}$. 


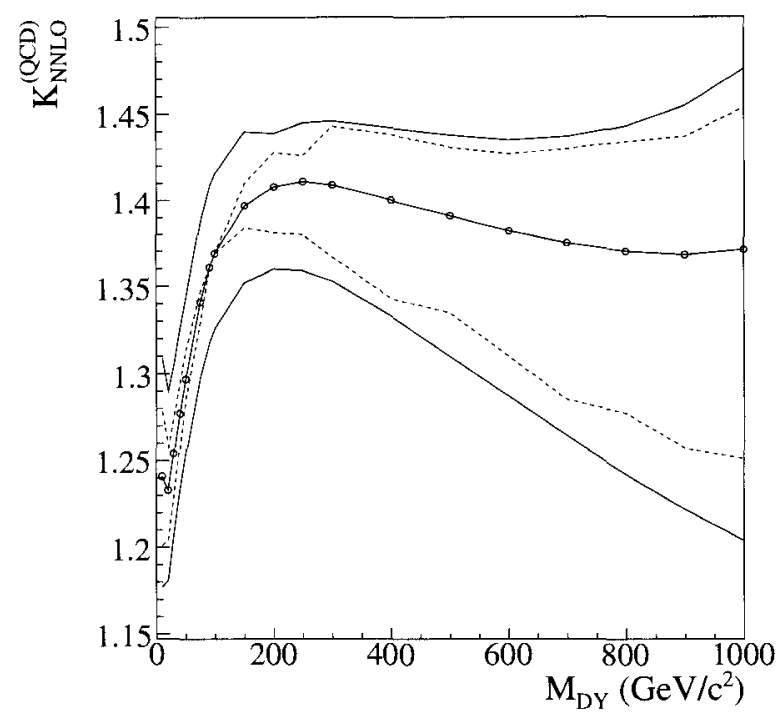

Figure 4.3: $K_{N N L O}$ as a function of the Drell-Yan mass. The solid error band represents the uncertainty on the NNLO cross section due to the PDF uncertainty; the dashed error band represents the same PDF uncertainty on the ratio of the cross section to the $Z$ peak cross section.

details. Figure 4.3 shows the K-factor and ituncertainty as a function of $M_{\gamma^{*}} / Z$. Because the Standard Model background is normalized to the $Z$ peak in data (see section 5.4.1), the quantity of interest there is the uncertainty on the NNLO cross section divided by the NNLO $Z$ cross section.

The PDF uncertainty on the NNLO $Z^{\prime}$ cross section is the same as the uncertainty on the NNLO $\gamma^{\star} / Z$ cross section.

The error on the NNLO $Z$ and $W^{ \pm}$cross section due to higher-order corrections was also estimated in [78] by simultaneously varying the factorization and renormalization scales between $0.5 M_{\gamma^{*} / Z}$ and $2 M_{\gamma^{*} / Z}$. They are on the order of $0.3 \%$ and $0.2 \%$, respectively.

\section{NLO EW corrections}

The full NNLO (QCD), NLO (EW) Drell-Yan cross section has not been calculated yet at the moment of writing. Instead, the $\mathcal{O}(\alpha)$ radiative corrections to the LO $q \bar{q}$ cross-section were calculated with the HORACE event generator [79]. Figures 4.4 show the resulting $K^{(E W)}$-factor, defined as

$$
K_{\mathrm{NLO}}^{(\mathrm{EW})}=\sigma_{\mathrm{NLO} \text { EW }, \text { LO QCD }}(\mathrm{CTEQ} 6 \mathrm{~L} 1) / \sigma_{\mathrm{LO} \text { EW }, \text { LO QCD }}(\mathrm{CTEQ} 6 \mathrm{~L} 1)
$$

calculated with the CTEQ6L1 LO PDF (hence not including photon-induced corrections) with the following cuts on the muons applied: 

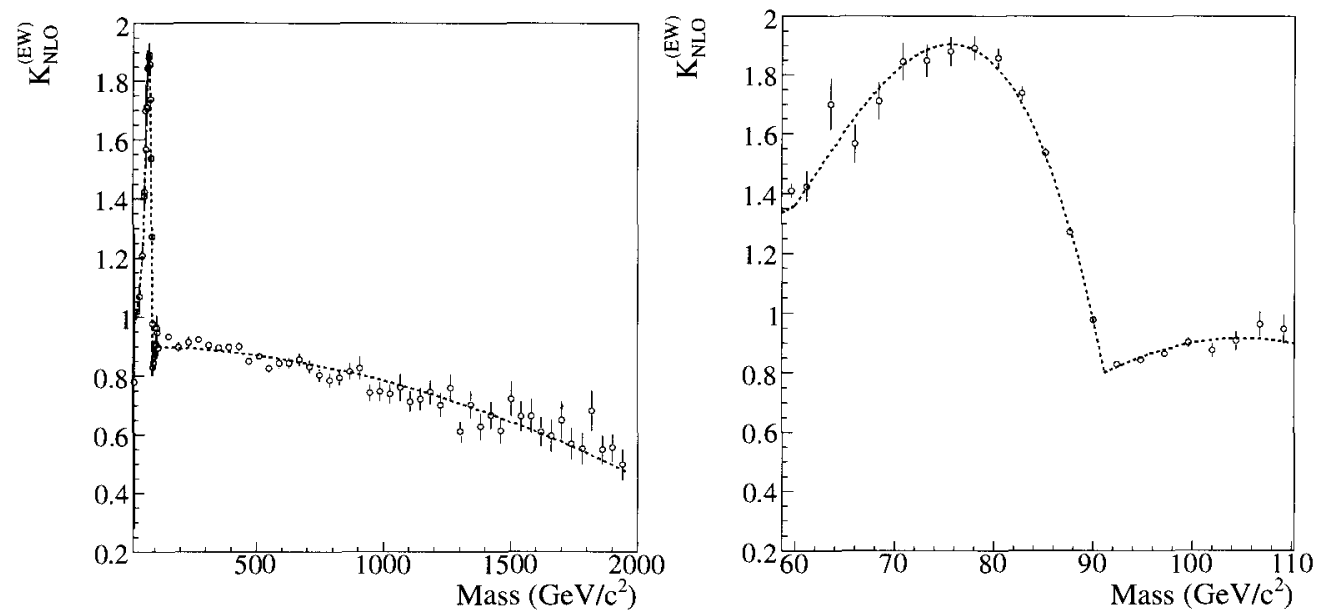

Figure 4.4: Relative size of the $\mathcal{O}(\alpha)$ radiative corrections to the Born level (LO EW) Drell-Yan cross-section, omitting photon-induced corrections, calculated using the HORACE event generator. (a) shows the full range, (b) the interval around the $Z$ mass. Also shown is an ad-hoc piecewise continuous polynomial fit.

- $|\eta|<2$

- $p_{T}>10 \mathrm{GeV} / \mathrm{c}$

The inclusion of this correction to the Drell-Yan background (and similar ones for the signal processes) influence the analysis by the normalization to the $Z$ peak, the calculation of the expected background ${ }^{1}$ and the signal acceptances and cross-sections. However, in the following, the electroweak K-factor is not applied, because

- it is a priori not obvious whether indeed

$K_{\mathrm{NLO}}^{(\mathrm{EW})} \cdot K_{\mathrm{NNLO}}^{(\mathrm{QCD})} \cdot \sigma_{\mathrm{LO} \text { EW, LO QCD }}(\mathrm{CTEQ} 6 \mathrm{~L} 1) \approx \sigma_{\mathrm{NLO}} \mathrm{EW}, \mathrm{NNLO} \mathrm{QCD}(\mathrm{CTEQ} 6.1 \mathrm{M})$

- the uncertainties on this quantity were not calculated,

- the correct $K_{\mathrm{NLO}}^{(\mathrm{EW})}$ for the $Z^{\prime}$ models was not calculated.

Instead, the results with the NLO EW correction factor applied are presented in appendix $\mathrm{A}$, as an indication of the possible size of this effect. The results presented in the main body, without the NLO EW correction, can be directly compared to earlier analyses which also do not apply this correction. 


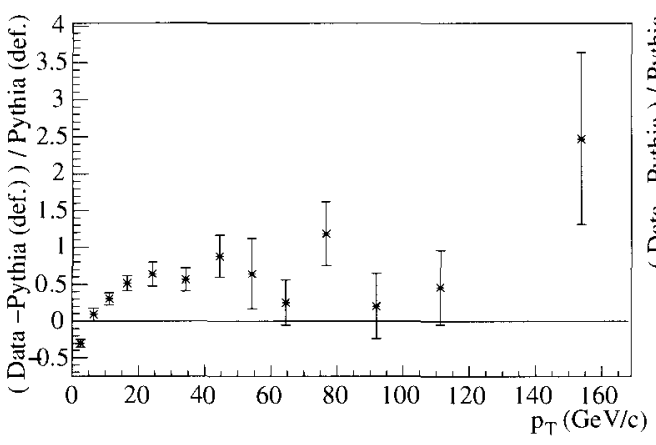

(a)

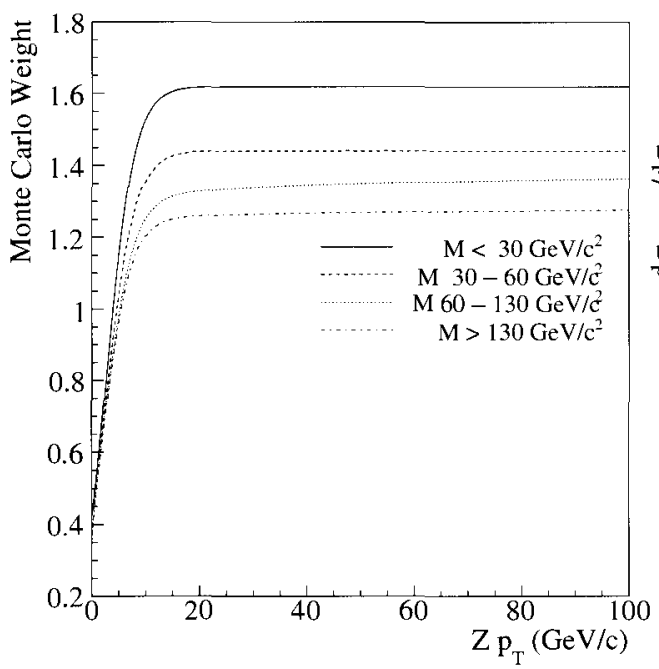

(c)

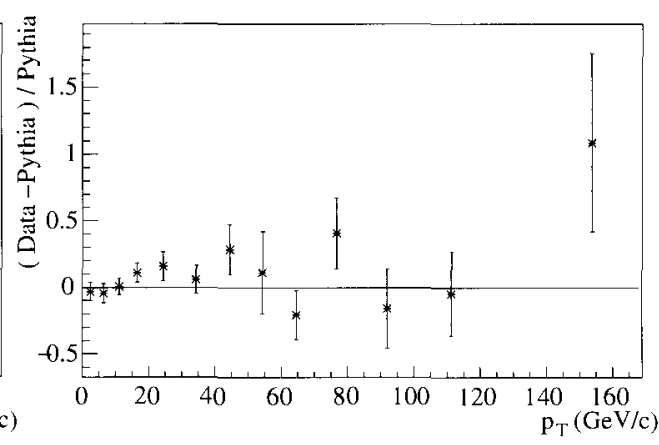

(b)

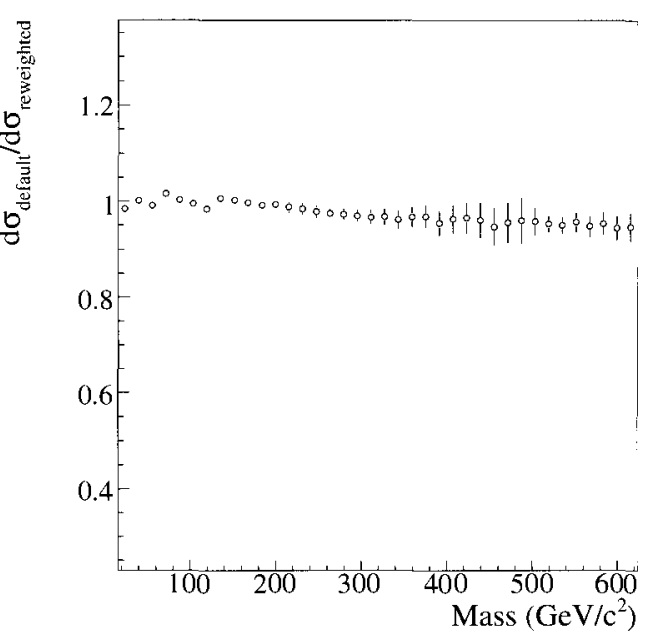

(d)

Figure 4.5: Fractional difference between data and default (a) / tuned (b) PYTHIA versions for the differential cross-section of the $Z$ boson as a function of $p_{T}(Z) ;$ (c) shows the function in equation (4.1) fitted to the fractional difference between tuned and default PyTHIA versions as a function of generated $p_{T}(Z)$ for different mass ranges. The data in these plots is a sample of selected $Z \rightarrow \mu^{+} \mu^{-}$events from $146.9 \mathrm{pb}^{-1}$ of early Run IIa data with $60<M_{\mu \mu}<130 \mathrm{GeV} / \mathrm{c}^{2}$. Reproduced from [80]. (d) shows the ratio of the default and the reweighted Monte Carlo invariant dimuon mass distribution for the entire $\gamma^{\star} / Z$ sample. 


\begin{tabular}{|c|c|c|c|c|}
\hline & \multicolumn{2}{|c|}{$15<M_{\gamma^{*} / Z}<30 \mathrm{GeV} / c^{2}$} & \multicolumn{2}{|c|}{$30<M_{\gamma^{\star} / Z}<60 \mathrm{GeV} / c^{2}$} \\
\hline$p_{0}$ & $1.618 \pm 0.011$ & & $1.441 \pm 0.008$ & \\
\hline$p_{1}$ & $2.692 \pm 0.044$ & $\mathrm{GeV}$ & $2.387 \pm 0.041$ & $\mathrm{GeV}$ \\
\hline$p_{2}$ & $-0.389 \pm 0.006$ & $\mathrm{GeV}^{-1}$ & $-0.365 \pm 0.007$ & $\mathrm{GeV}^{-1}$ \\
\hline$p_{3}$ & 0 & & 0 & \\
\hline & \multicolumn{2}{|c|}{$60<M_{\gamma^{*} / Z}<130 \mathrm{GeV} / c^{2}$} & \multicolumn{2}{|c|}{$M_{\gamma^{\star} / Z}>130 \mathrm{GeV} / c^{2}$} \\
\hline$p_{0}$ & $1.332 \pm 0.007$ & & $1.261 \pm 0.005$ & \\
\hline$p_{1}$ & $1.798 \pm 0.128$ & $\mathrm{GeV}$ & $1.760 \pm 0.123$ & $\mathrm{GeV}$ \\
\hline$p_{2}$ & $-0.357 \pm 0.010$ & $\mathrm{GeV}^{-1}$ & $-0.384 \pm 0.010$ & $\mathrm{GeV}^{-1}$ \\
\hline$p_{3}$ & $0.0194 \pm 0.0057$ & & $0.0100 \pm 0.0054$ & \\
\hline
\end{tabular}

Table 4.2: Parameters of the reweighting function (4.1) for different ranges of $M_{\gamma^{\star} / Z}$.

\section{$Z p_{T}$ reweighting}

As the default version of PYTHIA does not correctly describe the $Z p_{T}$ spectrum in data [80], the PYTHIA output is reweighted according to the description of [80] (as implemented in a standard software package, the ZPtReWeighting tool in caf_mc_util). A 'tuned' parameter set for PYTHIA, which results in a modified distribution of $k_{\perp}$ (the effective transverse momentum of the partons, cf. 2.2.1), was derived to match the Run-I $p_{T}(Z)$ measurement in the $Z \rightarrow e^{+} e^{-}$channel [81]. The following function was then fit to the ratio of the differential cross-section $\frac{\mathrm{d} \sigma}{\mathrm{d} p_{T}}$ of the 'tuned' PYTHIA output to the default,

$$
\frac{\left(d \sigma / d p_{T}\right)_{\text {tuned pythia }}}{\left(d \sigma / d p_{T}\right)_{\text {default pythia }}}=\frac{p_{0}}{1+e^{\left(p_{T}-p_{1}\right) \cdot p_{2}}}+p_{3} \cdot \ln \left(\frac{p_{T}}{20.0 \mathrm{GeV} / c}\right)
$$

and table (4.2) lists the resulting function parameters plus statistical errors for several ranges in $M_{\gamma^{*} / Z}$. Figures 4.5 show the ratio between the measured (unfolded) $Z p_{T}$ spectra in PYTHIA Monte Carlo and data.

While in [80] the fit was derived for $M_{\gamma^{*} / Z}$ up to $250 \mathrm{GeV} / c^{2}$, for this analysis the same weights are applied to $M_{\gamma^{*} / Z}>250 \mathrm{GeV} / c^{2}$ as were derived for $130<M_{\gamma^{*} / Z}<$ $250 \mathrm{GeV} / c^{2}$.

\footnotetext{
${ }^{1}$ The effect on the number of expected background events is in fact not very large, because the main source of background are $Z$-decays with a reconstructed/fitted dimuon mass that is (much) higher than the true value, rather than Drell-Yan events with a true invariant mass $\gg M_{Z}$.
} 


\section{Chapter 5}

\section{Experimental Analysis}

With the simulated backgrounds and signal in place, the first step of the search for heavy resonances in the dimuon spectrum consists of defining a data set and a number of selection cuts which reduce (known and unknown) backgrounds, but preserve signal, in order to increase the sensitivity of a counting experiment to the presence of a signal (see the next chapter).

The main background comes of course from $\gamma^{\star} / Z \rightarrow \mu^{+} \mu^{-}$decays, which have similar characteristics as the signal events except for the invariant mass (and, depending on the model, the opening angle between the muons). Because of this, the cut on this quantity is optimized explicitly - this is presented in the next chapter.

Since the amount of signal events for which $M_{\mu \mu} \sim M_{Z}$ is negligible compared to the expected background, the measured $Z$ peak cross section can be used to determine the effective luminosity around the $Z$ peak, assuming the $Z$ cross section is known.

The first cuts described below are efficient (loose) cuts requiring at least two muon objects in the event with matched high- $p_{T}$ central tracks, one of which is required to be isolated. The efficiencies of these cuts were measured independently on $Z \rightarrow \mu^{+} \mu^{-}$ decays using a tag-and-probe method.

Additional loose cuts further reduce backgrounds and increase the quality of the event sample. The efficiencies of these cuts are determined by looking at the number of $Z$ events in the complement samples (the samples of events which fail one cut but pass all other cuts). Backgrounds which are not included in the simulation are shown to be negligible after these cuts.

\subsection{Data Set}

This analysis is based on the entire "RunIIa" dataset, which was taken between April 2002 and February 2006.

Skim In order to process more quickly the large amount of data included in the entire dataset, events are tagged during reconstruction, which allows one to 'skim' 


\begin{tabular}{|lllll|}
\hline Trigger Lists & Run Range & delivered $\left(\mathrm{pb}^{-1}\right)$ & recorded $\left(\mathrm{pb}^{-1}\right)$ & rec. $+\mathrm{DQ}\left(\mathrm{pb}^{-1}\right)$ \\
\hline v8 - v10.3 & $160582-173101$ & 100.4 & 84.3 & 52.6 \\
v10.3 - v13 & $173352-194597$ & 364.7 & 337.0 & 304.1 \\
v13 & $194567-208500$ & 463.1 & 425.6 & 374.8 \\
v14 & $207217-215670$ & 416.8 & 389.1 & 332.0 \\
\hline & pre-shutdown & 541.2 & 490.2 & 411.6 \\
& post-shutdown & 803.7 & 745.7 & 652.8 \\
\hline & Total & $\mathbf{1 3 4 5}$ & $\mathbf{1 2 3 6}$ & $\mathbf{1 0 6 4}$ \\
\hline
\end{tabular}

Table 5.1: Delivered and recorded integrated luminosity before and after data quality requirements, per trigger list, totals before and after the 2004 shutdown (run number 200000 ), and total.

over the data. The skim ${ }^{1}$ definition used for this analysis requires 2 loose muons with a central track $p_{T}>10 \mathrm{GeV}$ (see below for definition). Because the skim definition is included in the selection, the skimming efficiency is not taken into account separately.

\subsubsection{Data Quality}

Runs that are marked bad for the calorimeter, SMT, CFT or muon systems, because of some problem with the (sub)detector,are removed from the data using standard tools. Events and luminosity blocks (see 3.2.2) which had been marked as displaying known patterns of calorimeter noise were removed as well. Together these data quality requirements remove about $13 \%$ of the initial data sample (see table 5.1).

\subsubsection{Luminosity}

Table 5.1 lists the delivered luminosity, the recorded luminosity and recorded with data quality requirements, per trigger list, in total and before and after the 2004 shutdown (a trigger list is a combination of triggers used in contiguous runs). The integrated luminosity, as measured by the luminosity system, was determined for the

\footnotetext{
${ }^{1}$ The Common Sample Group skims that were used correspond to the following SAM dataset definitions for the ROOT-tuples:

- CSG_CAF_2MUhighpt_PASS3_p17.09.06

- CSG_CAF_2MUhighpt_PASS3_p17.09.06b

- CSG_CAF_2MUhighpt_PASS3_p17.09.03

The suffixes denote the version of the doreco program that were used to reconstruct the events in that part of the skim. The ROOT-tuples were created with TMBAnalyze p18.05.00. In addition, duplicate events that occurred due to mistakes in the reconstruction process were removed from the skim. Versions caf_dq v02-01-01 and dq_defs v2006-11-30 were used to apply data quality criteria.
} 
2MUhighpt skims for an unprescaled trigger which was used during the entire data taking period; the trigger that was used is JT_ $125 \mathrm{TT}$.

These numbers are not used in the calculation of the cross-section limits, but only as a consistency check to the calculation of the integrated effective luminosity.

\subsubsection{Triggers}

To ensure the highest possible trigger efficiency, the events are required to have fired any single muon or dimuon trigger in a trigger list (a trigger list is a collection of trigger requirements). These triggers typically require a number of PDT and/or scintillator hits, muon tracks, and possibly a (matched) track. A few triggers in v14 require an isolated track at level 3 . Table B.1 on page 124 lists all used triggers per trigger list period.

Since the expected contribution from signal (i.e., $Z^{\prime}$ decays) is negligible for events with an invariant dimuon mass around the $Z$ mass, assuming a known $Z$ peak cross section the Monte Carlo can be normalized to the measured $Z$ cross section in order to find the effective luminosity $\mathcal{L}_{\text {eff }}=$ acceptance $\cdot$ efficiency $\cdot \mathcal{L}$. This is explained in section 5.4.1.

\subsection{Muon Reconstruction}

Offline muon objects are identified by using information from the muon detector system, in combination with the central tracker and/or the calorimeter.

For each layer of the muon system, an attempt is made to fit hits into track stubs called segments. Where segments can be matched together, they are fitted into a local muon track.

For muon segments with A-layer hits in the central region of the muon detector $(|\eta|<1)$, PDT pad information is used to improve the resolution in the $\phi$ direction (see below).

Various muon object quality definitions exist, and are detailed in [82]. They differ with respect to the number of required drift tube and scintillator hits and the number of layers in which hits occur. For maximal efficiency, this analysis uses only 'loose' muons that are matched to a central track. A central track-matched muon object is called 'loose' if it satisfies at least one of the following hit requirements:

- One A-layer scintillator hit and 2 A-layer wire hits; or

- One BC-layer scintillator hit and 2 BC-layer wire hits

In addition to the loose hit requirements, the muons are required to have their scintillator hit(s) occur within $10 \mathrm{~ns}$ of the beam crossing time, to reject cosmic events and ensure nominal tracking efficiencies. This does not remove all cosmic muons in the sample, but the muon ID efficiencies are measured relative to this cut. See the discussion in section 5.4.5 for further cuts to reject cosmic muons. 


\begin{tabular}{|ccl|}
\hline Octant & $\sigma$ (no pads) $(\mathrm{cm})$ & $\sigma$ (pads) $(\mathrm{cm})$ \\
\hline 1 & $10.14 \pm 0.098$ & $5.32 \pm 0.046$ \\
2 & $9.70 \pm 0.092$ & $5.23 \pm 0.045$ \\
3 & $10.06 \pm 0.097$ & $4.94 \pm 0.039$ \\
4 & $9.88 \pm 0.098$ & $4.66 \pm 0.039$ \\
5 & $10.18 \pm 0.097$ & $4.63 \pm 0.038$ \\
6 & $10.65 \pm 0.12$ & $7.93 \pm 0.20$ \\
7 & $10.31 \pm 0.12$ & $8.46 \pm 0.11$ \\
8 & $10.47 \pm 0.10$ & $5.24 \pm 0.046$ \\
\hline
\end{tabular}

Table 5.2: $\sigma$ of a Gaussian fitted over $\mu \pm 3 \sigma$ of the difference between the reconstructed muon track position in the direction along the wire at the A-layer and the extrapolated central track position, per octant, with and without using the PDT pad information. Only muons with at least 2 good pad readouts are considered. Errors are statistical only.

\section{PDT pad reconstruction}

The muon segment position is precisely measured in the directions orthogonal to the drift chamber wires (thus, parallel to the beam direction), but not in the direction along the wires. A rough estimate is obtained by using the position of the scintillator (with a length of approximately $60 \mathrm{~cm}$ ) and from the delay between signals measured in two adjacent, connected PDTs ('axial time') (see section 3.2.5).

Inside a PDT, charge is collected on an inner cathode pad and an cathode outer pad (see section 3.2.5). Because the pads are tapered, the ratio of the charge collected by the two pads provides information on the position along the wire. Since the tapered geometry of the pads is repeated 11 times along the wire, there is a remaining 11-fold ambiguity. This ambiguity can be resolved by comparing the position measurements of more than one deck and the scintillator / axial time measurements.

By using the pad information, the estimate of the local muon track position is improved (but note that in this analysis, the muon track position is used only for matching the muon to a central track). In order to assess the position resolution improvement obtained by using the pad information, a sample of $Z \rightarrow \mu^{+} \mu^{-}$events was taken from Run IIa data. The reconstructed muon object position was then compared with the position of the central track, extrapolated to the A-layer.

The events were selected requiring two muon objects, with at least 1 scintillator and 2 wire hits in the A-layer and at least 1 scintillator and 3 wire hits in the $\mathrm{B}+\mathrm{C}$ layers, matched to central tracks with a $p_{T}>15 \mathrm{GeV} / c$, and an invariant dimuon mass between 70 and $110 \mathrm{GeV} / c^{2}$. One of the two muons was required to be well within the nominal geometric acceptance for the PDTs. To make sure the muon was matched to the right track, no other tracks were allowed to be within a cone of $\Delta R(\eta, \phi)<0.5$ (see below for definitions). 
Table 5.2 lists the position resolution in each octant of the central muon system, before using PDT pads and after, for muons that had at least 2 good pads read out; this happens in about $65 \%$ of the muons, except for octants 6 and 7 where it happens only for about $50 \%$. Octant 6 and 7 are the bottom octants in which some instrumentation is missing due to the presence of the support beams in the 'bottom hole'.

\subsubsection{Central track matching}

The loose muons are required to be matched to a central track. The central track can have hits in both the SMT and the CFT, or be CFT-only. The central track resolution is much better than the local muon track resolution.

Even for tight muons (with wire and scintillator hits in every layer and a converged local muon track fit), the local muon track $p_{T}$ resolution for muons with a $p_{T} \sim$ $10 \mathrm{GeV}$ is around $25 \%$ (WAMUS, wide-angle or central part of the muon system) or $20 \%$ (FAMUS, forward part of the muon system), for muons with a $p_{T} \sim 40 \mathrm{GeV}$ it is around $40 \%$. The loose muons required for this analysis may have a local muon track which is not fit through all layers (and hence have a significantly worse local muon $p_{T}$ resolution) or which did not converge for some reason. Since the muon $p_{T}$ is taken from the central track, the more efficient loose muon definition can be used without worsening the resolution (and hence the sensitivity). In addition the analysis is less sensitive to the degree of accuracy to which the muon system geometry is modeled in the detector simulation.

Muons are matched to central tracks by considering all possible matches between muons (both local muon tracks and single segments) and central tracks propagated to the muon system, and also all matches between central tracks and muons propagated to the central track's point of closest approach to the center of the detector. The unique match with the lowest $\chi^{2}$ (of all 5 track parameters) is used. No cut on the matching $\chi^{2}$ is performed. The track matching efficiency is typically on the order of $99 \%,[82]$; it is however not considered separately from the muon ID efficiency.

The track that is matched to the muon object, also loose, is required to have a distance of closest approach (dca) to the primary vertex of at most $0.02 \mathrm{~cm}$ if the track has at least one hit in the SMT, or $0.2 \mathrm{~cm}$ if it is a CFT-only track. These are loose cuts; the typical resolution for muons coming from $Z$ decays are $20 \mu \mathrm{m}$ and $500 \mu \mathrm{m}$ respectively

\subsubsection{Efficiency measurements}

The muon ID group has measured the loose muon identification times track matching times cosmic veto efficiency and the loose track efficiency, as well as the isolation efficiency on $Z \rightarrow \mu^{+} \mu^{-}$data found in a selected sample from the same dataset used for the analysis, using a tag-and-probe method. The details can be found in [82].

To measure an efficiency with the tag-and-probe method, one requires the control or "tag" muon to satisfy tight selection criteria, designed to filter out all but the $Z \rightarrow \mu^{+} \mu^{-}$events, on both the muon object and the track. 


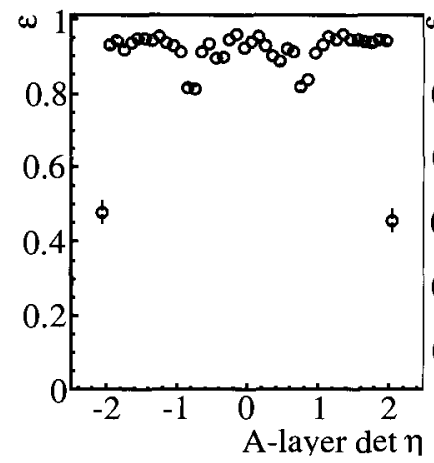

(a)

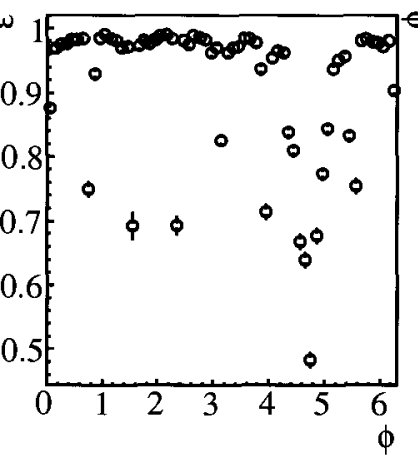

(b)

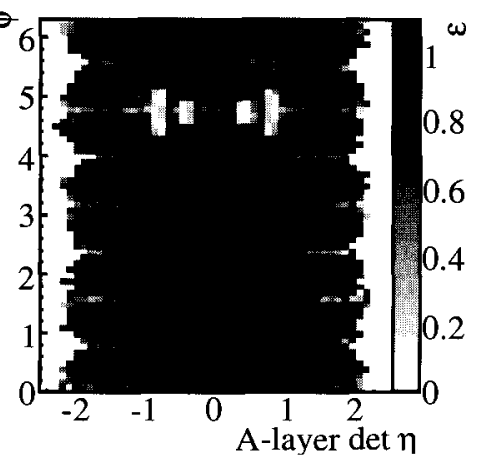

(c)

Figure 5.1: Loose muon ID efficiency in data as a function of the muon (a) pseudorapidity and (b) azimuthal angle at the A-layer of the muon system, and both (c).

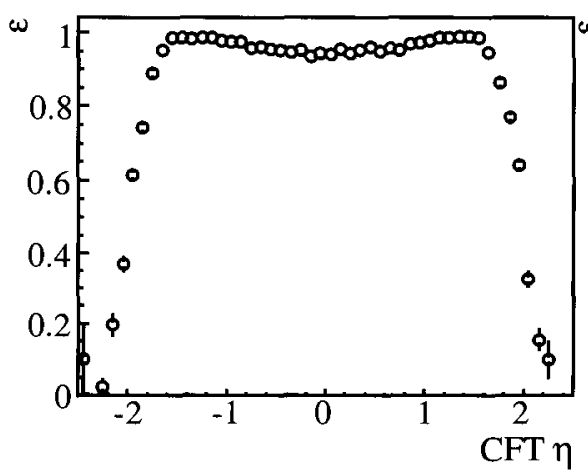

(a)

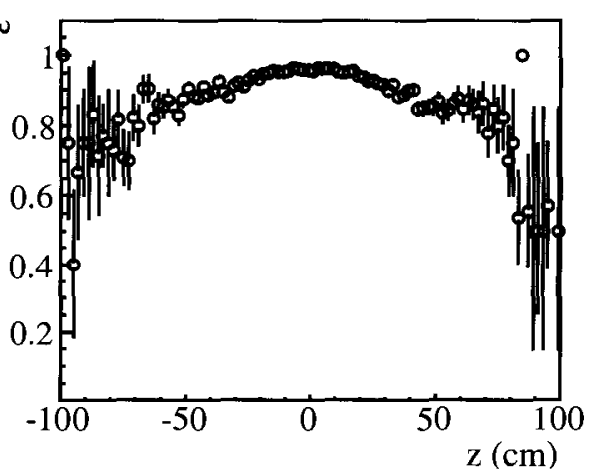

(b)

Figure 5.2: Loose track efficiency in data as a function of (a) the (detector) pseudorapidity of the track at the outermost layer of the CFT (at a radius of $51.43 \mathrm{~cm}$ ) and (b) the $z$ position at the detector center. 
The "probe" object is then required to pass all criteria except the one of which the efficiency is being measured; also, the event topology should match that of a $Z \rightarrow$ $\mu^{+} \mu^{-}$event. One then looks at the fraction of events in which the "probe" object passes that selection as well.

When measuring the muon ID $\times$ track matching $\times$ anti-cosmic timing efficiency, as the "probe" one requires a second track satisfying the same criteria as the "tag", and the tracks are required to be back-to-back in the transverse plane only and be isolated in the calorimeter and in the tracker (see below). The efficiency is measured as a function of muon $\eta$ and $\phi$. The results for data are shown in figures 5.1

When measuring the track reconstruction times $\mid$ dcal (distance of closest approach) cut efficiency, the "probe" is a muon object which is well separated from the "tag" muon, has a local muon transverse momentum $p_{T \text { local }}>15 \mathrm{GeV}$ and a scintillator hit time difference $|\Delta t|<6$ ns relative to the "tag" muon. The tracking efficiency is measured as a function of the $z$-position of the track at the (3-dimensional) distance of closest approach to the primary vertex. The results for data are shown in figures 5.2 .

The efficiencies are measured on data and on Monte Carlo. Because the Monte Carlo does not reproduce the measured efficiencies accurately, it is reweighted with parameterized correction factors; these are presented in section 5.3 .

\subsubsection{CFT-only track correction}

CFT-only tracks, i.e., central tracks with no hits in the SMT, are corrected using the run-average beam spot position and uncertainty. (The beam spot is the region of interaction between the colliding beams.)

The reason that the primary vertex is not used to correct tracks (with or without SMT hits) is that a typical high- $p_{T}$ dimuon event has only a few tracks coming from the true primary vertex (namely, the central tracks of the two muons). Therefore, the primary vertex position and error can be biased towards the muon track vertex.

The run-average beam spot position is reconstructed (using the AATrack package [83]) using from each event the vertex with the maximal track multiplicity, with at least 3 tracks. A given run must have at least 5 events with such a vertex for a beam spot measurement to be made.

For each CFT-only track, the distance of closest approach to the beam spot, dcabeam, is computed as

$$
\text { dcabeam }=r_{\text {dca, signed }}-\left(x_{\text {beam }}+\Delta x_{\text {beam }} \cdot z\right) \cdot \sin \phi+\left(y_{\text {beam }}+\Delta y_{\text {beam }} \cdot z\right) \cdot \cos \phi
$$

where $r_{\mathrm{dca}, \text { signed }}$ and $z$ are the track's signed radial impact parameter $\left(r_{\mathrm{dca}}\right.$, signed $=$ $r_{\mathrm{dca}} \cdot \operatorname{sign}\left(\phi_{\text {position }}-\phi_{\text {direction }}\right)$ and $z$-coordinate at the distance of closest approach to the center of the detector, $\phi$ is the track azimuthal angle, $x, y_{\text {beam }}$ is the position of the beam spot at $z=0 \mathrm{~cm}$ and $\Delta x, y_{\text {beam }}$ is the slope of the beam. The track curvature is then corrected as 


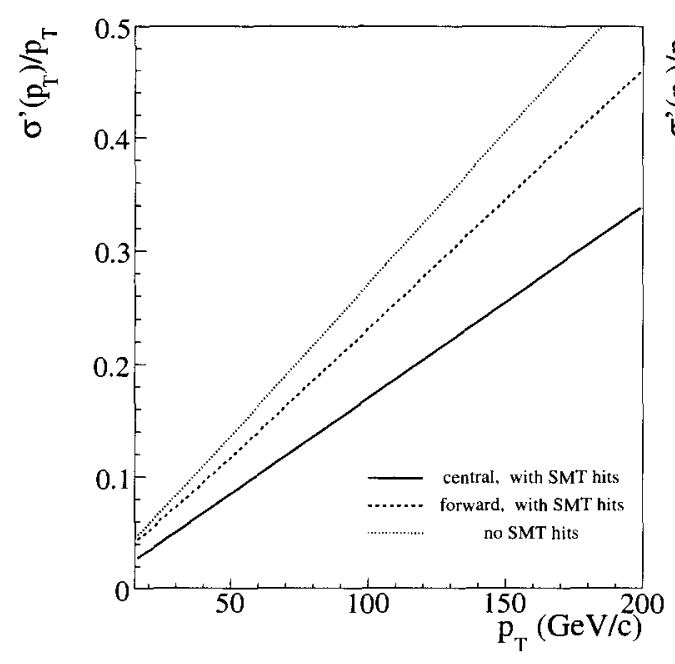

(a)

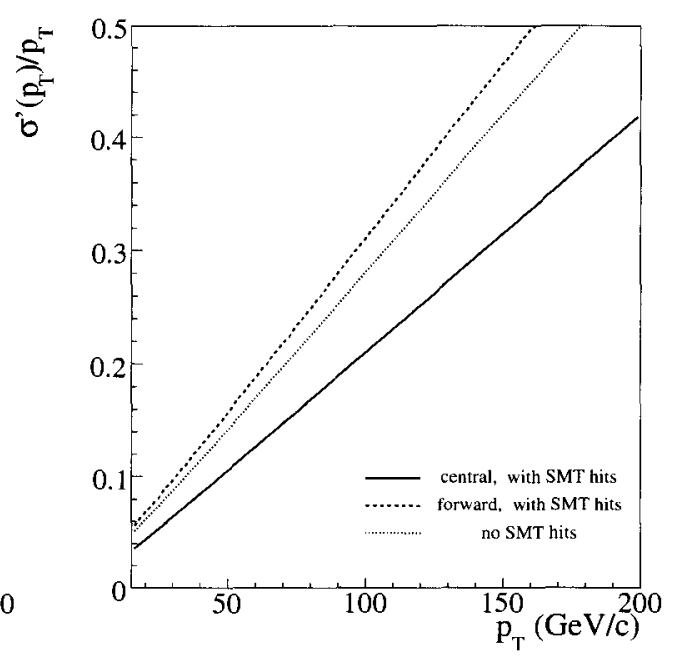

(b)

Figure 5.3: Relative additional resolution $\sigma^{\prime} / p_{T}$ (see equation (5.1)), (a) pre-shutdown and (b) post-shutdown. Central muons with $\left|\eta_{C F T}\right|=0$, forward muons with $\left|\eta_{C F T}\right|=1.6$.

$$
q / p_{T} \rightarrow q / p_{T}-\text { dcabeam }_{\text {bea }} \cdot \frac{\sigma_{r \frac{q}{p_{T}}}}{\sigma_{r r}}
$$

where $q$ is the charge and $p_{T}$ the transverse momentum, $\sigma_{r \frac{q}{p_{T}}}$ is the correlation between the curvature and the radial impact parameter and $\sigma_{r r}$ is the uncertainty on the latter.

For runs for which, for whatever reason, no beam position measurement was found in the beam spot database, the measurement from the nearest run in the same store is used instead, since the beam position does not in general experience significant shifts during a store. This happens in only 47 events out of 6 runs in the final sample.

In the Monte Carlo, the center of the detector is used as the beam spot position.

\subsection{Muon track $p_{T}$ resolution correction in Monte Carlo}

The detector simulation uses an idealized geometry of the tracking system and some of the dead material is not implemented in the simulation. Because of this, and possibly other unknown effects, the resolution of the transverse momentum of the muons is overestimated. Therefore, the muon transverse momentum is 'smeared' to fix the detector simulation. This correction becomes large for highly energetic muons (see 


\begin{tabular}{|lccc|}
\hline & & $\mathrm{A}\left(10^{-3} \mathrm{GeV}^{-1}\right)$ & $\mathrm{B}\left(10^{-2}\right)$ \\
\hline central, & pre-shutdown & $1.7 \pm 0.1$ (stat) \pm 0.1 (syst) & $0.9 \pm 0.3$ (stat) \pm 0.4 (syst) \\
$\geq 1$ SMT hits & post-shutdown & $2.1 \pm 0.2$ (stat) \pm 0.4 (syst) & $1.2 \pm 0.2$ (stat) \pm 0.3 (syst) \\
\hline forward, & pre-shutdown & $2.3 \pm 0.4$ (stat) \pm 0.5 (syst) & $1.5 \pm 0.6$ (stat) \pm 0.7 (syst) \\
$\geq 1$ SMT hits & post-shutdown & $3.1 \pm 0.4$ (stat) \pm 0.6 (syst) & $1.7 \pm 0.5$ (stat) \pm 0.5 (syst) \\
\hline no SMT hits & pre-shutdown & $2.7 \pm 0.3$ (stat) \pm 0.3 (syst) & $2.1 \pm 0.6$ (stat) \pm 1.1 (syst) \\
& post-shutdown & $2.8 \pm 0.6$ (stat) \pm 1.0 (syst) & $2.5 \pm 0.9$ (stat) \pm 1.1 (syst) \\
\hline
\end{tabular}

Table 5.3: Muon smearing parameters, defined in equation 5.1. 'Shutdown' refers to the 2004 shutdown.

figure 5.3) and the uncertainty on the track resolution is one of the main systematic uncertainties on the background prediction.

The smearing procedure consists of replacing, for a given muon in an event, the reconstructed (or corrected, cf. section 5.2.3) $q / p_{T}$ of the central track matched to the muon with the 'smeared' $q / p_{T}$ which is sampled from the following distribution,

$$
\frac{q}{p_{T}}+A G_{1}+\frac{B \sqrt{\cosh \eta}}{p_{T}} G_{2}
$$

where $q$ is the reconstructed charge, $p_{T}$ is the original reconstructed transverse momentum, $\eta$ is the reconstructed track (physics) pseudo-rapidity and $G_{1,2}$ are two independent normal distributions.

In order to derive the values for the parameters $A$ and $B$, the predicted reconstructed widths of both the $Z$ and $\mathrm{J} / \psi$ resonance were compared with the resonance width observed in dimuon data. The parameters are derived for muons with hits in the SMT and with either $\left|\eta_{C F T}\right|<1.6$ ('central') or $\left|\eta_{C F T}\right|>1.6$ ('forward'), where $\eta_{C F T}$ is the (detector) pseudo-rapidity of the track at the outermost layer of the CFT (at a radius of $51.43 \mathrm{~cm}$ ); and for muons without hits in the SMT. In addition, the parameter values were derived separately for data taken before and after the fall 2004 shutdown (see figure 3.2), since it was observed that the track resolution changed significantly over the shutdown [82] (for unknown reasons). For each period, the parameters are determined on three separate samples, with the two selected muons either both with SMT hits and both central, both with SMT hits and one muon forward, or with one muon with SMT hits and one without. Table 5.3 lists the parameters derived for each muon type and period.

Figure 5.3 shows the relative additional resolution

$$
\sigma^{\prime}\left(p_{T}\right) / p_{T}=\sqrt{A^{2} p_{T}^{2}+B^{2} \cosh \eta}
$$



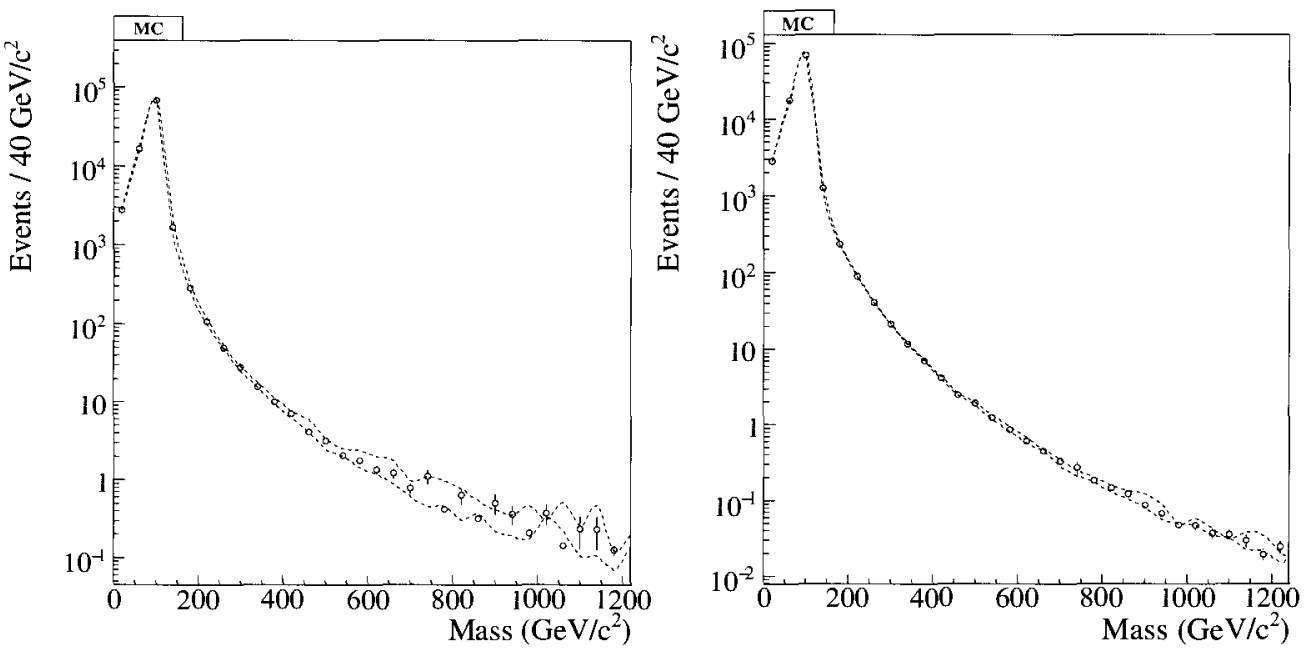

Figure 5.4: (a) Reconstructed Monte Carlo dimuon invariant mass distribution, normalized to the $Z$ peak in data (markers), and the uncertainties on the normalized distribution due to the statistical plus systematic uncertainties on the muon resolution, cf. table 5.3 (dashed lines). The error bars represent the statistical uncertainty on the number of (weighted) Monte Carlo events in a bin.

(b) The same distribution after the kinematic fit described in section 5.5.

\subsection{1 $p_{T}$ resolution uncertainties}

The statistical and systematic uncertainties on the smearing parameters are taken into account as a systematic uncertainty on the expected background. Systematic uncertainties that were taken into account are effects due to the variation of the fit ranges around the $Z$ and $\mathrm{J} / \Psi$ peaks, variation of the shift of the reconstructed invariant mass, variation of the muon and track quality requirements and $p_{T}$ cut.

Figure 5.4 shows the uncertainty propagated to the invariant mass distribution for the combined Monte Carlo background sample. This uncertainty is one of the largest systematic uncertainties, but it is reduced after a kinematic fit, which will be described in section 5.5 .

\subsection{Event selection}

The signal final state is expected to contain opposite-sign muons with high invariant mass coming from the same vertex. The dimuon system is expected to be boosted in the longitudinal direction because of the boost of the parton-parton system, but the muons are expected to be roughly back-to-back in the transverse plane in the majority of the events. The muons are expected to be isolated, i.e., they leave little energy in the calorimeter and have few or low-energetic tracks around them, but a highly energetic muon can radiate and thus be non-isolated in the calorimeter. 
In the following, the Standard Model Monte Carlo (described in the previous chapter), with the aforementioned $K^{(\mathrm{QCD})}$-factor and $Z p_{T}$ corrections applied, is weighted with parameterized muon, track and isolation efficiency corrections as described above. The corrected background Monte Carlo is normalized to the number of $Z$ events in data, counted with a binned likelihood fit, described here.

A series of one dimensional selection cuts is applied. As a verification of the simulation, the efficiency of each cut on $Z \rightarrow \mu^{+} \mu^{-}$events is compared between the Standard Model Monte Carlo and data.

\subsubsection{Z peak fit}

The number of $Z$ events is estimated by a binned likelihood fit on the dimuon invariant mass distributions around the $Z$ mass. The fitted function is a Breit-Wigner resonance shape plus exponential falling background convoluted with a single (shifted) Gaussian with mean $\Delta$ and width $\sigma$, i.e.,

$$
\begin{aligned}
& f\left(m ; A_{\text {signal }}, A_{\mathrm{bkg}}, \Delta, \sigma, s\right)= \\
& \int_{0}^{\infty} d x \frac{1}{\sqrt{2 \pi} \sigma} e^{-\frac{1}{2}\left(\frac{x-\Delta}{\sigma}\right)^{2}} \times( A_{\mathrm{bkg}} \cdot e^{-s \cdot\left(M_{Z}-m+x\right)} \\
&\left.+A_{\text {signal }} \cdot \frac{1}{\Gamma_{Z}^{2}+\left(M_{Z}-m+x\right)^{2}}\right)
\end{aligned}
$$

where $M_{Z}$ and $\Gamma_{Z}$ are the $Z$ boson mass and width respectively (and are kept fixed). The number of $Z$ events is counted as the integral over the "peak" part of the function

$$
N_{Z}=\left.\int_{M_{Z}+\Delta-5 \cdot \sigma}^{M_{Z}+\Delta+5 \cdot \sigma} d m f(m ; \ldots)\right|_{A_{\mathrm{bkg}}=0}
$$

The fit error on this quantity is then computed as the fit error on the integral over the complete (peak and background) function, keeping the size of the interval fixed at $10 \sigma$.

In sections 5.4.2ff., we will make a number of selection cuts. For the events failing the cut, the dimuon invariant mass will be shown with a fit to the $Z$ peak as described above. The results of the fits are shown with a dashed error band corresponding to the (approximate) $1 \sigma$ confidence intervals of the fit. Also indicated are $N_{Z}$ and the efficiency $\epsilon$ relative to $N_{Z}$ measured on the events passing all cuts (see below). The Monte Carlo histograms depict the mass distributions for all background samples, corrected to reproduce the muon ID, track reconstruction and muon isolation efficiencies as described above.

The selection efficiency on $Z \rightarrow \mu^{+} \mu^{-}$events for each single cut (other than the muon ID, track ID and isolation efficiencies, of which the efficiencies are estimated using the tag-and-probe method) is estimated by counting the number of events coming from a $Z$ decay that fail only that cut. The selection efficiencies are measured on the data as 


$$
\epsilon=\frac{N_{Z, \text { pass }}}{N_{Z, \text { fail }}+N_{Z, \text { pass }}}
$$

where $N_{Z}$, fail is the number of $Z$ events failing that specific cut but passing all other cuts and $N_{Z}$, pass the number of $Z$ events passing all cuts. The uncertainty on $\epsilon$ is then calculated simply by error propagation of the independent fit errors $\Delta N_{Z}$, fail, $\Delta N_{Z, \text { pass }}$

$$
(\Delta \epsilon)^{2}=\frac{\left(\Delta N_{Z, \text { fail }}\right)^{2} N_{Z, \text { pass }}^{2}+\left(\Delta N_{Z, \text { pass }}\right)^{2} N_{Z, \text { fail }}^{2}}{\left(N_{Z, \text { pass }}^{2}+N_{Z, \text { fail }}^{2}\right)^{2}}
$$

this neglects all correlations, and is therefore an upper limit.

The efficiencies in data and their uncertainties are found to be compatible with the same quantities calculated on the Monte Carlo,.

\section{Normalization}

The normalization $n$ is calculated, for the measured and predicted invariant mass distribution after the kinematic fit, as

$$
n=\frac{N_{Z, \text { data }}}{\sigma_{\mathrm{eff}}(Z)}
$$

where

$$
\sigma_{\mathrm{eff}}(Z)=\epsilon_{\mathrm{MC}}(Z) \cdot \sigma_{Z \rightarrow \mu^{+} \mu^{-}}
$$

Here the Monte Carlo $Z \rightarrow \mu^{+} \mu^{-}$event selection efficiency $\epsilon_{M C}(Z)$ is determined with the binned likelihood fit to the $Z$ peak in the full Standard Model Monte Carlo (i.e., including all samples). $\sigma_{Z \rightarrow \mu^{+} \mu^{-}}$is the inclusive theoretical NNLO (QCD) $Z$ production cross section times branching ratio [78],

$$
\sigma_{Z \rightarrow \mu^{+} \mu^{-}}(\mathrm{CTEQ} 6.1 \mathrm{M})=241.59 \mathrm{pb}{ }_{-7.7}^{+8.6}(\mathrm{PDF})_{-0.7}^{+0.6}(\text { scale })
$$

where the positive and negative errors due to the PDF uncertainty and the factorization / renormalization scale errors are indicated (see section 4.2).

The uncertainty on the normalization is calculated as

$$
\left(\Delta^{ \pm} n\right)^{2}=\left(\frac{\Delta N_{Z, \text { data }}}{\sigma_{\text {eff }}(Z)}\right)^{2}+\left(\Delta^{ \pm} \sigma_{\text {eff }} \frac{N_{Z, \text { data }}}{\sigma_{\text {eff }}^{2}}\right)^{2}+\left(\delta_{\text {kin.fit. }}\right)^{2}
$$

where $\Delta N_{Z \text {, data }}$ is the fit error, $\delta_{\text {kin.fit. }}=\mid n$ (reconstructed) $-n$ (kin. fit.) $\mid$ is the difference of $n$ calculated for the fits to the reconstructed mass distributions with the normalization calculated for the fits to the mass distributions after the kinematic fit, (see section 5.5) and 


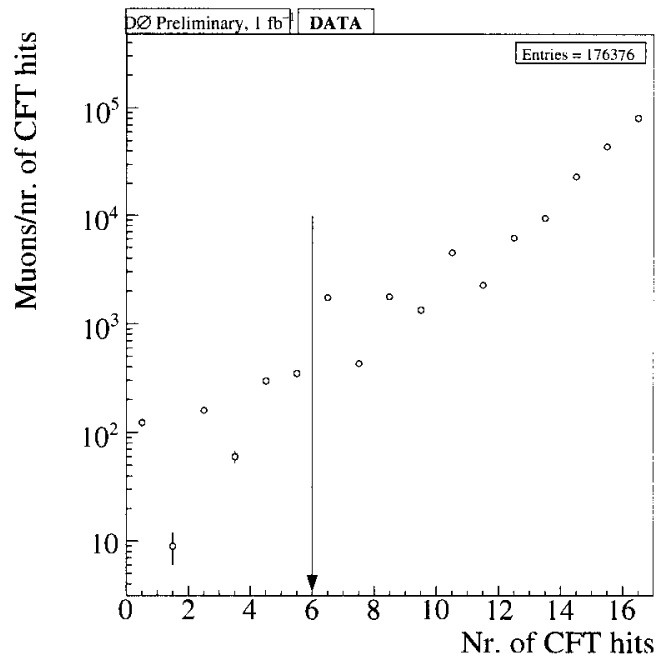

Figure 5.5: Number of CFT hits on muon central tracks, after all other cuts have been applied, for events with $M_{\mu \mu}>40 \mathrm{GeV} / c^{2}$.

$$
\Delta^{ \pm} \sigma_{\text {eff }}=\sqrt{\Delta \epsilon_{M C}(Z)^{2}+\Delta^{ \pm} \sigma_{Z \rightarrow \mu^{+} \mu^{-}}^{2}}
$$

where $\Delta \epsilon_{M C}(Z)$ is the fit error.

The final result is then

$$
N_{Z, \text { data }}=78561 \pm 1144
$$

(see figure $(5.22(\mathrm{a}))$ ), resulting in

$$
n=1.15 \mathrm{fb}^{-1} \pm 0.025 \text { (fit) } \pm 0.014 \text { (reco-kin.fit) }{ }_{-0.037}^{+0.041} \text { (theory), } \quad\left({ }_{-0.047}^{+0.050} \text { total }\right)
$$

This number is compatible with the integrated luminosity as measured by the luminosity system (see table 5.1 ), $\mathcal{L}=1.064 \pm 0.064 \mathrm{fb}^{-1}$ for a high trigger efficiency, as expected (the most probable value of the trigger efficiency is 1 ).

\subsubsection{Track quality}

- number of CFT hits $\geq 6$

Figure 5.5 shows the number of CFT hits of events passing all other cuts. As can be seen when the reconstructed width of the $Z$ peak for events exclusively failing this cut (figure 5.6) is compared with that of the selected events (figure 5.19) $(14.0 \mathrm{GeV}$ vs. $7.2 \mathrm{GeV}$ ) the invariant mass resolution for these events is considerably worse than 


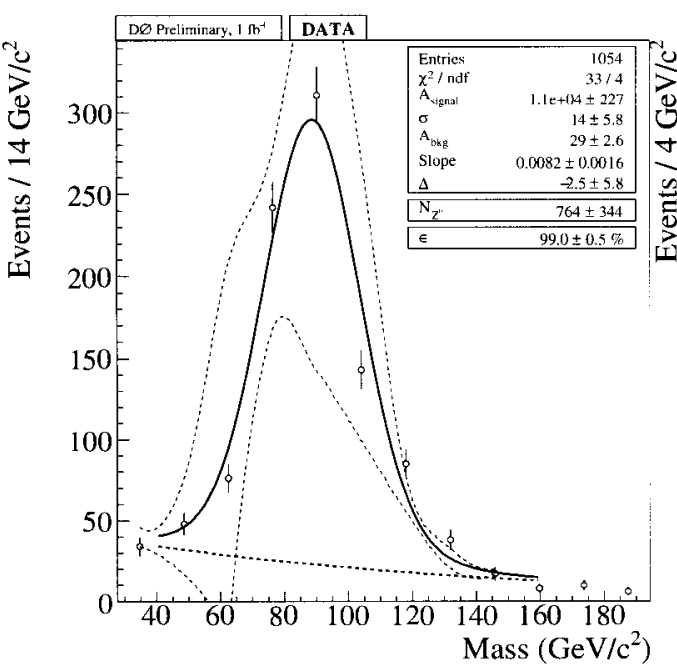

(a)

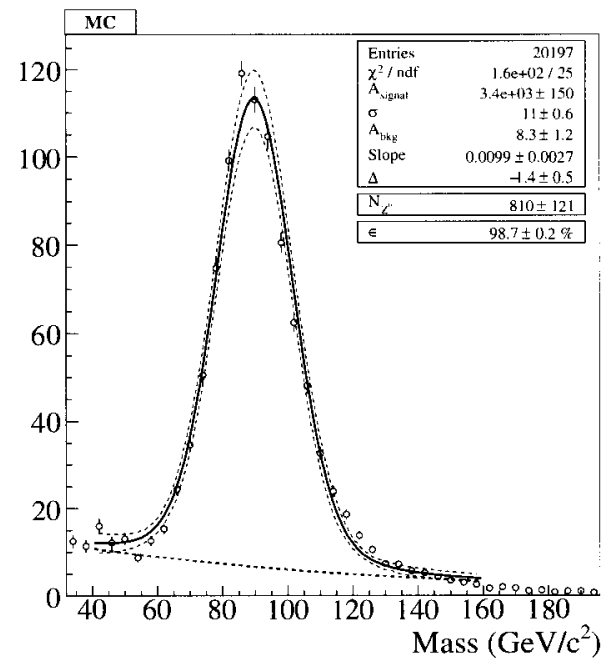

(b)

Figure 5.6: Dimuon mass for events exclusively failing the cut on the number of CFT hits, in data (a) and Monte Carlo (b). The fit in (a) is not well constrained, which is reflected in the large $1 \sigma$ confidence interval.

for events with at least 6 hits in the CFT. In addition, the mass resolution is not well modeled in the Monte Carlo (even after the correction described in 5.3). The efficiency of this cut (on data) for events in the $Z$ peak is $99 \pm 0.5 \%$.

\subsubsection{Isolation}

Of the selected muons, one muon in a muon pair is required to be isolated in the tracker and in the calorimeter. This cut suppresses muons coming from heavy quark jets, since these will have tracks and energy deposits coming from other particles in the jet close to the muon track.

Although neither muon in a signal event is expected to originate from a jet, only one muon out of a muon pair is required to satisfy these isolation criteria. This is because at higher energies, it becomes increasingly likely that at least one muon will lose a significant amount of energy in the calorimeter by Bremsstrahlung, which means that a tight isolation cut on the second muon would lead to a loss of sensitivity.

Specifically, it is required that, first

$$
\sum_{\Delta R<0.5}\left|p_{T}^{(\text {track })}\right|<2.5 \mathrm{GeV}
$$

Here, the sum is over the (other) tracks within a cone of $\Delta R$ (track, muon track) $<0.5$ around the muon track, where $\Delta R(\phi, \eta)=\sqrt{\Delta \phi^{2}+\Delta \eta^{2}}$ and $\Delta(\phi, \eta)$ is the difference with the muon track angle; this sum is called the "track halo". Secondly, 


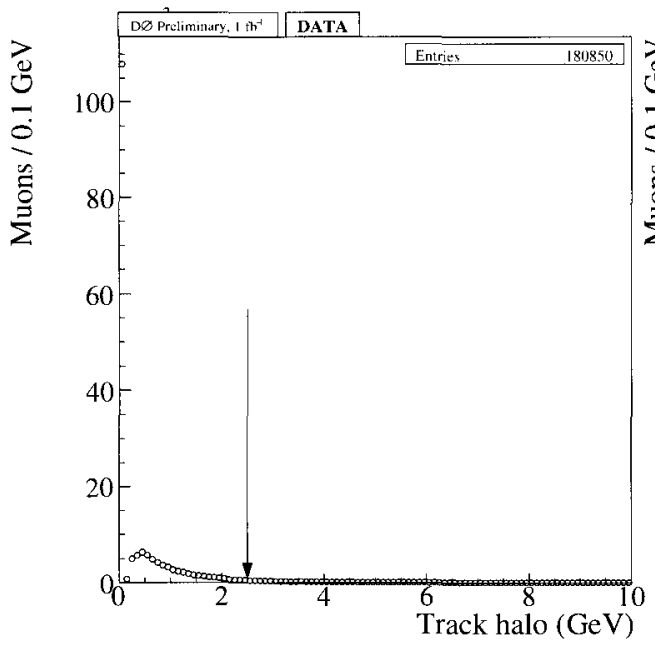

(a)

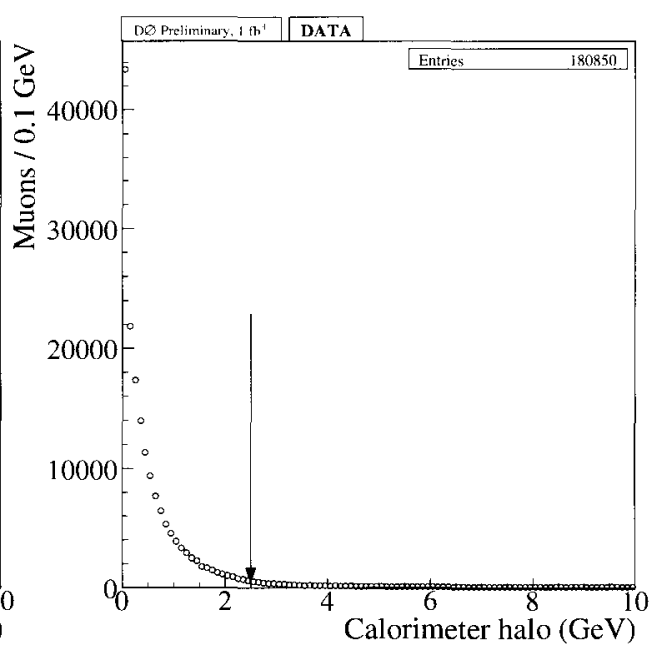

(b)

Figure 5.7: (a) Track halo (cf. equation (5.2)) and (b) calorimeter halo (b) (cf. equation (5.3)), for events passing the cuts on other quantities.

$$
\sum_{0.1<\Delta R<0.4} E_{T}^{(\text {cell })}<2.5 \mathrm{GeV}
$$

where here the sum is over the calorimeter cells with a hollow cone of $0.1<\Delta R$ (track, cell $)<0.4$ around the muon track extrapolated to the calorimeter; this is called "calorimeter halo". Figures 5.7(a) and 5.7(b) show the track and calorimeter halo for events passing all other cuts.

Efficiency determination The efficiency of the isolation cut was determined by the muon ID group [82] with the tag-and-probe method, similar to the way the muon ID and tracking efficiencies were determined (cf. section 5.2.2). The "tag" muon was

required to have $\sum_{\Delta R<0.5}\left|p_{T}^{(\text {track })}\right|<2.5 \mathrm{GeV}$ and $\sum_{0.1<\Delta R<0.4}\left|E_{T}^{(\text {cell })}\right|<3.5 \mathrm{GeV}$. To ensure that the background from heavy flavor quark decays was negligible, the dimuon invariant mass $M_{\mu \mu}$ was required to lie between 80 and $110 \mathrm{GeV}$.

The dependence of the efficiency on the number of jets with an $E_{T}>15 \mathrm{GeV}$ is taken into account when applying the efficiency correction (see figure 5.8(a)). There is a small muon $p_{T}$ dependence for $p_{T}>20 \mathrm{GeV} / c$ (see figure $5.8(\mathrm{~b})$ ) but it is wellmodeled in Monte Carlo [82].

\section{Remaining non-isolated background}

An estimate of the remaining non-isolated background (mainly $Z \rightarrow b \bar{b}$ ) can be made by fitting the selected $Z$ peak plus non-isolated background shapes to events 


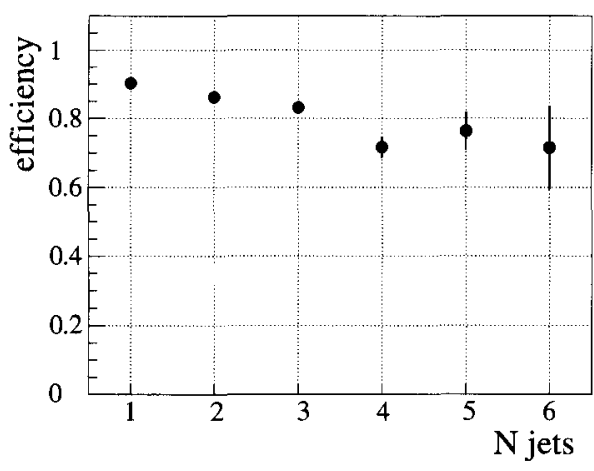

(a)

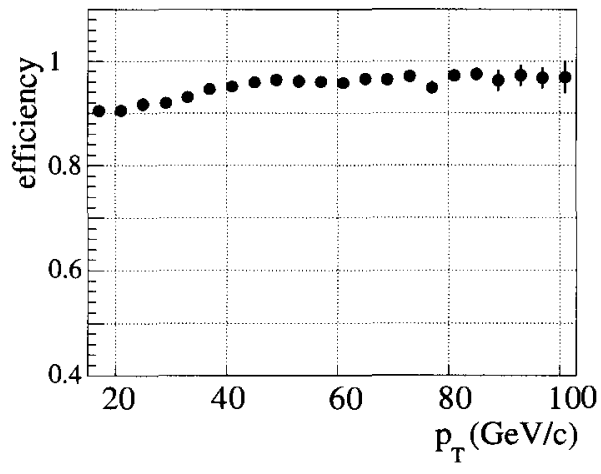

(b)

Figure 5.8: Isolation efficiency for $Z \rightarrow \mu \mu$ data as a function of (a) the number of jets with $E_{T}>15 \mathrm{GeV}$ and (b) muon $p_{T}$.

marginally passing the isolation cut, in the following way.

Figure 5.9(a) shows a fit (with the sum of two exponentials) to the invariant mass distribution of events in which both muons fail both isolation criteria. This is used to model the shape of the non-isolated background. It can be seen to fall more steeply with increasing dimuon mass than the Drell-Yan background.

The $Z$ peak shape is fitted on events where one muon passes both isolation criteria and one muon fails one isolation criterion (figure 5.9(b)). While the exponential background term has the same shape as for the non-isolated subsample, the $Z$ peak is wider and more shifted. (This is largely because of the $\sim 50 \%$ of these events for which the second muon fails the calorimeter isolation but passes the track isolation. These might be due to the second muon radiating off a photon, which means the reconstructed muon track will 'miss' some of the muon's energy. Figure $5.9(\mathrm{~d})$ shows the invariant mass distribution for both subsamples.)

Figure 5.9(c) shows the mass distribution of events where one muon fails both isolation criteria and the other passes them both, fitted with the sum of the background and signal functions. By extrapolation, it can be seen that the amount of non-isolated background in the signal regions $\left(M \gtrsim 300 \mathrm{GeV} / c^{2}\right)$ is negligible.

\subsubsection{Topology}

- $\Delta z<3 \mathrm{~cm}$

The distance between the tracks along the beam direction at the origin is required to be less than $3 \mathrm{~cm}$. This is a loose cut (cf. figure 5.10(a)) to make sure the muons are coming from the same vertex. Figure 5.11 shows the invariant mass of events failing exclusively this cut. The efficiency of this cut measured on $Z$ events is $99.5 \pm 0.4 \%$.

- $\Delta R(\phi, \eta)>0.1$ 


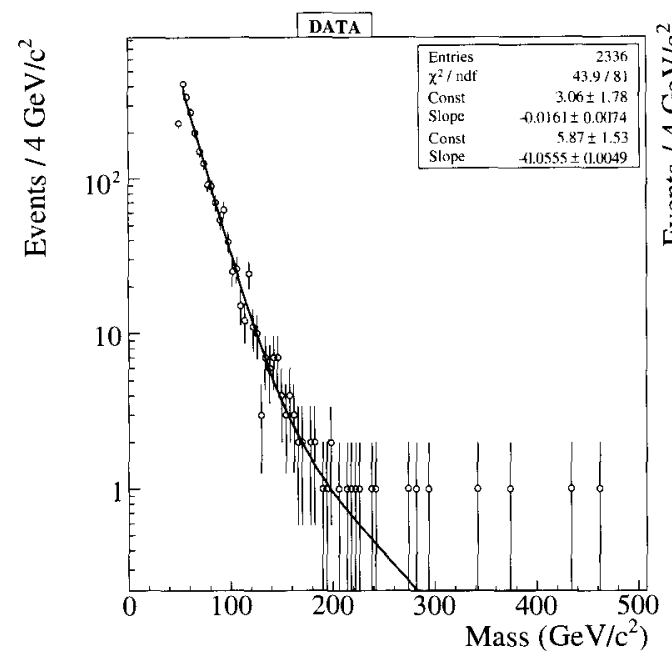

(a)

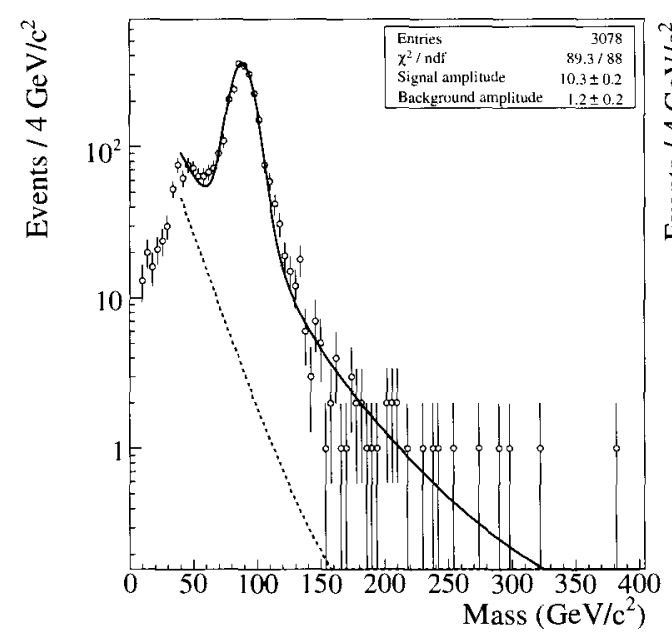

(c)

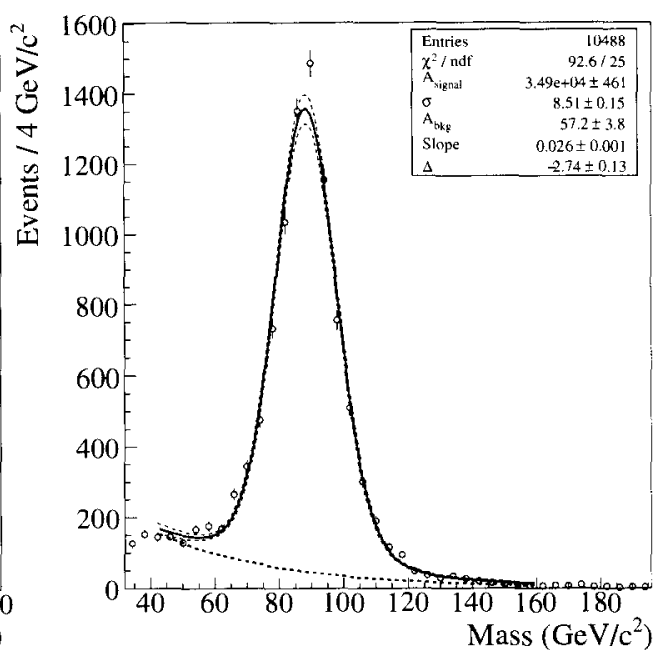

(b)

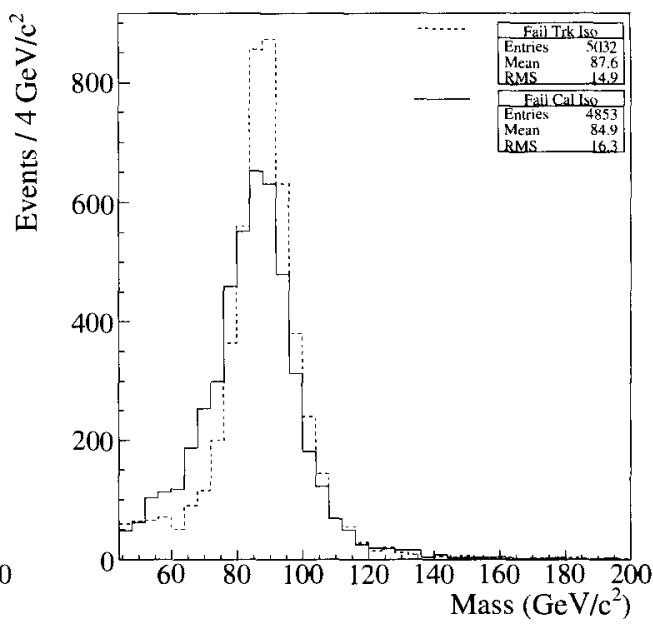

(d)

Figure 5.9: Invariant dimuon mass for events in which (a) both muons fail both the calorimeter and track isolation cut (these events are not included in the final selection); (b) one muon passes both isolation cuts and the other fails either the calorimeter or track isolation cut; (c) one muon passes both isolation cuts and the other fails both isolation cuts.

(d) shows the invariant mass for events as in (b) for which $M>40 \mathrm{GeV} / c^{2}$, separately for the events where the second muon fails the track isolation cut (dashed line) and the calorimeter isolation cut (solid line). (The events shown in (b), (c) and (d) are included in the selection.)

The dotted line in figure (c) shows the fit of figure (a), while the solid line is the peak plus background line of figure (b). 


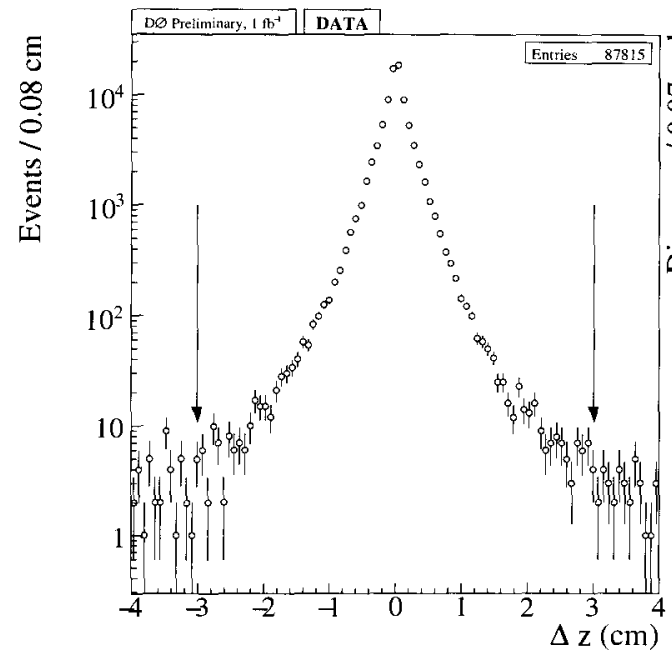

(a)

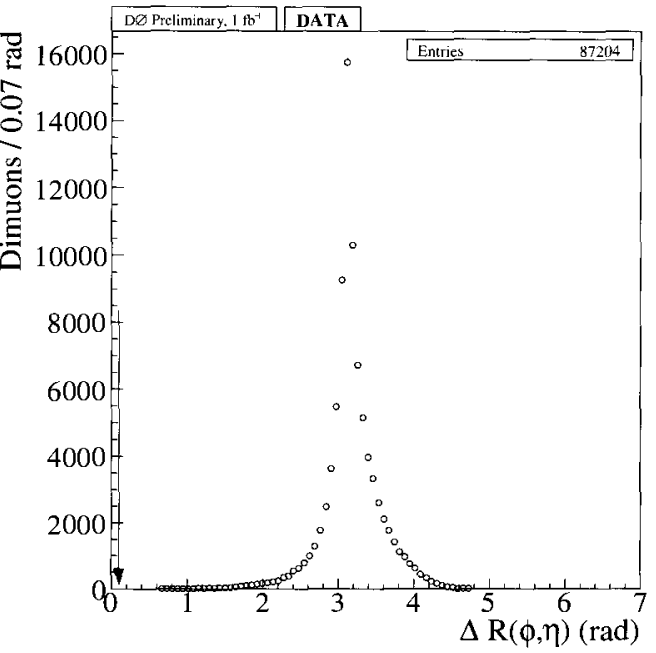

(b)

Figure 5.10: (a) $\Delta z$, after all other cuts have been applied. (b) $\Delta R(\phi, \eta)$, after all other cuts have been applied. Both for events with $M>40 \mathrm{GeV} / c^{2}$.

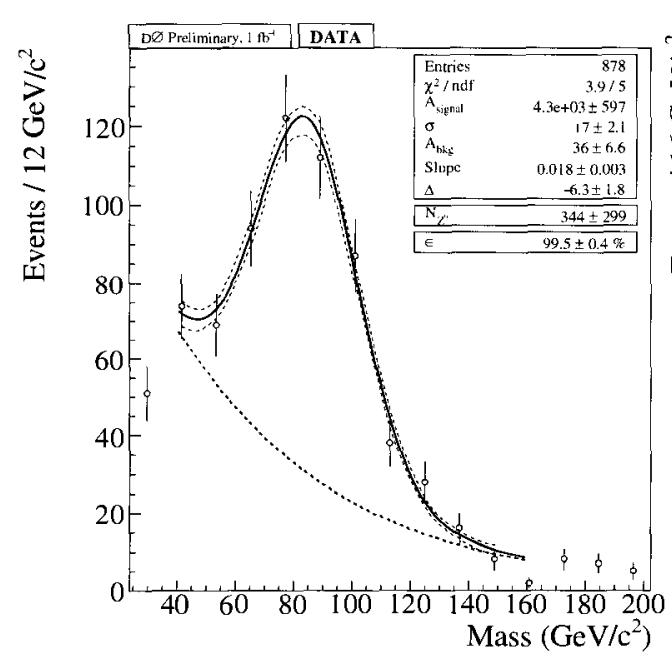

(a)

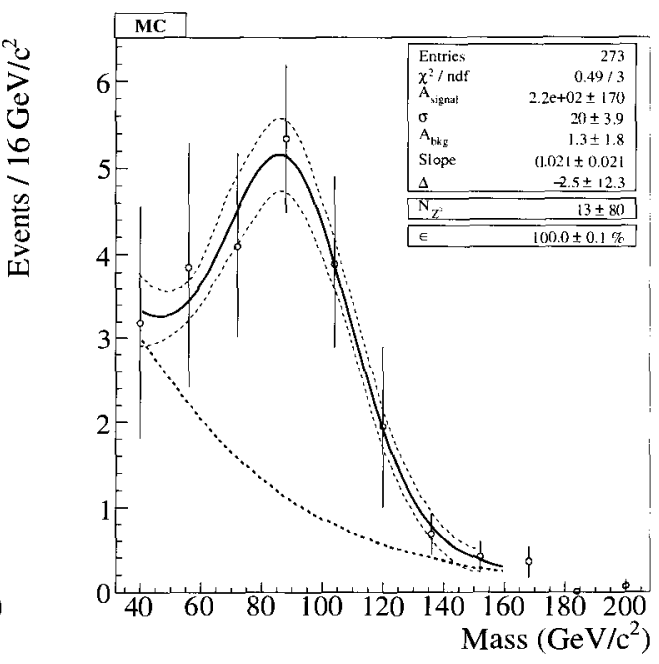

(b)

Figure 5.11: Dimuon mass for events exclusively failing the cut on $\Delta z$, data (a) and Monte Carlo (b). 


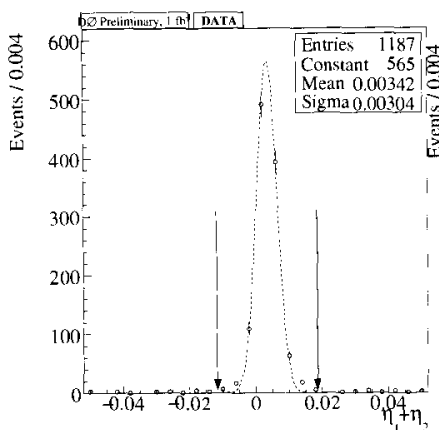

(a)

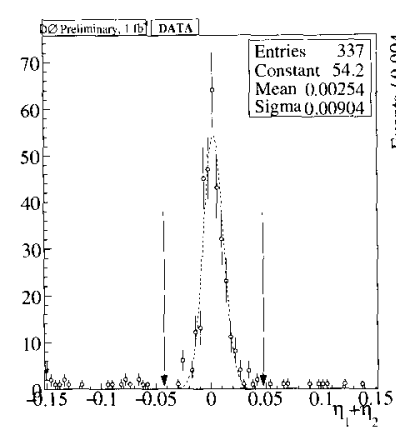

(b)

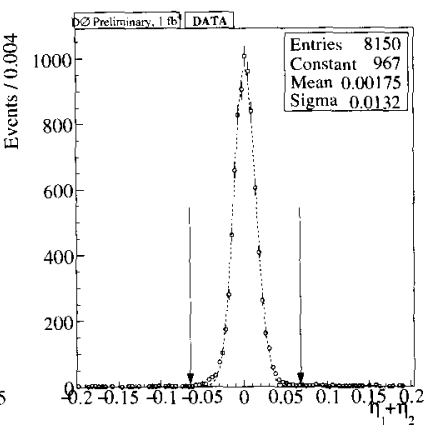

(c)

Figure 5.12: Sum of the pseudo-rapidity of the two muons for events exclusively failing the timing cuts, with respectively (a) both muons having at least one SMT hit, (b) one muon having at least one SMT hit and the other none, or (c) both muons having no SMT hits, respectively

This cut mainly removes events where one muon is reconstructed twice. It is almost completely correlated with a number of other cuts; in the 136298 events passing the muon ID, track and isolation cuts, 14552 dimuon pairs fail the $\Delta R$ cut, but only 6 events fail it exclusively.

The muon pairs failing the $\Delta R$ cut are mainly non-isolated ones, and/or occur in events where a good muon pair is accompanied by a third muon that is very close to one of the other two. Of course the dimuon invariant mass of pairs failing this cut is much smaller than $M_{Z}$, so in the end it merely serves to 'clean up' the complement samples of the other cuts, and does not remove any signal events nor changes the normalization.

\subsubsection{Cosmic muons}

Another background comes from muons originating from cosmic showers, where the same muon is reconstructed as two muon-matched tracks as it traverses the detector. A cut on the difference between the scintillator hit times of both muons, in each layer respectively, removes most cosmic events. However, some cosmic events can pass this cut, since the loose muon definition allows one or more scintillator misses. In order to be able to keep the more efficient loose muon definition but remove all cosmic events from the selection, a tight cut on the polar angle between the muons is applied in addition to the cuts on the timing.

Since for a cosmic event the same muon is reconstructed twice, the two reconstructed muon objects should be exactly back-to-back. Muon pairs produced by a Drell-Yan type process will generally be back to back in the transverse plane, but not in the longitudinal plane, due to the boost of the parton-parton system. Therefore, a cut on the polar angle between the muons will tend to remove cosmic events but not signal events. 


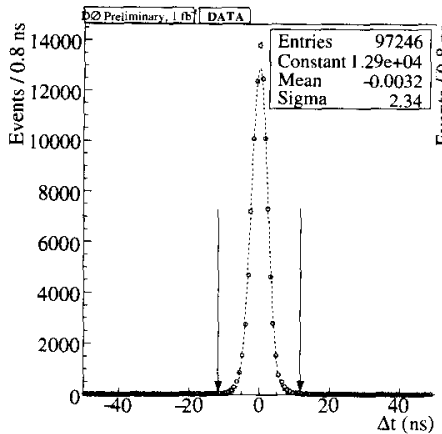

(a)

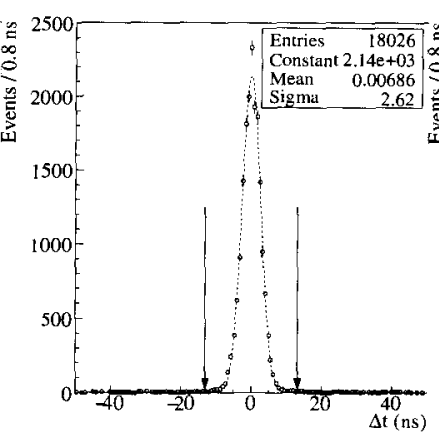

(b)

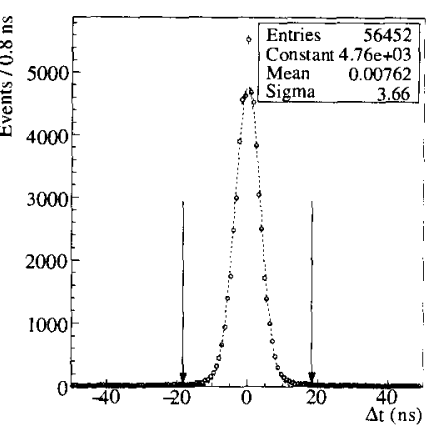

(c)

Figure 5.13: Time difference between (a) A-, (b) B- and (c) C-layer scintillator hits for events passing the cut on $\eta_{1}+\eta_{2}$ and where both muons have a scintillator hit in that layer.

Specifically, a Gaussian distribution is fitted to the distribution of the sum over the pseudo-rapidities of the two muons, for events failing one of the timing requirements. Events within $\pm 5 \sigma$ of this Gaussian are then rejected by this cut. Conversely, the timing cut is derived on events passing the cut on $\eta_{1}+\eta_{2}$ by fitting a Gaussian to the time difference between scintillator hits of each muon in the same layer, and events outside $\pm 5 \sigma$ are rejected. (This procedure is iterated between the two cuts, and the selection stabilizes after a few iterations.)

The cut on the polar angle is derived and applied separately for events with none, either or both of the muons having at least one hit in the silicon tracker respectively. The $\Delta t$ cuts are derived and applied independently for the difference between the times of scintillator hits in the $\mathrm{A}, \mathrm{B}$ and $\mathrm{C}$ layer, for events where both muons have a scintillator hit in that layer.

Figures 5.12 shows the $\eta_{1}+\eta_{2}$ distributions with the fits and cut values, while figures 5.13 shows the $\Delta t$ distributions with the fits and cut values.

In addition, each individual muon was required to pass the standard cosmic timing cut of $\pm 10 \mathrm{~ns}$ in the $\mathrm{A}$ - and BC-layer (as part of the loose muon quality requirement). This cut is almost completely redundant with the cut on the time difference. It is applied anyway because the muon ID and tracking efficiency corrections were derived for muons passing this cut. Figure 5.14 shows the invariant mass distribution for events failing only this cut; its efficiency for $Z$ events is $98.1 \pm 0.4 \%$.

Figure 5.15 shows the invariant mass distribution for events failing only the $\Delta t$ cuts; the efficiency of this cut for $Z$ events is consistent with $100 \%$. Figure 5.16 shows the invariant mass distribution for events failing only the $\eta_{1}+\eta_{2}$ cuts; the efficiency of this cut for $Z$ events is $99.0 \pm 0.3 \%$.

Figure 5.17 shows the invariant mass distribution of events failing only (all) the anticosmic cuts; no $Z$ peak can be found in this sample. 


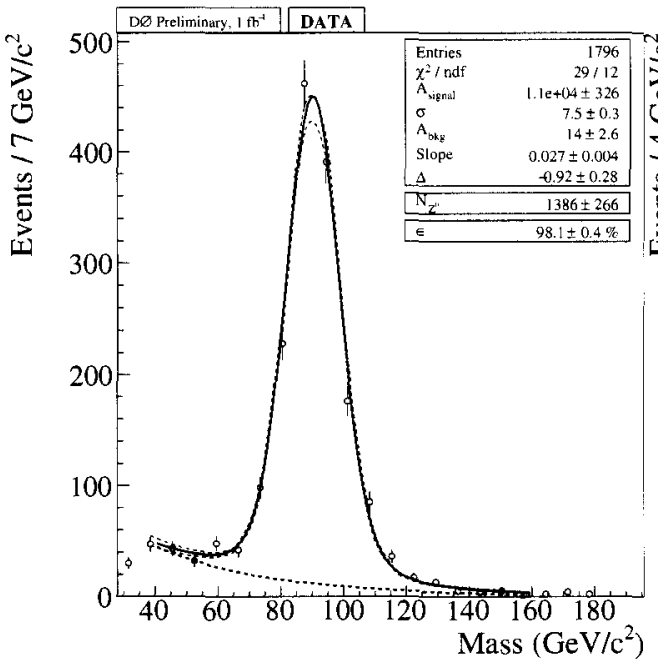

(a) Data

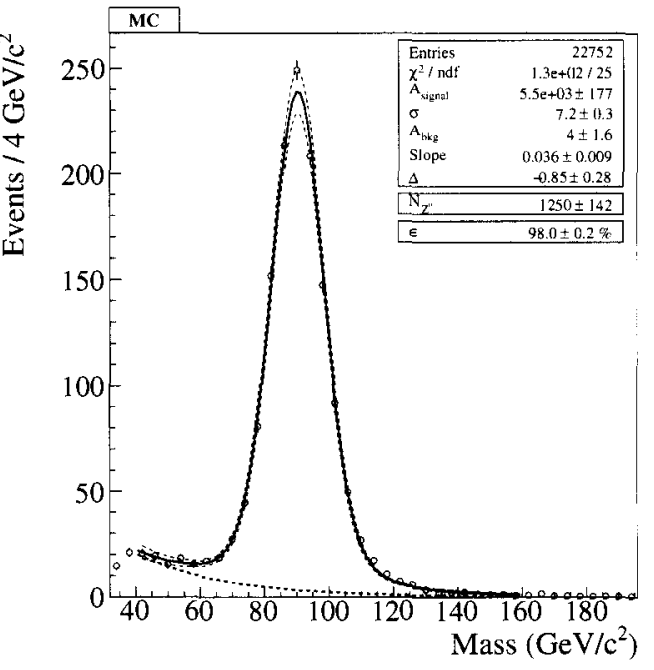

(b) Monte Carlo

Figure 5.14: Dimuon mass for events exclusively failing the cut on the individual muon scintillator hit times.

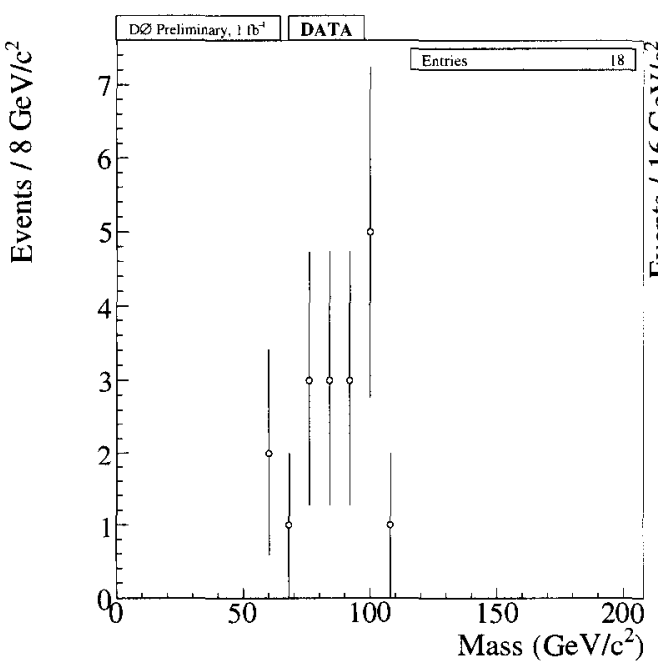

(a) Data

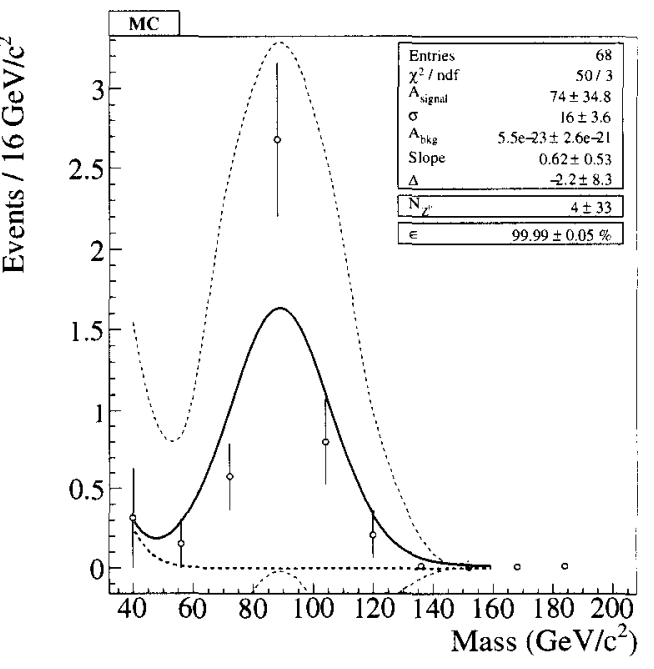

(b) Monte Carlo

Figure 5.15: Dimuon mass for events exclusively failing $\Delta t$ cut, with both muons passing cuts on the individual muon scintillator hit times (scintillator hit times within \pm 10 ns of beam crossing time). 


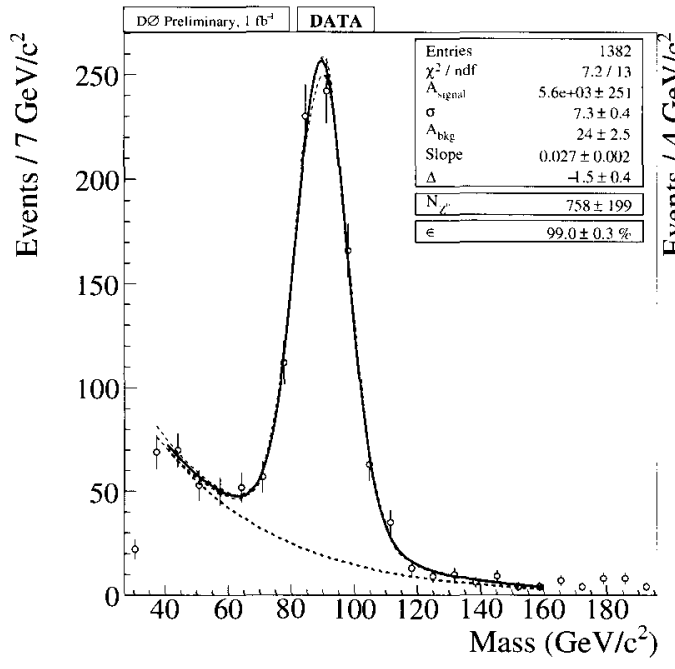

(a) Data

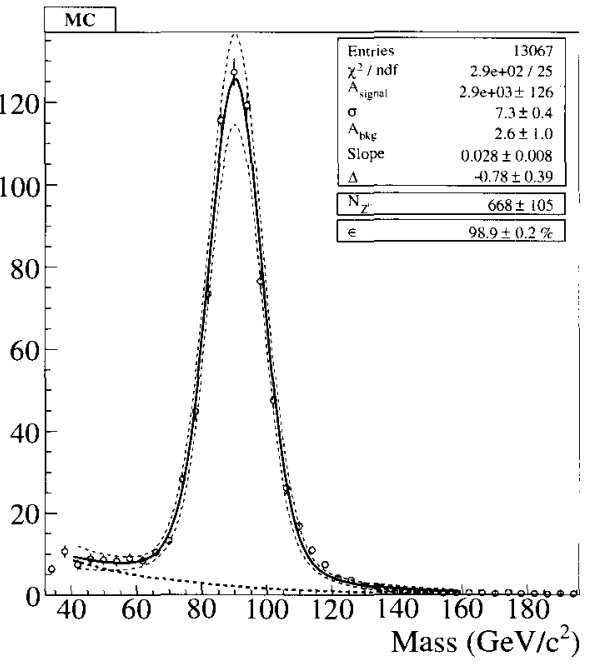

(b) Monte Carlo

Figure 5.16: Dimuon mass for events exclusively failing $\eta_{1}+\eta_{2}$ cut.

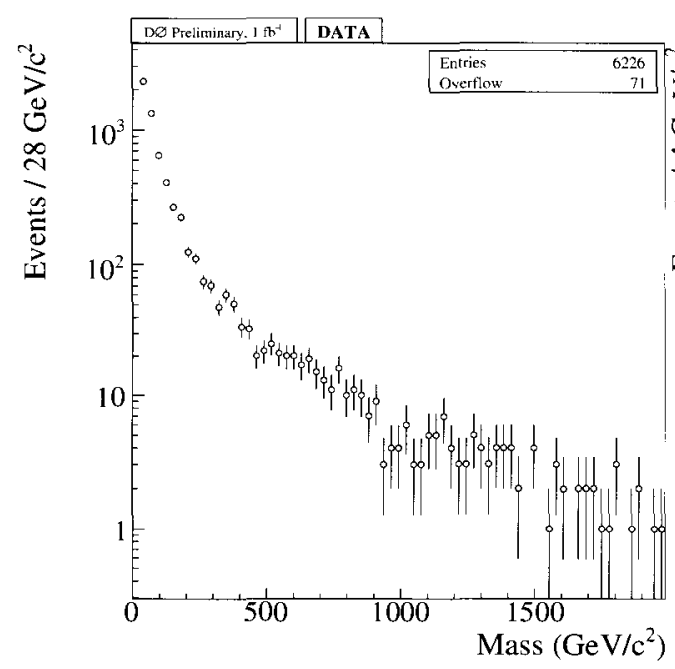

(a)

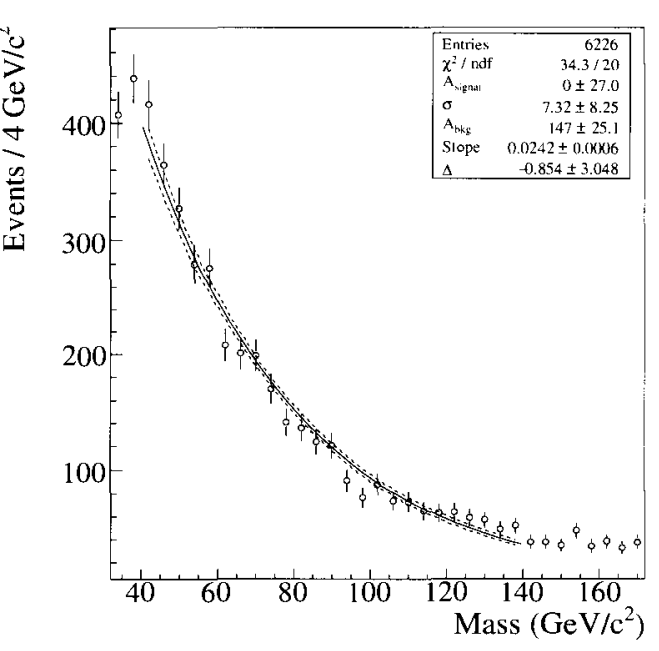

(b)

Figure 5.17: Dimuon mass, events exclusively failing both the timing and $\eta_{1}+\eta_{2}$ cuts. (b) shows the region around the $Z$ peak. 


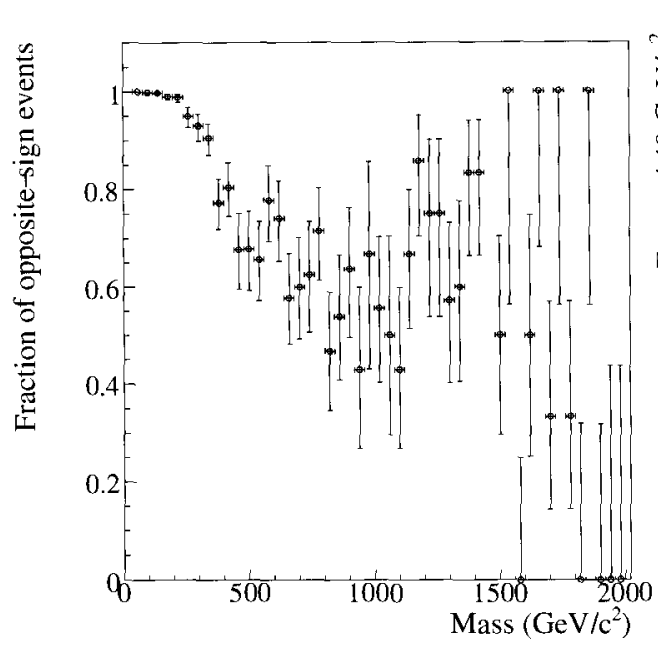

(a)

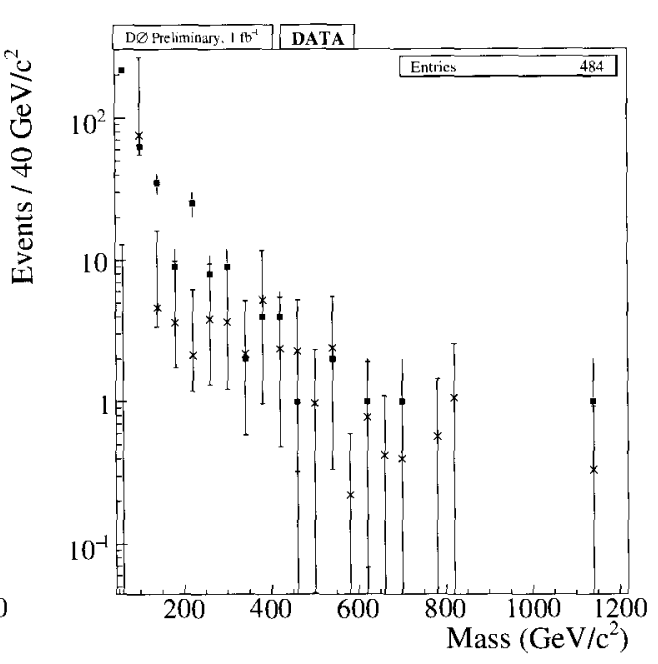

(b)

Figure 5.18: (a) Fraction of events with opposite sign muons in a given mass bin, for events failing exclusively anti-cosmic cuts,

(b) Round markers: invariant mass distribution of events in final selected sample with like-sign muons; crosses: fraction of like-sign cosmic events times selected events, per bin.

\subsubsection{Charge}

While the signal event final state is expected to contain two oppositely charged muons, no cut on the charge of the muon is in fact applied. On the one hand, the expected remaining Standard Model like-sign background cross section is negligible at higher invariant dimuon mass relatively to the Drell-Yan cross section, while on the other hand the probability that the charge of the muon is wrongly reconstructed increases with the $p_{T}$ of the muon. To assess the efficiency of a possible charge cut on the selected events, one can look at the fraction of cosmic events (failing the $\left|\eta_{1}+\eta_{2}\right|$ and scintillator timing cuts, see figure 5.17) that are opposite sign, as a function of the reconstructed dimuon mass. Since these muon objects are actually likely to be a single muon from a cosmic shower reconstructed twice, their charge should be opposite from each other. From figure 5.18(a), it can be seen that the fraction of correctly measured track curvatures drops for a reconstructed dimuon mass above $\sim 200 \mathrm{GeV} / c^{2}$.

The dimuon charge reconstruction efficiency for cosmic muons can be used to obtain an estimate of the expected number of like-sign events in the final sample, assuming that all cosmic events are in fact opposite-sign events. Figure 5.18(b) shows the invariant mass distribution of like-sign muon pairs in the final selected sample (round markers) as well as, per bin, the fraction of like-sign events in the cosmic sample times the number of selected events in that bin (crosses). These can be seen to (roughly) agree at high reconstructed dimuon mass. Of course, the charge reconstruction efficiency is possibly less good for (out-of-time) cosmic events than for events in the final selection, 
which would mean that the number of expected like-sign events in the final selection should in fact be lower.

The total number of selected like-sign events with $M>80 \mathrm{GeV} / c^{2}$ is $165 \pm 13$ whereas the estimated expected number is $216_{-90}^{+4732}$.

\subsubsection{More than 2 muons}

In the case that an event has more than one dimuon pair (i.e., combination of two muons) which passes all the cuts, the two 'most isolated' muons are selected, i.e., the two muons for which the sum of the track halo and calorimeter halo (see section 5.4.3) is the smallest. This occurs in 45 events in the final sample, out of which 18 have two muon pairs passing all cuts (most of those have two reconstructed muons which do not pass the $\Delta R$ cut together and are likely to be one muon that was reconstructed twice), and 26 events have exactly 3 muon pairs passing all cuts (of which 24 have 3 muons passing all cuts).

Assuming this last signature comes from $\gamma^{\star} / Z \rightarrow \mu^{+} \mu^{-}$decays where a third muon comes from a heavy quark jet, the number of such events in the selected sample gives an upper limit for the expected number of selected $W \rightarrow \mu \nu$ events where the second muon comes from a heavy quark jet. Assuming that $>0$-jet branching fraction is the same for $Z$ 's as for $W$ 's and neglecting muon selection efficiency correlations,

$$
N(W) \lesssim \frac{\sigma(W \rightarrow \mu \nu)}{\sigma\left(Z \rightarrow \mu^{+} \mu^{-}\right)} N_{>1 \mu \text { pair }} / \mathrm{P}_{2^{\text {nd }} \mu}=\frac{2.416}{0.2416} \cdot 26 / 0.56=464 \pm 91 \text { (stat.) }
$$

where $\mathrm{P}_{2^{\text {nd }} \mu}$ is the conditional probability for the second muon in a $Z \rightarrow \mu^{+} \mu^{-}$event to be identified (calculated on the $\gamma^{*} / Z$ Monte Carlo sample).

For comparison, the expected number of selected $W$ events using the Monte Carlo simulation is 263. Since this background is difficult to predict theoretically, such a discrepancy is not unexpected. In either case, this background is expected to be small.

\subsubsection{Final sample}

The final data sample contains 94160 events, which is $1.2 \%$ of the number of events passing the data quality requirements. Table 5.4 shows the number of events passing each consecutive cut and complement sample size for cuts in the the final event selection. Figure 5.19 shows the reconstructed invariant mass for all dimuon pairs in selected events with a mass around the $Z$ peak. As in previous plots, the distribution is fitted around the $Z$ peak with the generator-level Breit-Wigner plus an exponential background term, convoluted with a non-central Gaussian. In the limit setting, the Monte Carlo normalization will be determined using the result of the kinematic fit, described below.

\footnotetext{
${ }^{2}$ Here and in figure 5.18(a) the statistical error corresponds with the shortest $68.3 \%$ Bayesian confidence interval given that the fraction of like-sign events has to lie between 0 and 1; see [84] for details.
} 


\begin{tabular}{|lrrr|}
\hline Selection & N passing & \% of prev. & \% of total \\
\hline Initial & 9741062 & & \\
Remove bad runs and lbns & 8348914 & $85.71 \pm 0.01$ & $85.71 \pm 0.01$ \\
Event quality cuts & 7866831 & $94.23 \pm 0.01$ & $80.76 \pm 0.01$ \\
Single- or dimuon trigger & 5087418 & $64.67 \pm 0.02$ & $52.23 \pm 0.02$ \\
\hline Muon out of bottom hole & 5076839 & $99.79 \pm 0.00$ & $52.12 \pm 0.02$ \\
Muon detector $|\eta|<2$ & 5073216 & $99.93 \pm 0.00$ & $52.08 \pm 0.02$ \\
Loose quality muon & 5005377 & $98.66 \pm 0.01$ & $51.38 \pm 0.02$ \\
Muon matched with central track & 4980138 & $99.50 \pm 0.00$ & $51.13 \pm 0.02$ \\
$\mid$ dca $\mid<0.02$ (SMT), 0.2 cm (no SMT) & 3751288 & $75.32 \pm 0.02$ & $38.51 \pm 0.02$ \\
(corrected) muon $p_{T} \geq 15$ GeV $/ c$ & 2886218 & $76.94 \pm 0.02$ & $29.63 \pm 0.01$ \\
\hline $2^{\text {nd } m u o n ~ o u t ~ o f ~ b o t t o m ~ h o l e ~}$ & 2708814 & $93.85 \pm 0.01$ & $27.42 \pm 0.01$ \\
$2^{\text {nd } m u o n ~ d e t e c t o r ~}|\eta|<2$ & 2676184 & $98.80 \pm 0.01$ & $27.09 \pm 0.01$ \\
$2^{\text {nd }}$ muon $L o o s e$ quality & 1516259 & $56.66 \pm 0.03$ & $15.35 \pm 0.01$ \\
$2^{\text {nd } m u o n ~ m a t c h e d ~ w i t h ~ c e n t r a l ~ t r a c k ~}$ & 1347656 & $88.88 \pm 0.03$ & $13.64 \pm 0.01$ \\
$2^{\text {nd }}$ muon passing DCA cuts & 1088776 & $80.79 \pm 0.03$ & $11.02 \pm 0.01$ \\
$2^{\text {nd }}$ muon $p_{T} \geq 15$ GeV $/ c$ & 136298 & $12.52 \pm 0.01$ & $1.40 \pm 0.00$ \\
\hline Fail at most one of the following cuts & 119302 & $87.53 \pm 0.09$ & $1.22 \pm 0.00$ \\
$\geq 6$ CFT hits & 118248 & $99.12 \pm 0.03$ & $1.21 \pm 0.00$ \\
$\Delta R(\phi, \eta)>0.1$ rad & 118242 & $99.99 \pm 0.00$ & $1.21 \pm 0.00$ \\
Track $\Delta z>3$ cm & 117382 & $99.27 \pm 0.02$ & $1.21 \pm 0.00$ \\
at least one isolated muon & 106792 & $90.98 \pm 0.08$ & $1.10 \pm 0.00$ \\
anti-cosmic cuts & $\mathbf{9 4 1 6 0}$ & $88.17 \pm 0.10$ & $0.97 \pm 0.00$ \\
\hline
\end{tabular}

Table 5.4: The number of events passing each cut, starting from the 2MUhighpt skim. The last partition shows the final dimuon selection cuts; 119302 events fail at most one of these cuts; 118248 of those pass the nCFT cut, 118242 of those pass the $\Delta R$ cut as well; etc. The final selected sample passing all cuts contains 94160 events. 


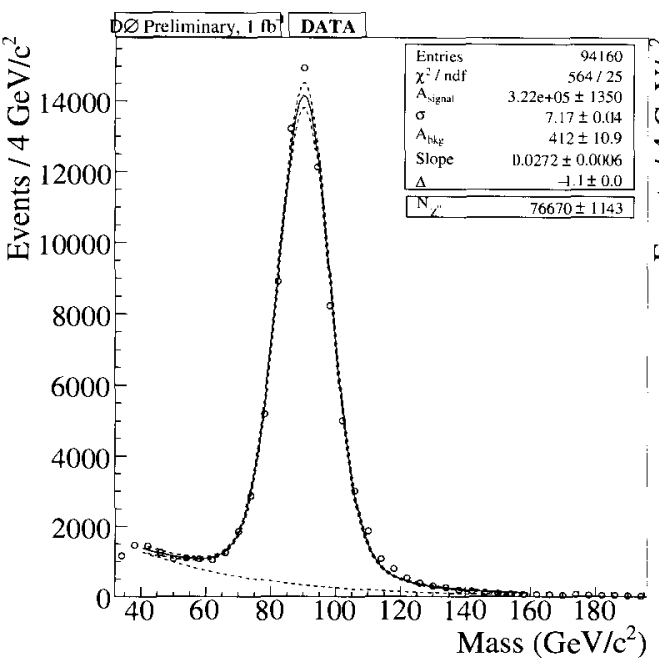

(a)

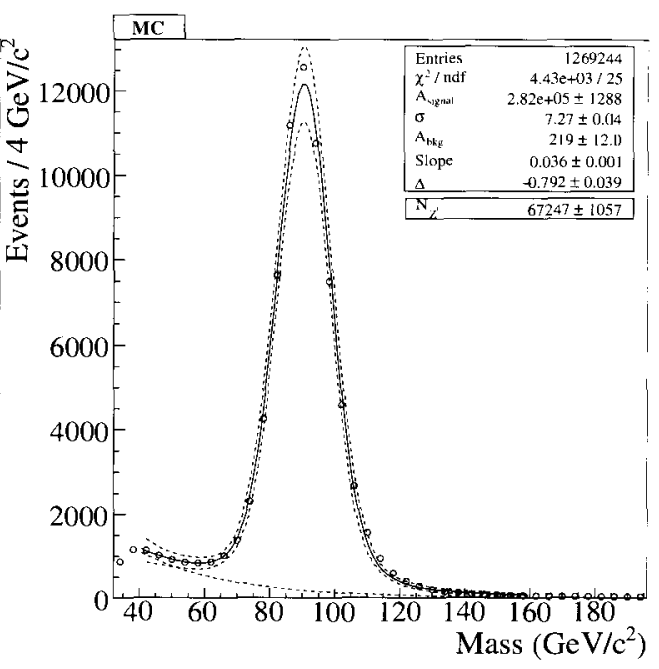

(b)

Figure 5.19: Reconstructed dimuon mass for events in final selection, data (a) and Monte Carlo (b).

\subsection{Kinematic fit}

In the final sample, the predominant remaining background in the signal regions $\left(M_{\mu \mu} \gtrsim 300 \mathrm{GeV} / c^{2}\right)$ comes from $Z \rightarrow \mu^{+} \mu^{-}$events where one or both muons has a central track that has been reconstructed with a too high transverse momentum. This is caused by the fact that the track resolution is approximately Gaussian in $\frac{1}{p_{T}}$ rather than in $p_{T}$ while the cross section drops exponentially at invariant masses above the $Z$ mass, in addition to possible non-Gaussian effects.

The total transverse energy in the event is expected to add up to zero. The leading order contribution to the hard scatter has two muons that are almost exactly back-toback with little hadronic recoil in the event, but higher order corrections can lead to events where the dimuon system has a non-zero transverse $p_{T}$ (more in section 2.2).

The track momenta measurements are improved by a kinematic fit against the total recoil energy of the vector boson, as measured by the calorimeter. The reconstructed muon track polar angles are kept fixed in the fit, as little improvement over the track angle measurement can be expected.

Specifically, for each event the following function is minimized over the fitted muon using the MIGRAD method in MINUIT:

$$
\chi^{2}\left(\vec{p}_{T 1}, \vec{p}_{T 2}\right)=\left[\sum_{i=1,2}\left(\frac{1 / p_{T 1 i}^{\text {(reco) }}-1 / p_{T_{i}}}{\Delta\left(1 / p_{T i}^{\text {(reco) }}\right)}\right)^{2}\right]+\left(\frac{p_{T}^{(Z)}\left(\not_{T}\right)-\left|\vec{p}_{T 1}+\vec{p}_{T 2}\right|}{\Delta p_{T}^{(Z)}\left(\mathscr{E}_{T}\right)}\right)^{2}
$$

Here, 


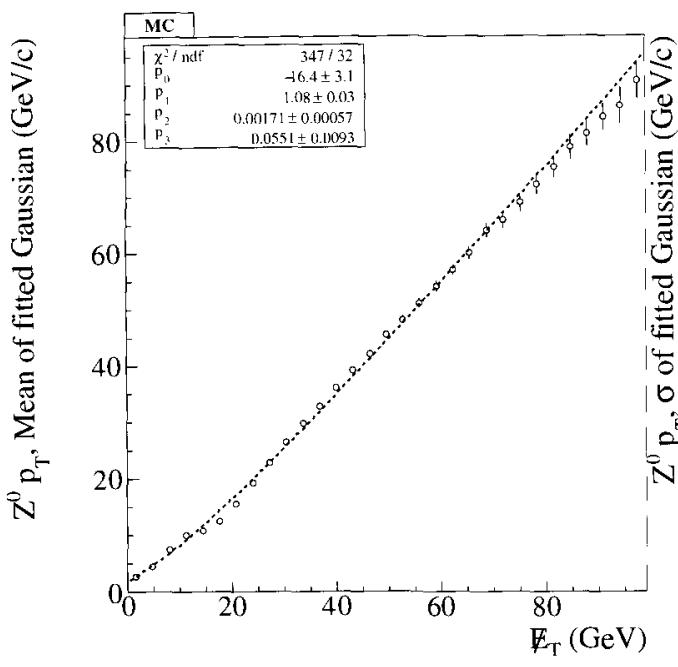

(a)

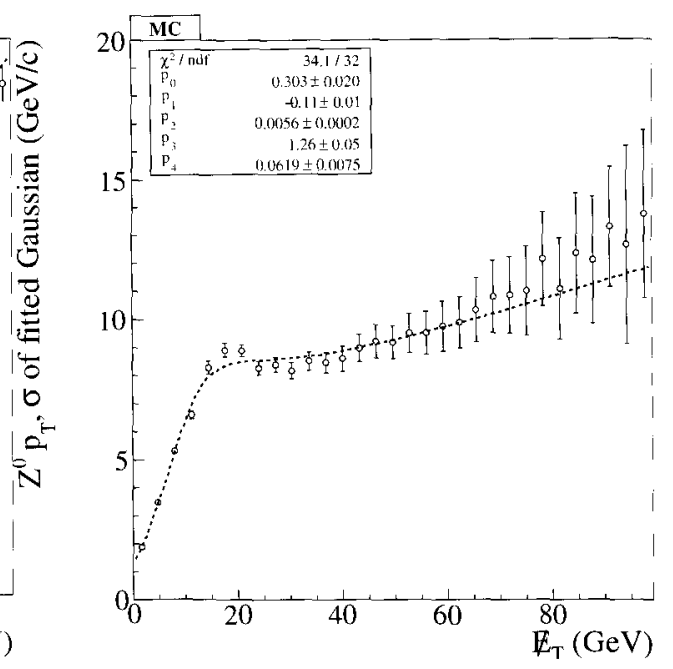

(b)

Figure 5.20: (a) Mean and (b) sigma of a Gaussian fit to the $Z p_{T}$ distribution per $3.2 \mathrm{GeV}$ bin in the calorimeter missing transverse energy, fitted with the function in equations (5.4) and (5.5) respectively, $\left(\gamma^{\star} / Z\right.$ Monte Carlo).

- $p_{T i}^{(\text {reco) }}$ is the reconstructed track $p_{T}$ of the $i$-th muon,

- $\vec{p}_{T 1}+\vec{p}_{T 2}$ denotes the vector sum over the vectors with length $p_{T 1,2}$ in the direction of the reconstructed muon $\vec{p}_{T 1,2}$,

- $\Delta\left(1 / p_{T i}^{(\text {reco) }}\right)$ is the resolution on $1 / p_{T i}^{(\text {reco) }}$ as a function of $1 / p_{T i}^{(\text {reco) }}$,

- $p_{T}^{(Z)}\left(E_{T}\right)$ is the $p_{T}$ of the vector boson as a function of the missing transverse energy

- $\Delta p_{T}^{(Z)}\left(E_{T}\right)$ is the resolution on this quantity, as a function of the missing transverse energy

\subsubsection{Recoil parameterization}

To estimate the recoil, the transverse calorimeter energy in the event is measured by adding vectorially the energy in calorimeter cells. Energy deposited in cells in the coarse hadronic $(\mathrm{CH})$ layer of the calorimeter is not added, because energy in those cells is mostly from noise. An exception is made if a CH cell overlaps with a "good" reconstructed jet ${ }^{3}$. The calorimeter cell energy is corrected for the electromagnetic energy scale for cells which are part of reconstructed photons and electrons, and for

\footnotetext{
3"good" /"bad": jet ID criteria aimed at removing jets that are due to noise, "hot cells" etc., and reflect the noise characteristics and geometry of the detector. For details of jet reconstruction, jet quality and correction, see [85]
} 
the jet energy scale for cells in "good" jets. "Bad" jets are removed and their energy is not added to the total.

To account for the energy loss of the muons, the energy deposited in a 0.1 cone in $R(\phi, \eta)$ around each muon was subtracted.

The correlation of the corrected missing transverse energy in the calorimeter, $E_{T}$, with the true (generator-level) vector boson transverse momentum $p_{T}^{(Z)}$ is derived for the same Monte-Carlo samples that are used to simulate the $\gamma^{\star} / Z$ background. These have zero-bias overlay and are reweighted to match the $Z p_{T}$ distribution in $Z \rightarrow e^{+} e^{-}$data (see section 4.2). The dependence on the missing transverse energy of the mean $p_{T}^{(Z)}\left(E_{T}\right)$ and one standard deviation width $\Delta p_{T}^{(Z)}\left(E_{T}\right)$ is parameterized with suitably chosen functions, namely,

$$
\begin{gathered}
p_{T}^{(Z)}\left(\not_{T}\right)=p_{0}+p_{1} \mathbb{E}_{T}+\left(p_{2} \cdot E_{T}+p_{3}\right)^{-1} \\
\Delta p_{T}^{(Z)}\left(E_{T}\right)=\frac{p_{0} E_{T}}{\sqrt{1+p_{1} E_{T}+p_{2} \bar{E}_{T}^{2}}}+p_{3}+p_{4} \cdot E_{T}
\end{gathered}
$$

which are fitted to the mean and width of Gaussian distributions fitted to the $p_{T}^{(Z)}$ distribution for each $3.2 \mathrm{GeV}$ bin in $E_{T}$, as shown in figure 5.20 .

\subsubsection{Track resolution parameterization}

The track $p_{T}$-resolution as reproduced by the 'smeared' simulation was parameterized as a function of $p_{T}$. Different parameterizations were used for muons without SMT hits, central muons with SMT hits and forward/backward muons with SMT hits, and for each of these again for Monte Carlo smeared to match the pre-shutdown and postshutdown data (see section 5.3). Figure 5.25 on page 92 (at the end of this chapter) shows the used parameterizations.

\subsubsection{Results of the kinematic fit}

Figure 5.26 on page 93 shows the kinematic fit pull distributions on $\gamma^{\star} / Z$ Monte Carlo. From the fact that these distributions are close to being normal, it can be inferred that the uncertainties were reasonably well modeled and that the constraint is close to being Gaussian.

Figures 5.27 show the difference of the true (generated) vector boson mass with the reconstructed mass and with the fitted mass, respectively, again for $\gamma^{\star} / Z$ Monte Carlo. It can be seen that the number of events with a severely overestimated dimuon mass is reduced significantly after the fit.

In order to compare the fit pull on data and on Monte Carlo, we can compare the distribution of the following quantity,

$$
\frac{p_{T, \text { reco }}-p_{T, \text { fit }}}{\sqrt{\left|\sigma_{\text {fit }}^{2}-\Delta^{2}\left(p_{T}\right)\right|}}
$$




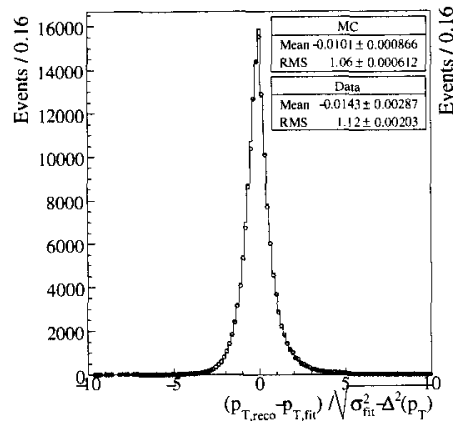

(a)

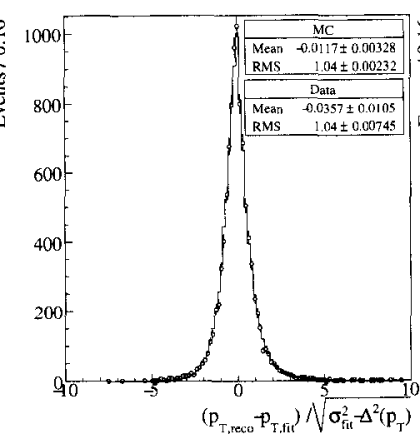

(b)

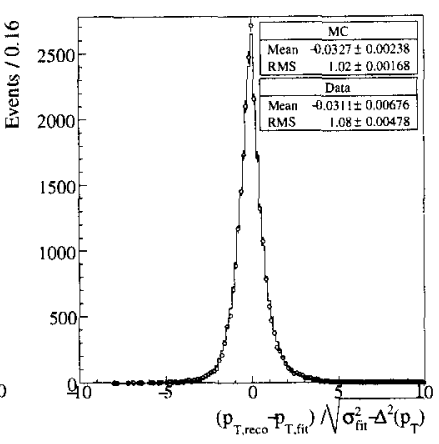

(c)

Figure 5.21: Pull difference distributions for central (a,d) and forward (b, e) muons with SMT hits, and muons without SMT hits (c, f). Data (open circles) and Standard Model Monte Carlo (line) with muon resolutions 'smeared' to match pre-shutdown (a-c) and post-shutdown (d-f) data.

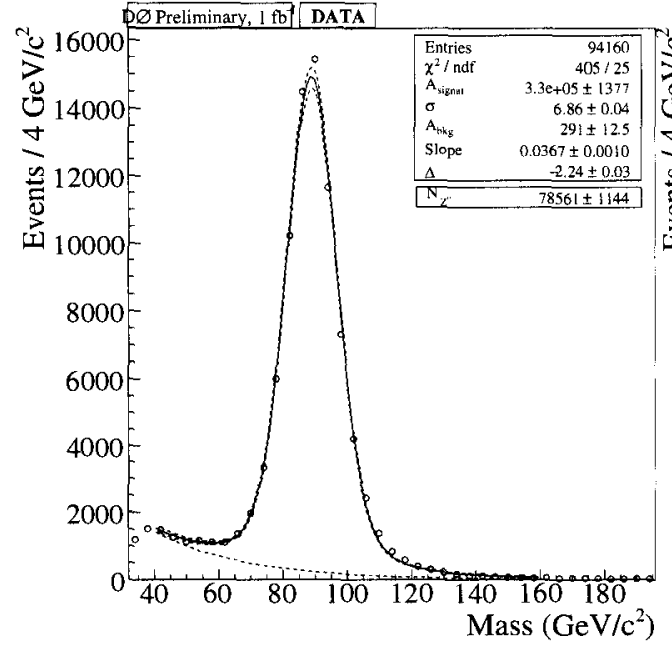

(a)

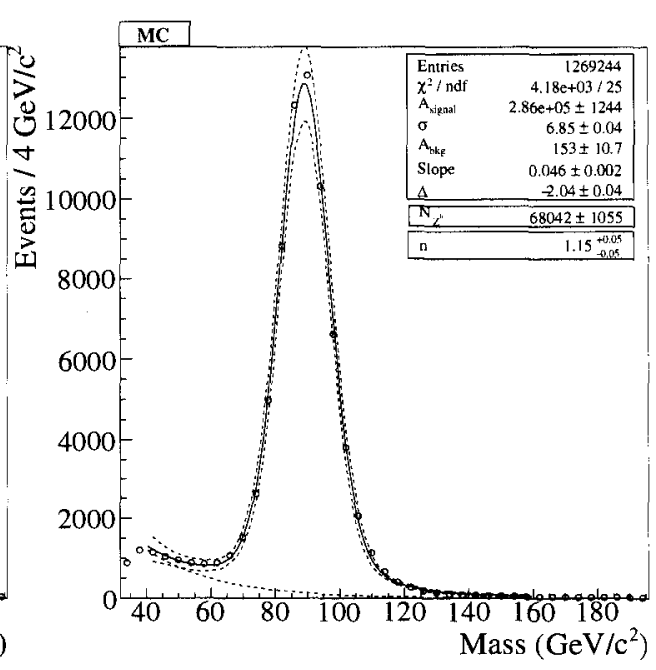

(b)

Figure 5.22: Binned likelihood fit on the dimuon $Z$ resonance for events after final selection, after the kinematic fit, for data (a) and Monte Carlo (b). 


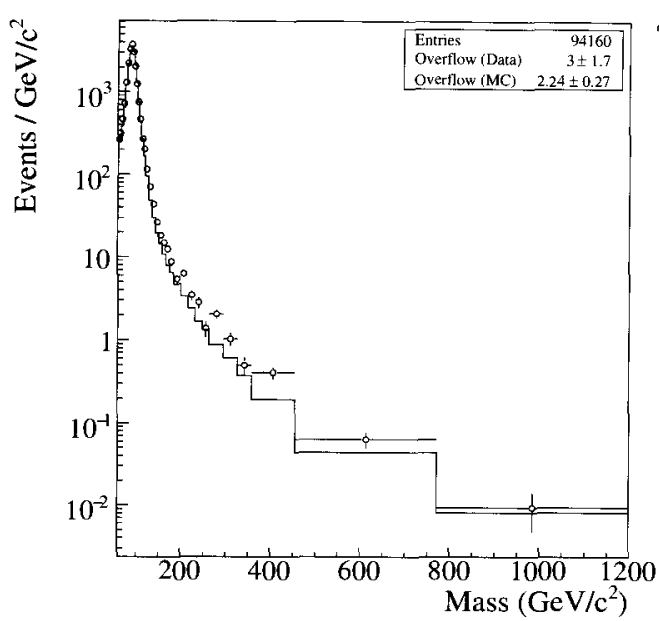

(a)

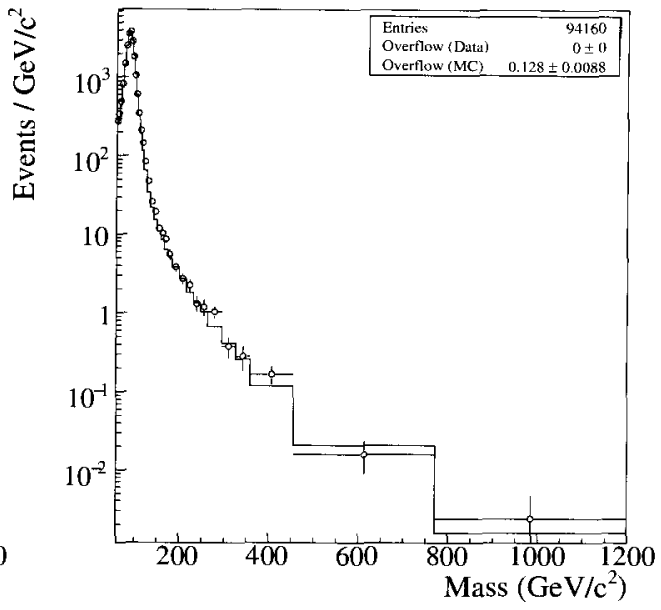

(b)

Figure 5.23: Dimuon mass for events in final selection before (a) and after (b) the kinematic fit, data (open circles) and Monte Carlo (line).

where $p_{T, \text { fit } / \text { reco }}$ are the measured muon $p_{T}$ before and after the fit respectively, $\sigma_{\text {fit }}$ is the fit error and $\Delta\left(p_{T}\right)$ is the track $p_{T}$ resolution. Figures 5.21 on the previous page show the distributions in data and Monte Carlo; they can be seen to agree quite well.

Figures 5.22 show a binned likelihood fit to the $Z$ peak for data and Monte-Carlo after the kinematic fit. The number of counted $Z$ decays and normalization are indicated as well. It can be seen that the overall mass resolution at the $Z$ peak is slightly improved. In addition, the number of $Z$ decays as counted with the fit is slightly increased after the fit as well.

There is a small difference of $1.25 \%$ between the Monte Carlo normalization before and after the fit, which is likely due to remaining differences in the track and calorimeter energy resolutions in data and Monte Carlo. This is added as an additional systematic uncertainty on the background prediction (see section 5.4.1).

Finally, figure 5.23 shows the full mass distribution after the fit. As expected, the (absolute) agreement between data and Monte Carlo is much better after the fit, because the dependence on the knowledge of the track resolution is reduced. Accordingly, the systematic uncertainty on the Standard Model background due to the track resolution uncertainty is also reduced (see figure 5.4(b)).

Figure 5.24 shows the ratio of data events over the expected number of background events, before and after the fit, with the systematic uncertainties on the expected background taken into account (for a description of the systematic uncertainties, see section 6.2.2). The difference between data and prediction in the $280 \mathrm{GeV} / c^{2}$ bin is $1.5 \sigma$ (stat. + syst.) after the fit. 


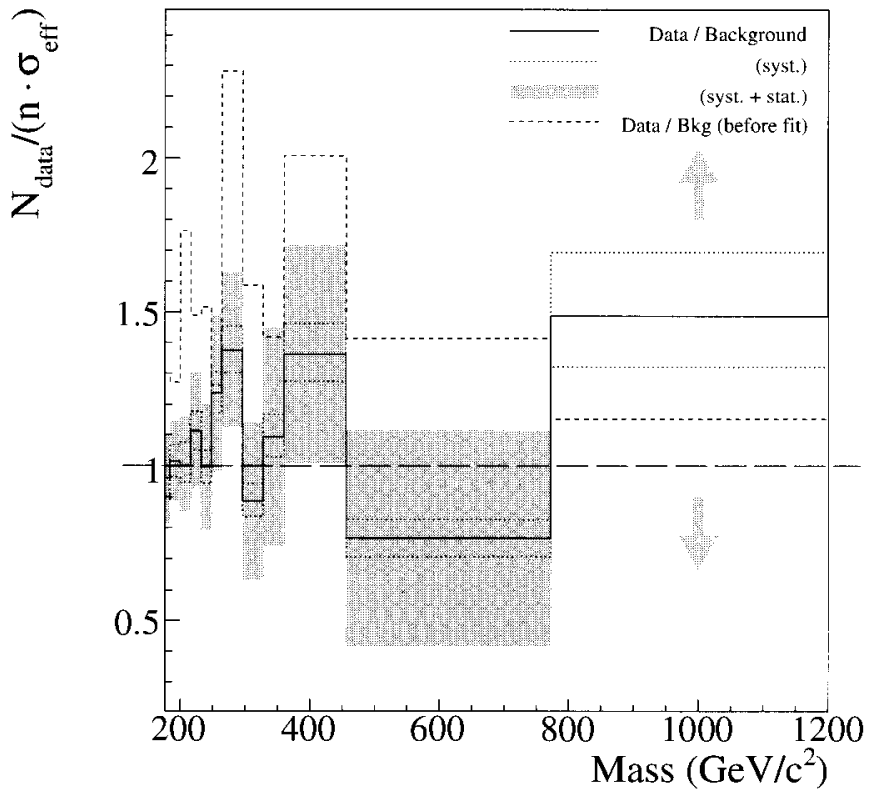

Figure 5.24: Ratio of the number of data events to the number of background Monte Carlo events in a bin, as a function of the (reconstructed or fitted) dimuon invariant mass. The dashed line solid line shows the result before the fit, the solid line the result after the kinematic fit. The gray band represents the systematic plus statistical uncertainty on the ratio (the uncertainty on the last bin exceeds the axis limits). 


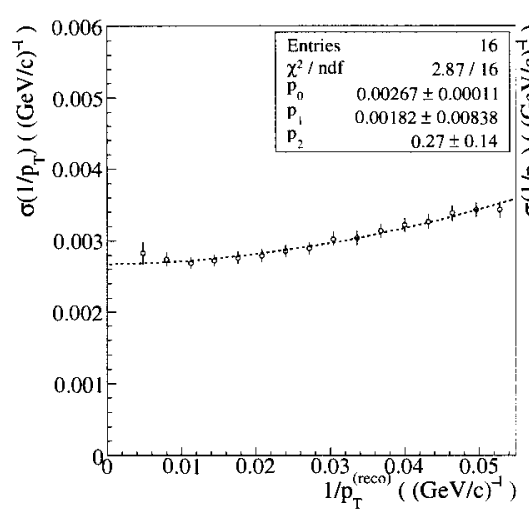

(a)

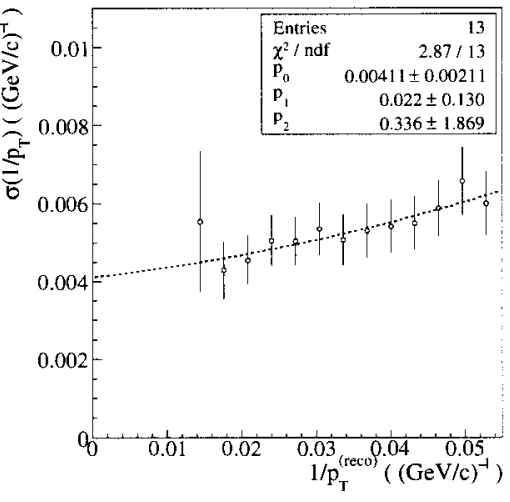

(b)

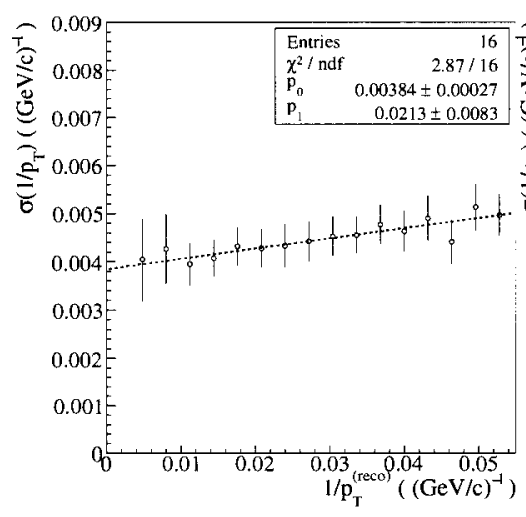

(c)

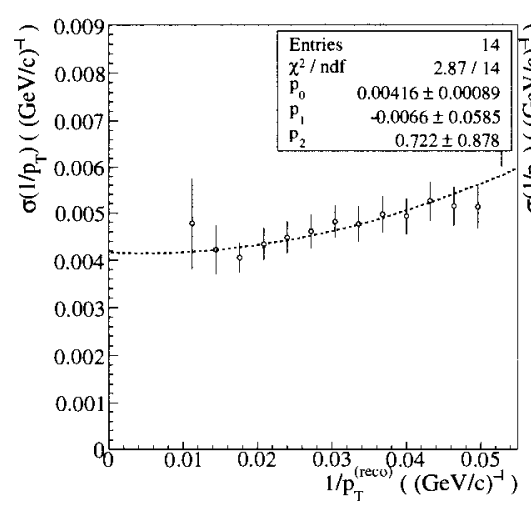

(e)

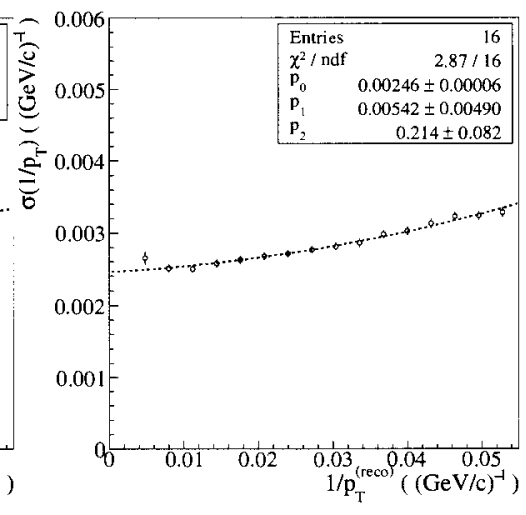

(d)

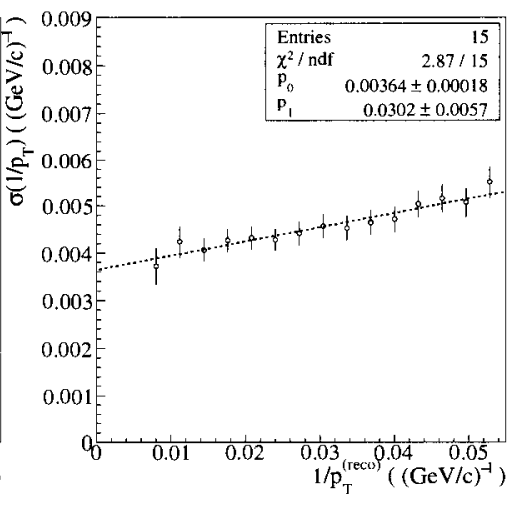

(f)

Figure 5.25: Sigma of a Gaussian fit to the true muon $1 / p_{T}$ per bin in the central track reconstructed $1 / p_{T}$ for central (a, d) and forward (b, e) muons with SMT hits, and muons without SMT hits (c,f). The plots are for Monte Carlo events with the muon resolutions 'smeared' to match pre-shutdown (a-c) and post-shutdown (d-f) data. 


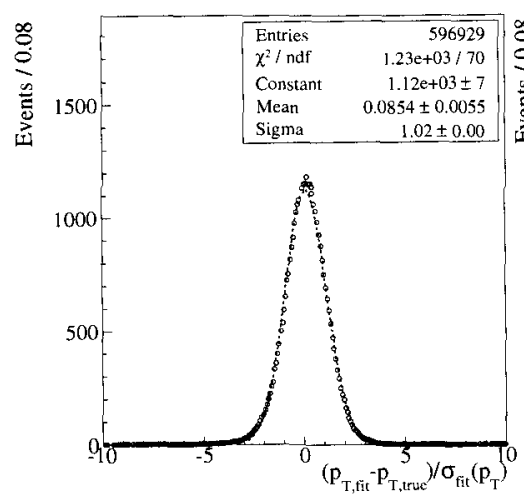

(a)

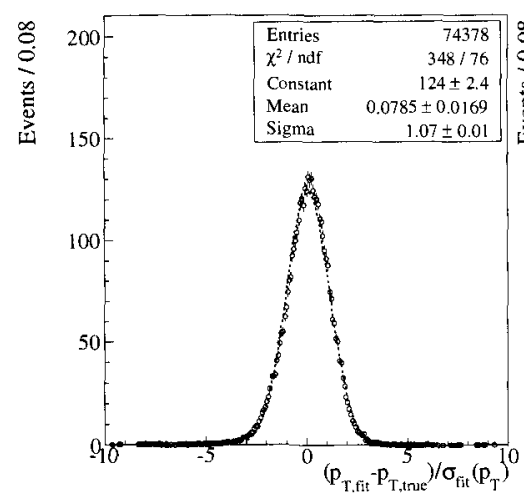

(c)

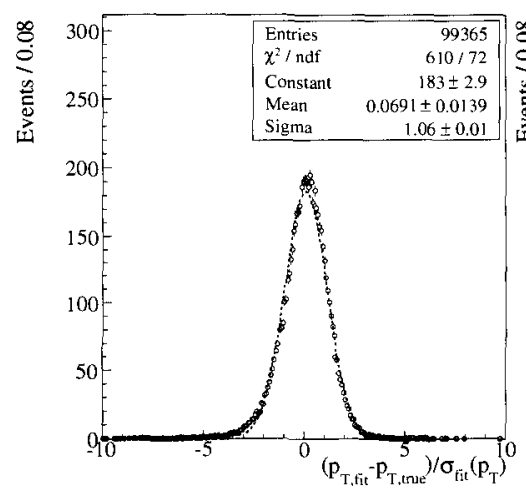

(e)

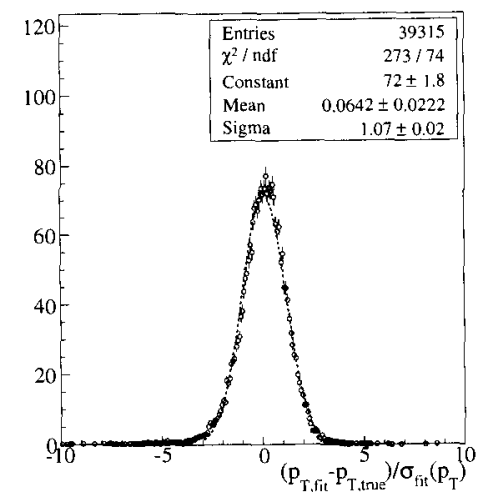

(b)

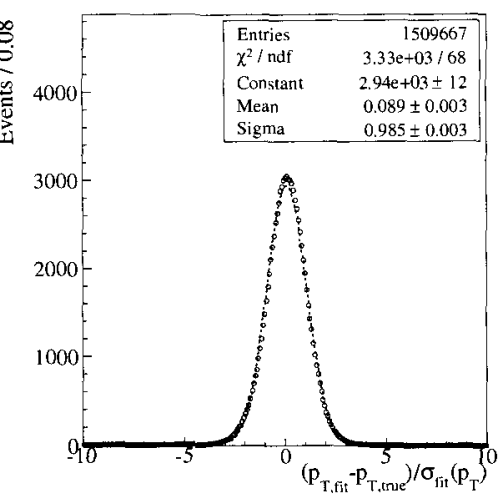

(d)

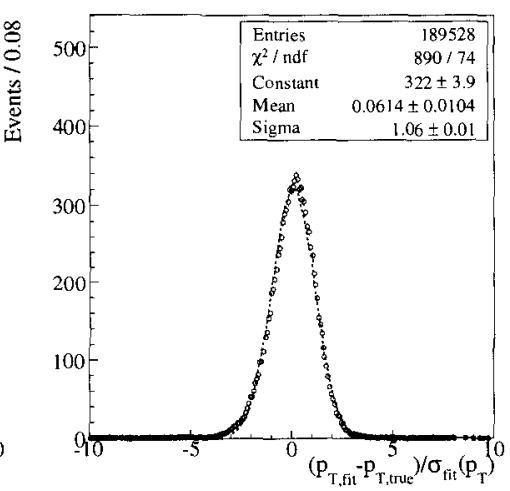

(f)

Figure 5.26: Fit pull distributions for central (a, d) and forward (b, e) muons with SMT hits, and muons without SMT hits (c, f). $\gamma^{\star} / Z$ Monte Carlo with muon resolutions 'smeared' to match pre-shutdown (a-c) and post-shutdown (d-f) data. 


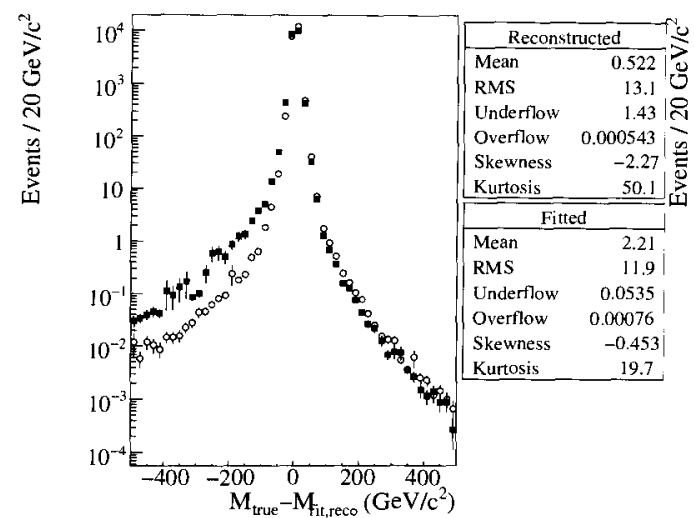

(a)

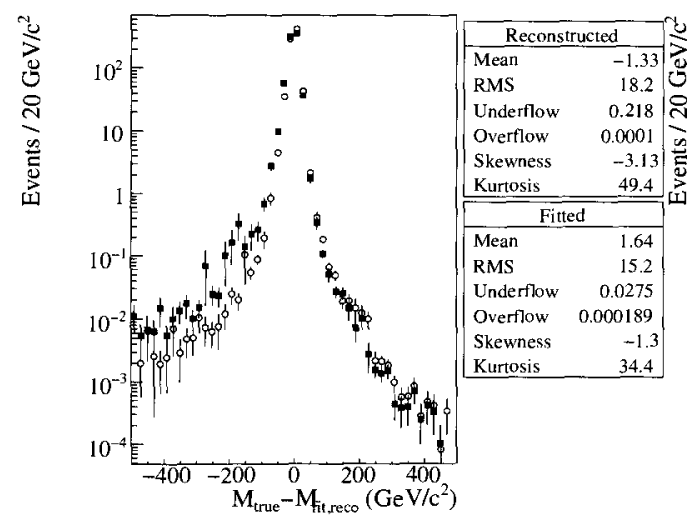

(c)

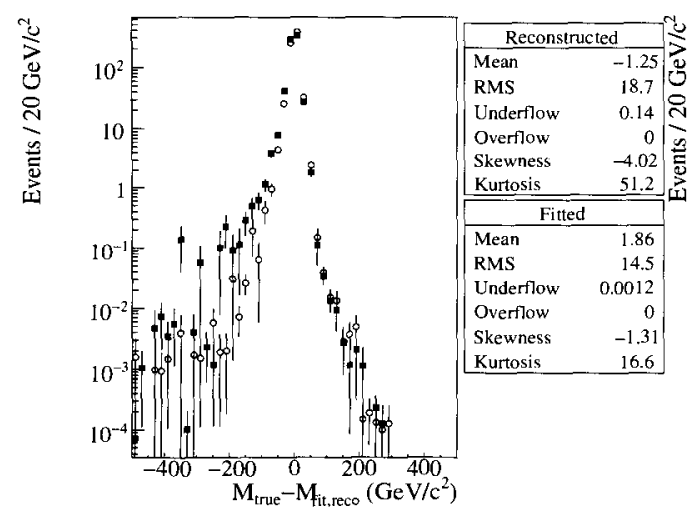

(e)

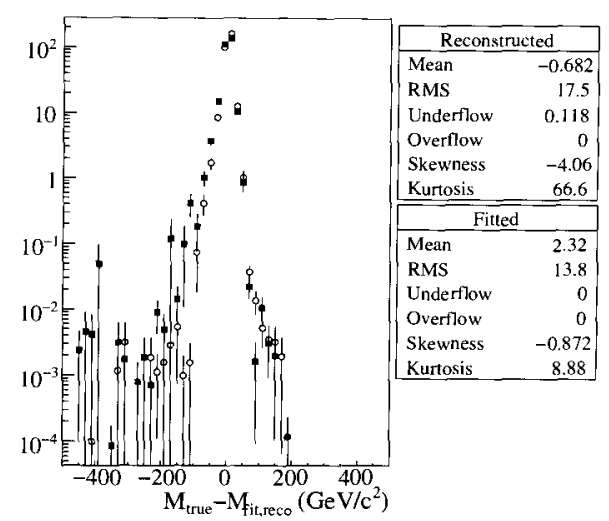

(b)

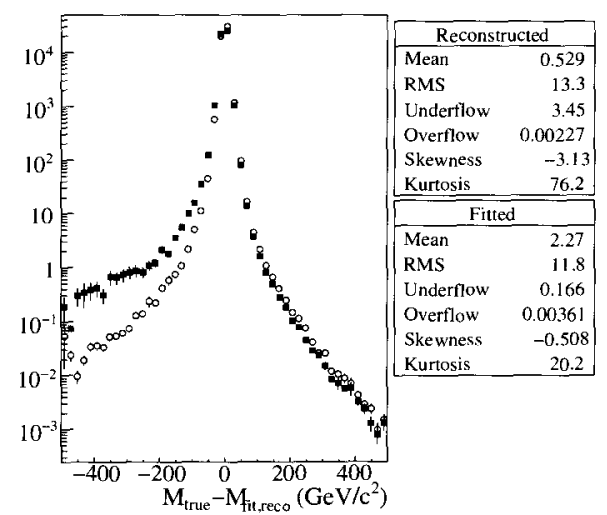

(d)

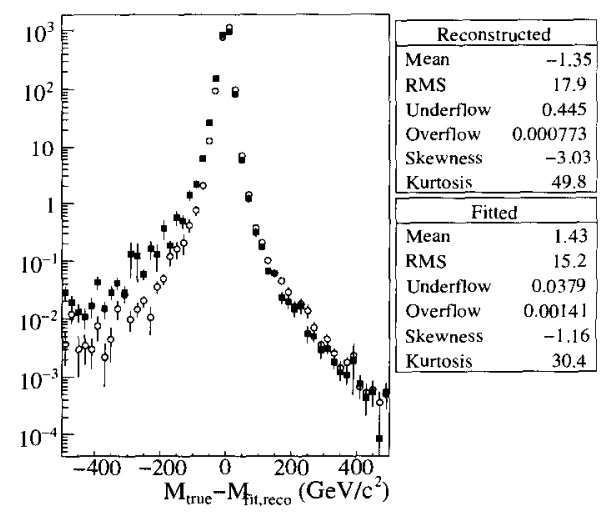

(f)

Figure 5.27: Difference of the generated vector boson mass with the reconstructed (closed squares) and with the fitted (open circles) dimuon mass respectively, for events with at least one selected central (a,d), or else forward (b, e) muon with SMT hits, or with both muons without SMT hits (c, f). $\gamma^{\star} / Z$ Monte Carlo with muon resolutions 'smeared' to match pre-shutdown (a-c) and post-shutdown (d-f) data. Errors are due to Monte Carlo statistics only. 


\section{Chapter 6}

\section{Search for new heavy gauge bosons}

Having obtained estimates for the Standard Model (SM) background and the effective luminosity, the final step in the analysis essentially consists of counting the number of events in data and comparing this with theory. This is called a 'counting experiment'; the quantity of interest is the total cross-section and the relevant statistic is the number of observed events.

A range of models of new physics is investigated. To calculate the acceptances for the $Z^{\prime}$ models, the Standard Model-like $Z^{\prime}$ Monte Carlo samples described in section 4.1.2 were used; for the Randall-Sundrum model, the samples described in 4.1.2 are used.

A final selection cut on the invariant dimuon mass is made, and chosen such that it maximizes the sensitivity to the presence of a signal.

Since no significant excess over the expected Standard Model background is observed, a Bayesian $95 \%$ credibility limit on the signal cross section is calculated, taking into account systematic uncertainties on the effective luminosity and background. These limits are interpreted as a limit on the $Z^{\prime}$ mass. The expected limit and its variation are calculated as well. The limits for the Standard Model-like $Z^{\prime}$ cross section are also interpreted as limits for the $Z^{\prime}$ in Littlest Higgs scenarios, and in a model-independent way. The limits for the Randall-Sundrum model are interpreted as limits on $M_{G^{[1]}}$ and $k / M_{\text {Planck. }}$.

\subsection{Counting experiment}

The new physics models all involve the resonant production and decay of a new particle $Z^{\prime}$ with a cross-section $\sigma_{S}$, which depends in some model-dependent specific way on the mass $M_{Z^{\prime}}$ and other parameters. These models therefore predict an excess of events above the Standard Model background rate. We test these hypotheses by 
looking at the observable $N_{R}$ : the number of events counted in a chosen region $R$ of the event variable space, and compare it with the expected distributions for the number of SM events, $B_{R}$. R is specified by the (pre)selection cuts described in the previous chapters, plus one more selection cut on the dimuon invariant mass, which is chosen independently for each generated signal Monte Carlo sample.

Specifically, the hypothesis $H_{\sigma_{S}}$ being tested is that $N_{R}$ is distributed according to a Poisson distribution as

$$
p\left(N_{R} \mid H_{\sigma_{S}}\right)=e^{-B_{R}-S_{R}} \frac{\left(B_{R}+S_{R}\right)^{N_{R}}}{N_{R} !}
$$

Here, $B_{R}$ is the expected (most probable) number of background (i.e., Standard Model) events in $R, S_{R}$ is the number of signal events in $R$.

The number of signal events is related to the signal cross section as

$$
S_{R}=\mathcal{L}_{\text {eff }}(R) \cdot \sigma_{S}
$$

The signal cross section $\sigma_{S}$ depends on the resonance mass $M_{Z^{\prime}}$ and other parameters. For $\sigma_{S} \rightarrow 0$ we recover the Standard Model hypothesis $H_{0}$, which is the null hypothesis against which we test,

$$
p\left(N_{R} \mid H_{0}\right)=e^{-B_{R}} \frac{B_{R}^{N_{R}}}{N_{R} !}
$$

The quantity $\mathcal{L}_{\text {eff }}(R)$ is the effective luminosity for $R$, i.e.,

$$
\mathcal{L}_{\text {eff }}(R)=\mathcal{L} \cdot \epsilon(R)
$$

where $\mathcal{L}$ is the integrated luminosity for the data sample and $\epsilon(R)$ is the acceptance times trigger efficiency times selection efficiency.

As described in section 5.4.1, $\mathcal{L}_{\text {eff }}$ is calculated with respect to the $Z$ cross-section,

$$
\mathcal{L}_{\text {eff }}(R)=\frac{N_{Z \text { data }}}{\epsilon_{M C}(Z) \cdot \sigma_{Z \rightarrow \mu^{+} \mu^{-}}} \cdot \epsilon_{\mathrm{sel}}(R)
$$

where $\frac{N Z \text { data }}{\epsilon_{M C}(Z) \cdot \sigma_{Z \rightarrow \mu^{+} \mu^{-}}}$is the normalization to the theoretical $Z$ production cross section times branching ratio and $\epsilon_{\text {sel }}(R)$ is the (corrected) selection efficiency for the signal.

The selection efficiencies are calculated on the Monte Carlo simulations described in 4.1 .2 as

$$
\epsilon_{\mathrm{sel}}(R)=\frac{\sum_{\left\{x_{i} \in \mathrm{R}\right\}} w_{i} \cdot c_{i}}{\sum_{\left\{x_{i}\right\}} w_{i}}
$$

where $w_{i}$ are the Monte Carlo event weights and $c_{i}$ are the efficiency correction factors. The calculated efficiencies are listed in table 6.1 for the SM-like $Z^{\prime}$ model and table 6.2 for the RS model. 
A discovery of a signal of new physics can be claimed if the probability of observing at least $N_{R}$ events is sufficiently small given the null hypothesis

$$
\sum_{s=0}^{\infty} p\left(N_{R}+s \mid H_{0}\right)<\alpha
$$

where $p\left(N_{R} \mid H_{0}\right)$ is given by (6.2). $\alpha$ is the chosen significance level of the test; in the following, it is set to correspond to three standard deviations of a one-sided normal distribution, i.e., $\alpha \approx 1.3 \cdot 10^{-3}$.

A discovery will therefore only be claimed if more events are observed than the minimum number needed for discovery,

$$
N_{R}>N_{\min }\left(B_{R}, \alpha\right)
$$

If, on the other hand, fewer than $N_{\min }$ events are observed, we can attempt to put an upper limit on the number of signal events that could still have contributed to $N_{R}$. Because there is always a finite possibility that $N_{R}$ events are observed for any value of $\sigma_{S}$, one chooses a desired credibility level (CL) for the upper limit, such that $1-\mathrm{CL}$ is the plausibility that the true value of $\sigma_{S}$ lies in fact above the claimed upper limit. Here we make the conventional choice of $\mathrm{CL}=95 \%$ (or about $1.65 \sigma$ ).

Whether or not a signal can be excluded at a certain credibility level depends of course on the choice of the selection criteria $R$. The selection cuts described in the previous chapter were not optimized other than that they were chosen to be efficient (except the anti-cosmic cuts); heuristically, this makes sense because the number of signal events is expected to be small in comparison with the number of Drell-Yan background events for any value of these cuts, while other remaining backgrounds are negligible.

However, this is not true for the cut on the invariant mass of the selected muon pair $M_{\mu \mu}$. This cut,

$$
M_{\text {lower }}<M_{\mu \mu}<M_{\text {upper }}
$$

is therefore optimized to maximize the sensitivity to the presence of a signal over the Drell-Yan (and other remaining) background.

It is a priori not clear how sensitivity should be defined and what is then the optimal choice for $M_{\text {lower }}, M_{\text {upper }}$. Common approaches include: the choice that leads to the highest significance in the case of a discovery, the largest expected range of discovery for a given model, or the largest expected range for exclusion in case there is no signal. These are not in general identical choices, and can lead to counter-intuitive results in pathological cases.

Here, the definition of [86] is used instead, which avoids these complications. The argument from that paper is summarized and applied in the following. 


\subsubsection{Sensitivity Optimization}

Let $1-\beta_{\alpha}\left(R ; \sigma_{S}, M_{Z^{\prime}}, \ldots\right)$ be the power of the test (6.4). This is the probability of discovering the signal at a significance level $\alpha$ in the case that $H_{\sigma_{S}}$ is actually true, i.e., that a new gauge boson actually exists. The "sensitivity region" of an experiment is defined as the region in the space of signal parameters $\left(\sigma_{S}, M_{Z^{\prime}}, \ldots\right)$ for which holds that

$$
1-\beta_{\alpha}\left(R ; \sigma_{S}, M_{Z^{\prime}}, \ldots\right)>\mathrm{CL}
$$

where CL is the chosen confidence level (95\%). In words, the sensitivity region of an experiment is the region in parameter space for which the probability to observe a signal at a significance level $\alpha$ when that signal indeed exists is greater than or equal to the confidence level chosen for the exclusion limits.

That is, if the true values of the parameters are such that equation (6.5) holds, there is a probability of at least CL that a discovery will be made at a significance $\alpha$. If, on the other hand, no discovery is made, at least this region can be excluded at a credibility level equal to or greater than CL.

Thus equation 6.5 describes the values of the signal parameters for which the experiment is "sufficiently sensitive" to definitely give an answer, either as a discovery, or as an exclusion at a predefined CL.

The "optimal" sensitivity is then reached for the choice of $R$ (in this case, the choice of $M_{\text {lower }}$ and $M_{\text {upper }}$ ) which leads to the largest sensitivity region.

In all models under investigation the cross section $\sigma_{S}$ decreases monotonically with $M_{Z^{\prime}}$; also, the effective luminosity is only dependent on $M_{Z^{\prime}}$, and is calculated for a number of discrete points in $M_{Z^{\prime}}$. Therefore, the problem is reduced to finding for each mass point the choice of $M_{\text {lower }}$ and $M_{\text {upper }}$ which leads to largest sensitivity range in $\sigma$.

Since the power of a counting experiment increases monotonically with the number of signal events $S$, equation (6.5) is satisfied if

$$
S_{R}\left(\sigma_{S}, M_{Z^{\prime}}, \ldots\right)>S_{\min }\left(B_{R}, \alpha, \mathrm{CL}\right)
$$

for some number of events $S_{\min }$ which only depends on $B_{R}$ and the choice of $\alpha$ and CL. Both $S_{R}$ and $B_{R}$ depend on the choice of $\left(M_{\text {lower }}, M_{\text {upper }}\right)$, but since the signal effective luminosity is independent of $\sigma_{S}$, this can be simplified to

$$
\sigma_{S}\left(M_{Z^{\prime}, \ldots}\right)>S_{\min }\left(B_{R}, \alpha, \mathrm{CL}\right) / \mathcal{L}_{\text {eff }}(R) \equiv \sigma_{\min }
$$

The maximal sensitivity is then obtained for the choice of $M_{\text {lower }}$ and $M_{\text {upper }}$ which leads to the lowest value of $\sigma_{\min }$.

$S_{\min }$ can in principle be calculated from (6.1) or computed numerically, but this leads to unworkable complicated expressions. Instead, in [86] an expansion orders of $\alpha$ and $\mathrm{CL}$ was fit to the exact answer. This leads to the following approximate expression for $S_{\min }$, 


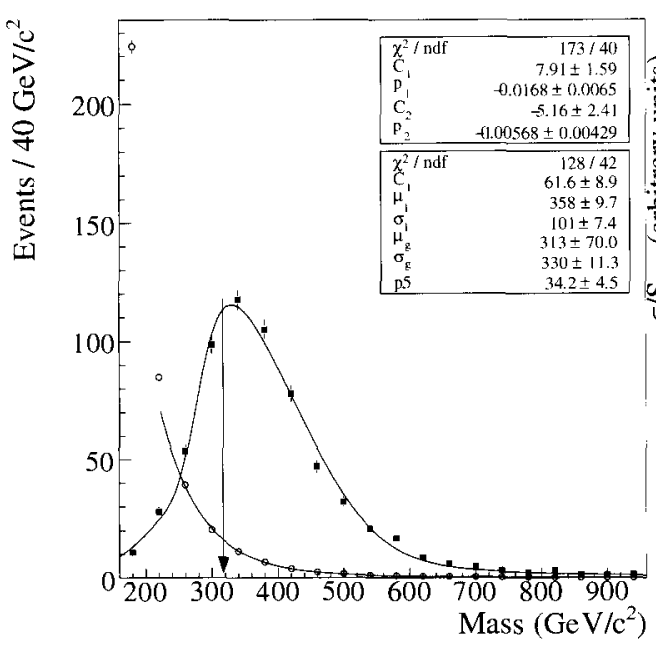

(a)

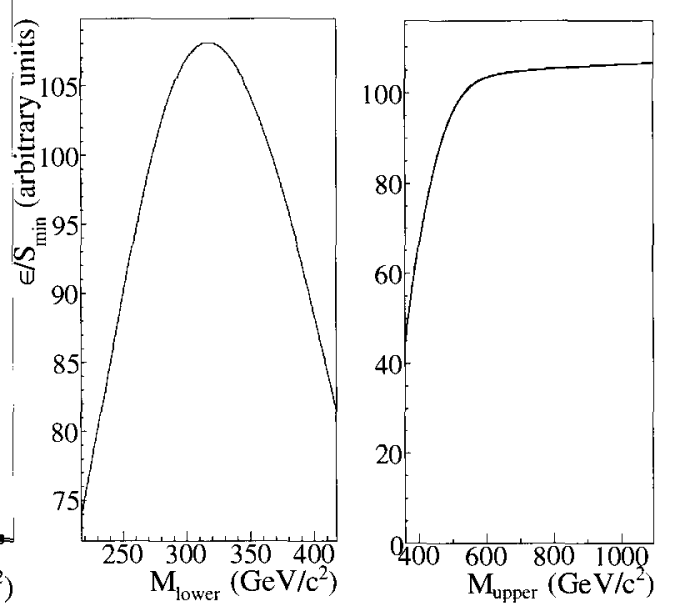

(b)

Figure 6.1: (a) Standard Model background (open circles) and Standard Model-like $Z^{\prime}$ with $M_{Z^{\prime}}=400 \mathrm{GeV} / c^{2}$ (squares), fitted with the functions (6.8) and (6.7) respectively; (b) $\epsilon / S_{\min }$ (equation (6.6)) as a function of $M_{\text {lower }}$ and $M_{\text {upper }}$ around the maximum with the other variable held fixed.

$$
S_{\min }=\frac{1}{8} a^{2}+\frac{9}{13} b^{2}+a \sqrt{B_{R}}+\frac{1}{2} b \sqrt{b^{2}+4 a \sqrt{B_{R}}+4 B_{R}}
$$

Here, $a$ and $b$ are the number of standard deviations corresponding to one-sided Gaussian tests for $\alpha(3 \sigma)$ and CL $(95 \% \sim 1.65 \sigma)$ respectively

The quantity that has to be maximized is then

$$
\frac{\epsilon_{\text {mass }}}{S_{\text {min }}}\left(M_{\text {lower }}, M_{\text {upper }}\right)
$$

where $\epsilon_{\text {mass }}$ is the efficiency of the cut on the invariant mass. The difference with the exact calculation is negligible.

Comparing the quantity (6.6) with a more usual quantity (a measure of the expected significance in the presence of a signal), $S / \sqrt{B}$, one can see that maximizing (6.6) will lead to a higher efficiency. The difference is larger for the case that the number of background events is small. This is in itself desirable because $S / \sqrt{B}$ diverges when $B \rightarrow 0$, so that maximizing it biases to low efficiency for small number of background events. For $B \rightarrow \infty$ the two expressions converge.

\section{Mass cut optimization}

To find the maximum of the expression (6.6), suitable functions are fitted to the signal and background distributions. The signal distributions were fitted with the following 


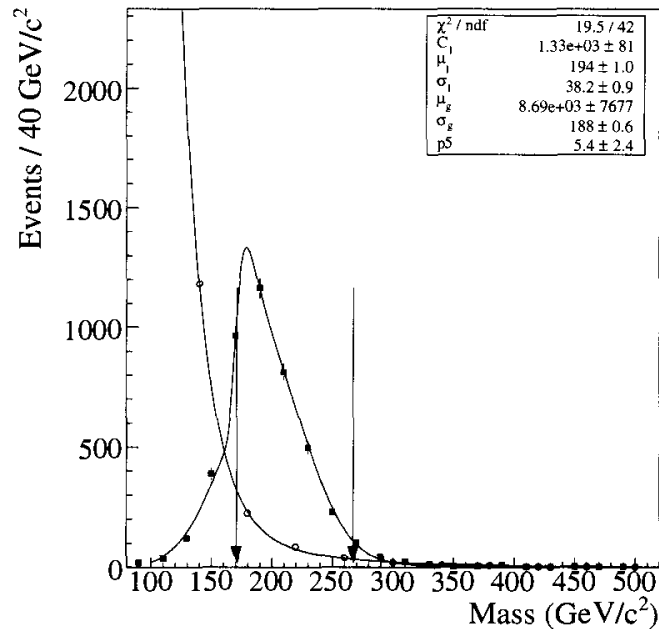

(a)

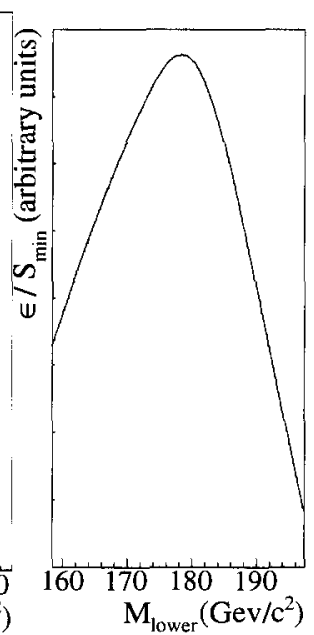

(b)

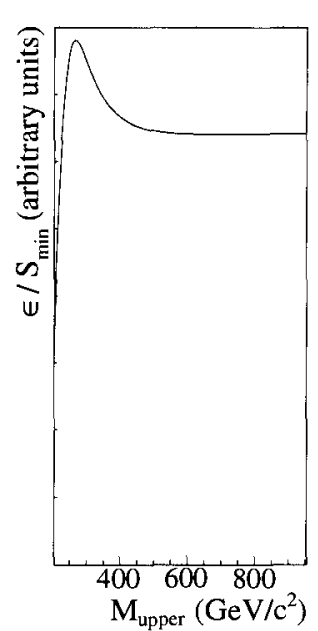

Figure 6.2: (a) Standard Model background (open circles) and the lowest mass graviton excitation in the Randall-Sundrum model with $M_{G^{[1]}}=200 \mathrm{GeV} / c^{2}$ (squares), fitted with the functions (6.8) and (6.7) respectively; (b) $\epsilon / S_{\min }$ (equation (6.6)) as a function of $M_{\text {lower }}$ and $M_{\text {upper }}$ around the maximum with the other variable held fixed.

ad-hoc expression,

$$
\frac{d S}{d \sqrt{s}}=\left(C_{1} \cdot L\left(\sqrt{s} ; \mu_{l}, \sigma_{l}\right)+C_{2} \cdot G\left(\sqrt{s} ; \mu_{g}, \sigma_{g}\right)\right)
$$

where $L$ is the Landau distribution with median $\mu_{l}$ and width $\sigma_{l}$, and $G$ is a Gaussian distribution with mean $\mu_{g}$ and width $\sigma_{g}$. The background with the sum of two exponentials,

$$
\frac{d B}{d \sqrt{s}}=\left(C_{1} e^{-p_{1} \sqrt{s}}+C_{2} e^{-p_{2} \sqrt{s}}\right)
$$

As an example, figures (6.1) show the result for a Standard Model-like $Z^{\prime}$ model with $M_{Z^{\prime}}=400 \mathrm{GeV} / c^{2}$ and figures (6.2) for the Randall-Sundrum model with $M_{G^{[1]}}=$ $200 \mathrm{GeV} / c^{2}$. Also shown are graphs of the quantity $\mathcal{S}$ around the maximum. The results are qualitatively similar for all mass points and the differences of the fitted optimal cut with the binned result are small.

For all models, the actual solution has $M_{\text {upper }} \rightarrow \infty$, the only exception being the $200 \mathrm{GeV} / \mathrm{c}^{2}$ Randall-Sundrum model, where the optimum lies at $M_{\text {upper }}=268 \mathrm{GeV} / c^{2}$.

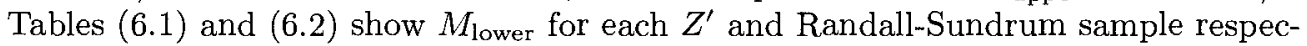
tively. 


\subsection{Bayesian credibility limits}

The $95 \%$ Bayesian upper credibility limit on the signal cross section is calculated for each mass point under consideration.

In the absence of a signal, the credibility of the true value of the signal cross-section $\sigma$ (in the following, the subscript $S$ is implicit) to lie in the range $(\sigma, \sigma+d \sigma)$, given the number of observed events $N$, is the posterior likelihood density $L(\sigma \mid N)$, with

$$
L(\sigma \mid N)=\frac{P(N \mid \sigma) \pi(\sigma)}{\pi(N)}
$$

where

$$
\pi(N)=\int P(N \mid \sigma) \pi(\sigma) d \sigma
$$

is a normalization factor, independent of $\sigma . \pi(\sigma)$ is called the 'prior density'. In the following, it is taken to be uniform in $\sigma$,

$$
\pi(\sigma)= \begin{cases}1 / \sigma_{\max } & , 0 \leq \sigma<\sigma_{\max } \\ 0 & , \text { otherwise }\end{cases}
$$

with $\sigma_{\max }$ large enough so that $P\left(N \mid \sigma_{\max }\right)$ is arbitrarily small for any value of $N$. This is not the least informative (or Jeffrey's) prior for p.d.f. (6.1), but it has the advantage that the coverage is equal to the classical ("frequentist") definition of coverage.

$P(N \mid \sigma)$ is given by the Poisson distribution $p\left(N \mid \sigma, \mathcal{L}_{\text {eff }}, B\right)$ of equation (6.1), convoluted with the p.d.f.'s for $\mathcal{L}_{\text {eff }}$ (called $\pi(l)$ ) and $B$ (called $\pi(b)$ );

$$
P(N \mid \sigma)=\iint p(N \mid \sigma, b, l) \pi(l) \pi(b) d b d l
$$

where $\pi(l)$ and $\pi(b)$ are are given by two-sided truncated Gaussians,

$$
\pi(l)=\frac{2}{\sqrt{2 \pi}} \frac{N}{\left(\sigma_{+}+\sigma_{-}\right)} \begin{cases}\exp \left(-\frac{1}{2}\left(\frac{\left(l-\mathcal{L}_{\text {eff }}\right)}{\sigma_{+}}\right)^{2}\right) & , l \geq \mathcal{L}_{\text {eff }} \\ \exp \left(-\frac{1}{2}\left(\frac{\left(l-\mathcal{L}_{\text {eff }}\right)}{\sigma_{-}}\right)^{2}\right) & , 0 \leq l<\mathcal{L}_{\text {eff }} \\ 0 & , l<0\end{cases}
$$

where $\sigma_{+}$and $\sigma_{-}$are the positive and negative systematic uncertainties on $\mathcal{L}_{\text {eff }}$, quoted below, and $N$ is a normalization constant. Similarly,

$$
\pi(b)=\frac{2}{\sqrt{2 \pi}} \frac{N}{\left(\sigma_{+}+\sigma_{-}\right)} \begin{cases}\exp \left(-\frac{1}{2}\left(\frac{(b-B)}{\sigma_{+}}\right)^{2}\right) & , b \geq B \\ \exp \left(-\frac{1}{2}\left(\frac{(b-B)}{\sigma_{-}}\right)^{2}\right) & , 0 \leq b<B \\ 0 & , b<0\end{cases}
$$


with $\sigma_{+}$and $\sigma_{-}$the systematic uncertainties on the expected background $B$.

The upper $95 \%$ credibility limit on the signal cross-section, $\sigma_{\text {upper }}$, is then found by solving numerically

$$
\int_{0}^{\sigma_{\text {upper }}} P(N \mid \sigma) d \sigma=0.95
$$

I.e., finding $\sigma_{\text {upper }}$ so that there is a plausibility of $95 \%$ that the true value of $\sigma$ lies inside the one-sided interval $\left[0, \sigma_{\text {upper }}\right]$.

\section{Expected Limits}

In addition, the expected limit is calculated; specifically, the mean upper limit on the signal cross-section one would expect to find in the absence of a signal,

$$
\left\langle\sigma_{\text {upper, } \exp }\right\rangle=\sum_{n} e^{-B} \frac{B^{n}}{n !} \sigma_{\text {upper }}\left(n, B, \mathcal{L}_{\text {eff }}\right)
$$

where $\sigma_{\text {upper }}\left(n, B, \mathcal{L}_{\text {eff }}\right)$ is the upper $95 \% \mathrm{CL}$ on the signal cross-section for $n$ observed events, and $n$ is distributed as in (6.1) with $\sigma \rightarrow 0$.

The (positive and negative) systematic variations on the average expected limit due to the systematic uncertainty on the expected number of observed events, $\Delta_{\text {(syst) }}^{ \pm}\left\langle\sigma_{\text {upper, exp }}\right\rangle$, are then calculated as

$$
\left\langle\sigma_{\text {upper, exp }}\right\rangle \mp \Delta_{\text {(syst) }}^{\mp}\left\langle\sigma_{\text {upper, } \exp }\right\rangle=\sum_{n} e^{-\left(B \pm \Delta^{ \pm} B\right)} \frac{\left(B \pm \Delta^{ \pm} B\right)^{n}}{n !} \sigma_{\text {upper }}\left(n, B, \mathcal{L}_{\text {eff }}\right)
$$

where $\Delta^{ \pm} B$ is the total positive/negative systematic uncertainty on the background, described below.

The variation around the average expected limit due to random fluctuations of the stochastic variable $n$, the number of observed events, is given by

$$
\Delta_{\text {(stat) }}^{2}\left(\sigma_{\text {upper, exp }}\right)=\sum_{n} e^{-B} \frac{B^{n}}{n !}\left(\sigma_{\text {upper }}\left(n, B, \mathcal{L}_{\text {eff }}\right)-\left\langle\sigma_{\text {upper, exp }}\right\rangle\right)^{2}
$$

\subsubsection{Systematic uncertainties on signal efficiency}

The systematic uncertainties on the muon identification, track reconstruction and isolation efficiencies were described in the previous chapter. Since the Monte-Carlo is normalized to the $Z$ peak, only the dimuon mass-dependent systematic effects are taken into account, i.e.,

$$
\Delta^{ \pm} \mathcal{L}_{\text {eff }}=\left|\mathcal{L}_{\text {eff }}-N^{ \pm} \epsilon^{ \pm}\right|
$$




\begin{tabular}{|c|c|c|c|c|c|c|c|c|}
\hline$M_{Z^{\prime}}$ & $M_{\text {lower }}$ & $\epsilon_{\operatorname{mass}}(\%)$ & $\epsilon_{\text {tot }}(\%)$ & muon ID & isolation & track & PDF & Total \\
\hline 400 & 317 & 71 & 31 & $\pm 0.5 \%$ & $\pm 0.1 \%$ & $\pm 0.1 \%$ & $\begin{array}{l}+0.3 \% \\
-0.2 \%\end{array}$ & $\begin{array}{l}+0.7 \% \\
-0.6 \%\end{array}$ \\
\hline 500 & 399 & 63 & 27 & $\pm 0.6 \%$ & $\pm 0.1 \%$ & $\pm 0.2 \%$ & $\begin{array}{l}+0.3 \% \\
-0.3 \%\end{array}$ & $\begin{array}{l}+0.7 \% \\
-0.7 \%\end{array}$ \\
\hline 600 & 481 & 57 & 25 & $\pm 0.6 \%$ & $\pm 0.1 \%$ & $\pm 0.2 \%$ & $\begin{array}{l}+0.4 \% \\
-0.6 \%\end{array}$ & $\begin{array}{l}+0.8 \% \\
-0.8 \%\end{array}$ \\
\hline 650 & 514 & 55 & 23 & $\pm 0.7 \%$ & $\pm 0.1 \%$ & $\pm 0.2 \%$ & $\begin{array}{l}+0.8 \% \\
-0.6 \%\end{array}$ & $\begin{array}{l}+1.1 \% \\
-0.9 \%\end{array}$ \\
\hline 700 & 566 & 51 & 22 & $\pm 0.7 \%$ & $\pm 0.1 \%$ & $\pm 0.2 \%$ & $\begin{array}{l}+0.7 \% \\
-1.0 \%\end{array}$ & $\begin{array}{l}+1.0 \% \\
-1.2 \%\end{array}$ \\
\hline 750 & 588 & 51 & 22 & $\pm 0.7 \%$ & $\pm 0.1 \%$ & $\pm 0.3 \%$ & $\begin{array}{l}+1.0 \% \\
-1.6 \%\end{array}$ & $\begin{array}{l}+1.2 \% \\
-1.7 \%\end{array}$ \\
\hline 800 & 618 & 48 & 20 & $\pm 0.5 \%$ & $\pm 0.1 \%$ & $\pm 0.2 \%$ & $\begin{array}{l}+1.3 \% \\
-2.0 \%\end{array}$ & $\begin{array}{l}+1.4 \% \\
-2.0 \%\end{array}$ \\
\hline 850 & 643 & 48 & 20 & $\pm 0.6 \%$ & $\pm 0.2 \%$ & $\pm 0.2 \%$ & $\begin{array}{l}+1.7 \% \\
-2.4 \%\end{array}$ & $\begin{array}{l}+1.8 \% \\
-2.5 \%\end{array}$ \\
\hline 900 & 669 & 45 & 18 & $\pm 0.6 \%$ & $\pm 0.1 \%$ & $\pm 0.2 \%$ & $\begin{array}{l}+2.4 \% \\
-3.3 \%\end{array}$ & $\begin{array}{l}+2.5 \% \\
-3.4 \%\end{array}$ \\
\hline 1000 & 718 & 37 & 13 & $\pm 0.7 \%$ & $\pm 0.1 \%$ & $\pm 0.3 \%$ & $\begin{array}{l}+4.9 \% \\
-6.3 \% \\
\end{array}$ & $\begin{array}{l}+4.9 \% \\
-6.4 \% \\
\end{array}$ \\
\hline
\end{tabular}

Table 6.1: For each mass point in the Standard Model-like $Z^{\prime}$ is shown the lower mass cut, the mass cut efficiency $\epsilon_{\text {mass }}$, the total signal selection efficiency $\epsilon_{\text {tot }}$ and the relative uncertainties on the total signal selection efficiency.

\begin{tabular}{|l|ll|l|lllll|}
\hline$M_{G^{[1]}}$ & $M_{\text {lower }}$ & $\epsilon_{\text {mass }}(\%)$ & $\epsilon_{\text {tot }}(\%)$ & muon ID & isolation & track & PDF & Total \\
\hline 200 & 178 & 67 & 29 & $\pm 0.6 \%$ & $\pm 0.1 \%$ & $\pm 0.1 \%$ & $+0.8 \%$ & $+1.0 \%$ \\
300 & 238 & 80 & 34 & $\pm 0.7 \%$ & $\pm 0.2 \%$ & $\pm 0.2 \%$ & $+0.3 \%$ & $-1.3 \%$ \\
400 & 321 & 70 & 29 & $\pm 0.8 \%$ & $\pm 0.1 \%$ & $\pm 0.1 \%$ & $+0.8 \%$ & $-2.0 \%$ \\
500 & 405 & 64 & 27 & $\pm 0.8 \%$ & $\pm 0.1 \%$ & $\pm 0.1 \%$ & $+1.5 \%$ & $-0.8 \%$ \\
600 & 498 & 57 & 23 & $\pm 0.7 \%$ & $\pm 0.1 \%$ & $\pm 0.2 \%$ & $-0.2 \%$ & $-0.7 \%$ \\
700 & 569 & 56 & 24 & $\pm 0.7 \%$ & $\pm 0.1 \%$ & $\pm 0.2 \%$ & $-0.8 \%$ & $-1.1 \%$ \\
800 & 642 & 55 & 23 & $\pm 0.7 \%$ & $\pm 0.1 \%$ & $\pm 0.1 \%$ & $+2.1 \%$ & $+2.2 \%$ \\
900 & 688 & 56 & 23 & $\pm 0.7 \%$ & $\pm 0.1 \%$ & $\pm 0.3 \%$ & $+0.6 \%$ & $-0.9 \%$ \\
1000 & 749 & 54 & 22 & $\pm 0.7 \%$ & $\pm 0.1 \%$ & $\pm 0.2 \%$ & $+0.8 \%$ & $+1.0 \%$ \\
\hline
\end{tabular}

Table 6.2: For each mass point in the Randall-Sundrum model is shown the lower mass cut, the mass cut efficiency $\epsilon_{\text {mass }}$, the total signal selection efficiency $\epsilon_{\text {tot }}$ and the relative uncertainties on the total signal selection efficiency. For $M_{G^{[1]}}=200 \mathrm{GeV} / \mathrm{c}^{2}$, the dimuon invariant mass is also required to be below $M_{\text {upper }}=268 \mathrm{GeV} / \mathrm{c}^{2}$. 


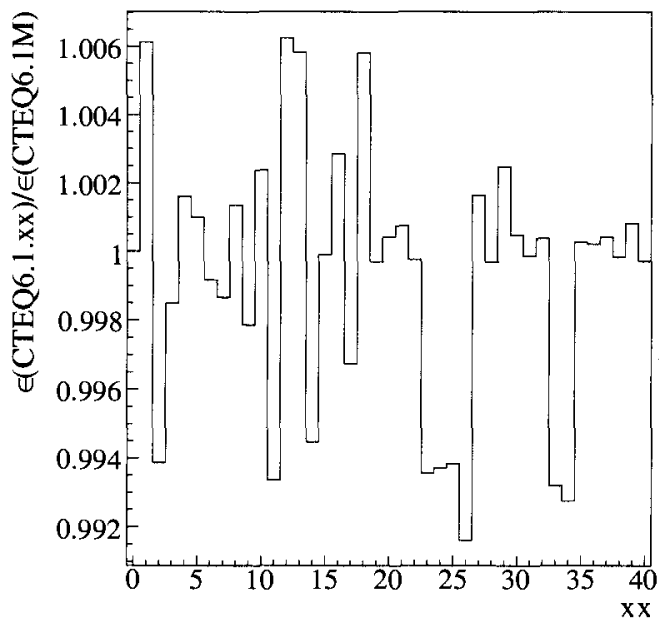

(a)

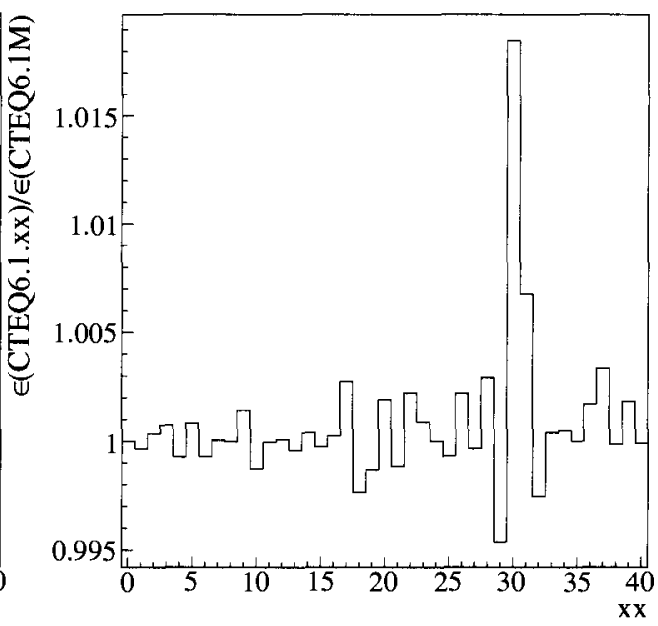

(b)

Figure 6.3: For each error set CTEQ6.1.xx is shown the ratio of the total selection efficiency to the central value (CTEQ6.1M, $\mathrm{xx}=0$ ), for an $800 \mathrm{GeV} / c^{2} Z^{\prime}$ (a) and an $800 \mathrm{GeV} / c^{2}$ RS graviton (b).

where $N^{ \pm}$is the Monte Carlo normalization (see section 5.4.1) calculated for the Standard Model Monte-Carlo with the positive or negative systematic, and $\epsilon^{ \pm}$is the signal efficiency with the same systematic.

\section{PDF uncertainty}

The uncertainty on the signal efficiency due to the PDF uncertainties was calculated following the prescription of [72]. The CTEQ collaboration provides a collection of $2 \times 20$ error sets with the CTEQ6.1M PDF. These represent a basis of "barely tolerable" fits, which have a $\chi^{2}=\chi_{0}^{2}+T^{2}$, where $\chi_{0}^{2}$ is of the optimal fit (i.e., the CTEQ6.1M PDF) and $T$ is the "tolerance" which is chosen to be $T \sim 10$. For more details see [72].

Each of the 40 CTEQ6.1 error sets CTEQ6.1.xx $(\mathrm{xx}=1 \ldots 40)$ is summed over, counting the squared, respectively positive and negative, differences with the central value estimate of the efficiency.

$$
\Delta_{\mathrm{PDF}}^{+} \epsilon_{\mathrm{sel}}=\sqrt{\sum_{\mathrm{xx}=1}^{40}\left(\epsilon_{\mathrm{sel}}(\mathrm{CTEQ} 6.1 . \mathrm{xx})-\epsilon_{\mathrm{sel}}(\mathrm{CTEQ} 6.1 \mathrm{M})\right)^{2}}
$$

summing over the PDF error sets for which $\epsilon_{\text {sel }}($ CTEQ6.1.xx $)>\epsilon_{\text {sel }}($ CTEQ6.1M), and summing over the other sets for $\Delta_{\mathrm{PDF}}^{-} \epsilon_{\mathrm{sel}}$. Here, the central estimate $\epsilon_{\text {sel }}$ (CTEQ6.1M) is given by equation 6.3 , and the displaced values $\epsilon_{\text {sel }}$ (CTEQ6.1.xx) 


\begin{tabular}{|ccc|}
\hline$M_{Z^{\prime}}$ & $p_{T}$ resolution & PDF \\
\hline 400 & $-3.0 \%+5.1 \%$ & $-3.4 \%+2.4 \%$ \\
500 & $-4.7 \%+5.5 \%$ & $-3.8 \%+2.6 \%$ \\
600 & $-7.1 \%+8.1 \%$ & $-4.0 \%+2.8 \%$ \\
650 & $-6.0 \%+9.1 \%$ & $-4.1 \%+2.8 \%$ \\
700 & $-9.3 \%+9.2 \%$ & $-4.2 \%+2.9 \%$ \\
750 & $-8.2 \%+13.1 \%$ & $-4.4 \%+3.0 \%$ \\
800 & $-10.1 \%+11.3 \%$ & $-4.4 \%+3.0 \%$ \\
850 & $-11.4 \%+12.2 \%$ & $-4.4 \%+3.0 \%$ \\
900 & $-10.5 \%+9.0 \%$ & $-4.5 \%+3.0 \%$ \\
1000 & $-9.5 \%+10.8 \%$ & $-4.5 \%+3.0 \%$ \\
\hline
\end{tabular}

\begin{tabular}{|c|c|c|}
\hline$M_{G^{\{1\}}}$ & $p_{T}$ resolution & PDF \\
\hline 200 & $-1.7 \%+2.6 \%$ & $-1.7 \%+1.2 \%$ \\
\hline 300 & $-1.4 \%+3.0 \%$ & $-2.7 \%+1.9 \%$ \\
\hline 400 & $-3.1 \%+4.7 \%$ & $-3.4 \%+2.4 \%$ \\
\hline 500 & $-4.9 \%+6.3 \%$ & $-3.8 \%+2.6 \%$ \\
\hline 600 & $-6.1 \%+8.8 \%$ & $-4.1 \%+2.8 \%$ \\
\hline 700 & $-9.9 \%+8.8 \%$ & $-4.2 \%+2.9 \%$ \\
\hline 800 & $-11.4 \%+12.2 \%$ & $-4.4 \%+3.0 \%$ \\
\hline 900 & $-10.0 \%+8.3 \%$ & $-4.5 \%+3.0 \%$ \\
\hline 1000 & $-10.0 \%+9.9 \%$ & $-4.6 \%+3.0 \%$ \\
\hline
\end{tabular}

Table 6.3: Uncertainties on number of background events for each mass point, (a) Standard Model-like $Z^{\prime}$ and (b) Randall-Sundrum model.

are calculated with scaled Monte Carlo weights $w_{i}^{\prime}=w_{i} \cdot \frac{W(\mathrm{CTEQ6} \cdot 1 . \mathrm{xx})}{W(\mathrm{CTEQ6} \cdot 1 . \mathrm{M})}$ where the $W$ 's are the appropriate PDF weights.

Tables 6.1 and 6.2 list these systematic uncertainties for the $Z^{\prime}$ and RS models respectively. As an example, figures 6.3 show for each error set the ratio of the computed efficiency to the central value, for an $800 \mathrm{GeV} / c^{2}$ SM-like $Z^{\prime}$ and an $800 \mathrm{GeV} / c^{2} G^{[1]}$. The first is not dominated by one error set in particular, but for the RS model the PDF error is dominated by CTEQ6.1.30 (the error set CTEQ6.1.30 is driven by the gluon density, which is not well constrained at high $x$ ). This picture is qualitatively similar for $Z^{\prime}$ s and $G^{[1]}$ s of other masses.

\subsubsection{Systematic uncertainty on background}

The following systematic uncertainties on the number of background events are taken into account:

- The uncertainty on the normalization,

$$
\Delta^{-} n=4.0 \%, \Delta^{+} n=4.3 \%
$$

taken from the $Z$ peak fit error, the error on the theoretical $Z$ peak cross section and the difference between the normalization with and without using the kinematic fit (see 5.4.1).

- The uncertainty on the normalized invariant mass distribution due to the statistical plus systematic uncertainties on the muon resolution (figure 5.4(b)); see 5.3.1. 
- The uncertainty on the normalized Drell-Yan cross section due to the PDF uncertainty (see figure 4.3).

All systematic uncertainties are taken to be uncorrelated. If instead the uncertainty on the normalized Drell-Yan cross-section due to the PDF uncertainty is taken to be fully (positively) correlated with the error on the signal efficiency due to the PDF uncertainties, the resulting cross-section limits are $\lesssim 1.5 \%$ better than the found result (depending on the mass point); the difference on the found mass limit on the Standard Model-like $Z^{\prime}$ is negligible.

The results are summarized in tables 6.3 .

\subsubsection{Limits on Standard Model-like $Z^{\prime}$ cross section}

\begin{tabular}{|ccccccc|}
\hline$M_{Z^{\prime}}$ & $M_{\text {lower }}$ & $N_{\text {obs }}$ & \multicolumn{1}{l}{$N_{\text {bkg }}$} & $\mathcal{L}_{\text {eff }}\left(\mathrm{fb}^{-1}\right)$ & $\sigma_{\text {upper }}(\mathrm{fb})$ & $\sigma_{\text {upper }}(\exp ).(\mathrm{fb})$ \\
\hline 400 & 317 & 36 & $31.5_{-6.5 \%}^{+7.5 \%}$ & $30.8_{-0.6 \%}^{+0.7 \%}$ & 56 & $45_{-15}^{+16}$ \\
500 & 399 & 12 & $12.7_{-7.6 \%}^{+7.9 \%}$ & $26.7_{-0.7 \%}^{+0.7 \%}$ & 32 & $35 \pm 12$ \\
600 & 481 & 5 & $5.9_{-9.4 \%}^{+9.9 \%}$ & $24.9_{-0.8 \%}^{+0.8 \%}$ & 24 & $28 \pm 9$ \\
650 & 514 & 3 & $4.5_{-8.6 \%}^{+10.7 \%}$ & $23.4_{-0.9 \%}^{+1.1 \%}$ & 21 & $27 \pm 9$ \\
700 & 566 & 1 & $3.1_{-11.2 \%}^{+10.8 \%}$ & $21.7_{-1.2 \%}^{+1.0 \%}$ & 17 & $26 \pm 9$ \\
750 & 588 & 1 & $2.5_{-10.4 \%}^{+13.5 \%}$ & $22.0_{-1.7 \%}^{+1.2 \%}$ & 17 & $24 \pm 8$ \\
800 & 618 & 1 & $2.1_{-12.0 \%}^{+12.7 \%}$ & $20.2_{-2.0 \%}^{+1.4 \%}$ & 19 & $25 \pm 8$ \\
850 & 643 & 1 & $1.8_{-13.1 \%}^{+13.5 \%}$ & $19.8_{-2.5 \%}^{+2.8 \%}$ & 20 & $24 \pm 8$ \\
900 & 669 & 1 & $1.5_{-12.3 \%}^{+10.7 \%}$ & $17.6_{-3.4 \%}^{+2.5 \%}$ & 23 & $26 \pm 8$ \\
1000 & 718 & 1 & $1.1_{-11.5 \%}^{+12.3 \%}$ & $13.5_{-6.4 \%}^{+4.9 \%}$ & 31 & $32 \pm 10$ \\
\hline
\end{tabular}

Table 6.4: Summary of results for Standard Model-like $Z^{\prime}$.

The result of the calculations described before are shown in table 6.4 and figure 6.4. Shown is the computed upper $95 \%$ credibility limit on the cross-section as a function of the $Z^{\prime}$ mass. In the same figure the cross-section times branching ratio of a Standard Model-like $Z^{\prime}$ was plotted. The lower $95 \%$ credibility limit on the SM-like $Z^{\prime}$ mass is $838_{-8}^{+5} \mathrm{GeV} / c^{2}$, where the error is due to the error on the signal cross-section coming from the PDF uncertainty relative to the $Z$ peak cross section [78] (see figure 4.3). ${ }^{1}$

Also shown is the cross section of a $Z^{\prime}$ in the littlest Higgs scenario (with the same acceptances as a SM-like $Z^{\prime}$ ) for $\cot \theta=1$. The mass limit for this model is $M_{Z_{H}}>$ $863 \mathrm{GeV} / c^{2}$.

\footnotetext{
${ }^{1}$ The effect of the variation of the cross section limit with the $Z^{\prime}$ mass was neglected in the calculation of this error, because for $M_{Z^{\prime}}$ between $830-843 \mathrm{GeV} / c^{2}$ the cross section limit happens to be nearly constant.
} 


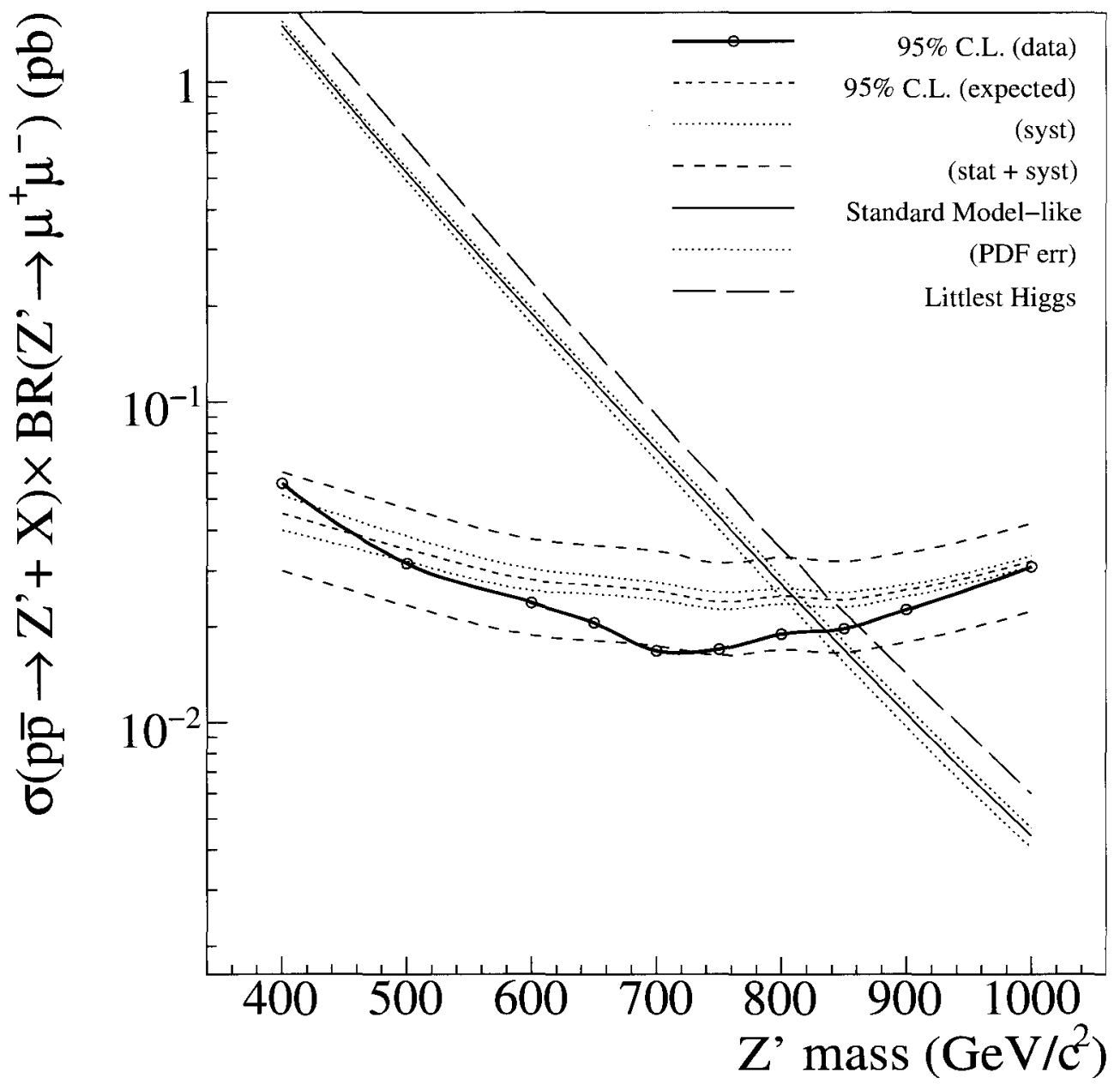

Figure 6.4: 95\% credibility limit on Standard Model-like $Z^{\prime}$ cross-section as a function of the $Z^{\prime}$ mass, and the average expected limit, the systematic uncertainty on the average expected limit and the systematic + statistical uncertainty. Also shown is the Standard Model-like NNLO $Z^{\prime}$ cross section times branching ratio, the error due to the PDF uncertainty and the (LO) Littlest Higgs $Z^{\prime}$ cross section times branching ratio for $\cot \theta=1$. 


\subsubsection{Limits for generic $Z^{\prime}$ models from extended gauge groups}

For a generic $Z^{\prime}$ model with an extra $U^{\prime}(1)$ extension to the Standard Model gauge group (see 2.3.3 for details), the production cross-section times branching ratio can be expressed as $[28]$

$$
\sigma\left(p \bar{p} \rightarrow Z^{\prime} X \rightarrow \mu^{+} \mu^{-} X\right)=\frac{\pi}{48 s}\left[c_{u} w_{u}\left(s, M_{Z^{\prime}}^{2}\right)+c_{d} w_{d}\left(s, M_{Z^{\prime}}^{2}\right)\right]
$$

where $w_{u, d}$ are the parts which do not depend on any $Z^{\prime}$ coupling, and were calculated for each $M_{Z^{\prime}}$ point under consideration using the CTEQ6.1M structure functions ${ }^{2}$.

The parameters $c_{u}$ and $c_{d}$ contain all dependence on the $Z^{\prime}$ couplings to fermions. Therefore, assuming the signal acceptance is independent of $c_{u, d}$, an upper limit on the SM-like $Z^{\prime}$ cross-section as a function of $M_{Z^{\prime}}$ corresponds to a limit in the $\left(c_{u}, c_{d}\right)$ plane, which can then be interpreted as a limit for any generic $Z^{\prime}$ model. The dependence of the signal acceptance on $c_{u, d}$ was investigated in [28] and found to be small.

Figure 6.5 shows the result. Also indicated are the regions in the $\left(c_{u}, c_{d}\right)$ plane corresponding to specific classes of models described in 2.3.3; the limit on the masses of the $Z_{\psi}, Z_{\eta}$ and $Z_{\chi}$ (see section 2.3 .3 ) were $665 \mathrm{GeV} / c^{2}, 654 \mathrm{GeV} / c^{2}$ and $790 \mathrm{GeV} / c^{2}$ respectively.

\subsubsection{Limits on Randall-Sundrum model}

\begin{tabular}{|ccccccc|}
\hline$M_{G^{[1]}}$ & $M_{\text {lower }}$ & $N_{\text {obs }}$ & \multicolumn{1}{l}{$N_{\text {bkg }}$} & $\mathcal{L}_{\text {eff }}\left(\mathrm{fb}^{-1}\right)$ & $\sigma_{\text {upper }}(\mathrm{fb})$ & $\sigma_{\text {upper }}(\exp ).(\mathrm{fb})$ \\
\hline 200 & 178 & 236 & $222.4_{-5.3 \%}^{+5.8 \%}$ & $29.3_{-1.3 \%}^{+1.0 \%}$ & 167 & $135_{-44}^{+49}$ \\
300 & 238 & 112 & $94.8_{-5.6 \%}^{+6.1 \%}$ & $33.6_{-1.0 \%}^{+0.8 \%}$ & 115 & $72_{-24}^{+25}$ \\
400 & 321 & 33 & $30.0_{-6.5 \%}^{+7.3 \%}$ & $29.1_{-0.8 \%}^{+1.0 \%}$ & 54 & $47 \pm 16$ \\
500 & 405 & 12 & $11.7_{-7.7 \%}^{+8.5 \%}$ & $26.6_{-1.2 \%}^{+1.7 \%}$ & 34 & $34_{-11}^{+12}$ \\
600 & 498 & 4 & $5.2_{-8.5 \%}^{+10.5 \%}$ & $23.3_{-0.7 \%}^{+0.8 \%}$ & 23 & $28_{-9}^{+10}$ \\
700 & 569 & 1 & $3.0_{-11.7 \%}^{+10.5 \%}$ & $23.8_{-1.1 \%}^{+1.6 \%}$ & 15 & $23 \pm 8$ \\
800 & 642 & 1 & $1.8_{-13.1 \%}^{+13.5 \%}$ & $22.6_{-0.9 \%}^{+2.2 \%}$ & 17 & $21 \pm 7$ \\
900 & 688 & 1 & $1.3_{-11.9 \%}^{+10.2 \%}$ & $23.3_{-1.7 \%}^{+1.0 \%}$ & 17 & $19 \pm 6$ \\
1000 & 749 & 1 & $0.9_{-11.9 \%}^{+11.5 \%}$ & $22.2_{-2.0 \%}^{+2.2 \%}$ & 19 & $19 \pm 6$ \\
\hline
\end{tabular}

Table 6.5: Summary of results for the Randall-Sundrum model.

The same calculations were done for the Randall-Sundrum model and are summarized in table 6.5. Figure 6.6 shows the $95 \%$ credibility limit on the presence of the lightest massive Randall-Sundrum graviton as a function of $M_{G^{[1]}}$, the expected limit and the

\footnotetext{
${ }^{2}$ Code kindly provided by M. Carena [28].
} 


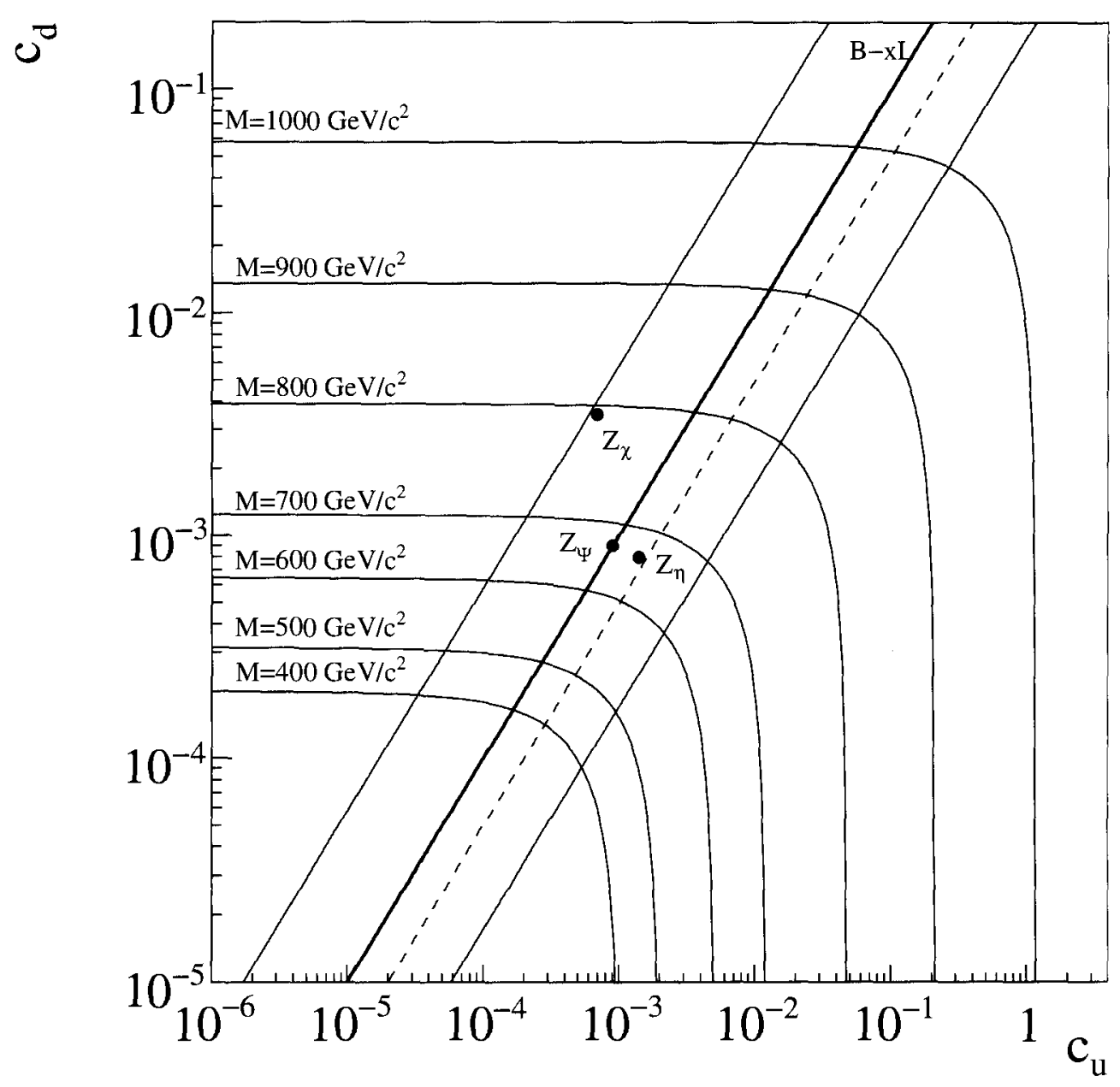

Figure 6.5: Excluded regions in $\left(c_{u}, c_{d}\right)$ plane; the area above the curved lines for fixed $M_{Z^{\prime}}$ is excluded. Also indicated are the regions of the $\left(c_{u}, c_{d}\right)$ plane allowed for certain model types; the thick solid line at $c_{u}=c_{d}$ covers the $B-x L$ models, the area between the thin solid lines covers the $q+x u$ models, and the area above the dashed line covers the $10+x \overline{5}$ models and the entire plane covers the $d-x u$ models (see section 2.3.3 for an explanation). Also indicated are the specific $E_{6}$ inspired models $Z_{\psi}, Z_{\eta}$ and $Z_{\chi}$. 


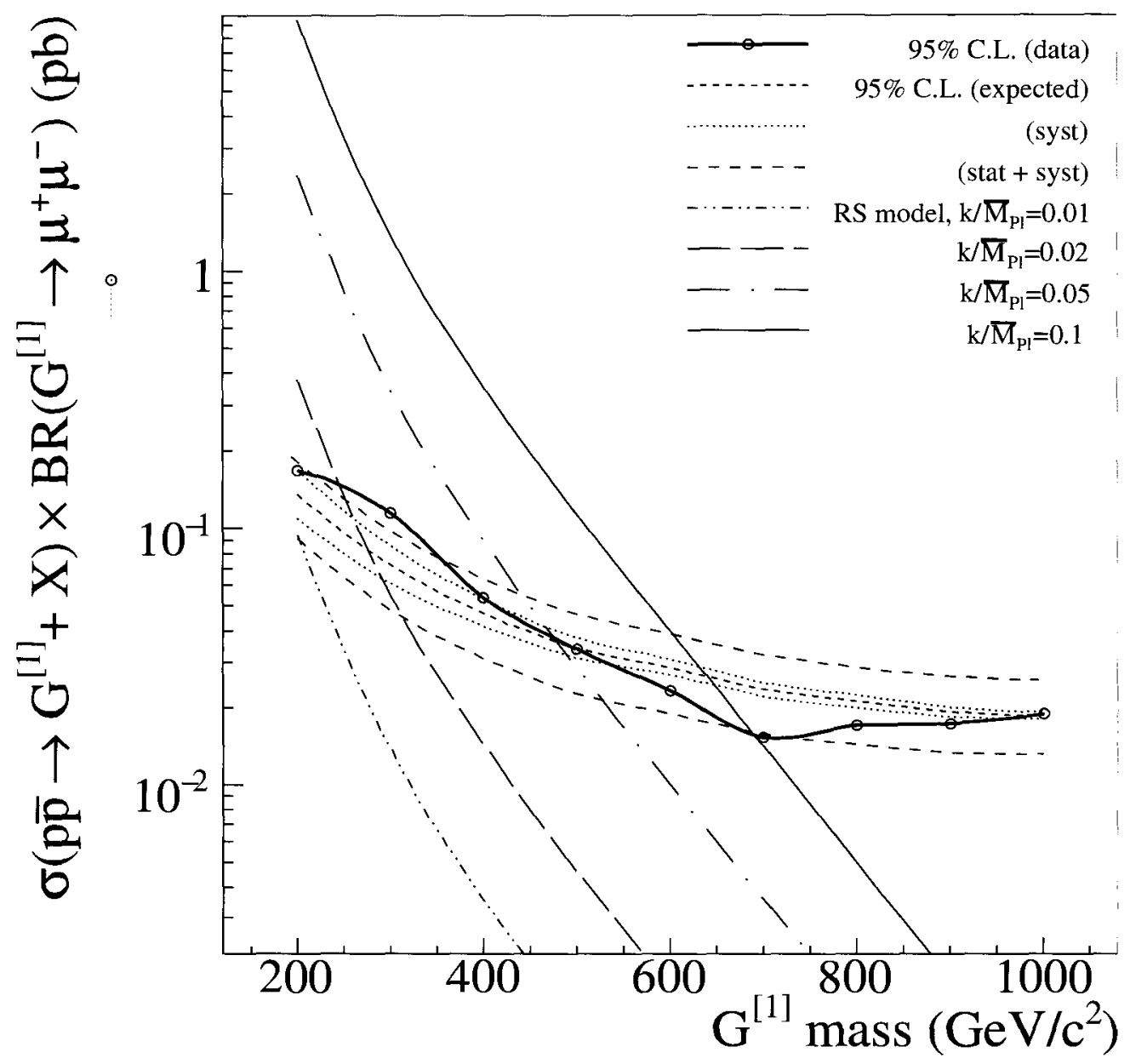

Figure 6.6: 95\% credibility limit on Randall-Sundrum production cross section times branching ratio as a function of the $G^{[1]}$ mass, and average expected limit. Also shown is the cross section in the RS model for different values of the parameter $k / \bar{M}_{\mathrm{Pl}}$. 
RS cross section for several values of $k / \bar{M}_{\mathrm{Pl}}$. Here, the variation of the width of the $\mathrm{RS}$ resonance was neglected; this is a good approximation because the reconstructed width is (by far) dominated by the mass resolution for $k / \bar{M}_{\mathrm{Pl}}$ between 0.01 and 0.1 . The difference of the limit for $M_{\left.G^{[1]}\right]}=300 \mathrm{GeV} / c^{2}$ with the expected limit is $1.7 \sigma$; this can be compared to the 'excess' of data events over the expected Standard Model background for a reconstructed dimuon mass of around $280 \mathrm{GeV} / c^{2}$, as can be seen in figure 5.24 .

For $k / \bar{M}_{\mathrm{Pl}}=0.1$ the limit is at $M_{G[1]}>693 \mathrm{GeV} / c^{2}$.

Figure 6.7 shows the observed and expected limits in the $\left(M_{G^{[1]}}, \frac{k}{M_{\mathrm{Pl}}}\right)$ plane. 


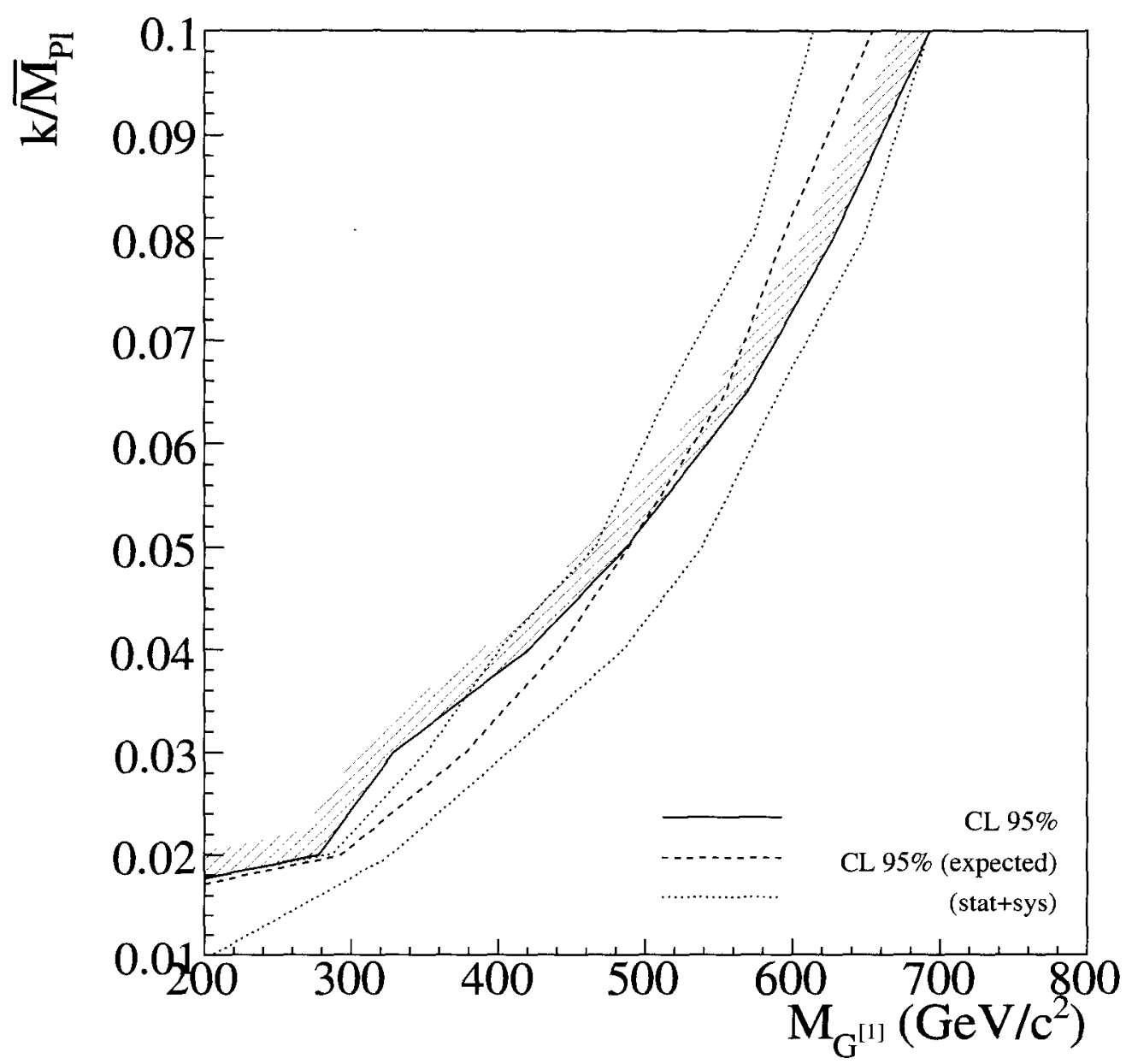

Figure 6.7: Observed and expected $95 \%$ credibility limit on $\left(M_{G^{[1]}}, \frac{k}{M_{\mathrm{Pl}}}\right)$. The area above the curve is excluded. 


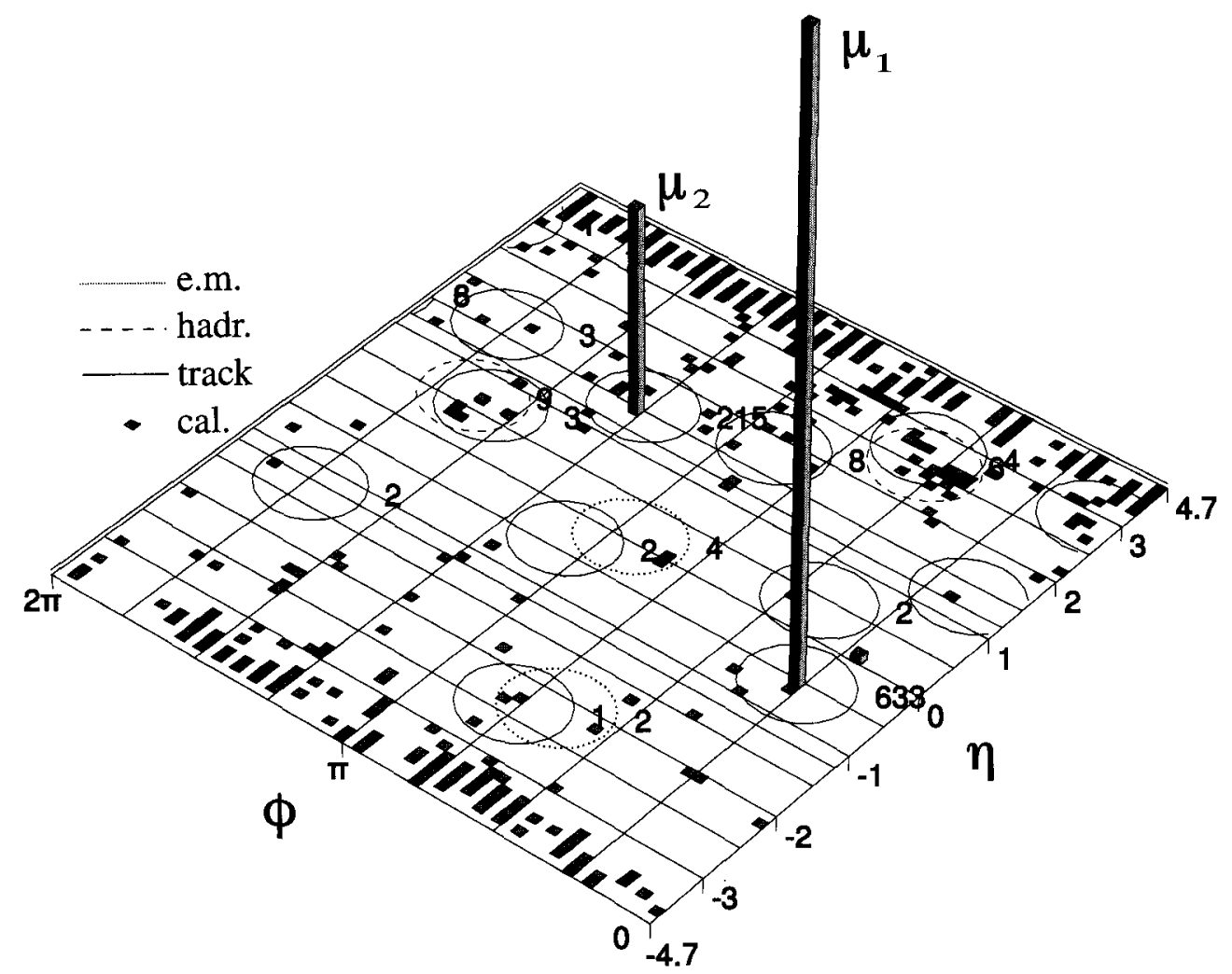

Figure 6.8: Lego plot showing the angle and transverse energies of reconstructed objects in the event with the largest dimuon invariant mass.

\subsection{Highest mass event}

Here we look in some detail at the event (run 213675, event 67615820) that had the highest reconstructed dimuon mass after the kinematic fit. Figure 6.8 schematically shows the configuration of the reconstructed objects and their transverse energies. Figure 6.9 shows a frontal view of the $D \varnothing$ detector, showing reconstructed tracks extrapolated to the muon system and calorimeter objects.

As events in hadron colliders go, this event is relatively clean. Although a large number of low energy tracks were present, only three tracks had a $p_{T}>10 \mathrm{GeV} / c$, the (by far) most energetic of which were matched to muon objects. One muon object $\left(\mu_{1}\right)$ was reconstructed from a large number of hits in all three layers, while the central track of the second muon object $\left(\mu_{2}\right)$ was matched only with forward scintillator hits, also in all three layers. $\mu_{2}$ thus satisfied the "loose" selection criteria that were used in this analysis, but not tighter requirements due to a number of missing hits in the muon system. Looking at figure 6.9 we see that the extrapolated central track and the matched calorimeter object point in the direction of the crack between two octants, 


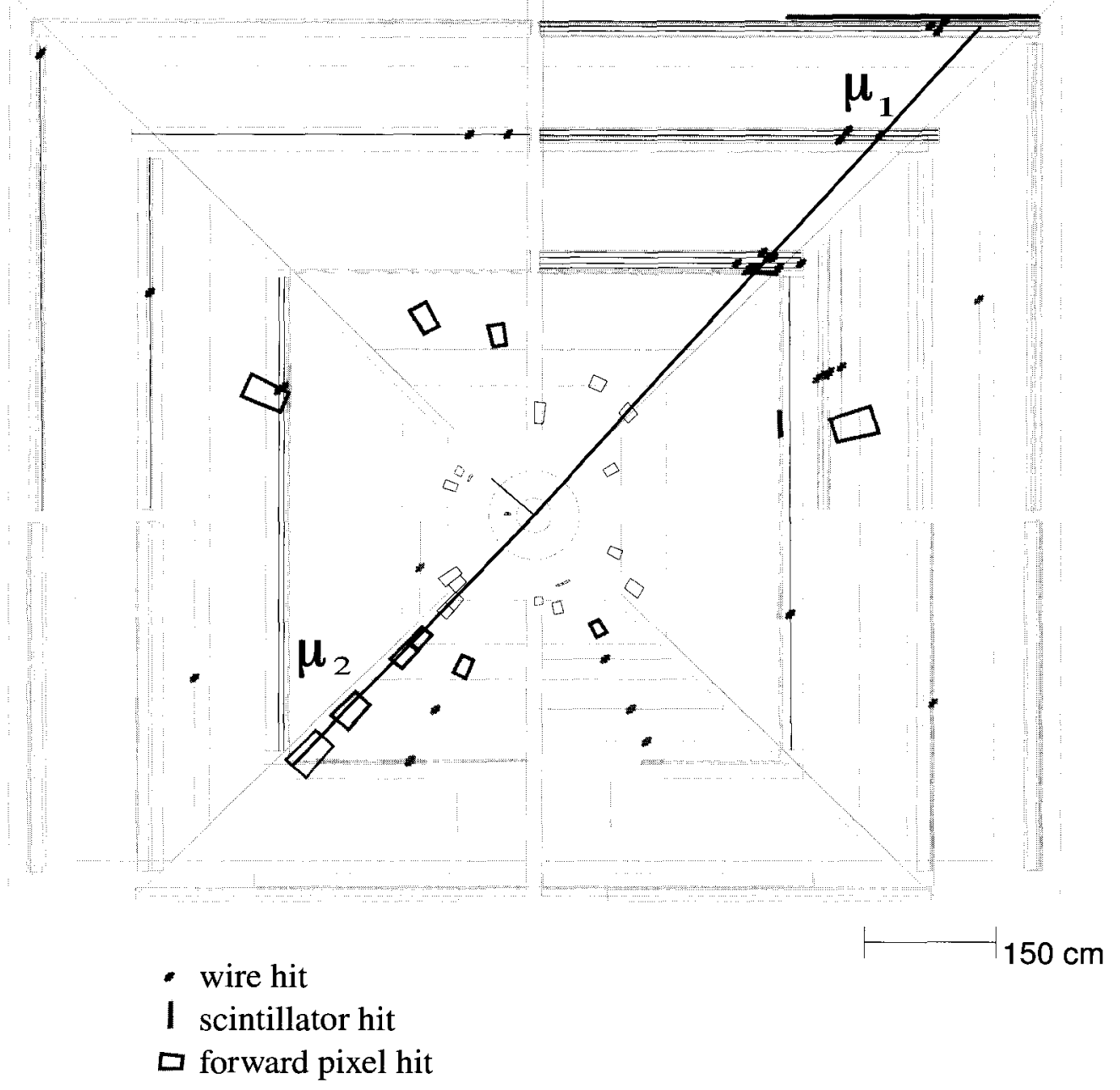

Figure 6.9: $\mathrm{X}-\mathrm{Y}$ view of the $\mathrm{D} \varnothing$ detector. For a detailed description, see the text. 
which may explain why the MDTs are missing some hits for this muon.

The muon objects were matched with extremely straight central tracks which were very nearly back-to-back $\left(\Delta \phi_{1,2}=3.12\right)$ in the transverse plane, exactly what one would expect from a high mass dimuon event. However, the track of $\mu_{1}$ had a reconstructed transverse momentum of $p_{T, 1}^{(\text {reco) }}=628 \mathrm{GeV} / c$ while the track of $\mu_{2}$ was reconstructed with only $p_{T, 2}^{(\text {reco) }}=209 \mathrm{GeV} / c$, even though no other high-energy objects were reconstructed. The resulting reconstructed dimuon invariant mass was $1140 \mathrm{GeV} / c^{2}$. Note that at $p_{T}^{(\text {reco) }}=628 \mathrm{GeV} / c$ the predicted (inverse) track resolution for a track with SMT hits is $\gtrsim 100 \%$ (see figure 5.25 ), so that an error like this is not unexpected at these energies. The missing calorimeter $E_{T}$ (omitting the muons) was only $8 \mathrm{GeV}$. Consequently, after the kinematic fit the fitted track $p_{T}$ 's were $p_{T, 1}^{\text {(fit) }}=312 \mathrm{GeV} / c$ and $p_{T, 2}^{(\text {fit })}=306 \mathrm{GeV} / c$ respectively, with a fitted $p_{T}(Z)=10 \mathrm{GeV} / c$ and a resulting dimuon invariant mass of $975 \mathrm{GeV} / c^{2}$. 


\section{Chapter 7}

\section{Conclusion \& Outlook}

We have used $1 \mathrm{fb}^{-1}$ of high- $p_{T}$ dimuon data taken by the D $\varnothing$ detector to look for a new heavy mass resonance. Since no significant excess over the Standard Model background was found, upper limits on the production cross-section times the branching ratio to muons were calculated for several models of new physics.

We set a lower $95 \%$ credibility limit on the mass of a hypothetical Standard Model-like $Z^{\prime}$ decaying to muons of $838_{-8}^{+5} \mathrm{GeV} / c^{2}$. These cross section limits were interpreted as limits on the mass and couplings for generic models with an extra gauge boson; the limits on the mass of the $Z_{\psi}, Z_{\eta}$ and $Z_{\chi}$ bosons that arise in various $E_{6}$ grand unified theories were constrained to be $665 \mathrm{GeV} / c^{2}, 654 \mathrm{GeV} / c^{2}$ and $790 \mathrm{GeV} / c^{2}$ respectively

We also set a limit for a spin-2 resonance predicted by the Randall-Sundrum model. The mass of the lightest massive graviton in the Randall-Sundrum model was constrained to be $M_{G^{[1]}}>693 \mathrm{GeV} / c^{2}$ for $k / \bar{M}_{\mathrm{Pl}}=0.1$ at a $95 \% \mathrm{CL}$.

These are the most stringent limits on $\sigma\left(p \bar{p} \rightarrow Z^{\prime}+X\right) \times \operatorname{BR}\left(Z^{\prime} \rightarrow \mu^{+} \mu^{-}\right)$and $\sigma\left(p \bar{p} \rightarrow G^{[1]}+X\right) \times \mathrm{BR}\left(G^{[1]} \rightarrow \mu^{+} \mu^{-}\right)$from direct searches to date.

As can be inferred from figures 6.4 and 6.6 , the results are limited by statistics. Assuming the systematic uncertainties are unchanged ${ }^{1}$, the (mean) expected limit on the mass of a hypothetical SM-like $Z^{\prime}$ (assuming no new physics) would be $907 \mathrm{GeV} / c^{2}$ with $4 \mathrm{fb}^{-1}$ of data, which is about the amount of data on tape by the time of writing, or $955 \mathrm{GeV} / c^{2}$ with $8 \mathrm{fb}^{-1}$ of data, which the Tevatron is expected to have delivered by the end of 2010 .

After that time the limits set by the LHC experiments might be much better, due to the larger energy range and luminosity available; a Standard Model-like $Z^{\prime}$ with a mass up to $2.5 \mathrm{TeV} / c^{2}$ could be excluded with $1 \mathrm{fb}^{-1}$ of LHC data and one with a mass up to $5 \mathrm{TeV} / \mathrm{c}^{2}$ with about $200 \mathrm{fb}^{-1}$ of LHC data $[87,88]$.

Similar direct searches have been done in dielectron and dielectron plus diphoton data at $\mathrm{D} \varnothing$ and $\mathrm{CDF}[32,33,48]$ (see table 2.3). For models with generation-independent fermion couplings, these analyses resulted in more stringent limits on the presence of

\footnotetext{
${ }^{1}$ The largest experimental systematic uncertainty comes from the uncertainty on the track resolution, which would be reduced by at most $\sim 30 \%$ with increased statistics (see table 5.3 ).
} 
heavy resonances, or similar limits with less data, because of the higher acceptance and better mass resolution that can be achieved for these channels. In addition, the search for RS gravitons in the $e^{+} e^{-}+\gamma \gamma$ channel has the advantage of the double branching ratio of the (spin-2) gravitons to photons.

Apart from the accumulation of more data, the inclusion of additional channels, and the combination with $\mathrm{CDF}$ searches, one possible way of improving this analysis would be to use the dimuon decay angle in addition to the dimuon invariant mass as a discriminating variable; this would however introduce additional model dependence to the limits. 


\section{Appendix A}

\section{Results with EW corrections}

The Drell-Yan background processes are reweighted to match the cross-section with NLO electroweak corrections included, as described in section 4.2. This changes the total $Z$ cross-section and thus the normalization of the Monte Carlo. It also changes the shape of the Drell-Yan backgrounds.

The second effect turns out to be small. Although the Drell-Yan background is much reduced at the Monte Carlo truth-level, the effect on the reconstructed background in the signal regions is small because the main source is not high-mass Drell-Yan events, but rather $Z$ events with a misreconstructed mass, for which the overall weight does not change because of the normalization to the $Z$ peak.

The signal Monte Carlo shape would in principle also change. However, it is argued in [28] that the NLO electroweak corrections to the $Z^{\prime}$ cross section are substantially smaller than the correction to the Standard Model neutral current. Moreover, the change in the width of the reconstructed mass distribution is dominated by resolution effects and the efficiency has only a small dependence on the invariant mass. Therefore the overall effect is likely to be small and the selection efficiency is assumed to be unchanged.

While the optimal mass cuts do change slightly after the EW correction, the changes are all $\lesssim 5 \mathrm{GeV} / c^{2}$ and are neglected as well. Since the systematic uncertainty due to the muon track resolution uncertainty on the normalized reconstructed Drell-Yan background depends mainly on the value of the mass cut (again, because the background is mainly due to misreconstructed $Z$ events), the relative systematic uncertainties are also left unchanged.

Figure A.1 show the $Z$ peak before and after the kinematic fit, figure A.2 the complete mass spectrum.

Table A.1 summarizes the result and figure A shows the $95 \%$ credibility limit on the presence of a Standard Model-like $Z^{\prime}$. 


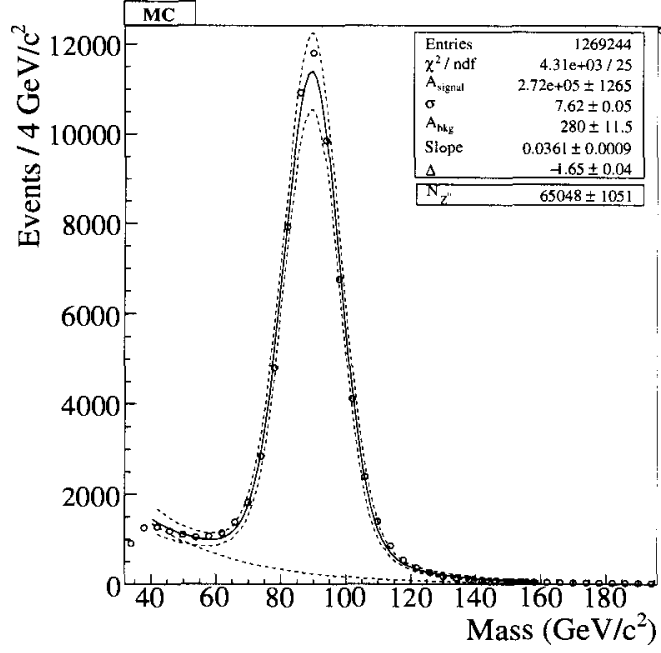

(a)

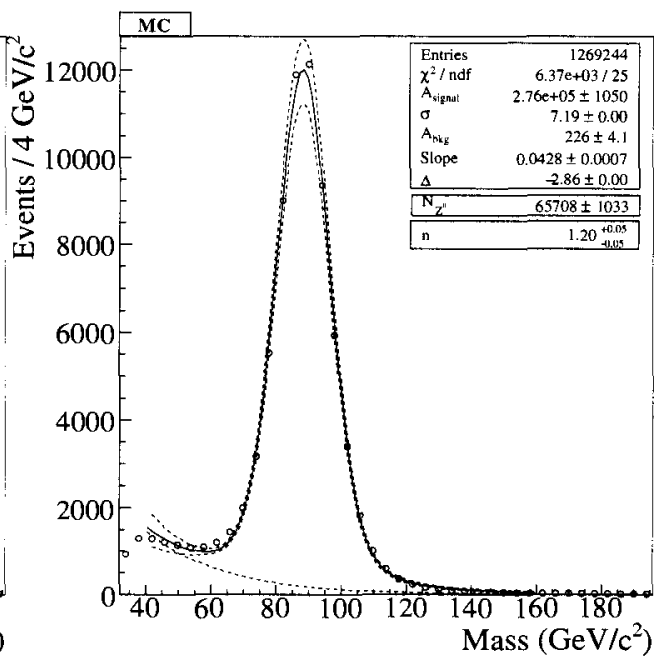

(b)

Figure A.1: Binned likelihood fit on the dimuon $Z$ resonance for events in final selection before (a) and after (b) the kinematic fit, Monte Carlo.

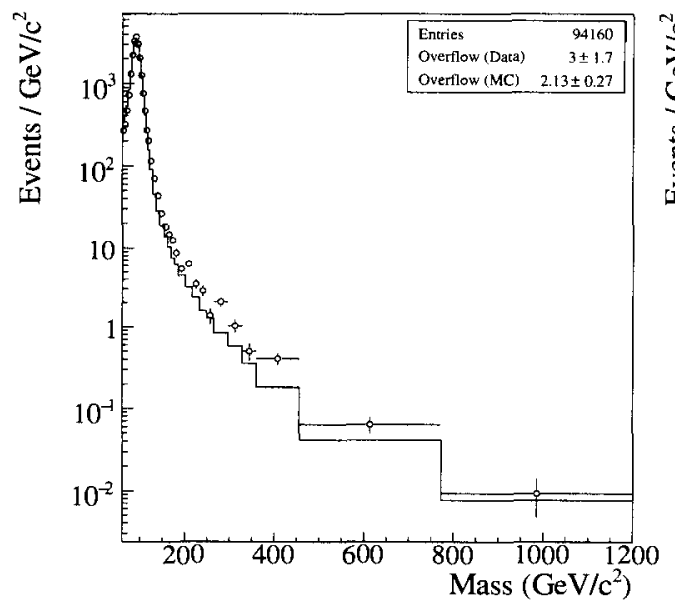

(a)

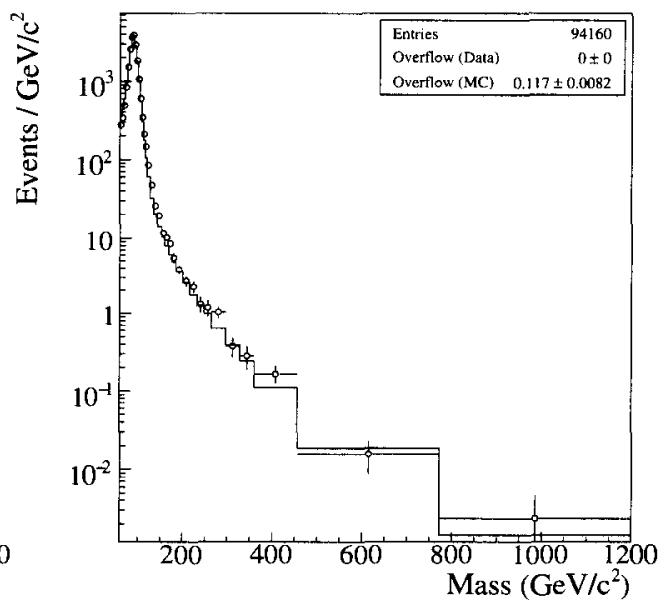

(b)

Figure A.2: Dimuon mass for events in final selection before (a) and after (b) the kinematic fit, data (open circles) and Monte Carlo (line). 


\begin{tabular}{|ccccccccc|}
\hline$M_{Z^{\prime}}$ & $M_{\text {lower }}$ & $N_{\text {obs }}$ & \multicolumn{1}{c}{$N_{\text {bkg }}$} & & \multicolumn{2}{c}{$\mathcal{L}_{\text {eff }}\left(\mathrm{fb}^{-1}\right)$} & CL $95 \%(\mathrm{fb})$ & (exp.) \\
\hline 400 & 317 & 36 & 29.1 & $-7 \%+8 \%$ & 31.1 & $-8.1 \%+6.0 \%$ & 64 & $45 \pm 15$ \\
500 & 399 & 12 & 11.7 & $-9 \%+9 \%$ & 27.1 & $-5.6 \%+6.6 \%$ & 34 & $34 \pm 11$ \\
600 & 481 & 5 & 5.5 & $-11 \%+11 \%$ & 25.1 & $-5.2 \%+6.3 \%$ & 25 & $27 \pm 9$ \\
650 & 514 & 3 & 4.1 & $-10 \%+12 \%$ & 23.6 & $-6.0 \%+7.1 \%$ & 21 & $26 \pm 9$ \\
700 & 566 & 1 & 2.8 & $-13 \%+12 \%$ & 21.9 & $-6.2 \%+7.4 \%$ & 17 & $25 \pm 8$ \\
750 & 588 & 1 & 2.3 & $-12 \%+16 \%$ & 22.1 & $-6.7 \%+8.0 \%$ & 17 & $23 \pm 8$ \\
800 & 618 & 1 & 1.9 & $-13 \%+14 \%$ & 20.5 & $-7.0 \%+7.9 \%$ & 19 & $24 \pm 8$ \\
850 & 643 & 1 & 1.6 & $-15 \%+15 \%$ & 20.4 & $-7.2 \%+8.2 \%$ & 19 & $23 \pm 8$ \\
900 & 669 & 1 & 1.3 & $-14 \%+12 \%$ & 18.3 & $-7.4 \%+8.4 \%$ & 22 & $25 \pm 8$ \\
1000 & 718 & 1 & 1.0 & $-13 \%+13 \%$ & 14.8 & $-4.7 \%+6.6 \%$ & 28 & $28 \pm 10$ \\
\hline
\end{tabular}

Table A.1: Summary of results for Standard Model-like $Z^{\prime}$.

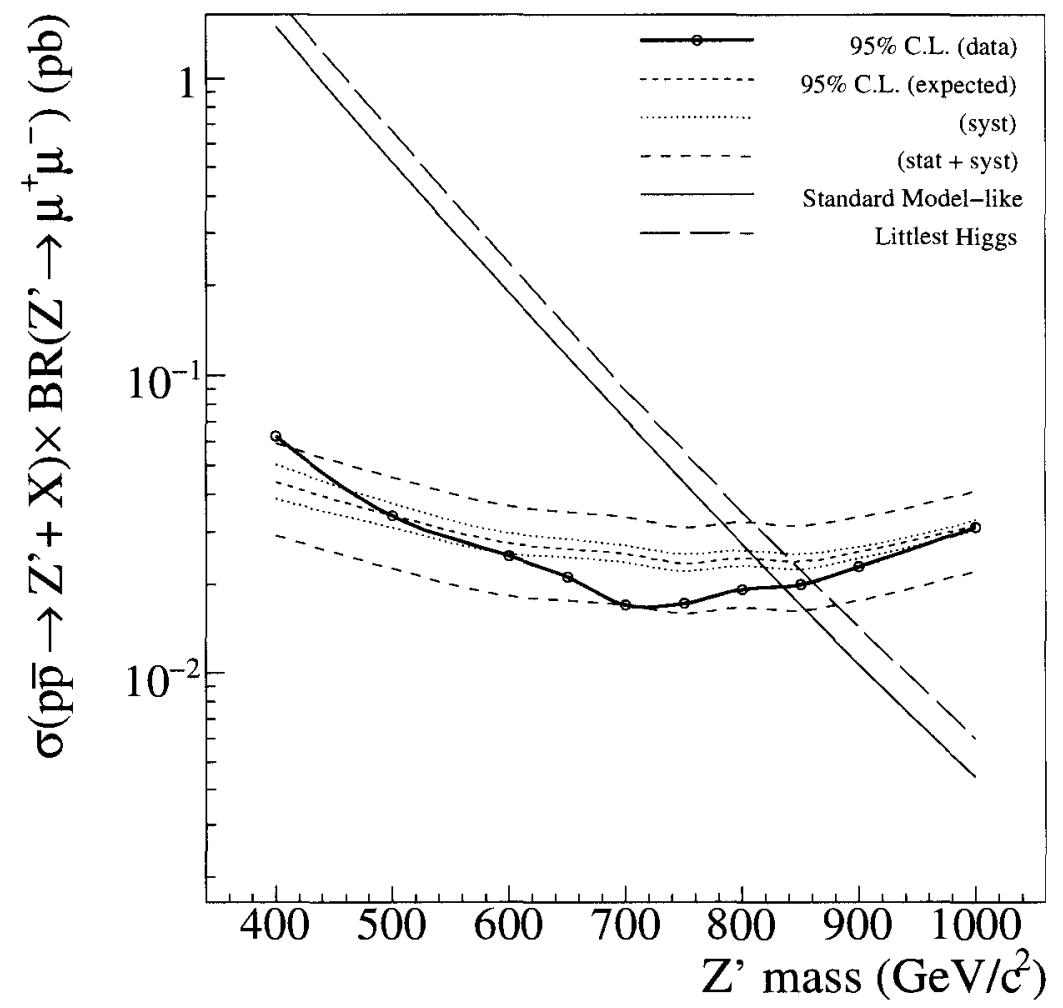

Figure A.3: 95\% credibility limit on Standard Model-like $Z^{\prime}$ cross-section as a function of the $Z^{\prime}$ mass, and the average expected limit, the systematic uncertainty on the average expected limit and the systematic + statistical uncertainty. Also shown is the Standard Model-like NNLO $Z^{\prime}$ cross section times branching ratio and the (LO) Littlest Higgs $Z^{\prime}$ cross section times branching ratio for $\cot \theta=1$. 


\section{Appendix B}

\section{Triggers}

Table B. 1 shows all triggers used, per trigger list period (see table 5.1 for the integrated luminosity per trigger list). This is simply the collection of all single- ("MU.*") and dimuon ("DMU.*" and "2MU.*") triggers used in Runlla. The nomenclature changed halfway v13. Triggers may typically require a number of hits in the muon system, hits in the central tracker at Level 1, a muon track and possibly a central track at Level 2. Some triggers require a track match at level 3. A few triggers in v14 require an isolated track at level 3 ("ITK"). Many of the single muon triggers are prescaled, especially at the higher luminosities with later trigger list versions. 


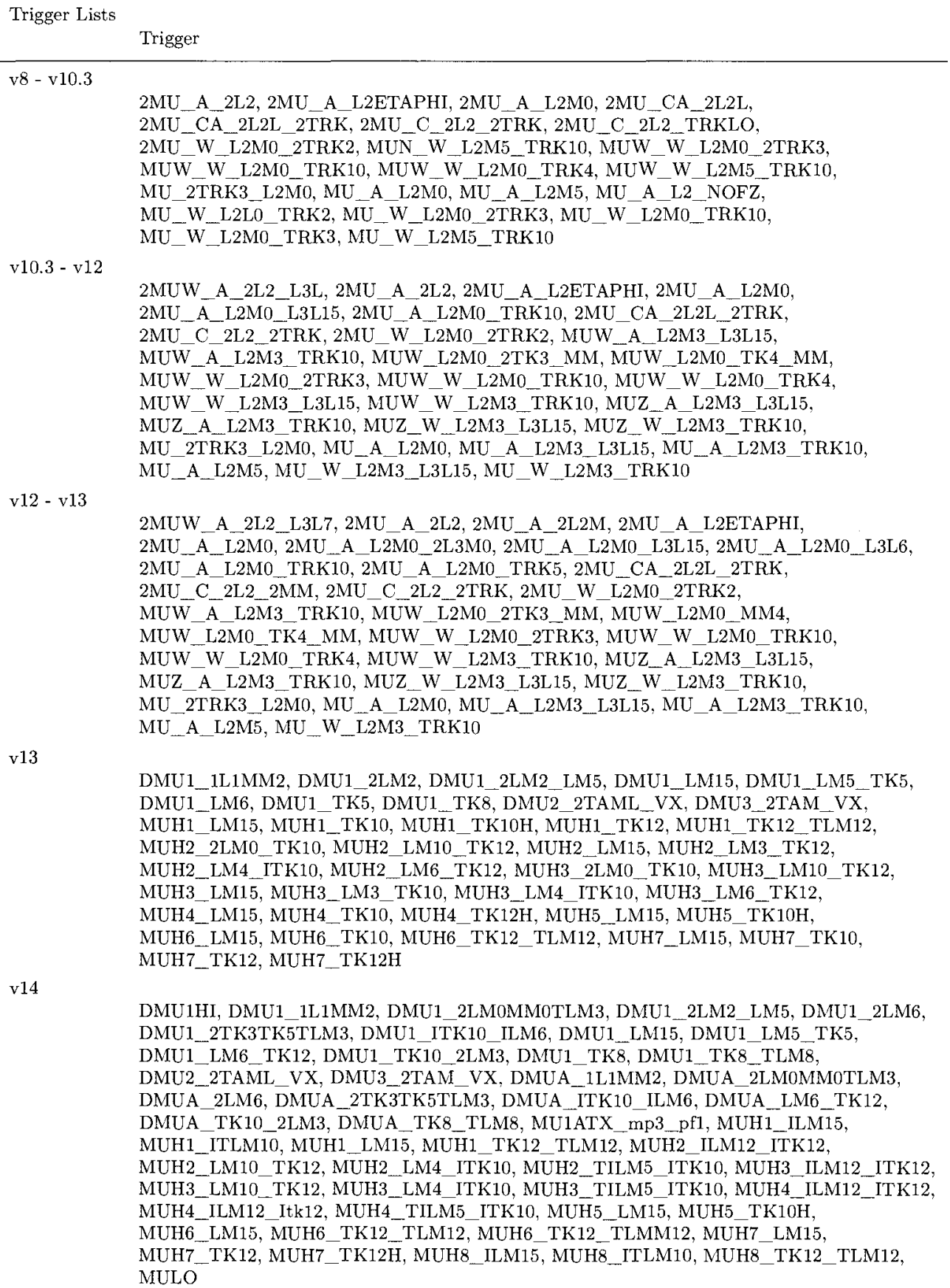

Table B.1: Triggers used per triggerlist period. 


\section{Bibliography}

[1] Weinberg, S. The Quantum Theory of Fields, vol. 1 (Cambridge University Press, 1995). $2,2.1 .3$

[2] Georgi, H. \& Glashow, S. Unity of All Elementary-Particle Forces. Phys. Rev. Lett. 32, 438 (1974). 2.1, 2.3.2

[3] Weinberg, S. A Model of Leptons. Phys. Rev. Lett. 19, 1264-1266 (1967). 2.1, 2.1.2

[4] Goldstone, J., Salam, A. \& Weinberg, S. Broken Symmetries. Phys. Rev. 127, 965-970 (1962). 2.1, 2.1.2

[5] 't Hooft, G. The Conceptual Basis of Quantum Field Theory (2007). URL http://www .phys.uu.nl/ thooft/lectures/basisqft.pdf. 2.1.1

[6] Nambu, Y. \& Jona-Lasinio, G. Dynamical Model of Elementary Particles Based on an Analogy with Superconductivity. I. Phys. Rev. 122, 345-358 (1961). 2.1.2

[7] Anderson, P. W. Plasmons, Gauge Invariance, and Mass. Phys. Rev. 130, 439442 (1963). 2.1.2

[8] Higgs, P. W. Spontaneous Symmetry Breakdown without Massless Bosons. Phys. Rev. 145, 1156-1163 (1966). 2.1.2

[9] Kibble, T. W. B. Symmetry Breaking in Non-Abelian Gauge Theories. Phys. Rev. 155, 1554-1561 (1967). 2.1.2

[10] Englert, F. \& Brout, R. Broken Symmetry and the Mass of Gauge Vector Mesons. Phys. Rev. Lett. 13, 321-323 (1964). 2.1.2

[11] Higgs, P. W. Broken Symmetries and the Masses of Gauge Bosons. Phys. Rev. Lett. 13, 508-509 (1964). 2.1.2

[12] Guralnik, G. S., Hagen, C. R. \& Kibble, T. W. B. Global Conservation Laws and Massless Particles. Phys. Rev. Lett. 13, 585-587 (1964). 2.1.2

[13] Llewellyn-Smith, C. Phys. Lett. B46, 233 (1973). 2.1.3

[14] Cornwall, J., D.Levin \& Tiktopoulos, G. Phys. Rev. Lett. 30, 1268 (1973). 2.1.3 
[15] 't Hooft, G. Renormalization without infinities. In Proc. Coral Gables Conference (2004). http://www.arxiv.org/abs/hep-th/0405032. 2.1.3

[16] Yao, W.-M. et al. Review of Particle Physics. Journal of Physics G 33, 1+ (2006). URL http://pdg. Ibl.gov. 2.2, 2.3.2, 2.3.3, 2.3, 3.10

[17] Drell, S. D. \& Yan, T.-M. Massive lepton-pair production in hadron-hadron collisions at high energies. Phys. Rev. Lett. 25, 316-320 (1970). 2.2

[18] Ellis, R. K., Stirling, W. J. \& Webber, B. R. QCD and Collider Physics (Cambridge University Press, 2003). 2.2, 2.2.1

[19] Abbott, B. et al. Measurement of the High-Mass Drell-Yan Cross Section and Limits on Quark-Electron Compositeness Scales. Physical Review Letters 82, 4769 (1999). URL http://www.arxiv.org/abs/hep-ex/9812010. 2.2

[20] Abe, F. et al. Measurement of $Z^{0}$ and Drell-Yan production cross sections using dimuons in $p \bar{p}$ collisions at $\sqrt{s}=1.8 \mathrm{TeV}$. Phys. Rev. D 59, 052002 (1999). 2.3

[21] Stirling, W. J. \& Whalley, M. R. A compilation of Drell-Yan cross sections. Journal of Physics G: Nuclear and Particle Physics 19, D1-D102 (1993). URL http://stacks.iop.org/0954-3899/19/D1. 2.2 .2

[22] Susskind, L. The gauge hierarchy problem, technicolor, supersymmetry, and all that (talk). Phys. Rept. 104, 181-193 (1984). 2.3.1

[23] 't Hooft, G. Naturalness, chiral symmetry, and spontaneous chiral symmetry breaking. In $\mathrm{H}$. Araki et al. (ed.) Advanced Series in Mathematical Physics, vol. 19 (World Scientific, Singapore, 1994). 2.3.1, 2.3.1

[24] de Boer, W. Grand unified theories and supersymmetry in particle physics and cosmology. Prog. Part. Nucl. Phys. 33, 201-302 (1994). http://www.arxiv. org/abs/hep-ph/9402266. 2.4

[25] Baez, J. C. Calabi-yau manifolds and the standard model (2005). URL http: //www. citebase.org/abstract?id=oai : arXiv.org:hep-th/0511086. 2.3.2

[26] Langacker, P. Grand Unified Theories and Proton Decay. Phys. Rept. 72, 185 (1981). 2.3.2

[27] Babu, K. S., Kolda, C. \& March-Russell, J. Implications of generalized Z-Z' mixing. Phys. Rev. D 57, 6788-6792 (1998). http://www.arxiv.org/abs/hep-ph/ 9710441. 2.3.3

[28] Carena, M. S., Daleo, A., Dobrescu, B. A. \& Tait, T. M. P. Z' gauge bosons at the Tevatron. Phys. Rev. D 70, 093009 (2004). http://www.arxiv.org/abs/ hep-ph/0408098. 2.3.3,6.2.4, 2, A

[29] Appelquist, T., Cheng, H.-C. \& Dobrescu, B. A. Bounds on universal extra dimensions. Physical Review D 64, 035002 (2001). URL http://www.arxiv. org/abs/hep-ph/0012100. 8 
[30] Abdallah, J. et al. Measurement and interpretation of fermion-pair production at LEP energies above the Z resonance. Eur. Phys. J. C45, $589-632$ (2006). http://www.arxiv.org/abs/hep-ex/0512012. 2.3 .3

[31] Abbiendi, G. et al. Tests of the standard model and constraints on new physics from measurements of fermion-pair production at $189-\mathrm{GeV}$ to $209-\mathrm{GeV}$ at LEP. Eur. Phys. J. C33, 173-212 (2004). http://www.arxiv.org/abs/hep-ex/ 0309053. 2.3 .3

[32] Abulencia, A. et al. Search for $Z^{\prime} \rightarrow e^{+} e^{-}$using dielectron mass and angular distribution. Phys. Rev. Lett. 96, 211801 (2006). http://www.arxiv.org/abs/ hep-ex/0602045. 2.3.3, 7

[33] D $\emptyset$ Collaboration. Search for Heavy Z Bosons in the Dielectron Channel with $200 \mathrm{pb}^{-1}$ of Data with the $\mathrm{D} \emptyset$ Detector. In DØnote 4375-Conf (2004). 2.3.3, 7

[34] D $\emptyset$ Collaboration. Search for Heavy Z Bosons in the Dimuon Channel with 250 $\mathrm{pb}^{-1}$ of Data with the D $\emptyset$ Detector. In D $\emptyset$ note $4577-$ Conf (2004). 2.3.3

[35] Cheung, K.-M. Constraints on electron quark contact interactions and implications to models of leptoquarks and extra Z bosons. Phys. Lett. B517, 167-176 (2001). http://www .arxiv.org/abs/hep-ph/0106251. 2.3.3

[36] Arkani-Hamed, N., Cohen, A. G. \& Georgi, H. Electroweak symmetry breaking from dimensional deconstruction. Physics Letters B 513, 232 (2001). URL http://www. arxiv.org/abs/hep-ph/0105239. 2.3.4

[37] Arkani-Hamed, N., Cohen, A. G., Katz, E. \& Nelson, A. E. The littlest higgs. JHEP 0207, 034 (2002). URL http://www.arxiv.org/abs/hep-ph/0206021. 2.3 .4

[38] Han, T., Logan, H. E., McElrath, B. \& Wang, L.-T. Phenomenology of the little higgs model. Physical Review D 67, 095004 (2003). URL http://arxiv.org/ abs/hep-ph/0301040. 2.3.4, 2.5

[39] Arkani-Hamed, N., Dimopoulos, S. \& Dvali, G. The Hierarchy Problem and New Dimensions at a Millimeter. Physics Letters B 429, 263 (1998). URL http://www.arxiv.org/abs/hep-ph/9803315. 2.3.5

[40] Arkani-Hamed, N., Dimopoulos, S. \& March-Russell, J. Stabilization of SubMillimeter Dimensions: The New Guise of the Hierarchy Problem. Physical Review D 63, 064020 (2001). URL http://www.citebase.org/abstract?id= oai : arXiv .org: hep-th/9809124. 2.3.5

[41] Arkani-Hamed, N., Dimopoulos, S. \& Dvali, G. Phenomenology, astrophysics and cosmology of theories with sub-millimeter dimensions and tev scale quantum gravity. Physical Review D 59, 086004 (1999). URL http://www.citebase.org/ abstract?id=oai: arXiv. org:hep-ph/9807344. 11 
[42] Adelberger, E. G., Heckel, B. R. \& Nelson, A. E. Tests of the gravitational inverse-square law. Annual Review of Nuclear and Particle Science 53, 77 (2003). URL http://www. citebase.org/abstract? id=oai : arXiv. org: hep-ph/0307284. 2.3.5

[43] Randall, L. \& Sundrum, R. A Large Mass Hierarchy from a Small Extra Dimension. Phys. Rev. Lett. 83, 3370 (1999). URL http://arxiv.org/abs/hep-ph/ 9905221. 2.3.5

[44] Rizzo, T. G. Pedagogical Introduction to Extra Dimensions (2004). URL http: //arxiv.org/abs/hep-ph/0409309. 2.3.5

[45] Davoudiasl, H., Hewett, J. L. \& Rizzo, T. G. Phenomenology of the RandallSundrum Gauge Hierarchy Model. Phys. Rev. Lett. 84, 2080-2083 (2000). 2.3.5

[46] Davoudiasl, H., Hewett, J. L. \& Rizzo, T. G. Experimental probes of localized gravity: On and off the wall. Phys. Rev. D 63, 075004 (2001). 2.3.5

[47] Pomarol, A. Gauge bosons in a five-dimensional theory with localized gravity. Physics Letters B 486, 153-157 (2000). 2.3.5

[48] Abazov, V. M. et al. Search for Randall-Sundrum gravitons with $1 \mathrm{fb}^{-1}$ of data from ppbar collisions at sqrt(s) $=1.96 \mathrm{TeV}$. Phys. Rev. Lett. 100, 091802 (2008). $0710.3338 .2 .3 .5,7$

[49] Fermilab Beams division. Collider Run II Peak Luminosity. URL http: //www-bdnew.fnal.gov/operations/lum/lum.html. 3.1, 3.2

[50] Cole, T. et al. A Report on the Design of the Fermi National Accelerator Laboratory Superconducting Accelerator. Beams-doc-1888-v1 (1979). 3.1

[51] Schmidt, C. W. Review of negative hydrogen ion sources Presented at 1990 Linear Accelerator Conf., Albuquerque, NM, Sep 10-14, 1990. 3.1.1

[52] Cockcroft, J. D. \& Walton, E. T. S. Experiments with High Velocity Positive Ions. Royal Society of London Proceedings Series A 136, 619-630 (1932). 3.1.1

[53] Fermilab Beams division. Linac Rookie Book. URL http://www-bdnew.fnal. gov/operations/rookie_books/LINAC_v2.pdf. 3.1.1

[54] Fermilab Beams division. Booster Rookie Book. URL http://www-bdnew fnal. gov/operations/rookie_books/Booster_V3_1.pdf. 3.1.1

[55] Hojvat, C. et al. The Multiturn Charge Exchange Injection System for the Fermilab Booster Accelerator FERMILAB-TM-0872. 3.1.1

[56] Fermilab Beams division. Main Injecter Rookie Book. URL http://www-bdnew . fnal.gov/operations/rookie_books/Main_Injector_v1.pdf. 3.1.1

[57] Fermilab Beams division. Antiproton Source Rookie Book. URL http:// www-bdnew. fnal.gov/operations/rookie_book/Pbar_V1_1.pdf. 3.1.2 
[58] Fermilab Beams division. Concepts Rookie Book. URL http://www-bdnew . fnal.gov/operations/rookie_books/Concepts_V3.pdf. 3.1.2

[59] Abazov, V. M. et al. The upgraded D $\emptyset$ detector. Nucl. Instrum. Meth. A565, 463-537 (2006). http://www.arxiv.org/abs/physics/0507191. 3.2, 3.11

[60] Miao, C.-C. The D $\emptyset$ Run II luminosity monitor. Nucl. Phys. Proc. Suppl. 78, $342-347$ (1999). 3.2.2

[61] Casey, B. et al. Determination of the effective inelastic $p \bar{p}$ cross-section for the $\mathrm{d} \emptyset$ luminosity measurement using upgraded readout electronics (2005). D $\emptyset$ internal note 4958. 3.2.2

[62] Klimenko, S., Konigsberg, J. \& Liss, T. M. Averaging of the inelastic cross sections measured by the CDF and the E811 experiments FERMILAB-FN-0741. 3.2 .2

[63] Weber, M. S. The D $\emptyset$ silicon micro-strip tracker. Nucl. Instrum. Meth. A560, 14-17 (2006). 3.2.3

[64] Adams, D. et al. The D $\emptyset$ Upgrade: Central Fiber Tracker, Technical Design Report. DØ Note 4164 (2003). 3.2.3

[65] Brzezniak, J. et al. Conceptual design of a 2-Tesla superconducting solenoid for the Fermilab D $\emptyset$ detector upgrade FERMILAB-TM-1886. 3.2.3

[66] Groer, L. D $\emptyset$ calorimeter upgrades for Tevatron Run II. Frascati Phys. Ser. 21, $377-394$ (2001). 3.2.4

[67] Leo, W. R. Techniques in nuclear and particle physics experiments: a how-to approach Berlin, Germany: Springer (1987) 368 p. 3.2.4

[68] Fabjan, C. W. \& Gianotti, F. Calorimetry for particle physics. Rev. Mod. Phys. 75, $1243-1286$ (2003). 3.2.4

[69] Abazov, V. M. et al. The muon system of the Run II D $\emptyset$ detector. Nucl. Instrum. Meth. A552, 372-398 (2005). http://www.arxiv.org/abs/physics/0503151. 3.2 .5

[70] Askew, A. Parametrizing the local muon resolution in p13 data for pmes (2003). $\mathrm{D} \emptyset$ internal note $4314 \cdot 3.2 .5$

[71] Sjostrand, T., Lonnblad, L., Mrenna, S. \& Skands, P. Pythia 6.3 Physics and Manual. 4, 4.1.2

[72] Stump, D. et al. Inclusive jet production, parton distributions, and the search for new physics. Journal of High Energy Physics 2003, 046 (2003). URL http: //stacks . iop.org/1126-6708/2003/i=10/a=046. 4, 4.2,6.2.1

[73] Pumplin, J. et al. New generation of parton distributions with uncertainties from global qed analysis. JHEP 0207, 012 (2002). URL http://arxiv.org/ abs/hep-ph/0201195. 4 
[74] Fisyak, Y. \& Womersley, J. D $\emptyset$ gstar D $\emptyset$ GEANT simulation of the total apparatus response (1997). DØ internal note 3191. 4

[75] Brun, R. \& Carminati, F. GEANT detector description and simulation tool. CERN Program Library Long Writeup W5013 (1993). 4

[76] DØsim. URL http://www-d0.fnal.gov/computing/MonteCarlo/simulation/ dosim.html. 4

[77] Hamberg, R., van Neerven, W. L. \& Matsuura, T. A Complete calculation of the order alpha-s**2 correction to the Drell-Yan K factor. Nucl. Phys. B359, $343-405$ (1991). 4.2

[78] Nunnemann, T. NNLO Cross-Sections for Drell-Yan, $Z$ and $W$ Production using Modern Parton Distribution Functions (2004). D $\emptyset$ internal note 4476. 4.2, 5.4.1, 6.2 .3

[79] Calame, C. M. C., Montagna, G., Nicrosini, O. \& Vicini, A. Precision electroweak calculation of the production of a high transverse-momentum lepton pair at hadron colliders (2007). URL http://www. citebase.org/abstract?id=oai : arXiv.org:0710.1722. 4.2

[80] Tiller, B. \& Nunnemann, T. Measurement of the differential $Z^{0}$-boson production cross-section as function of transverse momentum (2004). D $\emptyset$ internal note 4660 . $4.5,4.2,4.2$

[81] $\mathrm{D} \emptyset$ Collaboration. Measurement of the inclusive differential cross section for $\mathrm{Z}$ bosons as a function of transverse momentum in $\bar{p} p$ collisions at $\sqrt{s}=1.8 \mathrm{tev}$. Phys. Rev. D 61 (1997). 4.2

[82] Calfayan, P. et al. Muon Identification Certification for p17 data (2006). D $\emptyset$ internal Note 5157. 5.2, 5.2.1, 5.2.2, 5.3, 5.4.3

[83] Abdallah, T. D. C. J. b-tagging in DELPHI at LEP. European Physical Journal C 32, 185 (2004). URL http://www.arxiv.org/abs/hep-ex/0311003. 5.2.3

[84] Paterno, M. Calculating efficiencies and their uncertainties URL http://home. fnal.gov/ paterno/images/effic.pdf. FERMILAB-TM-2286-CD. 2

[85] Blazey, G. C. et al. Run II jet physics (2000). hep-ex/0005012. 3

[86] Punzi, G. Sensitivity of searches for new signals and its optimization (2007). Talk from PhyStat2003, Stanford, Ca, USA, September 2003, PSN MODT002., http://www . arxiv.org/abs/physics/0308063. 6.1, 6.1.1

[87] Rizzo, T. G. Z' phenomenology and the LHC (2006). hep-ph/0610104. 7

[88] Bayatian, G. L. et al. CMS technical design report, volume II: Physics performance. J. Phys. G34, 995-1579 (2007). 7 


\section{Samenvatting}

Voor het onderzoek dat hier gepresenteerd is, werden $1 \mathrm{fb}^{-1}$ van geselecteerde hoge transverse momentum dimuon data bekeken, welke verzameld waren met de $\mathrm{D} \varnothing$ detector. Deze waren verkregen uit proton-antiproton botsingen in de Tevatron versneller te Fermilab bij een energie van $\sqrt{s}=1.96 \mathrm{TeV}$.

In deze data werd gezocht naar resonanties bovenop het Drell-Yan spectrum met een invariante massa veel hoger dan de $Z$ boson massa. Zulke resonanties worden voorspeld door verschillende modellen van nieuwe fysica; in dit werk werden de bevindingen geïnterpreteerd voor Standaard Model-achtige $Z^{\prime}$ modellen, generieke U(1) extensies van het Standaard Model, "little Higgs" modellen en het gekromde extra dimensie model van Randall en Sundrum.

Door de schone eigenschappen van de dimuon eindtoestand kon de reduceerbare achtergrond grotendeels verwijderd worden door een combinatie van snedes van welke de efficientie bepaald werd op de $Z \rightarrow \mu^{+} \mu^{-}$piek, waarvan de werkzame doorsnede en massa bekend zijn. Om de achtergrond van $Z \rightarrow \mu^{+} \mu^{-}$gebeurtenissen met een veel te hoog gereconstrueerde dimuon massa te reduceren werd een kinematische fit uitgevoerd, gebruikmakende van het gegeven dat de totale transverse energie in een botsing op moet tellen tot nul.

Het gevonden massa spectrum werd vervolgens vergeleken met de verwachte verdeling, met inachtneming van de systematische onzekerheden op de Standaard Model werkzame doorsnede, de detector efficienties en de transverse momentum resolutie. Hierbij werd voor elk model en $Z^{\prime}$ massa onder beschouwing een eindselectie op de invariante dimuon massa gemaakt die de gevoeligheid van een tel experiment voor de aanwezigheid van een signaal maximaliseert.

Aangezien er geen significant overschot op de voorspelling werd gevonden, zijn er bovenlimieten gesteld op de productie van een hypothetisch nieuw boson maal de vervalbreedte naar dimuonen voor elk van de modellen, uitgezet tegen de massa van het hypothetische nieuwe deeltje. Voor een Standaard Model-achtige $Z^{\prime}$ werd een onderlimiet op de massa gevonden van $M_{Z^{\prime}}>838 \mathrm{GeV} / c^{2}$; voor het Randall-Sundrum model werd een onderlimiet gevonden van $M_{G^{[1]}}>693 \mathrm{GeV} / c^{2}$ voor $k / \bar{M}_{\mathrm{Pl}}=0.1$. Hiermee werden de tot op heden meest beperkende limieten op $\sigma\left(p \bar{p} \rightarrow Z^{\prime}+X\right) \times$ $\mathrm{BR}\left(Z^{\prime} \rightarrow \mu^{+} \mu^{-}\right)$en $\sigma\left(p \bar{p} \rightarrow G^{[1]}+X\right) \times \mathrm{BR}\left(G^{[1]} \rightarrow \mu^{+} \mu^{-}\right)$gesteld. 


\section{Acknowledgements}

Many people have been instrumental for the completion of the book you have in your hands; some academically, some by their support and example. My gratitude goes to all of them.

I wish to thank my supervisors Stan Bentvelsen and Paul de Jong for giving the opportunity to make the attempt in the first place and for their patience in helping me finish it. Without their diligence, this thesis would have taken even longer to complete.

In Illionois, at fermilab I had a fantastic time, first of all with Pieter, Miruna and Jeroen. We all made it and no one got killed. Not even trying to get from Vancouver to Chicago non-stop.

I also must thank the girls and the whole $24 / 7 \mathrm{bbq}$ slash party experience that they had going on there. Ana, Helena and Renata, I don't quite know how you managed, hats off! I have many good memories of hanging out at the Brazilian house, cruising downtown and travelling around the country. A big salute also to all the other crazy physicists, Alejo, Charlie, Mario, Ingo, Santiago, Cristina, Kirby and of course "wazzup" Carsten. Cheers! And Arnd, thank you for sharing many beers and wise words about my research.

Back home, I had old friends who had the courtesy never to ask me what substances I needed to inject in order to produce such a volume of gibberish. Robert, Vincent, Rogier, Derk, Tatjana and many others, you have been great support for me through the hard times.

The friends and colleagues in Amsterdam, Niels, Erik, Bram, Martijn, "big" Jeroen, Gordon, Joana (job!), Ronald (house!), Claudine, Eleonora, Ermes and many others, I found in you a willing ear for complaints, advice and shared experiences but mostly excuses to sport, party, have too much coffee or drinks on friday at 4.

I also want to thank my family: my parents, my sister, my grandmother and grandfather (who had a surprisingly good understanding anything of my research!) and all others. It was comforting to know there there were some people who never had doubts that I would bring this to a completion.

And in the end, most of all I want to thank Ana, my beautiful wife-to-be, for all the support and love she gives me. Ana, meeting you there made it all worth it. During the struggle that led to this book, we started dating, hooked up, survived Fermilab, Alaska and living on a different continent, and finally moved in together. Beijos! 
